\title{
Anmeldung und Feststellung von Forderungen
}

\section{im Insolvenzverfahren}

\author{
Dissertation \\ zur Erlangung des Doktorgrades \\ der Juristischen Fakultät \\ der Georg-August-Universität zu Göttingen \\ vorgelegt \\ von
}

Mark A. Jaeschke

aus Hannover

Göttingen 2011 
Berichterstatter: Professor Dr. Martin Ahrens

Mitberichterstatter: Professor Dr. Volker Lipp

Tag der mündlichen Prüfung: 13.04.2011 
Meiner Familie 


\section{Inhaltsübersicht}

Inhaltsverzeichnis

II

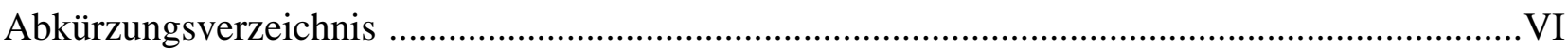

Literaturverzeichnis

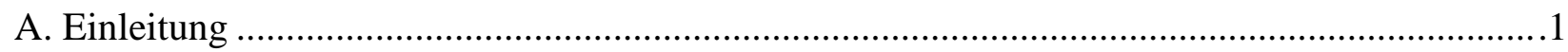

B. Stellung und Funktion des Anmelde- und Feststellungsverfahrens ...................................... 4

C. Teilnahmevoraussetzungen für das Verfahren ..................................................................10

I. Die Insolvenzforderung und ihr Gläubiger i.S.d. § 174 I 1 InsO ....................................10

II. Voraussetzungen für eine Qualifikation als Insolvenzforderung ....................................16

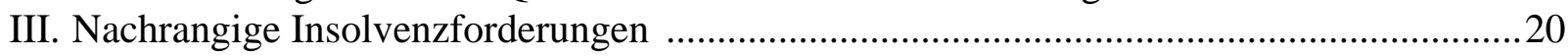

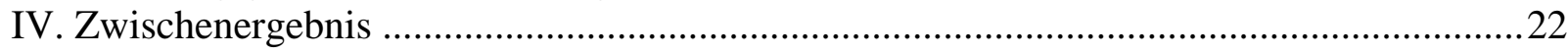

D. Anmeldung und Tabelleneintragung der Insolvenzforderungen ..........................................23

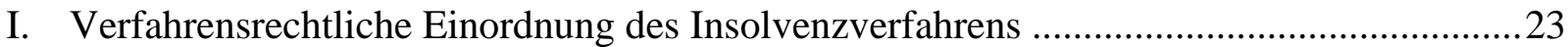

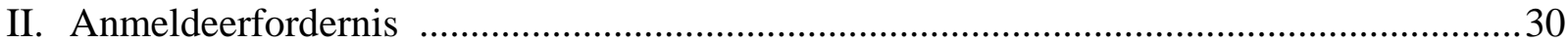

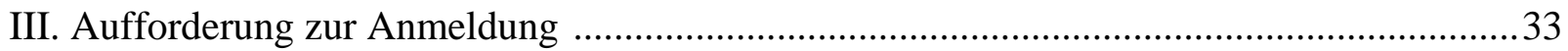

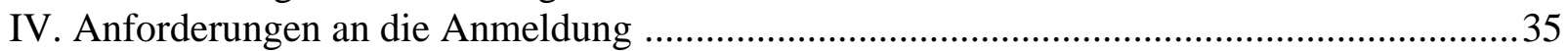

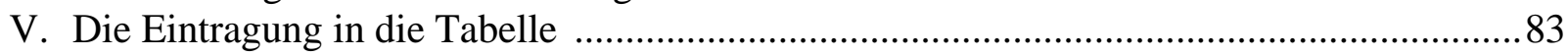

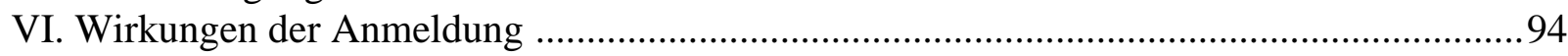

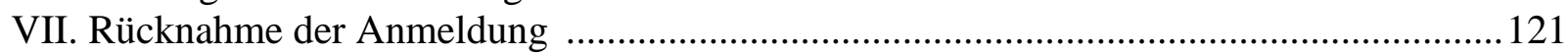

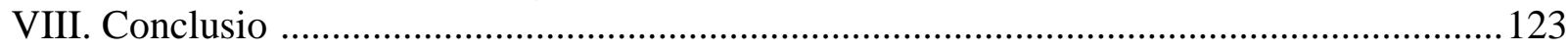

E. Prüfung und Feststellung der Insolvenzforderungen ........................................................127

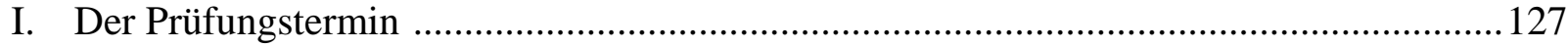

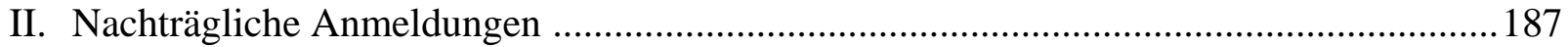

III. Die Feststellung der Insolvenzforderungen im Prüfungstermin ....................................206

IV. Wirkung der Nicht-/Feststellung auf die Rechte des Anmeldenden ...............................235

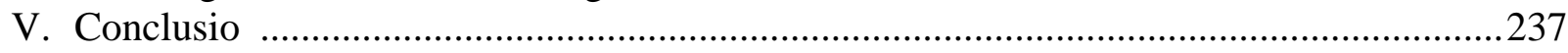

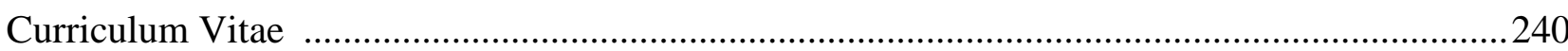




\section{Inhaltsverzeichnis}

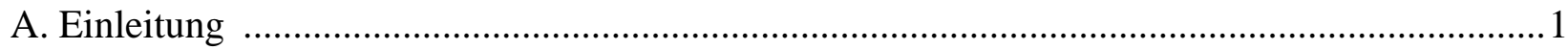

B. Stellung und Funktion des Anmelde- und Feststellungsverfahrens ......................................4

C. Teilnahmevoraussetzungen für das Verfahren .............................................................. 10

I. Die Insolvenzforderung und ihr Gläubiger i.S.d. § 174 I 1 InsO .......................................10

II. Voraussetzungen für eine Qualifikation als Insolvenzforderung ......................................16

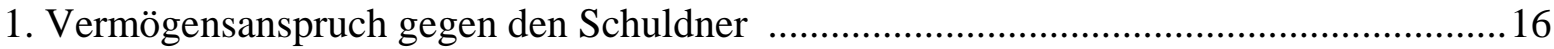

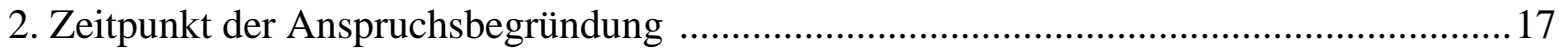

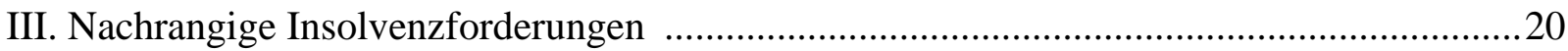

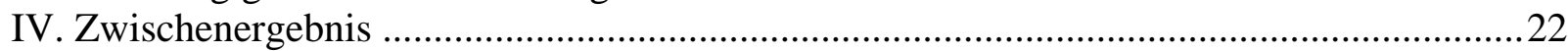

D. Anmeldung und Tabelleneintragung der Insolvenzforderungen ..........................................23

I. Verfahrensrechtliche Einordnung des Insolvenzverfahrens .............................................23

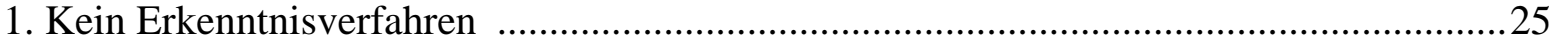

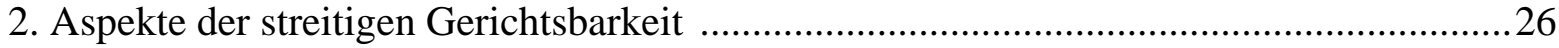

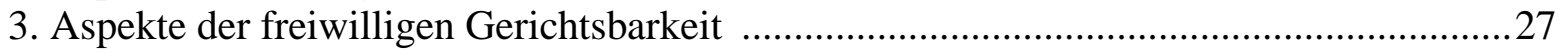

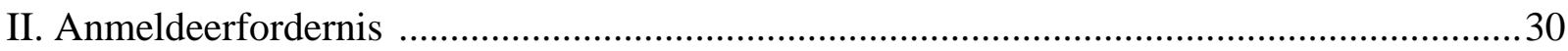

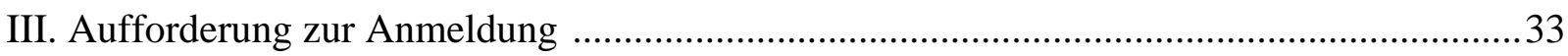

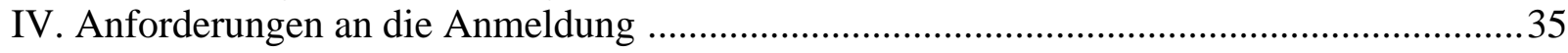

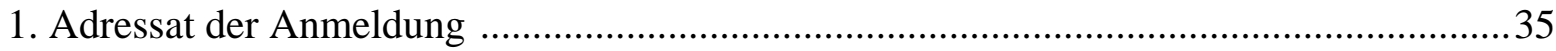

2. Die wesentliche Form der Forderungsanmeldung .........................................................36

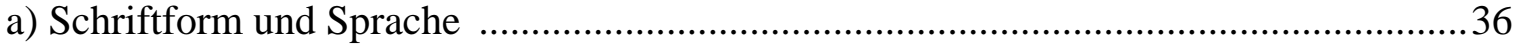

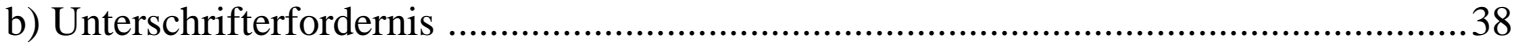

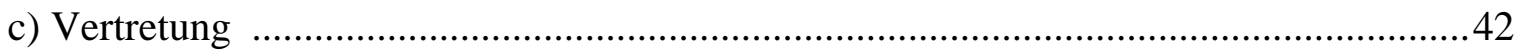

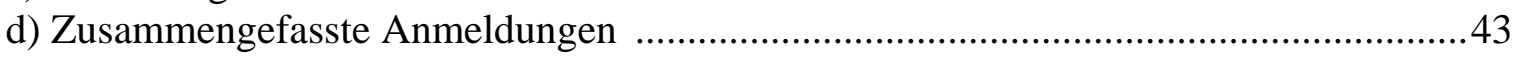

3. Der wesentliche Inhalt der Forderungsanmeldung .....................................................4

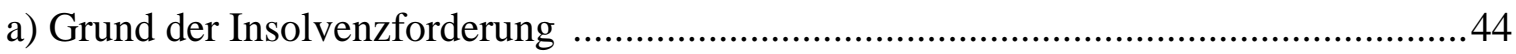

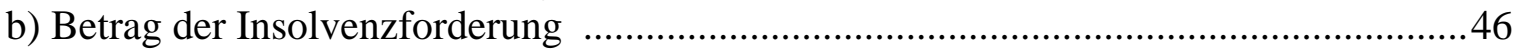

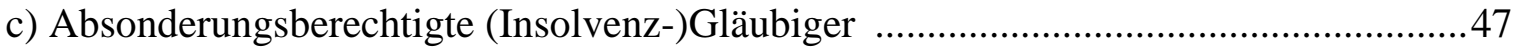

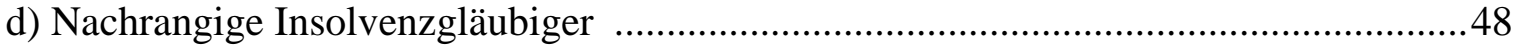

e) Insolvenzgläubiger aus anderen EU-Mitgliedstaaten .................................................48 
4. Beizufügende Urkunden und sonstige Beweisstücke .49

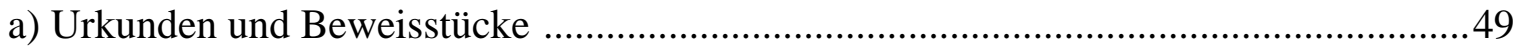

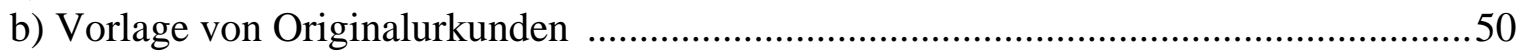

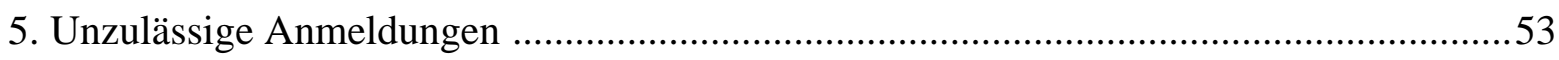

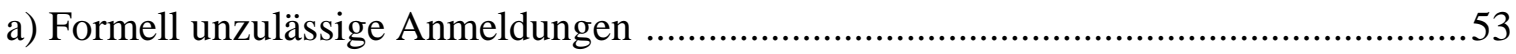

b) Differenzierung formelle - materielle Prüfung der Anmeldung ...................................56

c) Prüfung durch Urkundsbeamte unter der Konkursordnung ........................................61

d) Prüfung durch Insolvenzverwalter unter der Insolvenzordnung ..................................65

e) Rechtsbehelfe gegen die Zurückweisung des Insolvenzverwalters .............................67

aa) Einschreiten des Insolvenzgerichts im Aufsichtswege .......................................68

bb) Prüfung durch das Insolvenzgericht im Prüfungstermin ....................................69

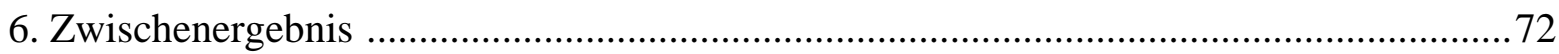

7. Fehler und Ergänzung/Änderung der zulässigen Anmeldung ........................................72

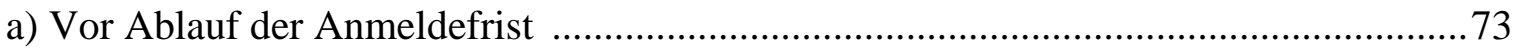

b) Nach Ablauf der Anmeldefrist bis zur Feststellung der Forderung ..............................73

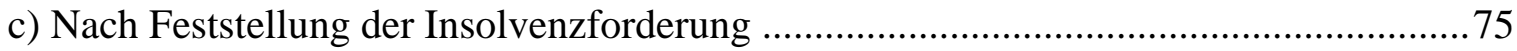

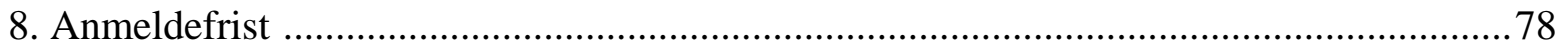

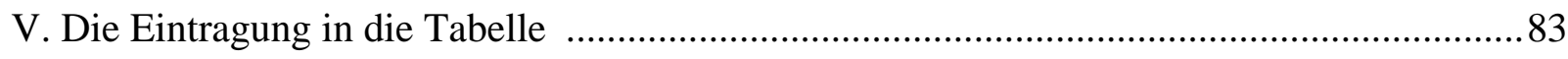

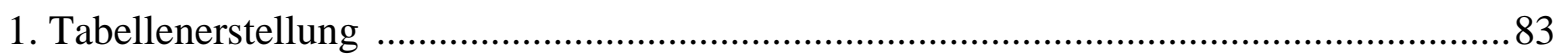

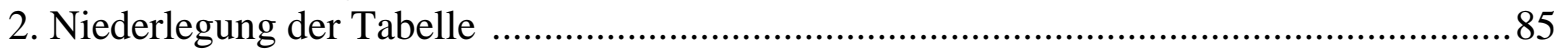

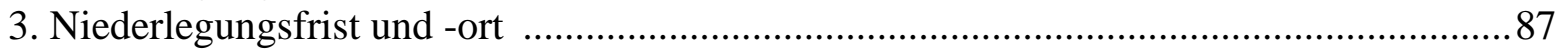

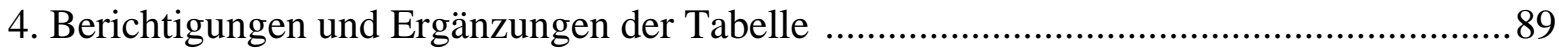

5. Hinweispflicht des Insolvenzgerichts bei Deliktsforderungen .......................................90

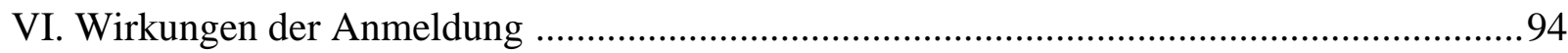

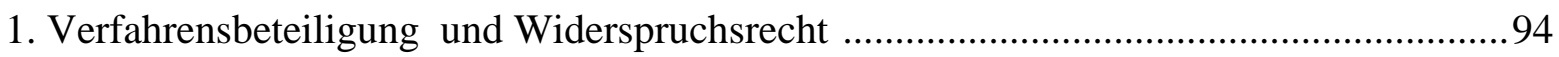

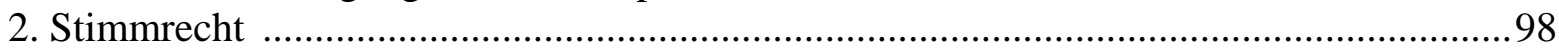

a) Stimmrechtsgewährung und Einigung über das Stimmrecht .....................................99

b) Stimmrecht bei Widerspruch des Schuldners in Eigenverwaltung ..............................102

c) Stimmrechtsfestsetzung durch das Insolvenzgericht ................................................ 105

3. Akteneinsicht und Erteilung von Abschriften/Kopien .............................................108

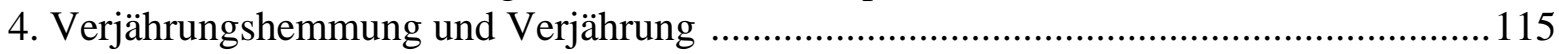

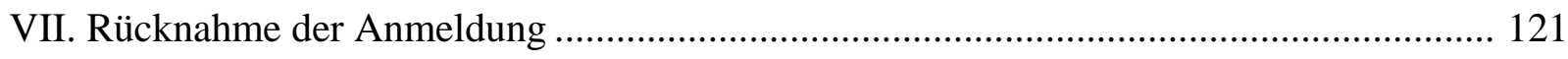

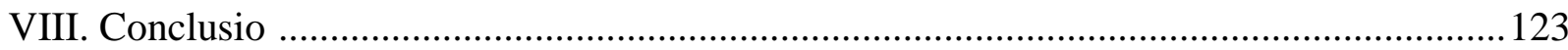


E. Prüfung und Feststellung der Insolvenzforderungen

I. Der Prüfungstermin.

1. Prüfungstermin - Berichtstermin 128

a) Stimmrecht angemeldeter ungeprüfter Insolvenzforderungen .....................................129

b) Stimmrecht bisher unangemeldeter Insolvenzforderungen .........................................131

2. Teilnahme am Prüfungstermin .....................................................................................134

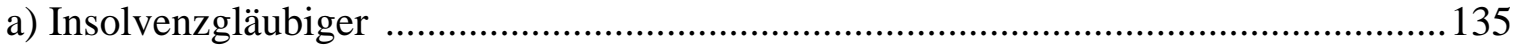

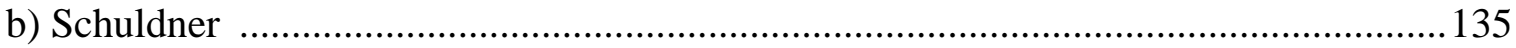

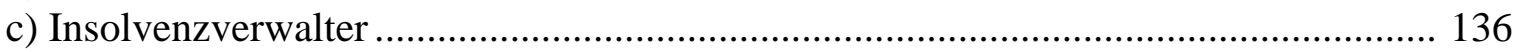

3. Leitung und Durchführung des Prüfungstermins ......................................................... 137

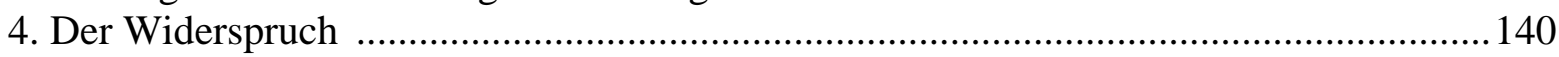

a) Widerspruchsberechtigung und Widerspruchspflicht ..................................................141

b) Widerspruchszeitpunkt ..................................................................................... 142

c) Form und Inhalt des Widerspruchs ........................................................................... 144

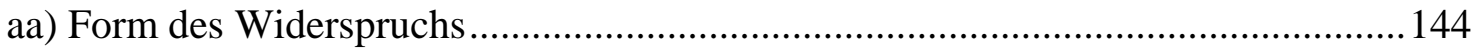

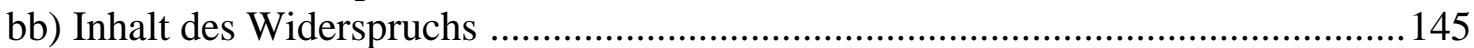

сc) Isolierte Widerspruchsmöglichkeit gegen das Forderungsattribut ..........................148

d) Wirkung des Widerspruchs ......................................................................................156

aa) Eigenverwaltung des Schuldners ……………………......................................157

bb) Das „vorläufige Bestreiten“ des Insolvenzverwalters ............................................159

e) Verlust des Widerspruchsrechts...............................................................................163

aa) Bindung an bestrittene Insolvenzforderung .........................................................163

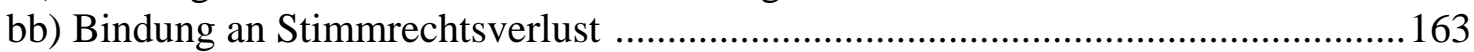

cc) Bindung an die formelle Insolvenzgläubigerstellung........................................... 165

f) Rücknahme und Beseitigung des Widerspruchs ........................................................167

aa) Rücknahme des Widerspruchs ............................................................................168

bb) Beseitigung des Widerspruchs und Betreibungslasten ........................................170

(1) Widerspruch durch den Insolvenzgläubiger/-verwalter ....................................170

(2) Widerspruch durch den Schuldner ..................................................................175

II. Nachträgliche Anmeldungen ........................................................................................ 187

1. Anmeldung und Eintragung verspäteter Insolvenzforderungen .......................................188

2. Insolvenzforderungsanmeldung bis zum Ende des Prüfungstermins ...............................189 
3. Insolvenzforderungsanmeldung nach dem Prüfungstermin 192

a) Letzte Anmelde- und Prüfungsmöglichkeit ............................................................... 192

b) Aufnahme der Insolvenzforderung ins Schlussverzeichnis .....................................194

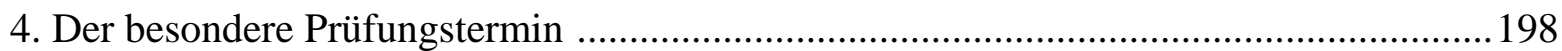

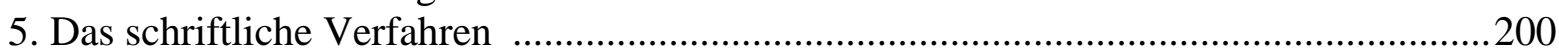

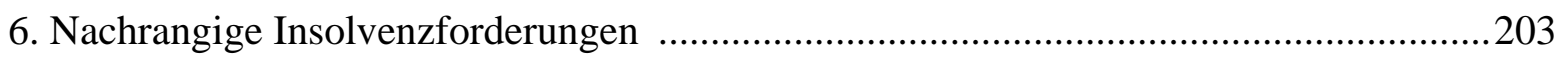

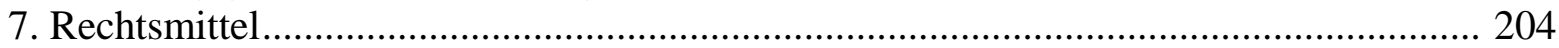

III. Die Feststellung der Insolvenzforderungen im Prüfungstermin ...................................206

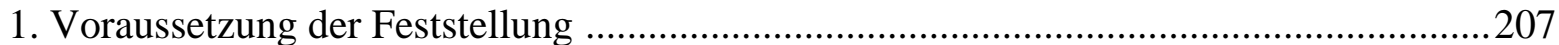

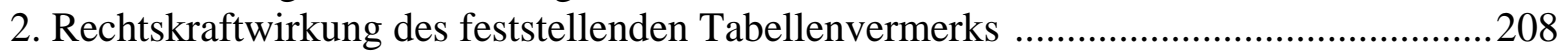

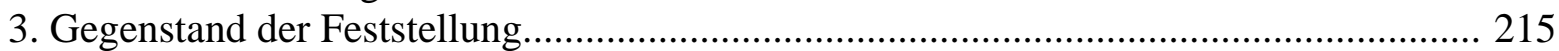

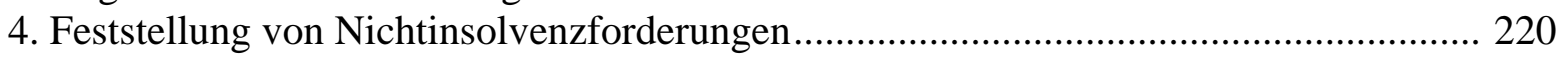

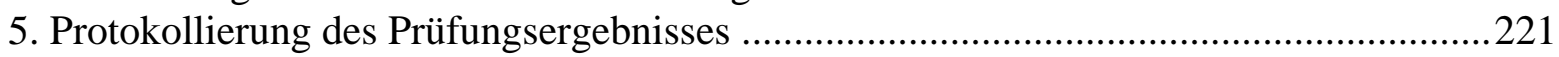

a) Vorabeintragung des Prüfungsergebnisses und Unterschrift(en)............................. 222

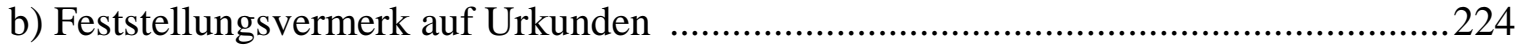

c) Ursprünglicher Titel - vollstreckbarer Tabellenauszug .............................................225

6. Berichtigung unrichtiger oder fehlender Eintragung in die Tabelle ..............................231

7. Rechtsbehelf gegen die festgestellte Insolvenzforderung .........................................234

IV. Wirkung der Nicht-/Feststellung auf die Rechte des Anmeldenden ................................235

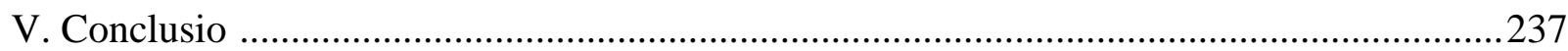




\section{Abkürzungsverzeichnis}

a.A.

Abs.

a.F.

AG

AktG

Alt.

Anm.

AnwBl.

$\operatorname{Art}(\mathrm{t})$.

BAG

BB

Begr.

Beschl.

$\mathrm{BFH}$

BGB

BGBl.

BGH

BGHZ

BR-Drucks.

BT-Drucks.

BVerfG

BVerfGE

bzgl.

bzw.

ders.

DGVZ

d.h.

dies.

Diss.

DZWiR

e.G.

EG

EG-ABl.

EGInsO

EU

EuInsVO

etc.

e.V.

evtl.

EWiR andere Ansicht

Absatz

alte Fassung

Amtsgericht

Aktiengesetz

Alternative

Anmerkung

Anwaltsblatt

Artikel

Bundesarbeitsgericht

Zeitschrift „Der Betriebs-Berater“

Begründung

Beschluss

Bundesfinanzhof

Bürgerliches Gesetzbuch

Bundesblatt der Bundesrepublik Deutschland

Bundesgerichtshof

Entscheidungen des Bundesgerichtshofes in Zivilsachen

Drucksachen des Deutschen Bundesrates

Drucksachen des Deutschen Bundestages

Bundesverfassungsgericht

Entscheidungen des Bundesverfassungsgerichts

bezüglich

beziehungsweise

derselbe

Deutsche Gerichtsvollzieher Zeitung

das heißt

dieselbe(n)

Dissertation

Deutsche Zeitschrift für Wirtschaftsrecht

eingetragene Genossenschaft

Europäische Gemeinschaft

Amtblatt der Europäischen Gemeinschaft

Einführungsgesetz zur Insolvenzordnung

Europäische Union

Europäische Verordnung über Insolvenzverfahren

et cetera

eingetragener Verein

eventuell

Entscheidungen zum Wirtschaftsrecht 
$\begin{array}{ll}\text { f./ ff. } & \text { folgend/e } \\ \text { Fn. } & \text { Fußnote } \\ \text { FS } & \text { Festschrift }\end{array}$

gem.

GesO

ggf.

GKG

$\mathrm{GmbH}$

GmbHG

GmS-OGB

GenG

GVG

Habil.

h.M.

Hrsg.

InsO

InsVV

InVo

i.S.d.

i.V.m.

JKomG

KO

KTS

LG

LSG

MDR

m.w.N

NJW

NZI

OLG

RegE.

RG

RGZ

Rpfleger

RPflG

s.

S.

SGB

gemäß

siehe

Seite
Gesamtvollstreckungsordnung

gegebenenfalls

Gerichtskostengesetz

Gesellschaft mit beschränkter Haftung

Gesetz betreffend die Gesellschaften mit beschränkter Haftung

Gemeinsamer Senat der obersten Gerichte des Bundes

Gesetz betreffend die Gewerbs- und Wirtschaftsgenossenschaften

Gerichtsverfassungsgesetz

Habilitation

herrschende Meinung

Herausgeber

Insolvenzordnung

Insolvenzrechtliche Vergütungsordnung

Insolvenz \& Vollstreckung

im Sinne des/der

in Verbindung mit

Justizkommunikationsgesetzes

Konkursordnung

Zeitschrift „Konkurs-, Treuhand- und Schiedsgerichtswesen“

Landgericht

Landessozialgericht

Monatszeitschrift für Deutsches Recht

mit weiteren Nachweisen

Neue juristische Wochenschrift

Neue Zeitschrift für das Recht der Insolvenz und Sanierung

Oberlandesgericht

Regierungsentwurf

Reichsgericht

Entscheidungen des Reichsgerichts in Zivilsachen

Zeitschrift „Der Deutsche Rechtspfleger“

Rechtspflegergesetz

Sozialgesetzbuch 
SigG

sog.

StGB

u.a.

Univ.

Urt.

v.

VerglO

vgl.

VglO

$\mathrm{VO}$

WM

z.B.

ZIP

ZInsO

$\mathrm{ZPO}$

ZVI

ZZP
Signaturgesetz

so genannte

Strafgesetzbuch

unter anderem/ und andere

Universität

Urteil

vom/von

Vergleichsordnung

vergleiche

Vergleichsordnung

Verordnung

Zeitschrift „Wertpapier-Mitteilungen“

zum Beispiel

Zeitschrift für Wirtschaftsrecht und Insolvenzpraxis

Zeitschrift für das gesamte Insolvenzrecht

Zivilprozessordnung

Zeitschrift für Verbraucher- und Privat-Insolvenzrecht

Zeitschrift für Zivilprozess 


\section{Literaturverzeichnis}

Ahrens, Martin

ders.

Andres, Dirk

Leithaus, Rolf

Arbeitskreis für Insolvenz- und Schiedsgerichtswesen e.V. (Hrsg.)

Arndt, Jürgen

Arnold, Egon

Meyer-Stolte, Klaus

Herrmann, Karl-Otto

Hansens, Heinz

Rellermeyer, Klaus

Bähr, Rainer M.

Balz, Manfred
Anmerkung zu BGH, Beschluss vom 17.03.2005 - IX ZB 214/04

in: Neue Zeitschrift für das Recht der Insolvenz und Sanierung (NZI) 2005, S.401-403

Kurzkommentar zu BGH, Urteil vom 18.05.2006 - IX ZR 187/04

in: Entscheidungen zum Wirtschaftrecht (EWiR) 2006, S.539-540

\section{Insolvenzordnung}

Kommentar, 1.Auflage, München 2006

zitiert: Andres/Leithaus/Bearbeiter

Kölner Schrift zur Insolvenzordnung

Das neue Insolvenzrecht in der Praxis

2. Auflage, Berlin 2000

zitiert: KS/Bearbeiter

Rechtspflegergesetz

Kommentar, Berlin /Frankfurt a.M. 1957

zitiert: Arndt, RpflG 1957

Rechtspflegergesetz

Kommentar, 6.Auflage, Bielefeld 2002

zitiert: Arnold/Bearbeiter, RpflG

Forderungsprüfung und Tabellenführung

in: Insolvenz und Vollstreckung (InVo) 1998, S.205-213

Kommentar zu BAG, Urteil vom 13.06.1989 - 1 AZR 819/87

in: Entscheidungen zum Wirtschaftsrecht (EWiR) 1989, S.745-746 
Bauer, Joachim

Baumbach, Adolf

Lauterbach, Wolfgang

Albers, Jan

Hartmann, Peter

Baur, Fritz

Stürner, Rolf

Baur, Fritz

Stürner, Rolf

Bruns, Alexander

Beck, Siegfried

Depré, Peter

Becker, Christoph

Bessenge, Peter

Herbst, Gerhard

Bessenge, Peter

Herbst, Gerhard

Roth, Herbert
Ungleichbehandlung der Gläubiger im geltenden Insolvenzrecht

in: Deutsche Zeitschrift für Wirtschafts- und Insolvenz recht (DZWir) 1998, S.188-192

Zivilprozessordnung mit Gerichtsverfassungsgesetz und anderen Nebengesetzen, Kommentar

56. Auflage, München 1998

67. Auflage, München 2009

zitiert: Baumbach/Lauterbach/Albers/Hartmann, ZPO (1998/2008)

Zwangsvollstreckungs-, Konkurs- und Vergleichsrecht

Band II, Insolvenzrecht, 12. Auflage,

Heidelberg 1990

zitiert: Baur/Stürner, Insolvenzrecht

\section{Zwangsvollstreckungsrecht}

13. Auflage, Heidelberg u.a. 2006

zitiert: Baur/Stürner/Bruns, Zwangsvollstreckung

Praxis der Insolvenz

Ein Handbuch für die Beteiligten und ihre Berater München 2003

zitiert: Beck/Depré/Bearbeiter

Insolvenzrecht

2. Auflage, Köln u.a. 2008

Gesetz über die Angelegenheiten der freiwilligen Gerichtsbarkeit / Rechtspflegergesetz Kommentar, 7. Auflage, Heidelberg 1995 zitiert: Bessenge/Herbst, RpflG

Gesetz über die Angelegenheiten der freiwilligen Gerichtsbarkeit / Rechtspflegergesetz

Kommentar, 9. Auflage, Heidelberg 2002

zitiert: Bessenge/Herbst/Roth, RpflG 
Birkenhauer, Ulf

Blersch, Jürgen

Goetsch, Hans- Wilhelm

Haas, Ulrich

Bley, Erich

Mohrbutter, Jürgen

Bötticher, Eduard

Bratvogel, Gerhard

Braun, Eberhard

Breuer, Wolfgang

Brückl, Daniel
Probleme der Nichtteilnahme am und im Insolvenzverfahren

Köln u.a. 2002

zugleich: Diss. Univ. Augsburg, 2000

Berliner Kommentar zum Insolvenzrecht

Kommentierung der InsO und der InsVV mit Antragsmustern für die Rechtspraxis

Loseblatt, Stand: 2009, Freiburg

zitiert: BK/Bearbeiter

Vergleichsordnung

Kommentar, Band II §§ 82-132, 3. Auflage

Berlin 1972

zitiert: Bley/Mohrbutter, VglO

Funktionelle und instrumentale Züge des Konkursverfahrens

in: Zeitschrift für Zivilprozess $\quad$ (ZZP) 1973 (Band 86), S.373-392

In welchen Fällen muß der Konkursverwalter die Termine vor dem Konkursgericht persönlich wahrnehmen, in welchen Fällen kann er sich durch einen Bevollmächtigten vertreten lassen, und wann kann und muß ein anderer Konkursverwalter bestellt werden?

in: Konkurs-, Treuhand- und Schiedsgerichtswesen (KTS) 1977, S.229-232

Insolvenzordnung

Kommentar, 3. Auflage, München 2007

zitiert: Braun/Bearbeiter

Insolvenzrecht

2. Auflage, München 2003

zitiert: Breuer, Insolvenzrecht

Die Forderung aus vorsätzlich begangener unerlaubten Handlung in der Insolvenz des Schuldners

in: Zeitschrift für das gesamte Insolvenzrecht (ZInsO) 2005, S.16-20 
Bund Deutscher Rechtspfleger e.V.

Burgermeister, Udo

Carl, Oliver

Dästner, Christian

Dauner-Lieb, Barbara

Heidel, Thomas

Ring, Gerhard

Hüßtege, Rainer

Mansel, Heinz-Peter

Noak, Ulrich

Eckardt, Diederich

Eckardt, Diederich

Eickmann, Dieter
Rechtspflegergesetz

Rechtspflegerblatt, Düsseldorf 1957

Der Sicherheitenpool im Insolvenzrecht

2. Auflage, Köln 1996

zitiert: Burgermeister, Sicherheitenpool

Teilnahmerechte im Konkurs

Gerichtliche Verfahrensleitung, Rechte der beteiligten und ihr Schutz im Insolvenzverfahren

Münster 1998

zugleich: Diss. Univ. Halle, 1998

zitiert: Carl, Teilnahmerechte

Neue Formvorschriften im Prozessrecht

in: Neue Juristische Wochenschrift (NJW) 2001, S.3469-3471

Anwaltkommentar BGB

Band I: Allgemeiner Teil mit EGBGB

Bonn 2005

zitiert: AnwkBGB-Bearbeiter

Die Feststellung und Beendigung des Insolvenzgläubigerrechts

in: Kölner Schrift zum Insolvenzrecht 2002, S.743-780

zitiert: KS/Eckardt

„Unanmeldbare“ Forderungen im Konkursfeststellungsverfahren nach §§ 138ff. KO

in: Zeitschrift für Wirtschaftsrecht (ZIP) 1993, S.1765-1775

Zweifelsfragen aus dem Konkursverfahren

in: Der Deutsche Rechtspfleger (Rpfleger) 1970, S.318-322 
Eickmann, Dieter

Eisner, Helmut

Gaul, Hans Friedhelm

Gerbers, Axel

Pape, Gerhard

Gerhardt, Walter

Gerke, Lars

Gogger, Martin

Gottwald, Peter
Höchstpersönliches Verwalterhandeln oder Delegation?

in: Konkurs-, Treuhand- und Schiedsgerichtswesen (KTS) 1986, S.197-207

Der isolierte Widerspruch des Schuldners gegen eine Forderung aus unerlaubter Handlung

in: Neue Zeitschrift für das Recht der Insolvenz und Sanierung (NZI) 2003, S.480-486

Negative Rechtskraftwirkung und konkursmäßige Zweittitulierung

in: Festschrift für Friedrich Weber zum 70. Geburtstag, S.155-179

Hrsg.: Bökelmann, Erhard/ Henckel, Wolfram/ Jahr, Günther

zitiert: Gaul, in: FS/Weber

Der Umgang mit Forderungsanmeldungen nach Einreichung des Schlussberichts

in: Zeitschrift für das gesamte Insolvenzrecht (ZInsO) 2006, S.685-690

Grundbegriffe des Vollstreckungs- und Insolvenzrechts

Stuttgart u.a. 1985

zitiert: Gerhardt, Grundbegriffe

Uneingeschränkte Dispositionsmaxime beim Gläubigerantrag trotz vorläufiger Verwaltung?

in: Zeitschrift für das gesamte Insolvenzrecht (ZInsO) 2003, S.873-881

Insolvenzrecht

2. Auflage, München 2006

Insolvenzrechtshandbuch

München 1990

zitiert: Gottwald/Bearbeiter, KO

3. Auflage, München 2006

zitiert: Gottwald/Bearbeiter 
Graf-Schlicker, Marie Luise

Grothe, Helmut

Gundlach, Ulf

Frenzel, Volker

Haarmeyer, Hans

Wutzke, Wolfgang

Förster, Karsten

dies.

Haarmeyer, Hans

Seibt, Werner

Hahn, Carl

Hahn, Carl

Hartmann, Peter
Kommentar zur Insolvenzordnung

Köln 2007

zitiert: Graf-Schlicker/Bearbeiter

Fremdwährungsverbindlichkeiten

Berlin 1999

Anmerkung zu BGH, Beschluss vom 22.03.2007 - IX ZB 8/05

in: Neue Zeitschrift für das recht der Insolvenz und Sanierung (NZI) 2007, S.402-403.

Handbuch zur Insolvenzordnung InsO/EGInsO

3. Auflage, München 2001

Kommentar zur Gesamtvollstreckungsordnung 4. Auflage, Köln 1998

zitiert: Haarmeyer/Wutzke/Förster, GesO

Akteneinsicht durch Gläubiger und „Dritte“ im Insolvenzverfahren

in: Der Deutsche Rechtspfleger (Rpfleger) 1996, S.221-227

Die gesammten Materialien zur Konkursordnung Berlin 1881

zitiert: Hahn, KO

Die gesammten Materialien zu dem Gerichtsverfassungsgesetz

Berlin 1879

zitiert: Hahn, GVG

Zivilprozess 2001/2002: Hundert wichtiger Änderungen

in: Neue Juristische Wochenschrift (NJW) 2001, S.2577-2598 
Häsemeyer, Ludwig

Hattwig, Stefan

Richter, Claus

Heeseler, Markus

Heil, Christof

Heinemann, Jörn

Henckel, Wolfram

Gerhardt, Walter

Henckel, Wolfram
Insolvenzrecht

Köln u.a. 1992

zitiert: Häsemeyer, KO

4. Auflage, Köln u.a. 2007

zitiert: Häsemeyer, InsO

Die Behandlung von Widersprüchen des Schuldners gegen eine durch Vollstreckungsbescheid titulierte Forderung aus vorsätzlich begangener unerlaubter Handlung

in: Zeitschrift für Verbraucher- und PrivatInsolvenzrecht (ZVI) 2006, 373-380

Auskunfts-/Akteneinsichtsrecht und weitere Informationsmöglichkeiten des Gläubigers im Regelinsolvenzverfahren

in: Zeitschrift für das gesamte Insolvenzrecht (ZInsO) 2001, S.873-887

Akteneinsicht und Auskunft im Konkurs Stuttgart 1995

zugleich: Diss. Univ. Jena, 1993

Neubestimmung der prozessualen Schriftform

Köln u.a. 2002

zugleich: Diss. Univ. Erlangen-Nürnberg, 2001

Jaeger - Insolvenzordnung

Großkommentar

Band 1, §§ 1-55 InsO, Berlin 2004

Band 2, §§ 56-102 InsO, Berlin 2007

zitiert: Jaeger/Bearbeiter, InsO

Der Gegenstand des Verfahrens zur Feststellung von Konkursforderungen

in: Festschrift für Karl Michaelis, S.151-172

Hrsg.: Pawlowski, Hans-Martin/ Wieacker, Franz Göttingen 1972

zitiert: Henckel, in: FS/Michaelis 
Henckel, Wolfram

ders.

Henning, Kai

Hess, Harald

Pape, Gerhard

Hess, Harald

Weis, Michaela

Wienberg, Rüdiger

Hess, Harald

ders.

Hattwig, Stefan

Hofer, Robert
Prozessrecht und materielles Recht

Göttingen 1970

zitiert: Henckel, Prozessrecht

Parteilehre und Streitgegenstand im Zivilprozess

Heidelberg 1961

zugleich: Habil. Univ. Heidelberg, 1959

zitiert: Henckel, Parteilehre

Aktuelles zu Überschuldung und Insolvenzen natürlicher Personen

in: Zeitschrift für das gesamte Insolvenzrecht (ZInsO) 2004, S.585-594

InsO und EGInsO: Grundzüge des neuen Insolvenzrechts

Köln 1995

zitiert: Hess/Pape/Bearbeiter

Kommentar zur Insolvenzordnung mit EGInsO

Band I, 2. Auflage, Heidelberg 2001

zitiert: Hess/Weis/Wienberg/Bearbeiter

Insolventrecht - Großkommentar in drei Bänden

Band I, §§ 1-112 InsO, Heidelberg 2007

Band II, §§ 113-359 InsO, Heidelberg 2007

zitiert: Hess, InsO

Kommentar zur Konkursordnung

6. Auflage, Neuwied/Kriftel 1997

zitiert: Hess/Bearbeiter, KO

Ungewissheit für Schuldner deliktischer Forderungen - Überlegung zu § 184 InsO

in: Zeitschrift für das gesamte Insolvenzrecht (ZInsO) 2004, S.636-641

Neue Aspekte zum Feststellungsvermerk im Insolvenzverfahren

in: Der Deutsche Rechtspfleger (Rpfleger) 2007, S.361-365 
Hofmann, Paul

Kersting, Friedrich

Holzer, Johannes

Huntemann, Eva Maria

Brockdorff, Christian Graf

Jaeger, Ernst

ders.

Jaeger, Ernst

Henckel, Wolfram

Jauernig, Othmar

Kahlert, Henner

Kahlert, Sven

Breutigam, Axel
Rechtspflegergesetz

Berlin 1957

zitiert: Hofmann/Kersting, RpflG 1957

Redaktionsversehen in der Insolvenzordnung?

in: Neue Zeitschrift für das Recht der Insolvenz und Sanierung (NZI) 1999, S.44-47

Die Gläubiger im Insolvenzverfahren

Berlin 1999

zitiert: Huntemann/Brockdorff/Bearbeiter

Kommentar zur Konkursordnung und den Einführungsgesetzen

2. Auflage, Berlin 1904

zitiert: Jaeger, KO

Konkursordnung mit Einführungsgesetzen

Band II, 1. Halbband, §§ 71-206 KO

Kommentar, 8. Auflage, Berlin 1973

zitiert: Jaeger/Bearbeiter, KO

Konkursordnung - Großkommentar

9. Auflage, §§ 1-42 KO, Berlin 1997

zitiert: Jaeger/Henckel, KO

Zwangsvollstreckungs- und Insolvenzrecht

22. Auflage, München 2007

Beseitigung des Widerspruchs des Schuldners gegen den Haftungsgrund der vorsätzlichen unerlaubten Handlung im Insolvenzverfahren

in: Zeitschrift für das gesamte Insolvenzrecht (ZInsO) 2006, S.409-413

Forderungsfeststellung im Planverfahren - eine unendliche Geschichte?

in: Zeitschrift für das gesamte Insolvenzrecht (ZInsO) 2002, S.469-472 
Kaiser, Sven

Crämer, Claudia

Kehe, Thomas

Meyer, Lars-Michael

Schmerbach, Ulrich

Keller, Ulrich

Kemper, Jutta

Kilger, Joachim

Kirchhof, Hans-Peter

Lwowski, Hans-Jürgen

Stürner, Peter

Klasmeyer, Bernd

Elsner, Ben
Originale - das einzig Wahre?

in: Insolvenz und Vollstreckung (InVo) 2001, S.153-156

Anmeldung und Feststellung einer Forderung aus unerlaubter Handlung - Teil 1 und 2

in: Zeitschrift für das gesamte Insolvenzrecht (ZinsO) 2002, S.615-618 und S.660-667

Insolvenzrecht

München 2006

Die Verordnung (EG) Nr.1346/2000 über Insolvenzverfahren

in: Zeitschrift für Wirtschaftsrecht (ZIP) 2001, S.1609-1621

Vergleichsordnung

11. Auflage, München 1986

zitiert: Böhle-Stamschräder/Kilger

Münchner Kommentar zur Insolvenzordnung

Band 1, §§ 1-102 InsO, 2. Auflage, München 2007

Band 2, §§ 103-269 InsO, 2. Auflage, München 2008

Band 3, §§ 270-359 InsO, 2. Auflage, München 2008 zitiert: MüKo-InsO/Bearbeiter

Zur Behandlung von Ausfallforderungen im Konkurs

in: Festschrift für Franz Merz zum 65. Geburtstag, S.303-311

Hrsg.: Gerhardt, Walter/ Henckel, Wolfram/ Kilger, Joachim/ Kreft, Gerhardt

Köln 1992

zitiert: Klasmeyer/Elsner, in: FS/Merz

Verfahrenskostenstundung, Restschuldbefreiung und Verbraucherinsolvenzverfahren

Kommentar, 3. Auflage, Neuwied 2006

zitiert: Grote/Ahrens/Grote/Bearbeiter 
Köster, Malte

Ahrendt, Achim

Kreft, Gerhart

Kübler, Bruno M.

Kübler, Bruno M.

Prütting, Hanns

Bork, Reinhard

Kuhn, Georg

Uhlenbruck, Wilhelm

Landfermann, Hans-Georg

Mandlik, Gerhard
Kommentar zu BGH, Urteil vom 01.12.2005 - IX ZR 95/04 (LG Berlin)

in: Entscheidungen zum Wirtschaftsrecht (EWiR) 2006, S.177-178

Heidelberger Kommentar zur Insolvenzordnung 5. Auflage, Heidelberg 2008 zitiert: HK/Bearbeiter

Zur Abgrenzung der Zuständigkeit von Gesamtvollstreckungsgericht und Verwalter bei der Feststellung der Schuldenmasse

in: Festschrift für Wolfram Henckel zum 70 . Geburtstag, S.495-513

Hrgs: Gerhardt, Walter/ Diederichsen, Uwe/ Rimmelspacher, Bruno/ Costede, Jürgen Berlin 1995

zitiert: Kübler, in: FS/Henckel

\section{Kommentar zur Insolvenzordnung}

Band I, §§ 1-79 InsO

Band II, §§ 80-173 InsO

Band III, §§ 174-359 InsO

Loseblatt, Stand: 2009, Köln

zitiert: Kübler/Prütting/Bork/Bearbeiter

\section{Konkursordnung}

Kommentar, 11. Auflage, München 1994

zitiert: Kuhn/Uhlenbruck, KO

Allgemeine Wirkungen der Insolvenzeröffnung

in: Kölner Schrift zur Insolvenzordnung 2002, S.169-192

zitiert: KS/Landfermann

Feststellungsvermerk bei Ausfallforderungen im Konkurs

in: Der Deutsche Rechstpfleger (RPfleger) 1980, S.143-144 
Mäusezahl, Uwe

Merkle, Rupert

Mohrbutter, Harro

Ringstmeier, Andreas

Münzel, Karl

Musielak, Hans-Joachim

Säcker, Franz Jürgen

Rixecker, Roland

Nerlich, Jörg

Römermann, Volker

Oelrichs, Carsten
Die unerlaubte Handlung in der Insolvenz der natürlichen Person

in: Zeitschrift für das gesamte Insolvenzrecht (ZinsO) 2002, S.462-468

Die Zuständigkeit von Insolvenzverwalter und Insolvenzgericht im insolvenzrechtlichen Feststellungsverfahren

in: Der Deutsche Rechtspfleger (RPfleger) 2001, S.157-166

Handbuch der Insolvenzverwaltung

8. Auflage, Köln/Berlin/München 2007

zitiert: Mohrbutter/Ringstmeier/Bearbeiter

Freiwillige Gerichtsbarkeit und Zivilprozeß in der neueren Entwicklung

in: Zeitschrift für Zivilprozess (ZZP) 1953 (Bd.66), S.334-386

Kommentar zur Zivilprozessordnung

6. Auflage, München 2008

zitiert: Musielak/Bearbeiter, ZPO

Münchener Kommentar zum Bürgerlichen

Gesetzbuch

Band 1, Allgemeiner Teil (§§ 1-240 BGB), 5. Auflage, München 2006

Band 2, Schuldrecht, Allgemeiner Teil (§§ 244-432 BGB), 3. Auflage, München 1994

Band 2, Schuldrecht, Allgemeiner Teil (§§ 241-432 BGB), 5. Auflage, München 2007

zitiert: MüKo-BGB/Bearbeiter

Insolvenzordnung

Kommentar, Loseblatt, Stand: 2009, München

zitiert: Nerlich/Römermann/Bearbeiter

Gläubigermitwirkung und Stimmverbote im neuen Insolvenzrecht

Köln u.a. 1999

zugleich: Diss. Univ. Hamburg, 1998

zitiert: Oelrichs, Gläubigermitwirkung 
Pape, Gerhard

Uhlenbruck, Wilhelm

Pape, Gerhard

ders.

ders.

ders.

ders.

ders.

ders.
Insolvenzrecht

München 2002

zitiert: Pape/Uhlenbruck, InsO

Die Eigenverwaltung des Schuldners nach der Insolvenzordnung

in: Kölner Schrift zur Insolvenzordnung 2002, S.895-929

zitiert: KS/Pape

Zur Problematik der Unanfechtbarkeit von Stimmrechtsfestsetzungen in der Gläubigerversammlung

in: Zeitschrift für Wirtschaftsrecht (ZIP) 1991, S.837-850

Die Gläubigerautonomie in der Insolvenzordnung in: Zeitschrift für das gesamten Insolvenzrecht (ZInsO) 1999, S.305-316

Recht auf Einsicht in Konkursakten - ein Versteckspiel für die Gläubiger?

in: Zeitschrift für Wirtschaftsrecht (ZIP) 1991, S.1367-1371

Zur Konkurrenz der Vollstreckung aus einer Eintragung in die Konkurstabelle und einem vor Konkurseröffnung erwirkten Titel

in: Zeitschrift für Insolvenzrecht (KTS) 1992, S.185-190

Akteneinsicht für Insolvenzgläubiger - Ein ständiges Ärgernis

in: Zeitschrift für Wirtschaftsrecht (ZIP) 2004, S.598-605

Anmerkung zu BGH, Beschluss vom 17.03.2005 - IX ZB 214/04

in: Zeitschrift für das gesamten Insolvenzrecht (ZInsO) 2005, S.599-600 
Pape, Gerhard

Palandt, Otto

Peters, Frank

Prütting, Hanns

Prütting, Hanns

Wegen, Gerhard

Weinreich, Gerd

Rauscher, Thomas

Wax, Peter

Wenzel, Joachim

Ripka, Bernd

Schmerbach, Ulrich
Vorzeitige Erteilung der Restschuldbefreiung bei fehlenden Forderungsanmeldungen

in: Neue Zeitschrift für das Recht der Insolvenz und Sanierung (NZI) 2004, S.1-7

Bürgerliches Gesetzbuch

68. Auflage, München 2009

zitiert: Palandt/Bearbeiter

Die Forderung aus einer vorsätzlich begangenen unerlaubten Handlung in der Einzelvollstreckung und in der Insolvenz

in: Zeitschrift für Insolvenzrecht (KTS) 2006, S.127-133

Allgemeine Verfahrensgrundsätze der Insolvenzordnung

in: Kölner Schrift zur Insolvenzordnung 2002, S.221-247

zitiert: KS/Prütting

\section{BGB Kommentar}

3. Auflage, Neuwied 2008

zitiert: Prütting/Wegen/Weinreich/Bearbeiter

Münchener Kommentar zur Zivilprozessordnung mit Gerichtsverfassungsgesetz und Nebengesetzen Band 2, §§ 511-945 ZPO, 3. Auflage, München 2007 zitiert: MüKo-ZPO/Bearbeiter

Poolverträge und die neuere Entwicklung des Gesellschaftsrecht

Herdecke 2000

zugleich: Diss. Univ. Tübingen, 2000

zitiert: Ripka, Poolverträge

Anmerkung zu AG Göttingen, Beschluss vom 04.03.2002 - 74 IN 60/02

in: Zeitschrift für das gesamte Insolvenzrecht (ZinsO) 2002, S.292-293. 
Schmidt, Andreas

Schmidt, Karsten

ders.

Schmidt, Thomas B.

Seuffert, Lothar

Smid, Stefan

Smid, Steffan

ders.
Hamburger Kommentar zum Insolvenzrecht

2. Auflage, Münster 2007

zitiert: HHK/Bearbeiter

Insolvenzgesetze $\mathrm{KO} / \mathrm{VglO} / \mathrm{GesO}$

17. Auflage, München 1997

zitiert: Kilger/K.Schmidt, KO

Das Insolvenzrecht und seine Reform zwischen Prozeßrecht und Unternehmensrecht

in: Konkurs-, Treuhand- und Schiedsgerichtswesen (KTS) 1988, S.1-18

Das Prüfungsrecht des Insolvenzverwalters zum Forderungsgrund der unerlaubten Handlung

in: Zeitschrift für das gesamte Insolvenzrecht (ZinsO) 2006, S.523-526

Kommentar zur Civilprozeßordnung für das Deutsche Reich

Nördlingen 1879

zitiert: Seuffert, ZPO 1877

8. Auflage, 1. Band, München 1902

zitiert: Seuffert, ZPO 1898

Grundzüge des Insolvenzrechts

4. Auflage, München 2002

zitiert: Smid, Grundzüge

Insolvenzordnung (InsO) mit insolvenzrechtlicher Vergütungsordnung (InsVV)

2. Auflage, Stuttgart 2001

zitiert: Smid/Bearbeiter

Der Forderungsanmeldung beizufügende Belege - Anmerkung zu BGH Urteil IX ZR 95/04 vom 01.12.2005

in: JurisPR-InsR 7/2006, Anm. 3 
Spellenberg, Ulrich

Staudinger, Julius von

Stein, Friedrich

Jonas, Martin

Thomas, Heinz

Putzo, Hans

Tscheschke, Brigitte

Uhlenbruck, Wilhelm

Uhlenbruck, Wilhelm

Vallender, Heinz
Zum Gegenstand des Konkursfeststellungsverfahrens ( $\S \S 138 f f \mathrm{KO}$ )

Göttingen 1973

zugleich: Diss. Univ. Göttingen, 1973

zitiert: Spellenberg, Gegenstand

Kommentar zum Bürgerlichen Gesetzbuch mit Einführungsgesetz und Nebengesetzen

Buch 1, Allgemeiner Teil (5), §§ 164-240 BGB, Berlin 2004

zitiert: Staudinger/Bearbeiter

Kommentar zur Zivilprozessordnung

Band 3, §§ 128-252 ZPO, 22. Auflage, Tübingen 2005

Band 4, §§ 253-327 ZPO, 22. Auflage, Tübingen 2008

zitiert: Stein/Jonas/Bearbeiter, ZPO

\section{Zivilprozessordnung}

29. Auflage, München 2008

zitiert: Thomas/Putzo/Bearbeiter, ZPO

Nachträglich angemeldete Forderungen in der Konkursabwicklung

in: Der deutsche Rechtspfleger (Rpfleger) 1992, S.96-98

Insolvenzordnung

Kommentar, 12. Auflage, München 2003

zitiert: Uhlenbruck/Bearbeiter

Zurückweisung von Anmeldungen zur Konkurstabelle

in: Der deutsche Rechtspfleger (Rpfleger) 1991, S.445-447

Auswirkungen des Schuldrechtsmodernisierungsgesetzes auf die Anmeldung von Forderungen im Insolvenzverfahren

in: Zeitschrift für das gesamten Insolvenzrecht (ZInsO) 2002, S.110-112 
Vallender, Heinz

Vollkommer, Max

Wellensiek, Jobst

Westermann, Harm Peter

Wilmowski, Gustav von

Kurlbaum, Karl

Wimmer, Klaus

Wagner, Martin

Dauernheim, Jörg

Weidekind, Sabine-Sophie

Wimmer, Klaus

ders.

Wolff, Theodor
Das rechtliche Gehör im Insolvenzverfahren

in: Kölner Schrift zum Insolvenzrecht 2002, S.249-284

zitiert: KS/Vallender

Formstrenge und prozessuale Billigkeit

München 1973

zugleich: Habil. Univ. München 1973

Die Aufgaben des Insolvenzverwalters nach der Insolvenzordnung

in: Kölner Schrift zum Insolvenzrecht 2002, S.403-429

zitiert: KS/Wellensiek

Erman - Bürgerliches Gesetzbuch

11. Auflage, Münster/Köln 2004

zitiert: Erman/Bearbeiter

Kommentar zur Konkursordnung

6. Auflage, Berlin/Vahlen 1906

zitiert: Wilmowski/Kurlbaum, KO

Handbuch des Fachanwalts Insolvenzrecht

3. Auflage, München 2008

zitiert: Handbuch-InsO/Bearbeiter

Frankfurter Kommentar zur Insolvenzordnung

5. Auflage, Neuwied, Kriftel 2009

zitiert: FK/Bearbeiter

Die Verordnung (EG) Nr.1346/2000 über Insolvenzverfahren

in: Zeitschrift für das gesamte Insolvenzrecht (ZInsO) 2001, S.97-103

Konkursordnung mit Einführungsgesetz, Nebengesetzen und Ergänzungen in der Fassung der Gesetze vom 17. Mai 1898

Kommentar, 2. Auflage, Berlin/Leipzig 1921

zitiert: Wolff, KO 
Wunner, Christian

Schuster, Michael

Zöller, Richard
Zum Jahresende: Die Hemmung der Verjährung durch die Anmeldung von Forderungen im Insolvenzverfahren

in: Der Betriebs-Berater (BB) 2006, S.2649-2653

Zöller - Zivilprozessordnung

Kommentar, 27. Auflage, Köln 2009

zitiert: Zöller/Bearbeiter, ZPO 


\section{$\underline{\text { A. Einleitung }}$}

Die vorliegende Arbeit befasst sich mit dem Anmelde- und Feststellungsverfahren von Insolvenzforderungen im Insolvenzverfahren gemäß den $\S \S 174 f f$. InsO, welches in den letzten Jahren seit Einführung der Insolvenzordnung durch eine Vielzahl von Gerichtsentscheidungen und Literaturmeinungen geprägt worden ist.

Ausgehend vom Sinn und Zweck des Insolvenzverfahrens soll das Ziel der vorliegenden Arbeit eine einheitliche Analyse der Zusammenhänge zwischen dem Ablauf des Anmelde- und Feststellungsverfahrens und der damit verbundenen Begründung und Ausübung sowie des Verlustes von Verfahrensrechten für die Verfahrensbetroffenen und Verfahrensbeteiligten sein. Dabei sollen nicht nur die einzelnen Voraussetzungen zur Begründung und Ausübung der unterschiedlichen Verfahrensrechte im Allgemeinen und innerhalb der jeweiligen Verfahrensabschnitte herausgearbeitet und festgestellt, sondern insbesondere deren mögliche Auswirkungen auf das Insolvenzverfahren sowie auf die Rechte des einzelnen Verfahrensbeteiligten und der übrigen Verfahrensbetroffenen erörtert werden. Die Arbeit soll damit einen Beitrag zur einheitlichen Durchführung des Anmelde- und Feststellungsverfahrens in Bezug zu den in diesem Verfahren verfolgten unterschiedlichen Interessen der Beteiligten leisten und die dabei aufgezeigten derzeitigen Defizite im Einzelfall einer Lösung zuführen.

Im Rahmen dieser weitgehend an den chronologischen Ablauf des Insolvenzverfahrens angelehnten Analyse soll insbesondere aufgezeigt werden, dass der entscheidende Anknüpfungspunkt zur Begründung und für den Verlust von weitreichenden Verfahrensrechten im Insolvenzverfahren stets die formelle Insolvenzgläubigerstellung ist, die nur durch eine ordnungsgemäße Insolvenzforderungsanmeldung erreicht werden kann. Deshalb wird zunächst zu klären sein, ab und bis zu welchem Zeitpunkt eine Anmeldung überhaupt erfolgen kann und wie diese nach Form und Inhalt beschaffen sein muss, um zum Insolvenzverfahren zugelassen zu werden. Dies beinhaltet die Frage, wer in welchem Umfang wann die Anmeldungen auf ihre Zulässigkeit zu prüfen hat und inwiefern 
diese formelle Prüfung der Anmeldung von der materiellrechtlichen Prüfung der in der Anmeldung enthaltenen Insolvenzforderung zu trennen ist. Nach der grundsätzlichen Erörterung der verfahrensrechtlichen Wirkung der ordnungsgemäßen Anmeldung soll es Schwerpunkt dieser Arbeit sein, vor dem Hintergrund der Gläubigerautonomie im insolvenzgerichtlich geleiteten Verfahren insbesondere die gegenseitige Einflussmöglichkeit der Insolvenzgläubiger untereinander im Rahmen der materiellrechtlichen Prüfung aller angemeldeten Insolvenzforderungen im Prüfungstermin und den sonstigen Gläubigerversammlungen durch Ausübung des mit der ordnungsgemäßen Anmeldung begründeten Widerspruchrechts auf das darüber hinaus eröffnete Stimm- und Teilhaberecht an der Insolvenzmasse zu untersuchen. Dazu werden den in den einzelnen Verfahrensstadien und -situationen verfolgten Insolvenzgläubigerinteressen die Interessen des Schuldners und die durch den Insolvenzverwalter wahrgenommene Gesamtinteressenvertretung gegenübergestellt, um so die Möglichkeiten eines umfassenden Interessenausgleichs bezüglich der Wahrnehmung der jeweiligen Verfahrensrechte im Insolvenzverfahren analysierend aufzuzeigen.

Neben der Erörterung der unterschiedlichen Ausübungsmöglichkeiten eines Widerspruchs durch die Verfahrensteilnehmer und seiner Wirkung auf das Verfahren und die Verfahrensrechte der betroffenen Insolvenzforderungsinhaber werden insbesondere Interessenausgleichsdefizite hinsichtlich der Verfolgung von Widersprüchen im Feststellungsprozess vor allem im Zusammenhang mit der Nachhaftung des Schuldners durch Insolvenzforderungen aus vorsätzlich begangener unerlaubter Handlung und deren Auswirkungen für eine (mögliche) Restschuldbefreiung aufzuzeigen und einer Lösung zuzuführen sein.

Darüber hinaus werden der Zeitpunkt und der eigentliche Gegenstand der Feststellung sowie der damit verbundene Umfang der aus der Feststellung einer Insolvenzforderung erwachsenen Rechtskraftwirkung und deren Reichweite gegenüber den einzelnen Verfahrensbeteiligten zu erörtern sein. Hierbei wird insbesondere das Verhältnis des nachinsolvenzlich erteil- und vollstreckbaren Tabellenauszuges $\mathrm{zu}$ einem bereits bei Eröffnung des Insolvenzverfahrens vorliegenden Vollstreckungstitel über 
die (angemeldete/unangemeldete) Insolvenzforderung herauszuarbeiten und die verfahrensrechtlichen Folgen unter Berücksichtigung der jeweiligen Interessen der Insolvenzgläubiger und des Schuldners aufzuzeigen sein. 


\section{B. Stellung und Funktion des Anmelde- und Feststellungsverfahrens}

Nachdem das Insolvenzverfahren durch gerichtlichen Beschluss gem. $\S \S 27,28$ InsO eröffnet worden ist, stellt die Anmeldung zur Insolvenztabelle gem. $\S 174 \mathrm{InsO}^{1}$ für jeden Insolvenzgläubiger (§ 38 InsO) der einzige gesetzlich vorgesehene Weg dar, am Insolvenzverfahren teilnehmen und mitwirken zu können, um seine persönlichen Forderungen gegen den insolventen Schuldner geltend zu machen.

Das Verfahren der $\S \S 174 \mathrm{ff}$. InsO ist eine Konkretisierung des $\S 87$ InsO, der zur Verfolgung von Insolvenzforderungen nach Verfahrenseröffnung die ausschließliche Anwendung der Insolvenzordnung vorschreibt und dadurch dem Insolvenzgläubiger die Durchsetzung seiner Forderung im Wege der ordentlichen Gerichtsbarkeit außerhalb der Insolvenzordnung verwehrt. ${ }^{2}$ Indem sämtliche Insolvenzforderungen gegen den Schuldner den gleichen verfahrensrechtlichen Vorschriften der $\S \S 174 \mathrm{ff}$. InsO unterworfen werden, ${ }^{3}$ soll gegenüber dem früheren $\S 12 \mathrm{KO}$ Rechtsklarheit hinsichtlich der Durchsetzung von Forderungen während bzw. in der Insolvenz ${ }^{4}$ und eine weitgehende Gleichstellung der Insolvenzgläubiger geschaffen werden. ${ }^{5}$ Damit weicht das allgemeine Prioritätsprinzip der Einzelvollstreckung einem unter gerichtlicher Aufsicht stehenden Gesamtvollstreckungsverfahren. ${ }^{6}$

Dies hat zur Folge, dass der Insolvenzgläubiger nicht nur zur Geltendmachung seines sich aus $\S 38$ InsO ergebenden Anspruchs auf Befriedigung aus der Insolvenzmasse am Anmelde- und

1 §§ 174ff. InsO entspricht im Grundsatz den vormaligen §§ 139ff. KO, auf deren Kommentierungen daher zurückgegriffen werden kann.

$2 \quad$ BGH ZIP 2003, 2379 (2382); FK/Kießner, § 174 Rn.7; Gottwald/Klopp/Kluth, § 19 Rn.1; Braun/Kießner, § 174 Rn.3.

3 Zugleich wird die Gleichstellung der Gläubiger untereinander gesichert.

4 Unter der Konkursordnung konnten die Gläubiger noch auf die Teilnahme am Konkursverfahren verzichten und ihre Forderungen außerhalb des Verfahrens durch Klage betiteln lassen. Lediglich die Vollstreckung während des Verfahrens war ausgeschlossen, §§ 12, $14 \mathrm{KO}$; siehe BGHZ 25, 395 (397ff.); 72, 234 (235) zur entsprechenden Auslegung des § 240 ZPO; Andres/Leithaus/Leithaus, § 87 Rn.1; Kuhn/Uhlenbruck, KO, § 12 Rn.4; Handbuch-InsO/Bruder, Rn.396; Häsemeyer, InsO, Rn.10.45; KS/Eckardt, S.749 Fn.21; Birkenhauer, S.54 m.w.N.

5 So ausdrücklich die Begr. zu § 98 (§ 87 InsO) RegE, BT-Drucks. 12/2443, S.137; MüKo-InsO/Breuer, $\S 87$ Rn.1; Kübler/Prütting/Bork/Lüke, $\S 87 \quad$ Rn.2; KS/Landfermann, S.168 Rn.31; Uhlenbruck/Uhlenbruck, § 87 Rn.12 m.w.N.

6 Uhlenbruck/Uhlenbruck, § 1 Rn.3; MüKo-InsO/Ganter, § 1 Rn.53; Häsemeyer, InsO, Rn.22.01; KS/Landfermann, S.169 Rn.33; Hess/Weis/Wienberg/Hess, Vor § 2 Rn.3, 8; Jaeger/Gerhardt, § 2 Rn.11; Breuer, Rn.396; Gogger, S.58. 
Feststellungsverfahren der $\S \S 174 f f$. InsO teilnehmen muss, sondern auch, um die durch die Eröffnung des Insolvenzverfahrens gem. § 240 ZPO unterbrochenen Rechtsstreitigkeiten ${ }^{7}$ (Schuldenmassestreit) ${ }^{8}$ oder seine

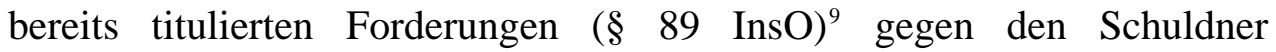
verfahrensrechtlich weiterverfolgen $\mathrm{zu}$ können. Insofern sind die Anmeldung der Insolvenzforderung zur Tabelle und der Widerspruch eines Widerspruchsberechtigten im Prüfungsverfahren notwendige Prozessvoraussetzung (vgl. §§ 179; 184 InsO). ${ }^{10}$

Hinsichtlich der Geltendmachung von Insolvenzforderungen dient das Anmelde- und Feststellungsverfahren der $\S \S$ 174ff. InsO durch die Zusammenführung sämtlicher Insolvenzforderungen in ein einheitliches Verfahren dazu, die jeweiligen Forderungen der Insolvenzgläubiger dem Grunde und der Höhe nach zu erfassen, nach ihrer dadurch ermöglichten Prüfung durch alle Verfahrensteilnehmer in vollstreckungsgeeigneter Form festzustellen und titulieren zu lassen (§§ 178 III, 201 II InsO). Neben diesem individuellen Interesse des einzelnen Insolvenzgläubigers an der Geltendmachung und Befriedigung seiner persönlichen Forderung gegen den Schuldner eröffnet ihm seine Stellung als Insolvenzgläubiger zugleich eine Reihe von anderen wichtigen, teilweise gemeinschaftlich mit den übrigen Verfahrensteilnehmern auszuübenden Teilnahmerechten ${ }^{11}$ im Insolvenzverfahren. Hierbei ist zwischen den Verfahrensrechten, die jedem materiellrechtlichen Insolvenzgläubiger zustehen und die an die bloße Eigenschaft als Insolvenzgläubiger gem. §§ 38, $39 \mathrm{InsO}^{12}$ anknüpfen, und den (zusätzlichen) wesentlich weiter reichenden Verfahrensrechten und

7 Die $\S \S 85,86$ InsO regeln die Wiederaufnahme von Aktiv- und Passivprozessen für Forderungen die keine Insolvenzforderungen sind und somit gerade keiner Anmeldung zum Verfahren der §§ 174ff. InsO bedürfen.

8 Zum Begriff siehe bei Häsemeyer, InsO, Rn.10.45 m.w.N.

$9 \quad \S \quad 89$ InsO legt ein generelles Vollstreckungsverbot während der Dauer des Insolvenzverfahrens fest. Damit soll ergänzend zu § 87 InsO ausgeschlossen werden, dass ein Gläubiger auf die Teilnahme am Insolvenzverfahren verzichtet und aufgrund eines gegen den Schuldner erwirkten Vollstreckungstitels im Übrigen die Zwangsvollstreckung gegen diesen betreibt und dadurch die Insolvenzmasse zum Nachteil der übrigen Insolvenzgläubiger schmälert. - Vgl. Begr. zu § 100 (§ 89 InsO) RegE, BT-Drucks. 12/2443, S.137.

10 BGH ZZP 75 (1962), 347 (349); OLG Brandenburg, Urteil v. 04.12.2007 6 U 109/06, S.9 (wohl unveröffentlicht); Nerlich/Römermann/Wittkowski, $§ \quad 87$ Rn.8; Kübler/Prütting/Bork/Lüke, § 87 Rn.1; Uhlenbruck/Uhlenbruck, § 87 Rn.7.

11 Dem Insolvenzgläubiger können Anhörungs-, Informations-, Prüfungs-, Antrags- und Beschwerderechte zustehen. - Vgl. Uhlenbruck/Uhlenbruck, § 74 Rn.7; ausführlich Oelrichs, Gläubigermitwirkung, S.27f., 61ff.

12 Zum absonderungsberechtigten Gläubiger als Insolvenzgläubiger gem. § 52 S.1 InsO siehe unter C.II. (S.16ff.). 
deren uneingeschränkten Ausübungsmöglichkeit zu unterscheiden, die - wie noch zu zeigen sein wird ${ }^{13}$ - nur vom formellen Insolvenzgläubiger nach ordnungsgemäßer Anmeldung und gegebenenfalls Feststellung seiner Insolvenzforderung im Verfahren der $\S \S 174 \mathrm{ff}$. InsO ausgeübt werden können.

So hat beispielsweise jeder - auch der nachrangige ${ }^{14}$ - Insolvenzgläubiger grundsätzlich das Recht auf Teilnahme und Gehör ${ }^{15}$ in der Gläubigerversammlung (vgl. § 74 I 2 InsO). ${ }^{16}$ Zur effektiven Durchsetzung und Wahrung seiner persönlichen Interessen, z.B. im Rahmen von Beschlussfassungen der Gläubigerversammlung, bedarf der Insolvenzgläubiger hingegen der generellen Feststellung seines Stimmrechts, ${ }^{17}$ die gem. $§ 77$ I 1 InsO die vorherige Anmeldung seiner Insolvenzforderungen voraussetzt. Dies hängt mit der Gläubigerautonomie $^{18}$ und der damit verbundenen Gewichtigkeit des Stimmrechts als konkretes Einwirkungsrecht nur des berechtigt teilnehmenden Insolvenzgläubigers während des gesamten Insolvenzverfahrens zusammen. Die Gläubigerautonomie wird durch die in der Gläubigerversammlung als dem zentralen insolvenzrechtlichen Selbstverwaltungsorgan ${ }^{19}$ gefassten Beschlüsse deutlich: Zum einen kann die Gläubigerversammlung organisatorisch auf das Verfahren Einfluss nehmen, indem sie Verfahrensregeln aufstellt (vgl. z.B. §§ 149 II, 271 S.1, 272 I Nr.1 InsO) und Insolvenzverwalter (§ 57 S.1 InsO) und Gläubigerausschuss (§ 68 I InsO) wählt. Zum anderen kann sie über die das Verfahren prägenden wirtschaftlichen Leitentscheidungen wie

\footnotetext{
Siehe unter C.I. (S.10ff.) und D.VI. (S.94ff.).

14 Vgl. Allgemeiner Teil des RegE zur InsO, BT-Drucks. 12/2443, S.99; Uhlenbruck/Uhlenbruck, § 74 Rn.7; MüKo-InsO/Ehricke, § 74 Rn.27; näher Pape, ZinsO 1999, 305 (308) m.w.N.

15 Näher dazu bei KS/Vallender, S.249ff.

16 Allgemeiner Teil des RegE zur InsO, BT-Drucks. 12/2443, S.99; siehe ferner MüKoInsO/Ganter, § 1 Rn.58 und Ehricke, § 74 Rn.27; Uhlenbruck/Uhlenbruck, § 74 Rn.10 jeweils m.w.N.

17 Sollte die Feststellung des Stimmrechts gem. § 77 InsO scheitern, so stehen dem Insolvenzgläubiger zumindest noch sein grundsätzliches Teilnahmerecht und sämtliche Antrags- und Beschwerderechte nach den §§ 78, 216, 251, 251 InsO zu. Siehe dazu unter E.I.4.e) (S.163ff.) - Begr. zu §§ 88, 89 (§§ 76, 77 InsO) RegE, BTDrucks. 12/2443, S.133f.; Oelrichs, Gläubigermitwirkung, S.28f.

18 KS/Prütting, S.244 Rn.72f.; Uhlenbruck/Uhlenbruck, § 1 Rn.13; Hess, InsO, Vor $\S 1$ Rn.263ff.; Pape/Uhlenbruck, InsO, Rn.128 m.w.N.

19 Hess, InsO, § 74 Rn.2; Kübler/Prütting/Bork/Kübler, § 74 Rn.3; Pape/Uhlenbruck, InsO, Rn.127; Uhlenbruck/Uhlenbruck, § 74 Rn.5; MüKo-InsO/Ehricke, § 74 Rn.2 jeweils m.w.N.
} 
Unternehmensfortführung, -zerschlagung, -sanierung u.ä. beschließen (vgl. z.B. §§ 157 S.1, 159, 235 I 1 InsO). ${ }^{20}$

Ebenso bedeutend für die formellen Verfahrensteilnehmer ist das Widerspruchsrecht im Prüfungstermin gegen die angemeldete und festzustellende Insolvenzforderung. ${ }^{21}$ Das Widerspruchsrecht ermöglicht nämlich dem formellen Insolvenzgläubiger neben dem Insolvenzverwalter der Berechtigung anderer Insolvenzgläubiger - mit ihren angemeldeten Insolvenzforderungen am Insolvenzverfahren teilzunehmen entgegenzutreten, was weitreichende Auswirkungen auf die Verfahrensrechte des Betroffenen hat. Ein Widerspruch hindert die Feststellung der angemeldeten Insolvenzforderung (§ 178 I, III InsO). Dies kann nicht nur zu einem Stimmrechtsverlust (vgl. § 77 I, II InsO) führen, sondern insbesondere zu einem Ausschluss von dem eigentlichen Ziel seiner Teilnahme: der Teilhabe am Verteilungsverfahren durch Ausschüttung der Quote. Denn letztendlich erlangt der Insolvenzgläubiger nur durch die Feststellung seiner Insolvenzforderung und ihrer Aufnahme ins Schlussverzeichnis das Recht, an der Ausschüttung der entfallenen Quote bei den Verteilungen teilzuhaben (vgl. § 189 II, III InsO). ${ }^{22}$ Darüber hinaus wird, wenn der Schuldner bei Eröffnung des Insolvenzverfahrens einen Antrag auf Restschuldbefreiung gestellt haben sollte (§ 287 I InsO), durch die Aufnahme der festgestellten Insolvenzforderung ins Schlussverzeichnis die rechtliche Grundlage für ihre weitere Berücksichtigung bei weiteren Verteilungen in der Wohlverhaltensphase geschaffen (§ 292 I 2 InsO). An die Berücksichtigung seiner Insolvenzforderung knüpft dann wiederum das Recht des Insolvenzgläubigers an, Versagungsanträge gegen eine Restschuldbefreiung des Schuldners $\mathrm{zu}$ stellen. Sollte der Insolvenzgläubiger daher seine Forderungen gar nicht erst zum Insolvenzverfahren anmelden oder aber seine angemeldete Insolvenzforderung nicht oder nicht rechtzeitig zur Aufnahme ins Schlussverzeichnis festgestellt werden, kann ihm auch das Recht, Versagungsanträge zu stellen, nicht zustehen. Denn er kann schon nicht mit dem Einwand gehört werden, seine Befriedigung - von der der 
Insolvenzgläubiger ja gerade ausgeschlossen ist - werde durch einen Verstoß des Schuldners gegen Obliegenheitspflichten beeinträchtigt. ${ }^{23}$

Wie sich zeigt, stellt die Zuerkennung des Teilnahmerechts und der damit verbundenen umfassenden Einflussnahme auf das Insolvenzverfahren für den Insolvenzgläubiger ein gleichgewichtiges Interesse dar, wie die eigentliche Befriedigung seiner Insolvenzforderung. Das Eine geht dem Anderen voraus. Beides ist daher unmittelbar miteinander verbunden.

Das Anmelde- und Feststellungsverfahren der $\S \S 174 \mathrm{ff}$. InsO regelt also nicht nur die Voraussetzungen zur Geltendmachung von Insolvenzforderungen im Insolvenzverfahren, sondern ist auch Ausgangspunkt für die Begründung weiterer Teilnahmerechte im Insolvenzverfahren - wie z.B. des Stimmrechts und des Widerspruchsrechts - und deren Ausübungsmöglichkeit zur Wahrung und Durchsetzung von den Interessen des Einzelnen in Bezug auf die übrigen Verfahrensbeteiligten. Wie und wann welches Teilnahmerecht im Verlauf des Anmelde- und Feststellungsverfahrens vom Insolvenzgläubiger begründet und mit welchen Auswirkungen diese im jeweiligen Verhältnis zu den Teilnahmerechten der übrigen Verfahrensbeteiligten ausgeübt werden können, soll im Verlauf dieser Arbeit untersucht und geklärt werden.

Diese Verfahrensinteressen und -rechte der Insolvenzgläubiger sind dabei den Interessen des Schuldners, eine möglichst hohe und nur berechtigte Gläubigerbefriedigung (Quote) bzw. bei Antrag auf Restschuldbefreiung eine weitgehende Enthaftung ${ }^{24}$ für einen wirtschaftlichen Neuanfang zu erreichen, ${ }^{25}$ gegenüberzustellen und je nach Verfahrenssituation untereinander in Einklang zu bringen. Diesbezüglich wird natürlich auch auf die Rolle des Insolvenzverwalters im Anmelde- und Feststellungsverfahren der $\S \S 179 f f$. InsO hinsichtlich seiner Aufgaben und

BGH ZinsO 2005, 597 (598) m.w.N.; siehe auch Pape, NZI 2004, 1 (3). Festgestellte Insolvenzforderungen aus vorsätzlich begangener unerlaubter Handlung sind gem. § 302 Nr.1 InsO von der Restschuldbefreiung ausgeschlossen.

25 Auf das Schuldnerinteresse hinsichtlich der Sanierung seines Unternehmens im Rahmen eines Insolvenzplans, soll in dieser Arbeit nicht weiter/vertiefend eingegangen werden. 
Interessenvertretung in Bezug $\mathrm{zu}$ den Insolvenzgläubigern und dem Schuldner einzugehen sein. 


\section{$\underline{\text { C. Teilnahmevoraussetzungen für das Verfahren }}$}

Zunächst soll (I.) eine differenzierende Erörterung des Insolvenzgläubigerbegriffs im Sinne des $\S 38$ InsO und der sich daraus ergebenden Betroffenenstellung als Grundvoraussetzung für eine Teilnahme am Anmelde- und Feststellungsverfahren der $\S \S 174 \mathrm{ff}$. InsO erfolgen. Danach soll (II.) genauer auf die einzelnen Tatbestandsmerkmale für die Qualifizierung einer Forderung als Insolvenzforderung und (III.) grundlegend auf die Stellung der nachrangigen Insolvenzgläubiger gem. § 39 InsO im Insolvenzverfahren eingegangen werden.

Diese Darlegungen sollen die Grundlage für die weiteren Ausführungen in Abschnitt D. bilden, um dort auf die einzelnen Voraussetzungen der Insolvenzforderungsanmeldung gem. $§ 174$ InsO und den diesbezüglich aufzuwerfenden Problematiken und Folgen eingehen zu können.

\section{Die Insolvenzforderung und ihr Gläubiger i.S.d. § 174 I 1 InsO}

Teilnahmevoraussetzung für das Anmeldeverfahren ist gem. § 174 I 1 InsO die individuelle Insolvenzgläubigerstellung. § $38 \mathrm{InsO}^{26}$ definiert diesen zentralen Begriff des Insolvenzgläubigers als einen persönlichen Gläubiger des Schuldners, dem zum Zeitpunkt der Verfahrenseröffnung ein Vermögensanspruch gegenüber dem Schuldner zusteht, und legt gleichzeitig fest, dass die Insolvenzmasse der gemeinschaftlichen Befriedigung aller Forderungen der Insolvenzgläubiger zu dienen hat. ${ }^{27}$ $\S 38$ InsO ist damit Ausfluss des in $\S 1$ Satz 1 InsO formulierten Ziels des Insolvenzverfahrens, der gemeinschaftlichen Befriedigung der Gläubiger, und konkretisiert es in zwei Richtungen.

Zum einen wird der Begriff der „Insolvenzforderung“ als ein zum Zeitpunkt der Verfahrenseröffnung begründeter persönlicher Vermögensanspruch gegen den Schuldner normiert. Zum anderen wird gleichzeitig der Zweck der Insolvenzmasse, deren Umfang durch die

Entspricht § 3 I KO und § 25 I VglO.

Aus dieser haftungsrechtlichen Zuweisung können die einzelnen Gläubiger jedoch kein dingliches Recht an Gegenständen der Insolvenzmasse herleiten. - Hess, InsO, $\S 38$ Rn.3; schon zur Konkursordnung siehe nur Jaeger/Henckel, KO, § 3 Rn.3; Hess/Hess, KO, § 3 Rn.1. 
$\S \S 35$ - 37 InsO festgelegt wird, durch ihre haftungsrechtliche Zuweisung an alle Gläubiger bestimmt. ${ }^{28}$ Konsequenz dieser Zuweisung ist, dass den Gläubigern in der Insolvenz des Schuldners die individuelle Rechtsverfolgung - wie sie außerhalb der Insolvenz zulässig ist - verwehrt ist (§ 89 InsO) und durch ein besonderes Verfahren ersetzt wird, das die Befriedigung aller Gläubiger - auf Kosten individueller Interessen - zum Ziel hat. ${ }^{29}$ Das persönliche Insolvenzgläubigerrecht ist dadurch charakterisiert, dass der Schuldner mit seinem ganzen Vermögen oder einem haftungsrechtlich relevanten Sondervermögen (vgl. § 333 InsO) im Ganzen für die Verbindlichkeiten einzustehen hat, so dass der Insolvenzgläubiger mit allen anderen Insolvenzgläubigern, die aus dem haftenden Vermögen Befriedigung suchen dürfen, konkurriert.

Vom persönlichen Insolvenzgläubigerrecht ist das dingliche spezielle Haftungsrecht zu unterscheiden, welches die Belastung eines bestimmten Gegenstandes des Vermögens für Forderungen des Schuldners beschreibt (§§ 47ff. InsO). Das dingliche Haftungsrecht bildet einen Anspruch auf vorzugsweise Sonderbefriedigung aus bestimmten Haftungsgegenständen, das dazu führt, dass der belastete Gegenstand nicht zur gemeinschaftlichen Befriedigung der übrigen Insolvenzgläubiger zur Verfügung steht, soweit der Verwertungserlös dem Inhaber des Haftungsrechts gebührt. ${ }^{30}$ Denn solche Aus- und Ersatzaussonderungsrechte ( $\S \S 47,48 \mathrm{InsO})^{31}$ sind ebenso wie Massenverbindlichkeiten ( $\S \S 53$ bis 55 InsO) ${ }^{32}$ gerade keine Insolvenzforderungen, ${ }^{33}$ und werden außerhalb des Insolvenzverfahrens gesondert befriedigt. Auch Absonderungsrechte ( $\S \S 49$ bis 51 InsO) als

Smid/Smid, § 38 Rn.1; MüKo-InsO/Ehricke, § 38 Rn.1; Kübler/Prütting/Bork/Holzer, $\S 38 \mathrm{Rn} .1$.

29 MüKo-InsO/Ehricke, § 38 Rn.1 m.w.N. Gleichzeitig geht das Recht des insolventen Schuldners, das zur Insolvenzmasse gehörende Vermögen zu verwalten und über es zu verfügen, auf den Insolvenzverwalter über (§ 80 I InsO).

30 RGZ 155, 95 (98f.); MüKo-InsO/Ehricke, § 38 Rn.10; Smid/Smid, InsO, § 38 Rn.8; Jaeger/Henckel, InsO, § 38 Rn.19; Nerlich/Römermann/Andres, § 38 Rn.3.

31 Den Aussonderungsberechtigten stehen jene gleich, die eine durch Vormerkung (§ 106 InsO) oder Anwartschaftsrecht (§ 107 I InsO) gesicherte Rechtsposition erlangt haben. - Siehe nur Gottwald/Klopp/Kluth, § 19 Rn.8 m.w.N.

32 Massegläubiger sind gem. § 53 InsO vorweg und damit außerhalb des Anmelde- und Feststellungsverfahrens der $\S \S 174 f f$. InsO zu befriedigen.

33 Gleichwohl müssen auch die Massegläubiger und Aus-/Absonderungsberechtigten ihre Ansprüche gem. § 28 II InsO zwecks Verzeichniserstellung (§§ 151, 152 InsO) beim Insolvenzverwalter anmelden. 
solche lösen keine derartigen Forderungen aus, ${ }^{34}$ es sei denn, der Schuldner haftet dem Absonderungsberechtigten neben dem Absonderungsgegenstand, der zur Insolvenzmasse gehören muss, auch persönlich; dann ist der Berechtigte zugleich vollwertiger Insolvenzgläubiger (§ 52 Satz 1 InsO). ${ }^{35}$

§ 38 InsO dient damit der Unterscheidung zwischen Insolvenzgläubigern und sonstigen Gläubigern, und beantwortet die Frage, welche der Gläubiger aufgrund ihres materiellrechtlichen Anspruchs gegen den Schuldner an der gemeinschaftlichen Befriedigung teilhaben dürfen und welche davon ausgeschlossen sind. ${ }^{36}$ Damit wird zugleich auch eine Aussage darüber getroffen, welche Gläubiger von den Folgen des Insolvenzverfahrens betroffen sind und den Beschränkungen - die für alle Insolvenzgläubiger gelten - unterfallen, auch wenn sie nicht am Verfahren teilnehmen. Denn zur Verfahrensteilnahme bedarf es stets der Anmeldung gem. § 174 InsO. Eine automatische direkte Verfahrensteilnahme erfolgt durch $\S 38$ InsO gerade nicht. ${ }^{37}$ Die Insolvenzgläubigerstellung knüpft daher zunächst an den materiellrechtlichen Anspruch des Gläubigers gegen den Schuldner an und nicht an die hieraus erwachsenen verfahrensrechtlichen Möglichkeiten (§§ 174, 178 InsO) oder teilnahmeunabhängigen Folgen (§ 301 I InsO). Insofern ist die Definition des Insolvenzgläubigers gem. § 38 InsO weniger verfahrensrechtlicher, sondern materiellrechtlicher Natur. ${ }^{38}$ Jedoch wird diese materiellrechtliche Einordnung der Definition in $\S 38$ InsO als Ausgangspunkt für eine notwendige Differenzierung hinsichtlich des Bestehens einer formellen Insolvenzgläubigerstellung zu hinterfragen sein:

$\S 38$ InsO enthält aufgrund der einheitlichen Zuweisung der Insolvenzmasse an alle Insolvenzgläubiger die qualitative Aussage, dass die Befriedigung der Insolvenzgläubiger durch das spezifisch

34 Absonderungsberechtigte besitzen ein zur vollen Befriedigung berechtigtes Vorzugsrecht am Erlös einzelner, haftungsrechtlich im Übrigen der Masse zugeordneter Gegenstände. - BGH ZIP 1996, 1307 (1307), Häsemeyer, InsO, Rn.18.03f.; Smid/Smid, § 38 Rn.8; KS/Eckardt, S.745 Rn.5.

35 Graf-Schlicker/Kalkmann, § 38 Rn.14; FK/Joneleit/Imberger, $\S 52 \quad$ Rn.2; Jaeger/Gerhardt, InsO, § 52 Rn.4f. m.w.N.

36 Häsemeyer, InsO, Rn.16.01; MüKo-InsO/Ehricke, § 38 Rn.2;

37 Dazu unter D.II. (S.30ff.).

38 Siehe auch Darstellung im Folgenden unter C.II. (S.16ff.). - BGH NZI 2005, 31 (31); MüKo-InsO/Ehricke, § 38 Rn.5; Kübler/Prütting/Bork/Holzer, § 38 Rn.1; GrafSchlicker/Kalkmann, § 38 Rn.1; Jaeger/Henckel, InsO, § 38 Rn.8; Jaeger/Henckel, KO, § 3 Rn.6f. 
insolvenzrechtliche Gerechtigkeitspostulat des Gläubigergleichbehandlungsgrundsatzes (,par condicio creditorum“) ${ }^{39}$ und durch die Gläubigerautonomie $^{40}$ geprägt ist. Diese beiden wichtigsten Grundsätze wirken sich im Insolvenzverfahren nicht nur durch Verfahrensrechte in Form des Teilnahme-, Widerspruchs- und Stimmrechts aus, sondern auch auf die persönlichen Forderungen der einzelnen Insolvenzgläubiger. Denn reicht das Schuldnervermögen, wie in der Insolvenz, nicht zur vollständigen Befriedigung aller angemeldeten Insolvenzforderungen aus, bedeutet eine gemeinschaftliche Befriedigung nicht nur eine gemeinsame, sondern immer auch eine anteilige Befriedigung. Ihre ursprünglichen Forderungen werden also nicht mehr voll, sondern nur zu einem bestimmten Prozentsatz (Quote) erfüllt, dessen Höhe davon abhängt, wie viel verwertbares Vermögen zur Verfügung steht. Diese für alle Insolvenzgläubiger geltende Quote steht für die gleichmäßige Befriedigung des einzelnen Insolvenzgläubigers im Verhältnis $\mathrm{zu}$ den übrigen Insolvenzgläubigern. ${ }^{41}$ Zugleich ergibt sich hieraus die grundsätzliche Gleichrangigkeit sämtlicher Insolvenzgläubiger, ${ }^{42}$ die nur ausnahmsweise und gesetzlich normiert durch die Nachrangigkeit minder schutzwürdiger Insolvenzforderungen (§ 39 InsO) oder abweichender Verteilungsmaßstäbe (§ 209 InsO) durchbrochen ${ }^{43}$ wird. ${ }^{44}$ Dabei müssen sich die Gläubiger ihren wechselseitigen Einfluss auf die Vermögens- und Haftungsverhältnisse des

39 Der Gleichbehandlungsgrundsatz liegt insbesondere den $\S \S 88,92,93,96,130-132$ und 195 InsO zugrunde. - Uhlenbruck/Uhlenbruck, § 38 Rn.1; Häsemeyer, InsO, Rn. 2.17ff.; HK/Kirchhof, § 1 Rn.4.

40 MüKo-InsO/Ganter, § 1 Rn.53; Uhlenbruck/Uhlenbruck, § 1 Rn.13; Hess, InsO, Vor $\S 1$ Rn.263ff. m.w.N.

41 „Par condicio“ - Häsemeyer, Rn.2.17ff., 16.20; Jaeger/Henckel, InsO, § 1 Rn.6; HK/Kirchhof, § 1 Rn.4; MüKo-InsO/Ehricke, § 38 Rn.4 und Ganter, § 1 Rn.51ff.; KS/Eckardt, S.744 Rn.2.

42 Die früheren Konkursvorrechte wurden durch den Gesetzgeber somit grundsätzlich abgeschafft.

43 Eine weitere Durchbrechung sieht § 32 DepotG vor. Siehe dazu Uhlenbruck/Uhlenbruck, § 38 Rn.1; Bauer, DZWir 2007, 188 (191).

44 Neben dem Gerechtigkeitsprinzip formaler Gleichheit steht das Differenzierungsgebot als widerstreitendes Element der Gerechtigkeit und die differenzierende Gläubigergleichbehandlung - etwa hinsichtlich der dinglich gesicherten Gläubiger gegenüber Insolvenzgläubigern - und beides sind gerade auch ein Gebot des Gleichheitssatzes. - Baur/Stürner, Insolvenzrecht, Rn.5.37; Carl, Teilnahmerechte, S.23. 
Schuldners als allseitigen Haftungsausgleich zurechnen lassen. ${ }^{45}$ Dieser durch die Möglichkeit der gegenseitigen Einflussnahme zum Ausdruck kommender Gläubigergleichbehandlungsgrundsatz innerhalb der Gläubigerautonomie im Insolvenzverfahren kann aber auch nur zwischen Insolvenzgläubigern derselben Verfahrensstellung gelten. Denn Gleichbehandlung innerhalb des Insolvenzverfahrens kann hinsichtlich der Ausübung von Verfahrensrechten nur bedeuten, dass die Einflussnahme des einzelnen Insolvenzgläubigers auf die Verfahrensrechte der übrigen Verfahrensteilnehmer immer nur so weit reicht, wie sich der Insolvenzgläubiger selbst der Einflussnahme auf seine eigene Verfahrensstellung durch seine Teilnahme am Verfahren aussetzt („Gleich unter Gleichen“). Dies spiegelt sich auch in der Systematik des ausnormierten Anmelde- und Feststellungsverfahrens wieder, welches ausschließlich an die ordnungsgemäß erfolgte Anmeldung der Insolvenzforderung gem. § 174 InsO anknüpft. So bezieht sich der Wortlaut der nachfolgend geregelten Eintragung, Prüfung und Feststellung von Insolvenzforderungen innerhalb der §§ 175, 176, 178 InsO auch nur auf angemeldete Insolvenzforderungen. Zudem wird durch § 177 InsO ausdrücklich die Verfahrensweise zur Einbindung von erst nachträglich erfolgten Anmeldungen in das Insolvenzverfahren geregelt, so dass innerhalb des Insolvenzverfahrens grundsätzlich zwischen angemeldeten und unangemeldeten Insolvenzforderungen zu unterscheiden ist. Diese Systematik und der Grundsatz der Gläubigergleichbehandlung haben zur Folge, dass die an die Anmeldung gebundenen Teilnahmerechte nur denjenigen Insolvenzgläubigern zustehen können, die (zumindest) eine ihrer Insolvenzforderungen zum Insolvenzverfahren angemeldet haben.

Insofern bleibt festzuhalten, dass bezüglich der Begründung von Verfahrensrechten im Insolvenzverfahren zwischen der Stellung eines rein materiellrechtlichen Insolvenzgläubigers, der - aus welchen Gründen auch immer - seine Insolvenzforderungen (noch) nicht zum Verfahren angemeldet hat, und der Stellung des formellen Insolvenzgläubigers, also

45 MüKo-InsO/Ehricke, § 38 Rn.4 und Ganter, § 1 Rn.52; Häsemeyer, InsO, Rn.2.17ff., 16.20; KS/Eckardt, S.744 Rn.2; Carl, Teilnahmerechte, S.59.

Durch diese Einbindung in eine „Haftungsgemeinschaft“, die meist eine Verlustgemeinschaft ist, werden jedoch keinerlei Rechtsbeziehungen der Insolvenzgläubiger untereinander begründet. - Uhlenbruck/Uhlenbruck, § 38 Rn.1; KS/Eckhardt, S.745 Rn.2 m.w.N. 
demjenigen der seine Insolvenzforderungen im Verfahren gem. § 174 InsO ordnungsgemäß angemeldet hat, zu differenzieren ist. Grundsätzlich unterliegen aber beide, wegen ihrer Grundeigenschaft als Insolvenzgläubiger im Sinne des $\S 38$ InsO, den gesetzlichen Beschränkungen und den sonstigen Rechtsfolgen der Insolvenzordnung (z.B. §§ 89, 301 I InsO). Dies folgt aus $\S 1$ S.2 InsO, wonach als weiteres Ziel des Insolvenzverfahrens dem redlichen Schuldner die Gelegenheit gegeben werden soll, sich von seinen bisherigen Verbindlichkeiten befreien zu können. Würden daher nicht sämtliche Insolvenzgläubiger von den Rechtsfolgeregelungen betroffen sein, würde dieses Ziel nicht erreicht werden können.

Im weiteren Verlauf der Arbeit werden die Bedeutung und Notwendigkeit, zwischen diesen Verfahrensstellungen zu differenzieren, für die Beurteilung und Durchführung eines einheitlichen Insolvenzverfahrens hinsichtlich der Begründung und interessensgerechten Gewährung von Verfahrensrechten im Einzelnen ersichtlich werden, insbesondere hinsichtlich der Verfahrensrechte von nachrangigen Insolvenzgläubigern und im Rahmen der Akteneinsicht. ${ }^{46}$

46 Siehe ab D.VI.1. und 3. (S.94ff., 108ff.). 


\section{Voraussetzungen für eine Qualifikation als Insolvenzforderung}

\section{Vermögensanspruch gegen den Schuldner}

Durch das Wesen des Insolvenzverfahrens als einer auf die gleichmäßige Befriedigung der Gläubiger in Geld zielende Gesamtvollstreckung knüpft $\S 38$ InsO die Insolvenzgläubigereigenschaft qualitativ an das Bestehen eines Vermögensanspruchs gegen den Schuldner an. Nicht vermögensrechtliche Ansprüche, wie z.B. höchstpersönliche Leistungen

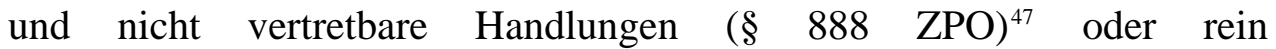
familienrechtliche Ansprüche, sind keine Insolvenzforderungen und damit grundsätzlich von einer Teilnahme am Verfahren ausgeschlossen, ${ }^{48}$ weil das Insolvenzverfahren ausschließlich der Realisierung der vermögensrechtlichen Haftung des Schuldners bzw. des Schuldnerunternehmens dient. Es muss sich also um eine Forderung handeln, die eine Geldleistungspflicht zum Gegenstand hat oder zwar nicht auf Geldzahlung gerichtet ist, sich aber inhaltlich nach Maßgabe der $\S \S 45$, $46 \mathrm{InsO}^{49}$ in einen Geldleistungsanspruch in Euro umwandeln lässt. ${ }^{50}$ Diese Umwandlung hat zunächst verfahrensrechtliche Bedeutung, um Insolvenzforderungen, die nicht auf Geld gerichtet sind oder deren Geldbetrag unbestimmt ist, die Teilnahme am Insolvenzverfahren und deren Vergleichbarkeit mit den übrigen Insolvenzforderungen zu ermöglichen. ${ }^{51}$

Zudem kommt es für die Einordnung des Anspruchs als Insolvenzforderung entgegen dem gesetzlichen Terminus „persönlicher Anspruch“ nicht darauf an, welche Rechtsgrundlage der Anspruch hat. ${ }^{52}$ Vielmehr kann sich die persönliche Haftung des Schuldners unmittelbar aus dem Gesetz, aus Vertrag, ungerechtfertigter Bereicherung oder aus

\footnotetext{
47 Zur insolvenzspezifischen Rechtssprechung des $\S \quad 888$ ZPO siehe etwa FK/Schumacher, § 38 Rn.9.

48 Kübler/Prütting/Bork/Holzer, § 38 Rn.16f.; Hess, InsO, § 38 Rn.37; Smid/Smid, $\S 38$ Rn.10, 16; FK/Schumacher, § 38 Rn.9; KS/Eckardt, S.746 Rn.6.

49 Entspricht den vormaligen $\S \S 69,70 \mathrm{KO}$; §§ 34, $35 \mathrm{VglO}$.

50 Kübler/Prütting/Bork/Holzer, $\S 38$ Rn.13; FK/Schumacher, $\S \quad 38$ Rn.8; Jaeger/Henckel, KO, § 3 Rn.20; Hess, InsO, § 38 Rn.13 m.w.N.

51 Begr. zu §§ 52, 53 (§§ 45, 46 InsO) RegE, BT-Drucks. 12/2443, S.124; BGHZ 113, 207 (214).

52 Hess, InsO, § 38 Rn.31; FK/Schumacher, § 38 Rn.9; Gottwald/Klopp/Kluth, § 19 Rn.8; Haarmeyer/ Wutzke/Förster, Kap.7 Rn.1.
} 
einer Verfügung von Todes wegen ergeben. ${ }^{53}$ Der Zweck dieser Formulierung liegt nämlich nicht (nur) darin, formal die Gläubiger von den Nichtgläubigern abzugrenzen. ${ }^{54}$ Sondern es wird dahingehend differenziert, ob die Forderung des Gläubigers gegen den Schuldner das allgemeine, mit den Befriedigungsrechten der anderen Gläubigern konkurrierende Teilhaberecht am Gesamtvermögen des Schuldners (Insolvenzmasse) begründet, oder ein diesen gegenüber vorrangiges Recht auf ausschließliche Befriedigung an einzelnen in der Masse befindlichen Gegenständen (§§ 47ff. InsO). ${ }^{55}$

\section{Zeitpunkt der Anspruchsbegründung}

Insolvenzforderungen setzen weiterhin voraus, dass sie zur Zeit der Eröffnung des Verfahrens begründet, d.h. vor Eröffnung entstanden sind. ${ }^{56}$ Entstehende Abgrenzungsfragen werden durch die gesetzlichen Regelungen der $\S \S 41,42,191$ InsO größtenteils beantwortet. Aus diesen Regelungen lässt sich der Grundsatz ableiten, dass bei Verfahrenseröffnung die Forderung zumindest ihrem Rechtsgrund nach bzw. eine „gesicherte haftungsrechtliche Anwartschaft am Vermögen des Schuldners“ bestanden haben muss. ${ }^{57}$

Neugläubiger, also Gläubiger die ihre Forderungen gegen den Schuldner bzw. gegen die Insolvenzmasse erst während des Insolvenzverfahrens erlangen, sind daher notwendig keine Insolvenzgläubiger und nehmen grundsätzlich nicht am Verfahren der §§ 174ff. InsO teil. Gleichwohl ist auch bei der Begründung von Verbindlichkeiten gegenüber Neugläubigern hinsichtlich ihrer Befriedigungsmöglichkeit danach abzugrenzen, von wem

Uhlenbruck/Uhlenbruck, $\S 38$ Rn.5; Kuhn/Uhlenbruck, $\S 3$ KO Rn.10; Kübler/Prütting/Bork/Holzer, § 38 Rn.14ff.; Hess, InsO, § 38 Rn.31ff. jeweils mit weiteren Beispielen.

54 So aber wohl Kübler/Prütting/Bork/Holzer, § 38 Rn.1.

55 MüKo-InsO/Ehricke, $\S 38$ Rn.10ff.; Jaeger/Henckel, InsO, $\S 38$ Rn.8; Gottwals/Klopp/Kluth, § 19 Rn.8; Haarmeyer/Wutzke/Förster, Kap.7 Rn.1; Hess, InsO, § 38 Rn.5; KS/Eckardt; S.745 Rn.4; Jaeger/Henckel, KO, § 3 Rn.6.

56 Diese Abgrenzung ist nur sachgerecht, wenn der Schuldner mit Verfahrenseröffnung seine Verfügungsbefugnis über die Insolvenzmasse verliert ( $\S \S 80 \mathrm{ff}$. InsO) und damit korrespondierend auch seine Verpflichtungsbefugnis zulasten der Insolvenzmasse.

57 BGH NZI 2005, 403 (403); BAG NJW 1979, 774 (774); BFH ZIP 1994, 1286 (1286f.); HK/Eickmann, § 38 Rn.16; Graf-Schlicker/Kalkmann, § 38 Rn.15; siehe im Einzelnen ausführlich bei MüKo-InsO/Nowak, § 174 Rn.18. 
diese Verbindlichkeiten eingegangen worden sind. Geht der Schuldner nach Verfahrenseröffnung neue Verbindlichkeiten ein, können die Neugläubiger, aufgrund der Zuweisung der Masse an die Insolvenzgläubiger durch $\S 38$ InsO, nur Befriedigung aus dem insolvenzfreien Vermögen des Schuldners erlangen. ${ }^{58}$ Sollten die Verbindlichkeiten hingegen von einem Insolvenzverwalter oder vom Schuldner im Rahmen der Eigenverwaltung begründet worden sein, sind diese Gläubiger keine Neugläubiger im eigentlichen Sinn, sondern gem. $\S 55$ InsO Massegläubiger und können mit ihren Forderungen als gesondert und vorweg $\mathrm{zu}$ befriedigenden Masseforderungen ( $\S 53$ InsO) am Insolvenzverfahren beteiligt sein.

Das tatsächliche Bestehen der angemeldeten Forderung ist in diesem Verfahrensabschnitt nicht Voraussetzung für die Stellung des angeblichen oder tatsächlichen Berechtigten als Insolvenzgläubiger. Für die Anmeldung zur Teilnahme am Insolvenzverfahren genügt die Glaubhaftmachung einer Forderung, die, ihr Bestehen unterstellt, die Voraussetzungen einer Insolvenzforderung erfüllt. ${ }^{59}$ Das Bestehen oder Nichtbestehen der angemeldeten Insolvenzforderung ist Frage des Prüfungstermins, wonach nur gemeinschaftlich über die Berechtigung zur weiteren Teilnahme am Verfahren zu entscheiden ist. ${ }^{60}$ Hierdurch wird zwar einerseits ein zügiges Anmeldeverfahren gewährleistet, indem nur eine Prüfung hinsichtlich der formellen Voraussetzungen der Anmeldung für eine Aufnahme der Insolvenzforderung in die Insolvenztabelle vom Insolvenzverwalter durchgeführt wird. Andererseits wird durch die „lediglich“ formell ordnungsgemäße Anmeldung bereits die Teilnahme am weiteren Feststellungsverfahren und die damit verbundenen weitreichenden Teilnahmerechte begründet, ${ }^{61}$ sodass die Hürde für eine bewusst unberechtigte bzw. rechtsmissbräuchliche Einflussmöglichkeit auf das weitere Verfahren durch Dritte in diesem Verfahrensabschnitt recht gering ist. Wie sich im Verlauf der Arbeit zeigen wird, stehen den

Siehe dazu auch unter E.III.2. (S.208ff.). - Uhlenbruck/Uhlenbruck, § 38 Rn.1; HK/Eickmann, § 38 Rn.16; Häsemeyer, InsO, Rn.10.04f.

59 Smid/Smid, § 38 Rn.13; Nerlich/Römermann/Andres, § 38 Rn.13; MüKoInsO/Ehricke, § 38 Rn15.; Jaeger/Henckel, InsO, § 38 Rn.9; Hess, InsO, § 38 Rn.14; Haarmeyer/Wutzke/Förster, Kap.7 Rn.14; Häsemeyer, InsO, Rn.16.11ff.; Jaeger/Henckel, KO, § 3 Rn.6f.

60 Siehe dazu unter D.IV.5.a) (S.53ff.) und E.I.3. (S.137ff.).

61 Siehe unter D.VI.1. (S.94ff.). 
Verfahrensteilnehmern jedoch weitreichende Möglichkeiten durch Widerspruchserhebung (§ 176 InsO) und Stimmrechtsfeststellung (§ 77 II InsO) zur Verfügung, um einer „echten“ Einflussnahme gegen Unberechtigte entgegentreten zu können. 


\section{Nachrangige Insolvenzforderungen}

$\S 38$ InsO definiert den Begriff des Insolvenzgläubigers materiellrechtlich und ordnet die Gleichbehandlung der Insolvenzgläubiger an. ${ }^{62}$ Durch $§ 39$ InsO besteht nunmehr zumindest die Möglichkeit auch die dort aufgeführten Forderungen im Insolvenzverfahren geltend $\mathrm{zu}$ machen, ${ }^{63}$ jedoch wird der Gleichbehandlungsgrundsatz hinsichtlich der Gläubiger dieser Forderungen zugunsten eines zügigen Verfahrensfortgangs eingeschränkt. Zwar sind auch die in $\S 39$ InsO aufgeführten Insolvenzforderungen nach der Definition Insolvenzforderungen, allerdings wird deren Inhabern durch diese Vorschrift der Nachrang zu den sonstigen Insolvenzgläubigern zugewiesen und die Befriedigungsreihenfolge untereinander geordnet. ${ }^{64}$ Nachrangige Insolvenzforderungen sind somit zunächst die in den fünf Rangklassen des § 39 I InsO genannten Ansprüche, nach diesen sodann die Forderungen, deren Nachrang vereinbart worden sind (§ 39 II InsO), und schließlich im Nachlassinsolvenzverfahren die Forderungen der beiden Rangklassen gem. $\S 327$ I InsO. $^{65}$

Aussicht auf Befriedigung aus der Insolvenzmasse haben nachrangige Gläubiger allerdings bloß dann, wenn (1) das Insolvenzgericht zu deren Anmeldung und Prüfung gesondert aufgefordert hat (§§ 174 III 1, 177 II InsO) oder (2) ein Insolvenzplan vorgelegt wird, der Zahlungen an die nachrangigen Gläubiger vorsieht. ${ }^{66}$ Eine Aufforderung zur Anmeldung wird nur dann ergehen, wenn die vorrangigen Gläubiger voll befriedigt werden konnten und darüber hinaus ein Überschuss verbleibt. Solch ein Überschuss wird jedoch meist erst im Verlauf des Verfahrens festgestellt, so dass die Aufforderung zur Anmeldung von nachrangigen Forderungen,

62 Dazu bereits unter C.I. und II. (S.10ff., 16ff.).

63 Die Teilnahme von nachrangigen Gläubigern am Verfahren war vor der jetzigen Regelung in § 39 InsO nach § $63 \mathrm{KO}$, $29 \mathrm{VglO}$, § 32a I 1 GmbHG a.F. ausgeschlossen.

64 Die Einbeziehung nachrangiger Insolvenzgläubiger in das Insolvenzverfahren ermöglicht vor allem im Fall eines Insolvenzplans, die Rechtsstellung dieser Gläubiger sachgerecht zu bestimmen (vgl. §§ 225, 246 InsO). - Begr. zu § 46 (§ 39 InsO) RegE, BT-Drucks. 12/2443, S.123.

65 Einen zusätzlichen Anwendungsbereich erhält der Forderungsnachrang z.B. über die $\S \S 264,266$ InsO. Zur Einordnung einer Forderung als nachrangige Forderung siehe etwa die Übersicht bei Hess, InsO, § 174 nach Rn.117.

66 Die Insolvenzverfahren, in denen keine dieser Ausnahmen vorliegt, sollen mit der Anmeldung und Prüfung nachrangiger Forderungen nicht belastet werden. - Begr. zu § 201 (§ 174 InsO) RegE, BT-Drucks. 12/2443, S.184. 
wenn überhaupt, regelmäßig nachträglich und mit den Folgen eines besonderen Prüfungstermins oder eines schriftlichen Verfahrens (§ 177 II InsO) ergehen wird. ${ }^{67}$

Weiterhin gelten für nachrangige Insolvenzgläubiger, soweit keine abweichenden Regelungen normiert sind, dieselben Regelungen wie für die nicht-nachrangigen Insolvenzgläubiger. ${ }^{68}$ Abweichend sind nachrangige Insolvenzgläubiger beispielsweise grundsätzlich nicht stimmberechtigt ( $§ 77$ I 2 InsO), ${ }^{69}$ auch nicht bei Abstimmungen über einen Insolvenzplan (vgl. § 246 InsO). Weiterhin sind ihre Forderungen bei Abschlagsverteilungen nicht zu berücksichtigen (§ 187 II 2 InsO) und gelten im Insolvenzplan als im Zweifel erlassen (§ 225 I InsO).

Diese Abweichungen in Verbindung mit der gleichzeitigen Geltung des Vollstreckungsverbots ( $\S 89$ InsO) und der Erfassung von einer eventuellen späteren Restschuldbefreiung („gegenüber allen Insolvenzgläubigern“, $§ 301$ I InsO) ${ }^{70}$ sollen sicherstellen, dass das Insolvenzverfahren durch die Einbeziehung der nachrangigen Insolvenzgläubiger in der Großzahl der Fälle, in denen diese ohnehin keine Aussicht auf Befriedigung haben, nicht nachteilig verzögert wird. ${ }^{71}$ Nachrangige Insolvenzgläubiger haben in der Praxis somit nur eine äußerst geringe Befriedigungschance.

67 Begr. zu $\quad \S \quad 201 \quad$ (§ $174 \quad$ InsO) RegE, BT-Drucks. 12/2443, S.184;

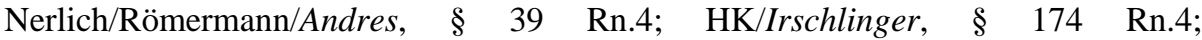
Uhlenbruck/Uhlenbruck, § 39 Rn.1; Hess/Weis/Wienberg/Weis, § 174 Rn.69. Siehe auch unter E.II.6. (S.203ff.).

Begr. zu § 46 (§ 39 InsO) RegE, BT-Drucks. 12/2443, S.123; HK/Eickmann, § 39 Rn.6; Jaeger/Henckel, InsO, § 39 Rn.8.

69 Siehe außerdem $\S \S 75,78$ I InsO.

70 Mit der Ausnahme gem. § 302 Nr.2 InsO für Gläubiger der in § 39 I Nr.3 InsO aufgeführten Geldstrafen und gleichgestellten Verbindlichkeiten.

71 Begr. zu § 46 (§ 39 InsO) RegE, BT-Drucks. 12/2443, S.123. 


\section{Zwischenergebnis}

Für die weiteren Untersuchungen des Anmelde- und Feststellungsverfahrens bleibt damit zunächst festzuhalten, dass hinsichtlich der Begründung und Ausübung von Verfahrensrechten zwischen der materiellrechtlichen und der verfahrensrechtlichen Insolvenzgläubigerstellung zu unterscheiden sein wird. Dies gilt grundsätzlich auch für nachrangige Insolvenzgläubiger, soweit keine abweichenden Regelungen normiert sind. 


\section{Anmeldung und Tabelleneintragung der Insolvenzforderungen}

Im folgenden Abschnitt (I.) soll zunächst eine verfahrensrechtliche Einordnung des Insolvenzverfahrens erfolgen, um die eigenständigen Wesenszüge des Insolvenzverfahrens und deren Bedeutung hinsichtlich seiner verfahrensrechtlicher Bezüge zur Zivilprozessordnung im weiteren Verlauf der Bearbeitung des Anmelde- und Feststellungsverfahrens darzulegen. Danach sollen die Anforderungen an die Insolvenzforderungsanmeldung gem. § 174 InsO zur Teilnahme am Insolvenzverfahren erörtert und der grundsätzliche Verlauf und die entsprechende Durchführung des Anmeldeverfahrens aufgezeigt und untersucht werden, um schließlich auf die Begründung weitreichender Teilnahmerechte durch die ordnungsgemäß erfolgte Anmeldung einzugehen.

\section{Verfahrensrechtliche Einordnung des Insolvenzverfahrens}

Die grundsätzliche Aufgabe des Insolvenzverfahrens als Zivilverfahren ${ }^{72}$ besteht darin, durch Mitwirkung der Insolvenzgläubiger die persönliche Haftung eines in Vermögensverfall geratenen Schuldners oder dessen Sanierung unter Verlustgemeinschaft der Insolvenzgläubiger ${ }^{73}$ mit Hilfe der Staatsgewalt zu verwirklichen, ${ }^{74}$ und darüber hinaus dem redlichen Schuldner die Möglichkeit zur Schuldbefreiung zu geben (vgl. § 1 InsO).

Während die Konkursordnung in ihrem ersten Buch unter der Überschrift „Konkursrecht“ (§§ 1 bis 70 KO) und im zweiten Buch „Konkursverfahren“ ( $\S \S 71$ bis $238 \mathrm{KO}$ ) noch versuchte, das materielle und das formelle Konkursrecht $\mathrm{zu}$ trennen, folgt die Gliederung der Insolvenzordnung im Großen und Ganzen dem typischen Ablauf eines Insolvenzverfahrens. ${ }^{75}$ Gleichwohl lässt sich auch innerhalb der

\footnotetext{
Ergibt sich unstreitig aus den gesetzlichen Verweisungen (§§ 72 KO, 115 VerglO, 4 InsO). Vgl. auch Hess, InsO, Vor § 2 Rn.8.

73 Schlagwörter: Haftungszuweisung - Gläubigergleichbehandlungsgrundsatz Gläubigerautonomie; § 38 InsO. Siehe bereits unter B. (S.4ff.).

74 Häsemeyer, InsO, Rn.3.02; Jaeger/Gerhardt, InsO, § 2 Rn.7; MüKo-InsO/Ganter, Vor §§ 2-10 Rn.5f.

75 Dies wird den engen Wechselwirkungen zwischen formellem und materiellem Insolvenzrecht gerecht. Siehe dazu auch Häsemeyer, InsO, Rn.3.02; Uhlenbruck/Uhlenbruck, § 1 Rn.2.
} 
Insolvenzordnung differenzieren: Das Insolvenzverfahrensrecht setzt sich dabei aus den Vorschriften zusammen, die das Verfahren zur Durchsetzung der materiellrechtlichen Ansprüche der Insolvenzgläubiger vor dem Insolvenzgericht - flankiert von der Möglichkeit der Inanspruchnahme staatlicher Zwangseinwirkung - betreffen und zugleich die Ordnung des Verfahrensablaufes gewährleisten. ${ }^{76}$ Normen des materiellen Insolvenzrechts sind demgegenüber solche Rechtssätze, die eine Wirkung auf die materielle Rechtslage entfalten, also inhaltlich die Rechtsstellung der Beteiligten im zivilrechtlichen Anspruchssystem beeinflussen. ${ }^{77}$ Vielfach sind jedoch materiellrechtliche und verfahrensrechtliche Regelungen miteinander verbunden (vgl. z.B. §§ 122, 125ff., 245, 247 II, 248ff. InsO). ${ }^{78}$ Aufgrund dieser Verknüpfungen des materiellen und formellen Insolvenzrechts innerhalb der Insolvenzordnung und der damit einhergehenden Wechselwirkungen wird „das Insolvenzverfahrensrecht“ daher in der Regel als das Recht des Insolvenzverfahrens als Gesamtvollstreckungsverfahren bezeichnet. ${ }^{79}$

Dementsprechend lassen sich auch je nach Blickwinkel „Wesensverwandtschaften“ des Insolvenzverfahrens mit verschiedenen anderen prozessualen Verfahrensarten erkennen. Die wesensmäßige Einordnung des Insolvenzverfahrens in eine Verfahrensart (streitige oder freiwillige Gerichtsbarkeit) hängt davon ab, ob man innerhalb der für das Insolvenzverfahren eigentümlichen Mischung verschiedener verfahrensrechtlicher Elemente zu einem Überwiegen der einen oder anderen Elemente neigt und dies für die Bestimmung der Rechtsnatur des Verfahrens insgesamt für ausschlaggebend hält. ${ }^{80}$

76 Vorschriften des formellen Insolvenzrechts sind etwa die das Insolvenzgericht und die Zustellung und öffentliche Bekanntmachung betreffenden $\S \S 2-10$ InsO, die Regelung des Eröffnungsverfahrens ( $\S \S 13-34$ InsO) und die Feststellung der Insolvenzforderungen (§§ 174ff. InsO). - Gerhardt, Grundbegriffe, Rn.258; ferner Häsemeyer, InsO, Rn3.02; siehe eingehend zur Abgrenzung und Wechselwirkung von Zivilprozessrecht und materiellem Recht bei Henckel, Prozessrecht.

77 Hierzu gehören z.B. die Vorschriften über die Aus- und Absonderung ( $\S \S 47 f f . ~ I n s O)$, der Masseverbindlichkeiten und Massegläubiger (§§ 53ff. InsO) und der Anfechtung (§§ 129ff. InsO). - Gerhardt, Grundbegriffe, Rn.258.

78 Weitere Beispiele bei Uhlenbruck/Uhlenbruck, § 1 Rn.2; Jaeger/Gerhardt, InsO, $\S 2$ Rn.5.

79 Eine Trennung zwischen materiellem und formellem Insolvenzrecht ist so gut wie nicht mehr möglich. Nähere Darstellung bei Häsemeyer, InsO, Rn.3.06ff.; Baur/Stürner, Insolvenzrecht, Rn.1.11f.

80 Die Möglichkeit einer unterschiedlichen Auffassung ist schon in den Motiven zur Konkursordnung gesehen worden. - KO-Motive, S.293ff. = Hahn, KO, S.270ff. 


\section{Kein Erkenntnisverfahren}

In verfahrensrechtlicher Hinsicht ist das Insolvenzverfahren zumindest kein Erkenntnisverfahren, obwohl es teilweise als quasi-streitiges Parteiverfahren ausgestaltet ist, in dem sich Antragsteller (Gläubiger) und Antragsgegner (Schuldner) als Parteien gegenüberstehen, und der Verwirklichung der persönlichen Haftung eines insolventen Schuldners mit Hilfe der Staatsgewalt dient. ${ }^{81}$ Das Gericht fordert zwar öffentlich auf, die Gläubigerforderung anzumelden (§§ 28, 174ff. InsO), und beurkundet das Ergebnis der "Unbestrittenheit" der Insolvenzforderungen mit dem Erfolg der Rechtskraft und Vollstreckbarkeit (§§ 178 III, 201 II InsO), jedoch entscheidet es gerade nicht selbstständig über streitige Forderungen. Das Insolvenzgericht handelt nicht in erkennender Amtstätigkeit, wie etwa beim „Verurteilen“ gem. § 307 ZPO, sondern seine Tätigkeit erschöpft sich vielmehr in der Mitwirkung bei der Feststellung zur Tabelle durch Protokollierung (§ 178 II 1 InsO). ${ }^{82}$ Gleiches gilt für die Feststellung sonstiger Gläubigerrechte. Streitentscheidungen sind nämlich gerade nicht Sache des Insolvenzgerichts und Teil des Insolvenzverfahrens, sondern ausdrücklich im zivilen Prozessweg zu treffen (§£ 179ff. InsO) ${ }^{83}$ Insoweit wurden schon in den Motiven zur Konkursordnung hinsichtlich des Prüfungstermins u.a. die jetzigen $\S \S$ 286, 287 ZPO zur freien Beweisaufnahme und -würdigung ausdrücklich für nicht anwendbar erklärt. $^{84}$

81 Jaeger/Gerhardt, InsO, § 2 Rn.10; Uhlenbruck/Uhlenbruck, § 1 Rn.2; Baur/Stürner, Insolvenzrecht, Rn.1.11; Pape/Uhlenbruck, InsO, Rn.32, 95 m.w.N.

82 Siehe dazu auch D.IV.5.a) (S.53ff.).

83 Das Insolvenzrecht hat auf diesen Prozessweg nur insoweit mittelbaren Einfluss, als abweichend von den allgemeinen Bestimmungen das örtlich zuständige Prozessgericht immer das Amtsgericht - bei dem das Insolvenzverfahren anhängig ist oder anhängig war - bzw. das in dessen Bezirk liegende Landgericht ist ( $§ 180 \mathrm{I}$ InsO).

84 „Die Erörterung in dem [Prüfungs-] Termine ist eine mündliche, ohne daß die Grundsätze der Civilprozeßordnung über die Nothwendigkeit mündlichen Vorbringens von Parteianträgen und Behauptungen (§£ 124, 125, 259, 260 u.a.) zur Anwendung gelangen könnten. “ KO-Motive, S.361 = Hahn, KO, S.325; vergleiche zu den Normänderungen Seuffert, ZPO 1877, §§ 259, 260; ders., ZPO 1898, $\S \S 286,287$. 


\section{Aspekte der streitigen Gerichtsbarkeit}

Der Verfahrenszweck des Insolvenzverfahrens liegt letztlich in der Haftungsverwirklichung, indem das Vermögen des Schuldners beschlagnahmt und verwertet bzw. in einen Insolvenzplan eingebunden wird. Dass darüber hinaus dem redlichen Schuldner die Möglichkeit zur Restschuldbefreiung gegeben wird, ändert hieran nichts, da der Versuch einer möglichst weitreichenden Haftungsverwirklichung stets notwendige Vorstufe zur Restschuldbefreiung ist. Mit Blick auf dieses einleitende Verfahrensziel ähnelt das Insolvenzverfahren daher einem Vollstreckungsverfahren und steht damit grundsätzlich der streitigen Gerichtsbarkeit nahe. ${ }^{85}$ Dieser Einordnung trägt die Insolvenzordnung auch dadurch Rechnung, dass sie gem. § 4 InsO die entsprechende Anwendung der Vorschriften der Zivilprozessordnung vorschreibt, soweit nichts Gegenteiliges bestimmt ist. In der gleichzeitigen Erfassung des gesamten beschlagfähigen Schuldnervermögens zugunsten aller Insolvenzgläubiger und in deren grundsätzlicher Gleichbehandlung tritt das Insolvenzverfahren als Gesamtvollstreckungsverfahren selbstständig und diese verdrängend (§ 89 InsO) neben „die Zwangsvollstreckung“ im Sinne der $\S \S 704 f f$. ZPO. $^{86}$ Das Parteienverhältnis liegt wie bei der Einzelvollstreckung auch im Insolvenzverfahren im Gegenüber von Gläubiger und Schuldner, das sich in der möglichen zwangsweisen Durchsetzung der Haftungsverwirklichung zugunsten der Insolvenzgläubiger und deren Einwirkungsmöglichkeit auf das Verfahren durch ihre Mitwirkungsrechte ausdrückt, worin ein grundsätzlicher immanenter Interessenstreit besteht: Der Insolvenzgläubiger will hinsichtlich seiner materiellen Forderung in einem gerichtlichen (geführten) Verfahren sein „Recht“ - durch Feststellung - auf Teilhabe an der Insolvenzmasse zugesprochen bekommen, das grundsätzlich über den

Schon zur KO siehe RG LZ 1911, Sp.557; BGH NJW 1961, 2016 (2016f.); Jaeger/Weber, KO, Vor § 71 Rn.3; Kuhn/Uhlenbruck, § 72 Rn.1.

Herrschend auch zur InsO: BVerfG NZI 2006, 453 (454) Insolvenzverfahren als „Teil des Zwangsvollstreckungsrechts“; weiter Kübler/Prütting/Bork/Prütting, § 5 Rn.4; Hess, InsO, Vor § 2 Rn.2, 8f.; KS/Vallender, S.251 Rn.2; Uhlenbruck/Uhlenbruck, $\S 1$ Rn.2; KS/Prütting, S.221 Rn.1; Jaeger/Gerhardt, InsO, § 2 Rn.10, 19; Häsemeyer, InsO, Rn.1.11ff., 3.05; Jauernig, S.275; MüKo-InsO/Ganter, Vor §§ 2-10 Rn.5 m.w.N.

86 Siehe bereits unter B. (S.4ff.). 
Zeitraum des Insolvenzverfahrens hinaus wirkt ${ }^{87}$ und gleichzeitig seinen Einfluss auf das Verfahren sichert. Der Schuldner hingegen ist daran interessiert, dass keine unberechtigten Ansprüche gegen ihn durchgesetzt werden können und er nach Abschluss des Verfahrens weitreichend von seinen Schulden befreit wird (§§ 301, 302 InsO). Das gleiche Interesse besteht bei den Insolvenzgläubigern, da unberechtigte Ansprüche die (eigene) Quote verschlechtern.

\section{Aspekte der freiwilligen Gerichtsbarkeit}

Gleichzeitig weist das Insolvenzverfahren jedoch eigenständige Wesenzüge auf, durch die es sich von der Zwangsvollstreckung unterscheidet und der freiwilligen Gerichtsbarkeit annähert: ${ }^{88}$ Das Insolvenzverfahren kann auch auf Antrag des Schuldners - also ohne jede Initiative des Gläubigers - eröffnet werden (§ 13 I S.2). Ein Gläubiger bedarf, um die Eröffnung des Verfahrens zu beantragen und an dem Verfahren teilzunehmen, keines Vollstreckungstitels (§§ 14 I, 174ff. InsO). Die Insolvenzgläubiger haben weitgehende Gestaltungsbefugnisse etwa hinsichtlich eines Insolvenzplanes ( $\S \S 217 f f$. InsO), sogar die Eigenverwaltung und Verwertung durch den Schuldner wird ermöglicht (§§ 270ff. InsO). ${ }^{89}$

Aber: Die Tatsache, dass der Schuldner einen eigenen Antrag zur Eröffnung eines Insolvenzverfahrens stellen kann, macht diesen nicht immer zu einem „freiwilligen“, da etwa der Schuldner als juristische Person - also die Organe - unter Strafandrohung und Androhung zivilrechtlicher Sanktionen zur Stellung eines Insolvenzantrages verpflichtet sind (bezüglich einer GmbH gem. § 15a InsO; § 64 GmbHG). Dadurch, dass die Verfahrenseröffnung mit dem Verlust der Verwaltungsund Verfügungsbefugnis einhergeht (§ 80 InsO) und die Beschlagnahme

Siehe auch unter E.III.2. (S.208ff.).

Dies betonend: Smid/Smid, Einleitend vor § 1 Rn.16, § 4 Rn7; Hess/Pape/Pape, Rn.6; Nerlich/Römermann/Becker, § 2 Rn.8; weitergehend Münzel, ZZP 66 (1953), 334 (337f.); Bötticher, ZZP 86 (1973), 373 (378ff.). Siehe dazu Ausführungen bei Jaeger/Gerhardt, InsO, § 2 Rn.19ff.

89 Weitere Beispiele bei MüKo-InsO/Ganter, Vor $\S \S 2-10$ Rn.6ff. m.w.N. 
des Schuldnervermögens zur Folge hat (§ 35 InsO), ist die „Freiwilligkeit des Verfahrens“ nicht gegeben.

Auch dass der Gläubiger ohne vollstreckbaren Titel die Eröffnung des Insolvenzverfahrens beantragen kann, spricht ebenfalls nicht unbedingt für eine (ausschließliche) Zuordnung des Insolvenzverfahrens zur freiwilligen Gerichtsbarkeit. Vielmehr sind dem Gläubiger langwierige Prozessführungen bei akut drohender Insolvenz nicht zumutbar, weil dies die Zugriffsmöglichkeit auf das Schuldnervermögen verringert. ${ }^{90}$

Ebenso kommt eine Sanierung mittels des Insolvenzplans nicht ausschließlich den Schuldnerinteressen entgegen, vielmehr gestattet der Insolvenzplan, aufgrund der geringen Hürde der Unbeachtlichkeit eines Schuldnerwiderspruchs gegen den Plan (§ 247 II InsO), die vorrangige Berücksichtigung der Insolvenzgläubigerinteressen. Das Zurücktreten einzelner Gläubigerinteressen im Insolvenzplan zugunsten der Gläubigergesamtheit stellt dabei den Unterschied zwischen Einzelvollstreckung und der Gesamtvollstreckung dar, ${ }^{91}$ wodurch die Annahme eines Insolvenzplans gegen den Widerspruch einzelner Gläubiger vollzogen werden kann (vgl. § 244 InsO).

Jedenfalls sind die einzelnen Aufgaben des Insolvenzgerichts ihrem Wesen und ihrer Struktur nach nicht nur typisch prozessuale, ${ }^{92}$ sondern enthalten auch administrative und rechtsfürsorgliche Züge, die dem Prozessverfahren der freiwilligen Gerichtsbarkeit zugeordnet werden können. Dabei obliegt dem Insolvenzgericht, z.B. neben der Sorge für die äußere Ordnung des Verfahrensablaufes (etwa Bestimmung von Terminen und Fristen §§ 29, 235 InsO) und der Einberufung sowie der Leitung der Gläubigerversammlungen (§§ 29, 74f., 76, 235, $241 \mathrm{InsO}$ ), als wichtigste Aufgabe die Überwachung der Gesetzmäßigkeit und Ordnungsmäßigkeit des Verfahrensablaufes und die Aufsicht über den Insolvenzverwalter (§ $58 \mathrm{InsO}) .{ }^{93}$ Neben diesen Aufgaben des Insolvenzgerichts sind auch der im Insolvenzverfahren geltende Untersuchungsgrundsatz (§ 5 I InsO) und die freigestellte mündliche Verhandlung vor Entscheidungen (§ 5 III InsO) typische Merkmale der freiwilligen Gerichtsbarkeit.

Hess, InsO, Vor § 2 Rn.19.

Siehe bereits unter C.I. (S.10ff.).

Z.B. im Rahmen der Verfahrenseröffnung/-beendigung (§§ 27, 200, 212f., 258 InsO) und der Anordnung von Sicherheits- und Zwangsmaßnahmen ( $\S$ 21, 28, 98 InsO). 
Die voranstehenden Ausführungen zeigen zusammenfassend, dass das Insolvenzverfahren als Gesamtvollstreckungsverfahren ganz unterschiedliche Elemente in sich vereint und damit eine Sonderstellung innerhalb prozessualer Verfahrensordnungen einnimmt. Den Einstieg in das Insolvenzverfahren bildet aber gleichwohl die zwangsweise Verwertung und Verteilung des schuldnerischen Vermögens, was den Charakter einer Zwangsvollstreckung durch die Berücksichtigung sozialer Aspekte nicht ändert.

Daher bleibt festzuhalten, dass das Insolvenzverfahren und auch das Anmelde- und Feststellungsverfahren der $\S \S$ 174ff. InsO als selbstständiges Gesamtvollstreckungsverfahren grundlegend der streitigen Gerichtsbarkeit zuzuordnen ist, ${ }^{94}$ wobei es aber kein Erkenntnisverfahren ist. Insoweit bedarf das Insolvenzverfahren trotz seiner weit größeren Nähe zur streitigen Gerichtsbarkeit ${ }^{95}$ bei der Auslegung der allgemeinen Vorschriften und der entsprechenden Heranziehung der Zivilprozessordnung ( $\S 4$ InsO) im Einzelfall einer Überprüfung der Zusammenhänge nach Sinnverwandtschaft und Vereinbarkeit mit dem Zweck bzw. den Verfahrensgrundsätzen des Insolvenzverfahrens. ${ }^{96}$

Siehe auch Darstellung bei Merkle, RPfleger 2001, 157 (159ff.) m.w.N.

Kritisch zur Problematik einer systematischen Einordnung siehe Baur/Stürner, Insolvenzrecht, Rn. 1.11f.; Kilger/K.Schmidt, KO, § 71 Anm.1; Schmidt, KTS 1988, 1 (2ff.).

96 So auch MüKo-InsO/Ganter, Vor §§ 2-10 Rn.9; Jaeger/Gerhardt, § 2 Rn.18; GrafSchlicker/Kexel, § 4 Rn.2. 


\section{Anmeldeerfordernis}

Im Folgenden sollen zunächst das allgemeine Anmeldeerfordernis und die damit verbundene (III.) Aufforderung zur Anmeldung als Einstieg in das Anmeldeverfahren erörtert werden, um anschließend (IV.) auf die einzelnen Anforderungen an eine ordnungsgemäße und wirksame Anmeldung eingehen zu können.

$\S 174$ I 1 InsO normiert ein Anmeldeerfordernis von allen, auch bereits titulierten (vgl. § 179 II InsO) Insolvenzforderungen zur Insolvenztabelle durch den Insolvenzgläubiger, so dass das Insolvenzgläubigerrecht auf Befriedigung aus der Insolvenzmasse nicht von Amts wegen berücksichtigt wird. ${ }^{97}$ Die Anmeldung rückt - wie auch das Zivilgericht nur auf Antrag urteilt (§ 308 I 1 ZPO) - in die Nähe der Klageerhebung (§ 253 I ZPO) oder der sonstigen je nach Rechtsgebiet vorgesehenen gerichtlichen oder behördlichen Schritte zur Anspruchsverwirklichung; ${ }^{98}$ auch wenn dadurch eine Rechtshängigkeit nicht begründet wird. ${ }^{99}$ Hierin zeigt sich die zuvor bereits dargestellte ${ }^{100}$ Sonderstellung des Insolvenzverfahrens innerhalb der prozessualen Verfahrensordnungen. Das Anmeldeerfordernis wird auch nicht durch bereits vorliegende Kenntnisse von einer Gläubigerstellung seitens der Verfahrensbeteiligten oder des Insolvenzgerichts aufgrund des gem. § 152 I InsO vom Insolvenzverwalter in Zusammenarbeit mit dem Schuldner separat zu erstellende Gläubigerverzeichnis ersetzt. ${ }^{101}$ Eine Ausnahme zum Anmeldeerfordernis besteht also nicht.

Es handelt sich hier aber nicht um eine „echte“ Pflicht zur Insolvenzforderungsanmeldung eines jeden Gläubigers, sondern einer der

97 Uhlenbruck/Uhlenbruck, § 174 Rn.1; KS/Eckardt, S.749 Rn.11; Gerke, ZinsO 2003, 873 (873f.).

98 Vgl. bereits unter B. (S.4ff.); MüKo-InsO/Nowak, § 174 Rn.2; Pape/Uhlenbruck, InsO, Rn.104; Becker, Rn.1245, 210ff.; Mäusezahl, ZInsO 2002, 462 (463) m.w.N.

99 Dies erfolgt erst bei einer evtl. Klageerhebung i.R.d. §§ 179ff. InsO. Siehe auch unter D.VI. (S.94ff.).

100 Siehe unter D.I. (S.23ff.).

101 Kübler/Prütting/Bork/Pape, § 174 Rn.8; Nerlich/Römermann/Becker, § 174 Rn.8; Pape/Uhlenbruck, Rn.720. Umgekehrt, soll die Anmeldung jedoch ihrerseits das Gläubigerverzeichnis ergänzen, § 152 I Var.3 InsO. Denn das Gläubigerverzeichnisse (§ 152 InsO) ist zusammen mit dem aufzustellenden Massenverzeichnis ( 151 InsO) Basis der nach $\S 153$ InsO zu fertigenden Vermögensübersicht, die den Gläubigern die Beurteilung der Vermögenslage des Schuldners ermöglichen soll. Insolvenztabelle und Gläubigerverzeichnis sind daher grundsätzlich voneinander zu trennen. - Siehe auch Begr. zu $\S 170$ (§ 151 InsO) RegE., BT-Drucks. 12/2443, S.171. 
Entscheidungsfreiheit ${ }^{102}$ des Einzelnen zugrundeliegende, im eigenen Interesse wahrzunehmende verfahrensrechtliche Obliegenheit (Last). ${ }^{103}$ Unterbleibt jedoch die Forderungsanmeldung, so ist der Insolvenzgläubiger zwar von einer Mitwirkung und Verfolgung seiner Interessen im Insolvenzverfahren ausgeschlossen, ${ }^{104}$ unterliegt aber gleichwohl - aufgrund seiner materiellen Insolvenzgläubigerstellung - dessen allgemeinen verfahrensrechtlichen Wirkungen (§§ 87, 89 InsO). ${ }^{105}$ Dennoch ist eine Nichtteilnahme am Verfahren wenig sinnvoll. Im Gegenteil drohen dem Insolvenzgläubiger Nachteile nicht nur bei Annahme und Bestätigung eines Insolvenzplans (§ 254 I 1, 3 InsO), sondern vor allem für das Restschuldbefreiungsverfahren, denn gem. § 301 I InsO tritt die Restschuldbefreiung gegenüber sämtlichen Insolvenzgläubigern ein, und zwar unabhängig von der Frage, ob sie ihre Forderungen angemeldet haben oder nicht (§ 301 I 2 InsO). Jedoch sind Insolvenzforderungen aus vorsätzlich begangener unerlaubter Handlung des Schuldners gem. § 302 Nr.1 InsO von der Restschuldbefreiung ausgeschlossen. Dies aber wiederum nur, soweit sie auch angemeldet worden sind (§ 302 Nr.1 2.Halbsatz InsO) und der Schuldner ihnen nicht erfolgreich widersprochen hat und ein Widerspruch im Feststellungsverfahren auch nicht beseitigt werden konnte ( $\S \S 179 \mathrm{ff}$. InsO). ${ }^{106}$ Auf die in diesem Zusammenhang bestehende Hinweispflicht des Insolvenzgerichts gegenüber dem Schuldner gem. § 175 II InsO soll erst an entsprechender Stelle des Verfahrensablaufes näher eingegangen werden. ${ }^{107}$ Zunächst bleibt aber festzuhalten, dass derjenige Insolvenzgläubiger, der die Anmeldung seiner Insolvenzforderung

102 Diese Dispositionsmaxime ist allgemeiner Verfahrensgrundsatz und der Zivilprozessordnung immanent. Siehe etwa bei Zöller/Greger, ZPO, Vor § 128 Rn.2, 9.

103 Nerlich/Römermann/Becker, § 174 Rn.7; MüKo-InsO/Nowak, § 174 Rn.1; Uhlenbruck/Uhlenbruck, § 174 Rn.1; Häsemeyer, InsO, Rn.22.08; KS/Eckardt, S.749 Rn.11; Mohrbutter/Ringstmeier/Ernestus, § 11 Rn.1; Pape/Uhlenbruck, Rn.121; Becker, Rn.194, 1245.

104 BGHZ 72, 234 (234); BGH NJW 1972, 162 (162); Jaeger/Henckel, KO, § 12 Rn.3, 8; Uhlenbruck/Uhlenbruck, § 174 Rn.11 m.w.N.

105 Siehe bereits unter C.I. (S.10ff.); Kübler/Prütting/Bork/Pape, § 174 Rn.8; Uhlenbruck/Uhlenbruck, § 174 Rn.11; Breuer, Rn.396; allgemein zu den Problemen der Nichtteilnahme am und im Insolvenzverfahren siehe ausführlich bei Birkenau, S.78ff.

106 Siehe dazu unter E.III.5.c) (S.225ff.).

107 Zur Hinweispflicht des Insolvenzgerichts gem. § 175 II InsO siehe unter D.V.5. (S.90ff.). 
unterlässt, auch nicht die Vorteile des Insolvenzverfahrens genießt, aber allen verfahrensrechtlichen Wirkungen der Insolvenzordnung unterfällt. 


\section{Aufforderung zur Anmeldung}

Auf das Anmeldeerfordernis werden die Insolvenzgläubiger im Eröffnungsbeschluss hingewiesen, indem sie gem. § 28 I 1 InsO vom Insolvenzgericht aufgefordert werden, ihre Insolvenzforderungen innerhalb einer bestimmten Frist $^{108}$ unter Beachtung des $\S 174$ InsO beim Insolvenzverwalter anzumelden. Der Eröffnungsbeschluss ist durch die Geschäftsstelle des Insolvenzgerichts sofort öffentlich bekannt zu machen (§ 30 I 1 InsO). Dies geschieht neuerdings ausschließlich zentral und länderübergreifend im Internet ${ }^{109}$ (§ 9 I 1 InsO). ${ }^{110}$ Eine Veröffentlichung im Bundesanzeiger findet nicht mehr statt. ${ }^{111}$ Zusätzlich ist der Beschluss gem. § 30 II InsO, soweit und sobald ihre Anschrift oder die ihrer Vertreter (§ 8 II 2 InsO) dem Insolvenzgericht bekannt ist, dem Schuldner und dessen Gläubigern und Schuldnern vollständig ${ }^{112}$ und gesondert zuzustellen. ${ }^{113}$ Die Zustellung kann und wird in der Praxis regelmäßig gem. § 8 III InsO dem Insolvenzverwalter übertragen werden.

Im Fall eines Antrages auf Restschuldbefreiung des Schuldners bei Verfahrenseröffnung ( $§ 287$ I 1 InsO) ist hierauf, wenn dies nicht bereits mit dem Eröffnungsbeschluss geschehen ist (§ 27 II Nr.4 InsO), bei der Bekanntmachung gem. § 30 I 2 InsO gesondert hinzuweisen. Jedoch lässt $\S 9$ III InsO, auch wenn die Insolvenzordnung wie in $\S 30$ II InsO die besondere Zustellung vorschreibt, die öffentliche Bekanntmachung im Internet für den Nachweis der Zustellung an alle Beteiligten ausreichen. ${ }^{114}$ Insofern ist auch eine fehlende individuelle Unterrichtung über das Verfahren schadlos. ${ }^{115}$

108 Dazu unter D.IV.7. (S.72ff.).

109 Unter www.insolvenzbekanntmachungen.de

110 Siehe hierzu: Verordnung zu öffentlichen Bekanntmachung im Insolvenzverfahren im Internet (InsoBekV), vom 12.2.2002 (BGBl. I S.677). Zudem ist die Übergangsvorschrift des Art. 103c EGInsO zu beachten.

111 Dadurch sollen Bekanntmachungskosten eingespart werden. Begr. zu Nr.3a (§ 9 InsO) RegE, BT-Drucks. 16/3227, S.13f.

112 Etwas anderes lässt der Gesetzeswortlaut nicht zu. FK/Schmerbach, § 30 Rn.14; Kübler/Prütting/Bork/Pape, § 30 Rn.5; Graf-Schlicker/Graf-Schlicker/Kexel, § 30 Rn.9; HK/Kirchhof, § 30 Rn.8 m.w.N.

113 Dass selbstverständlich auch der Insolvenzverwalter über den Inhalt des Eröffnungsbeschlusses zu unterrichten ist (so noch ausdrücklich § 6 I 2 GesO), braucht nach Ansicht des Gesetzgebers im Gesetz nicht mehr gesondert erwähnt werden. - Begr. zu § 36 (§ 30 InsO) RegE, BT-Drucks. 12/2443, S.120.

114 Bedeutsam für den Beginn der Beschwerdefrist des $\S 6$ II InsO.

115 KS/Eckardt, S. 749 Rn.11; Gottwald/Eickmann, § 63 Rn.43; Jaeger/Schliken, InsO, $\S 28 \mathrm{Rn} .5$. 
Anders hingegen verhält es sich bei ausländischen, der europäischen Insolvenzordnung ${ }^{116}$ unterliegenden Gläubigern der EUMitgliedsstaaten. ${ }^{117}$ Gleichgültig, ob das deutsche Insolvenzverfahren Hauptinsolvenzverfahren (Art. 3 I EuInsVO) ist oder Territorialverfahren (Art. 3 II EuInsVO; § 354 InsO), können ausländische Gläubiger mit ihren Insolvenzforderungen genauso wie inländische am Verfahren teilnehmen (Art. 32 I, 36, 39 EuInsVO; § 341 I InsO). Für das Anmeldeverfahren ${ }^{118}$ bestimmen die Art. 40, 42 EuInsVO; Art. $102 \S 11$ EGInsO jedoch, dass für bekannte Insolvenzgläubiger, die ihren gewöhnlichen Aufenthalt, Wohnsitz oder Sitz in einem anderen Vertragsstaat haben, das Insolvenzgericht oder der zuständige Insolvenzverwalter besondere Unterrichtungen an die auswärtigen Insolvenzgläubiger zu versenden hat, um ihnen das Anmelden ihrer Forderungen nahe zu legen. Die Unterrichtung hat anhand eines Formblattes gem. Art. 40 I 2, II EuInsVO in diesem Fall anzugeben, welches die Versäumnisfolgen sind, welche Stelle für die Entgegennahme der Anmeldung zuständig ist und welche weiteren Maßnahmen vorgeschrieben sind. Auf Einzelheiten wird noch vertiefend an anderer Stelle einzugehen sein ${ }^{119}$

116 EG-Verordnung Nr.1346/2000 vom 29.05.2000, in Kraft getreten am 31.05.2002; EG-ABl. L 160 vom 30.06.2000, S. 1 ff.

117 Siehe hierzu allgemein: Wimmer, ZInsO 2001, 97ff.; Kemper, ZIP 2001, 1609ff.

118 Ergibt sich nach Art.4 II 2 h EuInsVO; § 335 InsO aus dem deutschen Insolvenzrecht.

119 Siehe unter D.IV.2.a) (S.36ff.) und D.IV.3.e) (S.48ff.). 


\section{Anforderungen an die Anmeldung}

Im Folgenden sollen die einzelnen Anforderungen an die Anmeldung behandelt werden. Dabei soll insbesondere auf die Problematik des Unterschriftenerfordernisses und die Einreichung von Originalurkunden eingegangen werden.

\section{Adressat der Anmeldung}

In Anlehnung an $\S \S 5$ S.2 Nr.3, 11 I, 14 GesO, aber abweichend vom vormaligen $\S 139$ S.2 KO, bestimmen die $\S \S 28$ I 1, 174 I 1 InsO, dass die Anmeldung der Insolvenzforderungen beim Insolvenzverwalter zu erfolgen hat. Dies soll der Entlastung der Insolvenzgerichte dienen ${ }^{120}$ und dem Insolvenzverwalter die wichtige Aufgabe erleichtern, ein Gläubigerverzeichnis gem. § $152 \mathrm{InsO}^{121}$ aufzustellen. Eine Anmeldung beim Insolvenzgericht ist daher nicht zulässig und als solche nicht wirksam. Sollte eine Anmeldung dennoch beim Insolvenzgericht eingereicht werden, ${ }^{122}$ so ist dieses von Amts wegen ( $\$ 5 \mathrm{I}$ InsO) und aus Gründen eines fairen Verfahrens ${ }^{123}$ gehalten, die Anmeldung direkt an den Insolvenzverwalter weiterzuleiten und den Anmeldenden darüber zu informieren. ${ }^{124}$

Im Falle der Eigenverwaltung (§ 270 I 1 InsO) wird die Anmeldung beim Sachwalter angebracht (§ 270 III 2 InsO). Im vereinfachten Verbraucherinsolvenzverfahren ist der Adressat der Treuhänder (§§ 313 I 1, 292 InsO).

120 So der Rechtsauschuss zu $\S 32$ (§ 28 InsO), BT-Drucks. 12/7302, S.159, wonach $\S 5$ S.2 Nr.3 GesO als Vorbild dient.

121 Siehe auch Fn.101 (S.30).

122 Dies gilt auch für verfrühte Anmeldungen. Siehe dazu unter D.IV.7.a) (S.73f.).

23 BVerfGE 46, 325 (334f.); 52, 131 (145f.); 67, 329 (339).

124 Uhlenbruck/Uhlenbruck, § 174 Rn.12; MüKo-InsO/Nowak, $\S 174$ Rn.27; Kübler/Prütting/Bork/Pape, § 174 Rn.25; Jaeger/Schliken, InsO, § 28 Rn.10. 


\section{Die wesentliche Form der Forderungsanmeldung}

\section{a) Schriftform und Sprache}

Die Anmeldung der Insolvenzforderung hat gem. § 28 I 1 i.V.m. § 174 I 1 InsO schriftlich beim Insolvenzverwalter zu erfolgen. Die Möglichkeit einer mündlichen Anmeldung, wie sie nach $\S 139$ KO beim Urkundsbeamten der Geschäftsstelle des Gerichts möglich war, entfällt damit.

Auch wenn die Forderungsanmeldung beim Insolvenzverwalter erfolgt, ist sie die verfahrensrechtliche Geltendmachung des materiellen Anspruchs des Insolvenzgläubigers gegen den Schuldner im Prüfungs- und Feststellungsverfahren und entspricht der Bedeutung einer Klageschrift bzw. eines Mahnantrages. ${ }^{125}$ Sie stellt damit eine Prozesshandlung dar, worunter man jedes äußere Verhalten einer Partei versteht, das nach seinem Sinn darauf abzielt, eine für die Handlung charakteristische verfahrensrechtliche Wirkung - in diesem Fall die Teilnahme am Insolvenzverfahren - herbeizuführen. ${ }^{126}$ Als Prozesshandlung ist die Insolvenzanmeldung damit grundsätzlich in deutscher Schrift abzufassen (§ 184 GVG analog); ${ }^{127}$ sie unterliegt den Vorschriften der Zivilprozessordnung ( $§ 4 \mathrm{InsO}) .{ }^{128}$

Gläubiger aus anderen EU-Mitgliedsstaaten können gem. Art. 42 II EuInsVO die Forderungen jedoch auch in ihrer Amtssprache oder einer der Amtssprachen der anderen Staaten anmelden. In diesem Fall muss die Anmeldung aber mindestens die Überschrift „Anmeldung einer Forderung“ in deutscher Sprache enthalten. Zudem kann vom Gläubiger

125 Die Anmeldung enthält jedoch keine Zahlungsaufforderung und begründet auch keinen Verzug; dies weder gegenüber dem Schuldner, der ohnehin nicht über sein Vermögen verfügen darf (§ 80 I InsO), noch gegenüber dem Insolvenzverwalter, da eine Anmeldung zur Hemmung des Anspruchs führt (§ 204 I Nr.10 BGB). Siehe auch D.VI.4. (S.115ff.). - RGZ 121, 207 (211); MüKo-InsO/Nowak, § 174 Rn.2; GrafSchlicker/Graf-Schlicker, § 174 Rn.2; Kübler/Prütting/Bork/Pape, § 174 Rn.37; vgl. auch FK/Kießner, § 174 Rn.11; KS/Eckardt, S.750 Rn.13.

126 Stein/Jonas/Leipold, ZPO, Vor § 128 Rn.211; Zöller/Greger, ZPO, Vor § 128 Rn.14.

127 Die Maßgeblichkeit des Gerichtsverfassungsgesetzes (GVG) für das Insolvenzverfahren setzt das Recht als selbstverständlich voraus. Siehe dazu bereits in Materialien und Motive zum GVG bei Hahn, GVG, S.189, 370; vgl. auch Art.12 EGInsO; weiter RGZ 31, 428 (428); FK/Kießner, § 174 Rn.13; Jaeger/Gerhardt, InsO, § 4 Rn.2; Kübler/Prütting/Bork/Pape, § 174 Rn.25; Uhlenbruck/Uhlenbruck, $\S 4$ Rn.40, § 174 Rn.14 m.w.N.

128 Zöller/Greger, ZPO, Vor § 128 Rn.15; Stein/Jonas/Leipold, ZPO, Vor § 128 Rn.231ff. 
verlangt werden, dass eine Übersetzung der Anmeldung ins Deutsche nachgereicht wird (Art. 42 II 3 EuInsVO). Von dieser Befugnis hat der Insolvenzverwalter grundsätzlich Gebrauch $\mathrm{zu}$ machen und eine Übersetzung nachzufordern, da sonst eine eingehende Prüfung - insbesondere auch für die übrigen Verfahrensteilnehmer - nicht möglich sein wird.

Vor dem Hintergrund des Art. 42 II EuInsVO und einer Welt der fortschreitenden Globalisierung und wirtschaftlichen Vernetzung erscheint es trotz fehlender gesetzlicher Regelung nicht gerechtfertigt, Insolvenzanmeldungen, die in einer fremden Sprache verfasst sind bzw. von Insolvenzgläubigern mit gewöhnlichem Sitz außerhalb des Anwendungsbereichs des EU-Übereinkommens angemeldet werden, generell als unzulässig anzusehen bzw. im Rahmen des Anmeldeverfahrens gänzlich unberücksichtig zu lassen. ${ }^{129}$ Dies erscheint zumindest dann nicht gerechtfertigt, wenn man die oben genannten Mindestanforderungen des $\S 42$ II 2 EuInsVO (Amtssprache eines der Mitgliedsstaaten und deutsche Überschrift „Anmeldung einer Forderung“) heranzieht und der angebrachte Schriftsatz dadurch eindeutig erkennbar als Insolvenzforderungsanmeldung qualifiziert werden kann, ${ }^{130}$ da auch hinsichtlich des ausländischen Insolvenzgläubigers - der kein EU-Bürger ist - der spezifische Grundsatz der gemeinschaftlichen Gläubigerbefriedigung und -gleichbehandlung für das Insolvenzverfahren einen besonderen Stellenwert einnimmt. Um aber die Prüfung der angemeldeten Insolvenzforderung zu ermöglichen und damit letztlich auch § 184 GVG gerecht zu werden, hat der Insolvenzverwalter - wie es auch Art. 42 II 3 EuInsVO vorsieht - den Anmeldenden daher stets aufzufordern binnen angemessener Frist eine Übersetzung der Anmeldung nachzureichen. ${ }^{131}$ Sollte dagegen selbst dieses Mindestmaß an Erkennbarkeit einer Insolvenzforderungsanmeldung nicht gegeben sein, etwa weil der

129 So aber Kübler/Prütting/Bork/Pape, § 174 Rn.25; HK/Irschlinger, § 174 Rn.2; BK/Breutigam § 174 Rn.11; Hess/Weis/Wienberg/Weis, InsO, § 174 Rn.37.

130 Ähnlich und teilweise weitergehend Gottwald/Eickmann, § 63 Rn.12; KS/Eckardt, S.750 Rn.13; Bähr, InVo 1998, 205 (207); Smid/Smid, § 174 Rn.12; wohl auch Uhlenbruck/Uhlenbruck, § 174 Rn.14.

131 Dies sollte in jedem Fall noch vor dem Prüfungstermin geschehen, damit allen Verfahrensteilnehmern die Prüfung der angemeldeten Insolvenzforderung bis zum Beginn des Prüfungstermins möglich ist. 
Schriftsatz komplett auf Chinesisch verfasst ist, kann sie nicht als zum Verfahren vorgenommen angesehen werden.

\section{b) Unterschrifterfordernis}

Die Forderungsanmeldung ist gem. § 174 I 2 InsO schriftlich beim Insolvenzverwalter anzubringen. Auch wenn sie gegenüber dem Insolvenzverwalter erfolgt, stellt sie grundsätzlich eine Verfahrenshandlung dar, sodass über $\S 4$ InsO die Grundsätze für Schriftsätze der $\S \S 129 f f$. ZPO entsprechend zur Anwendung kommen. Im Folgenden soll auf die wenig behandelte Frage eingegangen werden, ob das Unterschriftenerfordernis gem. $\quad \S \quad 130 \quad$ Nr.6 $\quad$ ZPO auch für die Insolvenzforderungsanmeldung einzuhalten ist.

Zunächst ist festzuhalten, dass die Anmeldung beim Insolvenzverwalter nicht lediglich ein vorbereitender Schriftsatz ist, der zur Ankündigung der Anmeldung oder eines anderen Vortrages dient, sondern eine eindeutige und damit bestimmende Erklärung des Insolvenzgläubigers enthält, mit dessen Einreichung beim Insolvenzverwalter eine wesentliche, den Gang des Verfahrens bestimmende Prozesshandlung vorgenommen wird, nämlich die konkrete Anmeldung zur Teilnahme am Verfahren der $\S \S 174 \mathrm{ff}$. InsO. ${ }^{132}$ Bei der Insolvenzforderungsanmeldung handelt es sich also um einen bestimmenden Schriftsatz, weshalb die Grundsätze für bestimmende Schriftsätze im Rahmen der §§ 129ff. ZPO i.V.m. § 4 InsO maßgeblich sind.

Bei der Regelung des Unterschrifterfordernisses gem. § 130 Nr.6 ZPO handelt es sich zwar um eine Sollvorschrift, die jedoch nach herrschender Meinung bei bestimmenden Schriftsätzen, im Gegensatz zu den nur vorbereitenden Schriftsätzen, generell als Wirksamkeitsvoraussetzung angesehen wird. ${ }^{133}$ Dadurch soll feststehen, dass es sich um eine verfahrensrechtlich gewollte Erklärung handelt, die vom Unterzeichner herrührt und für die er die Verantwortung übernimmt, da insoweit - anders

2 Solche Erklärungen, die ein Verfahren oder Verfahrensteilnahme eröffnen, sind grundsätzlich bestimmende Schriftsätze. Siehe nur Thomas/Putzo/Reichold, § 129 Rn.1, 5; Musielak/Stadler, ZPO, § 129 Rn.6 m.w.N.

133 Grundlegend RGZ 31, 375 (377); 151, 82 (83); weiter BGHZ 37, 156 (157); 97, 283 (284f.); für die Literatur siehe nur Stein/Jonas/Leipold, ZPO, § 130 Rn.14 m.w.N. 
als bei nur vorbereitenden Schriftsätzen - die gebotene Rechtssicherheit keinen Streit über Urheberschaft und prozessuale Verbindlichkeit duldet und eine sichere Abgrenzung zum versehentlichen aus der Hand gegebenen Entwurf möglich sein muss. ${ }^{134}$ Gleichwohl werden zunehmend Stimmen lauter, ${ }^{135}$ die das scheinbar einfache Unterschrifterfordernis aufgrund der Vielzahl kaum noch überschaubarer Einzelausnahmen als Wirksamkeitserfordernis generell aufgeben und zur einfachen Sollvorschrift zurückkehren wollen. So wird auch hinsichtlich der Anmeldung zur Insolvenztabelle trotz fehlender Unterschrift die zweifelsfreie Erkennbarkeit des Absenders für eine ordnungsgemäße Anmeldung als ausreichend erachtet. ${ }^{136}$ Dem kann jedoch hinsichtlich der eindeutigen Stellungnahme des Gesetzgebers hinsichtlich dieser Streitfrage in jüngerer Zeit nicht gefolgt werden.

Insbesondere die Einführung des $\S 174$ IV InsO durch Art. 9 des Justizkommunikationsgesetzes (JKomG) ${ }^{137}$ im Jahr 2005 und dessen Bezug zu § 130a ZPO zeigt deutlich, dass der Gesetzgeber gerade nicht von dieser grundsätzlichen Wirksamkeitsvoraussetzung der Unterschrift - sei es in schriftlicher oder elektronischer Form - Abstand nehmen wollte. Gemäß $\S 174$ IV 1 InsO kann die Anmeldung alternativ zur herkömmlichen Schriftform nämlich nun auch durch Übermittlung eines elektronisches Dokumentes (z.B. E-Mail oder Datenträger) erfolgen. ${ }^{138}$ Ausschlaggebend für die Problematik des Unterschriftenerfordernisses ist hierbei jedoch, dass auch im Fall der Übermittlung durch elektronische Dokumente § 130a I 2 ZPO selbst für nur vorbereitende Schriftsätze ausdrücklich fordert, dass die elektronischen Dokumente von der verantwortlichen Person mit einer

134 MüKo-InsO/Nowak, § 174 Rn.9;

135 Musielak/Stadler, ZPO, § 129 Rn.8f.; Zöllner/Greger, § 130 Rn.21f., ausführlich dazu Heinemann, S.338. Jeweils mit Blick auf das Urteil zur Zulässigkeit von Computerfax-Schriftsätzen aus dem Jahr 2000, GmS-OGB BGHZ 144, $160 \mathrm{ff}$. Grundlegend auch Vollkommer, Formstrenge, S.260ff.

136 Graf-Schlicker/Graf-Schlicker, § 174 Rn.11; Uhlenbruck/Uhlenbruck, § 174 Rn.13; Kübler/Prütting/Bork/Pape, § 174 Rn.25; Mohrbutter/Ringstmeier/Ernestus, § 174 Rn.3.

137 Gesetz über die Verwendung elektronischer Kommunikationsformen in der Justiz (Justizkommunikationsgesetz - JKomG) vom 22. März 2005, BGBl. I S.837 (851f).

138 Dies aber auch nur dann, wenn der Insolvenzverwalter der elektronischen Übermittlung ausdrücklich zugestimmt hat, weil er die Sicherung und Archivierung der übersendeten Daten sicherstellen kann. Für Einzelheiten hinsichtlich bestimmter Formerfordernisse für die Weiterverarbeitung der elektronischen Dokumente (z.B. Dateiformat) bei Gericht sind die von Bundes- und Landesregierungen zu erlassenden Verordnungen zu beachten, § 130a II ZPO. 
qualifizierten elektronischen Signatur nach dem Signaturgesetz ${ }^{139}$ versehen werden sollen. Auch wenn laut Gesetzesbegründung die bestimmenden Schriftsätze - wie die Forderungsanmeldung - ausdrücklich von dieser Regelung mit umfasst sein sollen, ${ }^{140}$ es sich wie bei der Schriftformregelung des $\S 130$ Nr.6 ZPO aber ebenfalls „nur“ um eine Sollvorschrift handelt, stellt die in § 130a I 2 ZPO geforderte qualifizierte elektronische Signatur nach den Vorstellungen des Gesetzgebers ebenso wie die Unterschrift bei Schriftform nach § 130 Nr.6 ZPO weiterhin ein zwingendes Wirksamkeitserfordernis dar. ${ }^{141}$ Eine Korrektur der bekannten und umfangreichen Rechtsprechung zum generellen Unterschriftserfordernis ist laut der Regierungsbegründung zum Gesetzesentwurf des JKomG ausdrücklich nicht beabsichtigt gewesen. ${ }^{142}$ Insofern ist insgesamt an dem grundsätzlichen Unterschrifterfordernis festzuhalten. ${ }^{143}$

Dies hat - aufgrund der Einordnung als bestimmender Schriftsatz, ${ }^{144}$ dem entsprechenden Bezug zu den $\S \S 129 \mathrm{ff}$. ZPO über $\S 4$ InsO, als auch über § 174 IV InsO speziell zu § 130a ZPO - folglich auch entsprechend für die Insolvenzforderungsanmeldung zu gelten. Zwar sieht § 4 InsO lediglich die entsprechende Anwendung der Vorschriften der Zivilprozessordnung vor, ${ }^{145}$ so dass man argumentieren könnte, dass aufgrund der speziellen Zuweisung durch § 174 IV InsO allein § 130a ZPO hinsichtlich der dort geregelten und vielmehr aus sicherheitstechnischen Gründen einzuhaltenden Übertragungsweise von elektronischen Dokumenten im Insolvenzverfahren Anwendung finden soll, und die sonstigen §§ 129ff. ZPO - insbesondere § 130 Nr.6 ZPO - aufgrund eines fehlenden

139 § 2 Nr.3 i.V.m. Nr.2 SigG, Signaturgesetz vom 16. Mai 2001 (BGBl. I S. 876).

140 Begr. zu § 130a RegE, BT-Drucks. 14/4987, S.24.

141 Im Anschluss an die Diskussion im Vermittlungsausschuss, siehe Plenarprotokoll BR (765.Sitzung), 22. Juni 2001, S.322; siehe auch Stein/Jonas/Leipold, ZPO, $\S$ 130a Rn.11; Musielak/Stadler, ZPO, § 130a Rn.3; Hartmann, NJW 2001, 2577 (2778); Dästner, NJW 2001, 3469 (3470).

142 So ausdrücklich Begr. zu § 130a RegE, BT-Drucks. 14/4987, S.23f. speziell mit Bezug zu GmS-OGB BGHZ 144, 160ff. In einem späteren Urteil (BGH NJW 2005, 2086) findet bereits ein Relativierung des GmS-OBG statt, so dass BGHZ 144, $160 \mathrm{ff}$. wohl als Ausnahme angesehen werden kann.

143 Der von Vollkommer, Formstrenge, S.126ff., S.260ff., vertretenen und in diesen Zusammenhängen immer wieder aufgeworfene Ansicht, dass Unterschriftenmängel bei evidenter Erreichung des Formzwecks unschädlich seien, tritt somit nicht mehr nur die (ständige) Rechtsprechung und h.M. in der Literatur, sondern offensichtlich auch der Gesetzgeber entgegen. Indem der allgemeinen Rechtssicherheit der Vorzug (vor dem Streben nach Einzelfallgerechtigkeit) gegeben wird.

144 Dies zeigt die Nähe zur Klageschrift/-erhebung (§ $253 \mathrm{ZPO}$ ), siehe bereits unter D.II. (S.30ff.).

145 Dazu bereits unter D.I.3. (S.27ff.). 
Verweises gerade nicht. Jedoch verhält es sich vielmehr umgekehrt, dass gerade die grundsätzliche Anwendung der §§ 129ff. ZPO i.V.m. § 4 InsO im Insolvenzverfahren eine Ergänzung hinsichtlich § 130a ZPO erforderlich macht. Der Insolvenzverwalter übt lediglich ein vom Insolvenzgericht begründetes Amt mit den sich aus der Insolvenzordnung und dem Verfahren ergebenden Aufgaben aus, ${ }^{146}$ denen er in seinem eigenen Aufgaben- und Wirkungskreis nachkommt. Die notwendige elektronische Umsetzung und Einrichtung, die $\S 130$ a ZPO durch Rechtsverordnungen des Bundes und der Länder eröffnet, betrifft jedoch die Gerichte und deren ortsgebundene (mediale) Organisation und umfasst gerade nicht (auch) die Organisationsstrukturen des Insolvenzverwalters. Anders als beim untechnischen und damit ortsungebunden durchführbaren Schriftverkehr, dessen prozessualen Voraussetzungen (§§ 174 I, 4 InsO i.V.m. $§ \quad 130$ Nr.6 ZPO) ohne weiteres auch gegenüber dem Insolvenzverwalter eingehalten werden können, ist dies im Hinblick auf § 130a ZPO im elektronischen Verfahren gerade nicht gegeben bzw. kann nicht ohne weiteres bei allen Insolvenzverwaltern vorausgesetzt werden. Um gleichwohl die elektronische Anmeldung ohne Analogie zu § 130a ZPO beim Insolvenzverwalter zu ermöglichen, wurde $\S 174$ IV InsO als Ergänzung eingeführt. Die Einschränkung, dass der Insolvenzverwalter der elektronischen Übermittlung der Anmeldung ausdrücklich zugestimmt haben muss, stellt dabei sicher, dass elektronische Anmeldungen nur in einer vom Insolvenzverwalter in seinem Organisationskreis verwendbaren Form erfolgen. ${ }^{147}$ Insofern ist $\S 174$ IV InsO nicht als Ausnahme zur Anwendbarkeit der $\S \S 129 f f$. ZPO über $\S 4$ InsO, sondern als deren (notwendige) Ergänzung hinsichtlich der Einbeziehung auch des § 130a ZPO bei der Anmeldung zu sehen.

Daher bleibt festzuhalten, dass für eine wirksame Forderungsanmeldung im Insolvenzverfahren grundsätzlich eine Unterschrift zu fordern ist, sei es in Schriftform (§ 130 Nr.6 ZPO) oder als qualifizierte elektronische Signatur $^{148}$ (§ 130a I 2 ZPO). Sie soll nämlich nicht nur aus allgemeinen

Siehe dazu unter D.IV.5.d) (S.65ff.) und E.I.2.c) (S.136ff.).

147 Siehe auch Begr. zu Art.9 Nr.2 (§ 174 IV InsO) RegE, BT-Drucks. 15/4067, S.54.

148 Diese ist bei einem Zertifizierungsanbieter $\mathrm{zu}$ beantragen (z.B. Telekom, Datev e.G.). Weitere Anbieter unter www.bundesnetzagentur.de. 
Rechtssicherheitsgründen ${ }^{149}$ die Identifizierung des Gläubigers ermöglichen und dessen unbedingten Willen zum Ausdruck bringen, die volle Verantwortung für den Inhalt seiner verfahrensrechtlich wirklich gewollten Anmeldung zu übernehmen, sondern im Fall des elektronischen Dokuments auch im Interesse des Absenders eine Manipulation am Dokument im elektronischen Verkehr verhindern. Dass es sich beim Insolvenzverfahren „lediglich“ um ein gerichtlich geführtes Verfahren handelt, kann dem nicht entgegenstehen.

Fehlt somit bei der Anmeldung die Unterschrift des Anmeldenden, ist die Forderungsanmeldung grundsätzlich unzulässig. ${ }^{150}$

\section{c) Vertretung}

Die Anmeldung kann weiterhin durch den Insolvenzgläubiger selbst, bei dessen Prozessunfähigkeit (§§ 50ff. ZPO i.V.m. § 4 InsO) durch dessen gesetzlichen Vertreter oder auch einen gewillkürten Vertreter erfolgen. § $174 \quad$ I 3 InsO stellt inzwischen klar, dass zur Vertretung des Insolvenzgläubigers im Rahmen des Anmeldeverfahrens auch Personen befugt sind, die Inkassodienstleistungen erbringen (registrierte Personen nach $\S 10$ Abs. 1 Satz 1 Nr. 1 des Rechtsdienstleistungsgesetzes). Die Vorlage einer schriftlichen Vollmacht ist dann jedoch erforderlich, ${ }^{151}$ wobei eine vorläufige Zulassung der Anmeldung gem. § 89 I ZPO i.V.m. § 4 InsO bei späterer Nachreichung der Vollmacht möglich ist. Eine Ausnahme gilt nach § 88 II ZPO nur für Rechtsanwälte, wobei der Mangel der Vollmacht vom Insolvenzverwalter oder einem Insolvenzgläubiger gerügt werden kann. ${ }^{152}$

BGHZ 37, 156 (157); 75, 340 (349); 97, 283 (285).

Siehe auch unter D.IV.5. (S.53ff.).

LG München II ZIP 1992, 789 (789); MüKo-InsO/Nowak, § 174 Rn.4; Gottwald/Eickmann, § 63 Rn.4; Uhlenbruck/Uhlenbruck, § 174 Rn.13 m.w.N.

152 MüKo-InsO/Nowak, § 174 Rn.4; Gottwald/Eickmann, $\S 63 \quad$ Rn.4; Uhlenbruck/Uhlenbruck, § 174 Rn.13 m.w.N. 


\section{d) Zusammengefasste Anmeldungen}

Weiterhin sind auch Sammelanmeldungen oder sog. „Poolanmeldungen“ grundsätzlich zulässig. ${ }^{153}$ Bei diesen Anmeldungen handelt es sich um eine einheitlich zusammengefasste Anmeldung mehrer Forderungen eines oder mehrer Berechtigten. Insbesondere im täglichen Geschäftsverkehr entsteht zwischen denselben Geschäftspartnern eine Vielzahl an Forderungen, die zur besseren Übersicht und Bearbeitung und damit zur Verfahrensvereinfachung in einer Anmeldung aufgeführt werden können. Voraussetzung für die Zulässigkeit von Sammel- und Poolanmeldungen ${ }^{154}$ ist aber, dass sich aus der Anmeldung die einzelnen Forderungen der Anspruchsberechtigten individualisieren lassen, damit die Möglichkeit des Insolvenzverwalters und der Insolvenzgläubiger einzelne Insolvenzforderungen $\mathrm{zu}$ prüfen und gegebenenfalls $\mathrm{zu}$ bestreiten, nicht eingeschränkt oder ausgeschlossen wird. ${ }^{155}$ Denn letztlich handelt es sich hierbei auch nur um die Anmeldung einzelner Insolvenzforderungen, die zur Verfahrensvereinfachung in einer Anmeldung zusammengefasst werden. Die einzelnen Insolvenzforderungen in der Anmeldung zu addieren und nur einen Gesamtbetrag ${ }^{156}$ mit einer Aufstellung der hieran beteiligten Insolvenzgläubiger anzumelden ist daher unzulässig. ${ }^{157}$

Kommt es zu Doppelanmeldungen, wird also ein und dieselbe Insolvenzforderung von mehreren Personen angemeldet, hat der Insolvenzverwalter in der Bemerkungsspalte der Tabelle auf die Doppelanmeldung hinzuweisen. ${ }^{158}$ Erkennt der Verwalter die Forderung als solche sodann im Prüfungstermin ( $§ 176$ InsO) an, so geschieht dies zweckmäßig „unter dem Vorbehalt, dass sie demjenigen zusteht, der im

BGH NJW 1998, 2592 (2597); MüKo-InsO/Nowak, § 174 Rn.12; FK/Kießner, § 174 Rn.30; Uhlenbruck/Uhlenbruck, § 174 Rn.15; Gottwald/Eickmann, § 63 Rn.5.

$154 \mathrm{Zu}$ allgemeinen Problematiken bei Poolverträgen siehe ausführlich bei Ripka, Poolverträge.

155 BGH ZInsO 2009, 381 (381f.). Siehe auch nachfolgend unter D.IV.3.a) (S.44ff.).

56 RGZ 39, 45 (45ff.).

157 BAG ZIP 1986, 518 (518).

158 BGH NJW 1979, 810 (810f.); NJW 1997, 1014 (1015); Gottwald/Eickmann, § 63 Rn.2; MüKo-InsO/Nowak, § 174 Rn.13 m.w.N. 
Streit über die Inhaberschaft obsiegt“ (sog. Prätendentenstreit). Dies ist entsprechend als Bemerkung in die Tabelle aufzunehmen. ${ }^{159}$

\section{Der wesentliche Inhalt der Forderungsanmeldung}

Außer der als selbstverständlich vorauszusetzenden eindeutigen Bezeichnung des Verfahrens, auf das sich die Anmeldung bezieht und der ebenfalls eindeutigen Bezeichnung des Insolvenzgläubigers sind in der Anmeldung gem. $§ 174$ II InsO weiterhin der Grund und der Betrag der Insolvenzforderung anzugeben, sowie die Tatsachen, aus denen sich nach Einschätzungen des Gläubigers ergibt, dass ihr eine vorsätzlich begangene unerlaubte Handlung des Schuldners zugrunde liegt.

\section{a) Grund der Insolvenzforderung}

Hinsichtlich des Grundes hat der Insolvenzgläubiger - ähnlich wie beim Klageantrag hinsichtlich des Streitgegenstandes - die Tatsachen vorzutragen, aus denen seine Forderung resultiert, also die Darstellung des Lebenssachverhaltes, aus dem sich die Berechtigung zweifelsfrei konkret, individuell und schlüssig ergibt, wobei eine rechtliche Einordnung der Tatsachen nicht erforderlich ist. ${ }^{160}$ Die Angabe des Grundes dient den Verfahrensbeteiligten zur Überprüfung der Verfahrensberechtigung des Anmeldenden, so dass hierfür die gleichen vom BGH für den Zivilprozess entwickelten Substanziierungsgrundsätze gelten. ${ }^{161}$ Die Darstellung hat daher so zu erfolgen, dass es dem Insolvenzverwalter und den Insolvenzgläubigern möglich ist, im Einzelnen über Berechtigung oder

159 Quoten, die bei einer Auszahlung auf die angemeldete Insolvenzforderung entfallen, kann der Insolvenzverwalter unter den Voraussetzungen des § 372 S.2 BGB unter Nennung aller eingetragenen Gläubiger und zugunsten des Obsiegenden von ihnen befreiend hinterlegen. - BGH NJW 1997, 1014 (1015); Müko-InsO/Nowak, § 177 Rn.12 m.w.N.

160 RGZ 93, 13 (14); HHK/Preß/Henningsmeier, § 174 Rn.15; FK/Kießner, § 174 Rn.14; HK/Irschlinger, § 174 Rn.10; Graf-Schlicker/Graf-Schlicker, § 174 Rn.15; MüKoInsO/Nowak, § 174 Rn.10. Vgl. auch BGH ZInsO 2009, 381 (381) m.w.N.

161 BGH NJW 1984, 2888 (2888ff.); siehe auch MüKo-InsO/Nowak, § 174 Rn.10 m.w.N. 
Nichtberechtigung der Forderung zu entscheiden. ${ }^{162}$ Wird eine Forderung aus fremdem Recht geltend gemacht, bedarf es daher näheren Sachvortrags zum Rechtserwerb des Gläubigers. Ebenso ist zum Verpflichtungsgrund des Schuldners vorzutragen, wenn sich die Forderung ursprünglich nicht gegen ihn, sondern gegen einen Dritten richtete. ${ }^{163}$

Bei Insolvenzforderungen, die auf einer vorsätzlich begangenen unerlaubten Handlung des Schuldners beruhen, wird im Rahmen der Forderungsanmeldung jedoch eine zusätzliche (rechtliche) Bewertung des Insolvenzgläubigers verlangt, wodurch der Schuldner nach Einschätzung des Insolvenzgläubigers eine unerlaubte Handlung vorsätzlich begangen hat. ${ }^{164}$ Diese zusätzliche Angabe ist jedoch keine Wirksamkeitsvoraussetzung für die Anmeldung, weil die Frage der unerlaubt begangenen Handlung des Schuldners nicht die Insolvenzmasse, sondern nur die Vollstreckungsmöglichkeit nach der Aufhebung des Insolvenzverfahrens und der Wohlverhaltensphase betrifft. ${ }^{165}$ Der Schuldner soll durch die Angabe lediglich bereits bei der Anmeldung durch den angegebenen Rechtsgrund davor gewarnt werden, dass der anmeldende Gläubiger gem. § 302 Nr.1 InsO von der Erteilung einer Restschuldbefreiung mit seiner Forderung nicht berührt wird und dem Schuldner rechtzeitig die Möglichkeit verschaffen, die Gefahr einer nicht vollständigen Restschuldbefreiung abschätzen zu können. Denn für den Schuldner würde es eine erhebliche Härte bedeuten, wenn er erst nach erfolgtem Durchlaufen der Wohlverhaltensphase erfahren würde, dass eine derartige Insolvenzforderung nicht von der Restschuldbefreiung erfasst wäre. ${ }^{166}$ Weist der Insolvenzgläubiger andererseits bei der Anmeldung seiner Forderung nicht darauf hin, ${ }^{167}$ dass die Insolvenzforderung nach seiner Einschätzung auf einer vorsätzlich begangenen unerlaubten

162 Durch diese Anforderung an eine Prüfungsmöglichkeit der angemeldeten Insolvenzforderung handelt es sich hier um eine deutliches Mehr im Vergleich zu den Anforderungen eines Mahnbescheids, bei dem eine hinreichende Individualisierung ausreicht, so dass die Forderung von anderen Forderungen unterschieden werden kann. Das Verfahren steht in diesem Punkt der Klageschrift (§ 253 ZPO) deutlich näher als dem Antrag auf Erlass eines Mahnbescheids. - Siehe nur Zöller/Vollkommer, § 690 Rn.14 und § 253 Rn.12a jeweils m.w.N. BGH ZInsO 2009, 381 (381f.).

164 LG Bochum, Beschl. v. 6.8.2004 - 10 T 50/04 (bisher wohl unveröffentlicht); GrafSchlicker/Graf-Schlicker, \& 174 Rn.16; Braun/Specovius, \& 174 Rn.39ff.; Kehe/Meyer/Schmerbach, ZinsO 2002, 615 (617). Siehe auch E.I.4.c)cc) (S.148ff.).

166 Begr. zu Nr.12 (§ 174 InsO) RegE, BT-Drucks. 14/5680, S.27.

167 Zur Ergänzung der Anmeldung siehe unter D.IV.7. (S.72ff.). 
Handlung beruht und wird diese dann ohne diesen Zusatz zur Tabelle festgestellt, so kann sich der Gläubiger nach Beendigung des Schlusstermins ${ }^{168}$ auf einen Ausschluss seiner Forderung von der Restschuldbefreiung nicht mehr berufen, und seine Insolvenzforderung wird von der Restschuldbefreiung erfasst (vgl. § 302 Nr.1 InsO). ${ }^{169}$ Der frühzeitige Hinweis auf eine vom Schuldner gestellte Restschuldbefreiung erfolgt für die Insolvenzgläubiger gem. § 27 II Nr.4 InsO bereits mit dem Eröffnungsbeschluss. ${ }^{170}$ Inwieweit eine nachträgliche Feststellung des Grundes der vorsätzlich begangenen unerlaubten Handlung möglich ist, wird an anderer Stelle noch zu behandeln sein. ${ }^{171}$

\section{b) Betrag der Insolvenzforderung}

Die Angabe des Insolvenzforderungsbetrages in Euro ist ebenso wie der allgemeine Forderungsgrund Wirksamkeitsvoraussetzung für die Anmeldung (§ 174 II InsO). ${ }^{172}$

Da die insolvenzrechtliche gleichmäßige Befriedigung der Insolvenzgläubiger aus der Masse nur durchführbar ist, wenn sich die Forderungen für die Berechnung der Quote eignen, ${ }^{173}$ sind Forderungen, die nicht auf Geld gerichtet sind oder deren Geldbetrag unbestimmt ist (z.B. Ansprüche auf Nachbesserung, Rückgewähr von Gegenständen), mit dem gemeinen Wert bzw. Kurswert ${ }^{174}$ geltend zu machen, der für die Zeit der Eröffnung des Insolvenzverfahrens geschätzt werden kann (§ 45 S.1 InsO).

Für wiederkehrende Leistungen (z.B. Forderungen aus einem Leibrentenvertrag, Rentenansprüche der Angestellten, Rentenansprüche aus unerlaubter Handlung nach § 843 BGB) bestimmt $\S 46$ InsO eine Kapitalisierung der Insolvenzansprüche.

\footnotetext{
Zur letzten Anmeldungsmöglichkeit siehe unter E.II.3.a) (S. 192ff.).

Begr. zu Nr.12 (§ 174 InsO) RegE, BT-Drucks. 14/5680, S.28.

Siehe auch schon D.III. (S.33ff.).

Siehe dazu unter D.IV.7. (S.72ff.).

72 Kübler/Prütting/Bork/Pape, § 174 Rn.27; Braun/Specovius, § 174 Rn.27.; MüKoInsO/Nowak, § 174 Rn.15 m.w.N.

173 BGH ZVI 2003, 2379 (2381); siehe auch schon oben C.II. (S.16ff.).

174 Ein besonderes Affektionsinteresse des Gläubigers ist unbeachtlich - GrafSchlicker/Mäusezahl, § 45 Rn.3; Kübler/Prütting/Bork/Holzer, § 45 Rn.6; MüKoInsO/Lwowski/Bitter, § 45 Rn.25 m.w.N.
} 


\section{c) Absonderungsberechtigte (Insolvenz-)Gläubiger}

Das Recht zur Verwertung unbeweglicher (§ 165 InsO) und beweglicher Gegenstände ( $§ 166$ InsO), an denen ein Absonderungsrecht besteht, übt regelmäßig der Insolvenzverwalter aus. Zu einer Verwertung durch die absonderungsberechtigten Gläubiger ( $\S 170$ II, 172 I, II InsO) kommt es hingegen selten. Aufgrund der kurzfristigen Verwertung dieser Gegenstände (§ 159 InsO) kann in den meisten Fällen nicht der Erlös erreicht werden, den der absonderungsberechtigte Gläubiger als Sicherheit an dem verwerteten Gegenstand hatte. ${ }^{175}$ Zudem mindert sich der Verwertungserlös zusätzlich durch Abzug und Abführung der Kosten für die Feststellung und Verwertung des Gegenstandes in die Insolvenzmasse (§§ 170, 171 InsO).

Daher können absonderungsberechtigte Gläubiger (§§ 49ff. InsO), ${ }^{176}$ deren Absonderungsrecht eine persönliche Forderung gegen den Schuldner sichert und die daher zugleich auch Insolvenzgläubiger sind, ${ }^{177}$ ihre Insolvenzforderung zunächst in voller Höhe zur Tabelle anmelden. ${ }^{178}$ Ihr Charakter als Ausfallforderung wirkt sich erst im Verteilungsverfahren aus (vgl. § 190 InsO), indem der Gläubiger gem. § 52 S.2 InsO nur insoweit anteilmäßig Befriedigung erlangen kann, als er mit seiner persönlichen Forderung ausgefallen ist. Wenn der Gläubiger auf sein Absonderungsrecht jedoch verzichtet, wird er mit seiner vollen persönlichen Forderung im Verteilungsverfahren berücksichtigt. ${ }^{179}$ Verzicht bedeutet dabei die endgültige und vorbehaltslose Aufgabe des die

175 Hieran ändert auch die dem (fachkundigeren) absonderungsberechtigten Gläubiger gegebene Gelegenheit, den Insolvenzverwalter auf eine günstigere Verwertungsmöglichkeit hinzuweisen (§ 168 I InsO), oftmals nichts.

Die grundsätzliche Einbeziehung der absonderungsberechtigten Gläubiger - unabhängig von einer persönlichen Forderung gegen den Schuldner - in das Insolvenzverfahren beschränkt sich im Wesentlichen auf den Zeitpunkt und die Art der Verwertung der mit Absonderungsrechten belasteten Gegenstände und die Möglichkeit, diese Rechte in einem Insolvenzplan umzugestalten (vgl. § 223 InsO). - Vgl. Begr. zum 5.Teil (§§ 174ff. InsO) RegE, BT-Drucks. 12/2443, S.183f.

177 Siehe bereits unter C.II. (S.16ff.).

178 RGZ 78, 71 (75); 139, 83 (85); 155, 95 (101); HK/Eickmann, § 52 Rn.1; KS/Eckardt, S.768 Rn.47; Klasmeyer/Elsner, S.303; MüKo-InsO/Ganter, $\S 52$ Rn.17; Uhlenbruck/Uhlenbruck, § 52 Rn.2 m.w.N.

179 OLG Hamm ZIP 1994, 1373 (1375); Uhlenbruck/Uhlenbruck, § 52 Rn.16 m.w.N. 
Absonderung begründenden Rechts über das konkrete Verfahren hinaus, ${ }^{180}$ so dass der Gegenstand der Absonderung für die Masse unbelastet frei wird und verwertet werden kann. ${ }^{181} \mathrm{Er}$ kommt insbesondere dann in Betracht, wenn der Sicherungsgegenstand wirtschaftlich nicht sinnvoll ist. Der Verzicht kann, sofern eine bestimmte Form nicht zu wahren ist, vor Verfahrenseröffnung gegenüber dem Schuldner oder nach Eröffnung gegenüber dem Insolvenzverwalter als alleinigem Verwaltungs- und Verfügungsberechtigten ( $§ 80$ I InsO) erklärt werden. ${ }^{182}$ Dies kann auch durch schlüssiges Verhalten des Absonderungsberechtigten geschehen, wobei die vorbehaltlose Anmeldung der Forderung oder das bloße Unterlassen der Ausübung des Absonderungsrechts - die grundsätzlich der Disposition des Gläubigers unterliegt ${ }^{183}$ - keinen automatischen Verzicht darstellt. ${ }^{184}$ Es bedarf somit stets der Einzelfallbetrachtung.

\section{d) Nachrangige Insolvenzgläubiger}

Soweit nachrangige Insolvenzgläubiger zur Anmeldung ihrer Forderungen aufgefordert werden, ist gem. § 174 III 2 InsO auf deren Nachrang hinzuweisen und die dem Gläubiger zustehende Rangstelle zu bezeichnen. Fehlt der Hinweis, ist die Anmeldung jedoch gleichwohl zulässig. ${ }^{185}$

\section{e) Insolvenzgläubiger aus anderen EU-Mitgliedstaaten}

Für den Inhalt der Forderungsanmeldung eines Insolvenzgläubigers eines EU-Mitgliedstaates gilt zunächst Art. 41 EuInsVO. Demnach hat der Insolvenzgläubiger die Art, den Entstehungszeitpunkt und den Betrag der

\footnotetext{
180 FK/Joneleit/Imberger, $\S 52$ Rn.4; Nerlich/Römermann/Andres, $\S 52$ Rn.8; HK/Eickmann, § 52 Rn.7; Kübler/Prütting/Bork/Prütting, § 52 Rn.5; MüKoInsO/Ganter, § 52 Rn.39ff.; Hess, InsO, § 52 Rn.29; Kuhn/Uhlenbruck, § 64 KO Rn.13 m.w.N.

181 Auch ein Teilverzicht ist z.B. im Planverfahren gem. §§ 217ff. InsO möglich.

182 MüKo-InsO/Ganter, Vor §§ 49-52 Rn.123ff.; Uhlenbruck/Uhlenbruck, § 52 Rn.16 m.w.N.

183 Siehe bereits unter D.II. (S.30ff.).

184 RGZ 16, 32 (36); 16, 68 (70); OLG Hamm ZIP 1994, 1373 (1375); Gottwald/Gottwald, $\S \quad 42$ Rn.64f.; MüKo-InsO/Ganter, $\S 52$ Rn.39ff.; Kuhn/Uhlenbruck, § 64 KO Rn.13a m.w.N.

185 Siehe auch D.IV.5. (S.53ff.).
} 
Forderung mitzuteilen und anzugeben, ob er für die Forderung ein Vorrecht, eine dingliche Sicherheit oder einen Eigentumsvorbehalt beansprucht und welche Vermögenswerte Gegenstand seiner Sicherheit sind. Diese inhaltlichen Anforderungen stehen bereits weitgehend mit denen des $\S 174$ InsO (Grund und Betrag) im Einklang und werden durch einen an alle bekannten Insolvenzgläubiger aus EU-Mitgliedsstaaten individuell zuzustellenden Informationsvermerk im Rahmen eines Formblattes gem. Artt. 40 II, 42 I 2 EuInsVO („Formblatt Europäische Insolvenzordnung“) ${ }^{186}$ mit den sonstigen Regelungen des $\S 174$ InsO ergänzt, so dass sich durch Art. 41 EuInsVO für die inhaltlichen Voraussetzungen der Insolvenzforderungsanmeldung letztlich keine Veränderungen ergeben.

\section{Beizufügende Urkunden und sonstige Beweisstücke}

\section{a) Urkunden und Beweisstücke}

Den Anmeldungen sollen die Urkunden, aus denen sich die Insolvenzforderungen ergeben, in Abdruck beigefügt werden (§ 174 I 2 InsO).

Die Urkunden sollen - hinsichtlich der Substantiierungslast des Anmeldenden ${ }^{187}$ - dem Insolvenzverwalter und den Insolvenzgläubigern ermöglichen, die Berechtigung der ordnungsgemäß angemeldeten Insolvenzforderung auf Teilnahme am Verfahren zu prüfen. Diese materiellrechtliche Prüfung der Insolvenzforderung erfolgt bereits im Vorfeld des Prüfungstermins durch die ermöglichte Einsicht ${ }^{188}$ der beim Insolvenzgericht mit der Tabelle niedergelegten Urkunden/Unterlagen. ${ }^{189}$ Als Urkunde kommen hier jegliche Schriftstücke in Betracht, mit denen der Beweis für das Bestehen der Insolvenzforderung zu führen ist (z.B. Titel, Verträge, Rechnungen, etc.). Während nach § 138 S.3 KO die urkundlichen Beweisstücke der Anmeldung beizufügen waren, handelt es

\footnotetext{
186 In den jeweiligen EU-Amtssprachen beim Bundesministerium der Justiz unter http://www.bmj.bund.de/enid/Insolvenzrecht/Formblatt_Europaeische_Insolvenzvero rdnung ty.html abrufbar; Stand: Juni 2009. 
sich bei § 174 I 2 InsO lediglich noch um ein Sollvorschrift. Daher ist im Gegensatz zur Angabe des Anspruchsgrundes und dessen Höhe, die Beifügung von Urkunden und sonstigen Beweisstücke keine zwingende Voraussetzung für eine ordnungsgemäße Anmeldung der Insolvenzforderung. ${ }^{190}$ Für die elektronische Anmeldung bestimmt das Gesetz gem. § 174 IV 2 InsO ausdrücklich, dass die Urkunden unverzüglich nachzureichen sind. Auch Art. 41 EuInsVO fordert vom europäischen Gläubiger bereits mit der Anmeldung auch die Übersendung einer Kopie der vorhandenen Belege.

Unterlässt der anmeldende Gläubiger trotz Aufforderung des Insolvenzverwalters ${ }^{191}$ überhaupt irgendwelche Beweisstücke einzureichen, setzt er sich dem Risiko aus, dass sein Anspruch zunächst bestritten und erst im Feststellungsprozess nach Vorlage der Beweisstücke sofort anerkannt wird mit der Folge, dass der Anmeldende die Kosten des Feststellungsstreits zu tragen hat. ${ }^{192}$

\section{b) Vorlage von Originalurkunden}

Fraglich ist hinsichtlich § 178 II 3 InsO, inwieweit die Vorlage von Originalurkunden im Verfahren notwendig ist, um die Insolvenzforderung im Prüfungstermin (§ 176 InsO) feststellen zu können. Denn die Vorlage von Originalen verlangt das Gesetz im Verfahrensstadium der Anmeldung gerade nicht (vgl. § 174 I 2 InsO: „Abdruck“). ${ }^{193}$ Nach § 178 II 3 InsO ist jedoch auf Wechseln und sonstigen Schuldurkunden vom Urkundsbeamten der Geschäftsstelle die Feststellung der zugrundeliegenden Forderung zur Insolvenztabelle $\mathrm{zu}$ vermerken. Hieraus wird teilweise die Ansicht

\footnotetext{
$190 \S 139$ S.3 KO (entspricht § 174 I 2 InsO) ist lediglich eine Ordnungsvorschrift. - RGZ 85, 64 (68); BGH ZIP 2006, 192 (193); OLG Hamburg KTS 1975, 42 (43); Kübler/Prütting/Bork/Pape, § 174 Rn.28; Mohrbutter/Ringstmeier/Ernestus, § 11 Rn.8; Braun/Specovius, § 174 Rn.21f.; Uhlenbruck/Uhlenbruck, § 174 Rn.20 m.w.N.

191 Dazu auch unter D.IV.5.b) (S.56ff.).

192 OLG Hamburg KTS 1975, 42 (43); OLG Hamm ZInsO 1999, 352 (352); LG Aurich, ZinsO 2006, 102 (102); FK/Kießner § 174 Rn.18ff.; MüKo-InsO/Nowak, $\S 174$ Rn.23; Uhlenbruck/Uhlenbruck, § 174 Rn.20 m.w.N.

193 BGH ZIP 2006, 192 (193); MüKo-InsO/Nowak, § 174 Rn.23; Graf-Schlicker/GrafSchlicker, § 174 Rn.12; Kübler/Prütting/Bork/Pape, § 174 Rn.28.
} 
gefolgert, ${ }^{194}$ dass der Gläubiger einer Forderung, für die z.B. ein Vollstreckungstitel, Wechsel oder eine sonstige Schuldurkunde ausgestellt ist, spätestens im Prüfungstermin die Originalurkunde vorweisen muss. Dies wird damit begründet, dass die betreffende Forderung zur Vermeidung ihrer Doppeltitulierung - zum einen durch den Originaltitel, zum anderen durch vollstreckbaren Auszug aus der Tabelle, der gem. § 201 II 3 InsO auf Antrag nach Aufhebung des Insolvenzverfahrens (§ 200 I InsO) erteilt wird - nicht festgestellt werden dürfe und daher durch den Insolvenzverwalter bis zur Vorlage des Originaltitels zu bestreiten sei. Dies hätte jedoch zur Folge, dass der Insolvenzgläubiger, obwohl seine Insolvenzforderung grundsätzlich am Verfahren teilnahmeberechtigt wäre, in einen u.U. langwierigen Feststellungsprozess gezwungen würde (§ 179 I InsO). Zudem wirkt sich der Widerspruch unmittelbar auf die Verfahrensrechte des Insolvenzgläubiger aus, indem sich z.B. sein Stimmrecht nunmehr nach dem Verfahren des § 77 II InsO bestimmt. ${ }^{195}$ Unabhängig von diesen schwerwiegenden verfahrensrechtlichen Folgen für den Insolvenzgläubiger eines hier geforderten Widerspruchs durch den Insolvenzverwalter, kann dieser Ansicht dagegen schon aus anderen Gründen nicht gefolgt werden. Denn ebenso wie der vom Wortlaut identische frühere $\S 145$ I 2 KO dient auch der jetzige $\S 178$ II 3 InsO nach der Intention des Gesetzgebers in erster Linie den Interessen des anmeldenden Gläubigers und soll ihm die Übertragung verbriefter Forderungen erleichtern. ${ }^{196}$ Der neue Gläubiger hat aufgrund des Vermerks die Gewissheit, dass die Insolvenzforderung nicht bestritten ist und an der Verteilung teilnimmt. Außerdem kann er aus der Urkunde unmittelbar ersehen, dass auf die Forderung nur eine Quote gezahlt wird. Darüber hinaus ergibt sich aus dem Wortlaut des $\S 178$ II 3 InsO zwar auch, dass ein Insolvenzgläubiger neben einer vollstreckbaren Ausfertigung aus der Tabelle (§ 201 II 3 InsO) nicht zugleich über weitere Urkunden verfügen soll, aus denen er wegen seiner im Verteilungsverfahren nicht voll befriedigten Insolvenzforderung die Einzelvollstreckung betreiben

194 AG Mönchengladbach ZinsO 2003, 291 (291); HK/Irschlinger, § 174 Rn.14, § 178 Rn.4a; Merkle, Rpfleger 2001, 157 (165); grundsätzlich wohl auch Uhlenbruck/Uhlenbruck, § 174 Rn.20; Nerlich/Römermann/Becker, § 174 Rn.17. Dazu ausführlich unter D.VI.2. (S.98ff.).

196 Vgl. KO-Motive, S.363 = Hahn, S.327; auch Jaeger/Weber, KO, § 145 Rn.2; Nerlich/Römermann/Becker, § 178 Rn.22; Hofer, Rpfleger 2007, 361 (362) m.w.N. 
könnte. ${ }^{197}$ Doch postuliert $\S 178$ II 3 InsO gerade keine Pflicht, entsprechende Originale überhaupt vorzulegen bzw. die Feststellung betroffener Forderungen von deren Vorlage abhängig zu machen. ${ }^{198}$ Daher kann auch ein Widerspruch allein unter Berufung auf $\S 178$ II 3 InsO im nachfolgenden Feststellungsprozess nach den $\S \S 179 f f$. InsO keinen Erfolg haben. ${ }^{199}$ Der Forderungsnachweis im Feststellungsrechtsstreit auf Feststellung einer titulierten Forderung zur Insolvenztabelle kann nämlich auch hier nicht nur im Wege des Urkundsbeweises, sondern mit sämtlichen nach der Zivilprozessordnung zulässigen Beweismitteln geführt werden. So genügt etwa nach den $\S \S 420,435$ ZPO bei öffentlichen Urkunden - z.B. der Vollstreckungsbescheid (§ 699 ZPO) - die Vorlage einer öffentlich beglaubigten Abschrift, wenn nicht das Gericht aus besonderem Anlass die Vorlage der Unterschrift anordnet. Dies zeigt, dass es auch bei der zivilprozessualen Feststellung einer titulierten Forderung nicht notwendig der Vorlage des Originaltitels bedarf. ${ }^{200}$ Eine Doppeltitulierung kann zudem stets dadurch vermieden werden, dass - in der Praxis schon unproblematisch gängig ${ }^{201}$ - das Insolvenzgericht die spätere Erteilung des vollstreckbaren Tabellenauszugs von der Vorlage der Originalurkunde zur „Entwertung“ abhängig macht. ${ }^{202}$ Diese Verfahrensweise lässt sich durchaus auf den Wortlaut „ist...die Feststellung zu vermerken“ des $§ 178$ II 3 InsO stützen. Selbst wenn dies unterbleiben sollte und der Gläubiger aus dem früheren Titel die Vollstreckung betreibt, obwohl über den deckungsgleichen Anspruch ein vollstreckbarer Tabellenauszug vorliegt, kann sich der Schuldner hiergegen noch mit dem jeweils statthaften Rechtsbehelf der §§ 766ff. ZPO erwehren.

\footnotetext{
Siehe dazu unter E.III.5.b) (S.224ff.).

198 Hierfür findet sich keine Stütze im Gesetz. - RGZ LZ 1913, 630 (632); BGH ZIP 2006, 192 (193); Graf-Schlicker/Graf-Schlicker, § 178 Rn.8; MüKo-InsO/Nowak, $\S 174$ Rn.23; FK/Kießner, § 178 Rn.17; Köster/Ahrendt, EWiR 2006, 177 (178). BGH ZIP 2006, 192 (193).

200 BGH ZIP 2006, 192 (194); Köster/Ahrendt, EWiR 2006, 177 (178); Smid, JurisPRInsR 2006, Anm. 3.

201 AG Mönchengladbach ZinsO 2003, 291 (291); Kaiser/Crämer, InVo 2001, 153 (154).

202 So auch BGH ZIP 2006, 192 (193); Köster/Ahrendt, EWiR 2006, 177 (178); Braun/Specovius, $\S 178$ Rn.17; Graf-Schlicker/Graf-Schlicker, $\S 178$ Rn.8; HK/Preß/Henningsmeier, § 178 Rn.17.
} 
Der zwingenden Vorlage des Originaltitels einer angemeldeten und bereits titulierten Insolvenzforderung bedarf es zu ihrer Feststellung im Insolvenzverfahren daher nicht.

\section{Unzulässige Anmeldungen}

Im Folgenden soll (a) auf formell unzulässige Anmeldungen eingegangen und die Frage hinsichtlich einer notwendigen Befugnis ihrer (Vor-)Prüfung und Zurückweisung innerhalb des Anmeldeverfahrens aufgeworfen werden. Die Problematik, wem dieses Recht in welchem Umfang zusteht, soll (b) unter notwendiger Abgrenzung zur materiellrechtlichen Prüfungskompetenz im Insolvenzverfahren und (c) mit Blick auf die Kompetenzverteilung unter der vormaligen Konkursordnung (d) einer Lösung unter der Insolvenzordnung zugeführt und (e) deren Folgen für den Zurückgewiesenen und dessen Möglichkeiten aufgezeigt und erörtert werden.

\section{a) Formell unzulässige Anmeldungen}

Wie beim Klageantrag oder anderen Anträge zur Durchsetzung von Interessen müssen vom Antragenden stets die für das entsprechende Verfahren gesetzlich festgelegten formellen Voraussetzungen für die Zulassung zu einer Entscheidung in der angetragenen Sache erfüllt werden. Dadurch wird zum einen die Gleichbehandlung aller Antragenden hinsichtlich des Zugangs zu einer Entscheidung gewährleistet, zum anderen legen die $\mathrm{zu}$ erfüllenden formellen Voraussetzungen das Mindestmaß der vom Antragenden bereitzustellenden Informationen fest, die zur Teilnahme am Verfahren und im weiteren zur Beurteilung der Erfolgsaussichten in der angetragenen Sache benötigt werden.

Für das Insolvenzverfahren sind diese formellen Voraussetzungen - wie bereits besprochen ${ }^{203}$ - in $\S 174$ InsO festgelegt. Die Anmeldung des Insolvenzgläubigers dient im Rahmen des Insolvenzverfahrens dazu, für

203 Siehe bereits D.IV.2. und 3. (S.36ff., 44ff.). 
eine Teilnahme am Insolvenzverfahren die notwendigen Informationen über seine Insolvenzforderung(en) bereitzustellen, um über deren Berechtigung auf weitere Teilnahme am Verfahren durch die übrigen Verfahrensteilnehmer entscheiden zu können. Entspricht eine Anmeldung diesen Voraussetzungen nicht, so kann im weiteren Verfahren auch keine ausführliche Beurteilung über deren Berechtigung erfolgen, so dass die Anmeldung/Insolvenzforderung nicht zum Insolvenzverfahren zugelassen werden kann. Weist eine Forderungsanmeldung formelle Mängel auf, so ist jedoch zu unterscheiden, ob es sich um wesentliche Mängel handelt, die die Forderungsanmeldung schlechthin formell unzulässig machen und sie daher den Voraussetzungen zur Teilnahme am Verfahren nicht genügt, oder um sonstige Mängel, die die formelle Zulässigkeit der Anmeldung an sich nicht berühren und daher einer Teilnahme nicht entgegenstehen.

Eine Anmeldung ist grundsätzlich unzulässig, wenn sie nicht den angesprochen Erfordernissen an Form ${ }^{204}$ und Inhalt ${ }^{205}$ der Anmeldung entspricht, da die mit der Anmeldung geltend gemachte Insolvenzforderung im weiteren Verfahren schon gar nicht prüfungs- und damit teilnahmefähig wäre. Dies ist insbesondere dann der Fall, wenn die Höhe der Forderung in fremder Währung, der den Schuldgrund bildende Sachverhalt für die Forderung nicht angegeben wurde, oder schlicht die verbindliche Unterschrift fehlt. Unzulässige Anmeldungen können jedoch grundsätzlich im Zeitpunkt der Behebung ihrer Mängel zulässig werden, indem z.B. eine fehlende Unterschrift nachgeholt ${ }^{206}$ wird. Hier ist dann danach zu unterscheiden, ob die Behebung innerhalb der Anmeldefrist (§ 28 I InsO) oder erst zu einem späteren Zeitpunkt und damit nach Ablauf

204 1. Die schriftliche Anmeldung in deutscher Sprache (§ 184 GVG) beim zuständigen Insolvenzverwalter. Wobei die sprachliche Ausnahmeregelung des Art. 42 II EuInsVO für Insolvenzgläubiger aus anderen EU-Mitgliedstaaten auch für Insolvenzgläubiger außerhalb der EU heranzuziehen ist. Der Insolvenzgläubiger hat in jedem Fall die Nachreichung einer deutschen Übersetzung der Anmeldung in angemessener Frist zu fordern (§ 42 II 3 EuInsVO). Siehe D.IV.2.a) (S.36ff.).

2. Eindeutige Bezeichnung des Verfahrens und des anmeldenden prozessfähigen Gläubigers und/oder seines ordnungsgemäßen Vertreters. Siehe D.IV.2.a) (S.36ff.).

3. Die Unterschrift des Anmeldenden ( $\S 130$ Nr.6 ZPO) oder alternativ zur Schriftform bei - durch den Insolvenzverwalter ausdrücklich zugelassener elektronischer Übermittlung (§ 174 IV InsO) - elektronischen Dokumenten in Form der qualifizierten elektronischen Signatur (§ 130a I 2 ZPO). Siehe D.IV.2.b) (S.38ff.).

205 1. Angabe des konkreten und individualisierten Lebenssachverhaltes (Grund), auf dem die Forderung beruhen soll. Siehe D.IV.3.a) (S.44ff.).

2. Angabe des Forderungsbetrages in Euro (Betrag). Siehe D.IV.3.b) (S.46f.).

206 Baumbach/Lauterbach/Albers/Hartmann, ZPO (2009), § 129 Rn.23 m.w.N. 
der Frist erfolgt. Für die Behebung innerhalb der Anmeldefrist ergeben sich keine Besonderheiten, so dass die nunmehr ordnungsgemäße Anmeldung am regulären Verfahren teilnimmt. Erfolgt die Behebung des Mangels jedoch nach Ablauf der Anmeldefrist, gilt sie als verspätet und unterfällt damit als nachträgliche Anmeldung dem § 177 InsO und dessen Folgen. ${ }^{207}$

Gleichermaßen unzulässig sind nachrangige Forderungen wenn sie als solche angemeldet werden, obwohl hierzu vom Insolvenzgericht keine Aufforderung gem. § 174 III InsO erteilt wurde. Wird auf den Nachrang jedoch nicht hingewiesen, sondern die Forderung schlicht als nichtnachrangige („vollwertige“) Insolvenzforderung angemeldet, so hindert dies zumindest ihre wirksame Anmeldung ${ }^{208}$ wegen ihrer zwischenzeitlichen Gleichstellung und generellen Einordnung als Insolvenzforderung ${ }^{209}$ nicht. $^{210}$

Hiervon zu unterscheiden sind zur Anmeldung vorliegende Masseforderungen $^{211}$ oder Aus- und Absonderungsrechte ${ }^{212}$, die schon gar nicht die Qualifikation einer Insolvenzforderung i.S.d. §§ 38, 39 InsO erfüllen. ${ }^{213}$ Solche Anmeldungen sind grundsätzlich unzulässig.

Fraglich ist jedoch, wer die Prüfung der formellen Zulässigkeit der Forderungsanmeldungen vornimmt und wie unzulässige Anmeldungen im Verfahrensablauf zu behandeln sind. Schon im Rahmen der damaligen Konkursordnung wurde regelmäßig die Frage aufgeworfen, inwieweit und insbesondere von wem (Urkundsbeamter - Verwalter Konkursgericht/Rechtspfleger) Forderungen, die trotz ihrer Unzulässigkeit

Dazu unter E.II. (S.187ff.).

Siehe auch unter D.IV.5.a) (S.53ff.).

Siehe bereits unter C.III. (S.20ff.).

210 Hiervon geht auch der Gesetzgeber aus: „Wenn eine nachrangige Forderung angemeldet wird, ohne daß der Gläubiger dabei auf den Nachrang hinweist, gilt dies als Anmeldung einer nicht nachrangigen Forderung; die Folge wird sein, daß der [vollwertige] Rang bestritten wird.“ Begr. zu § 201 (§ 174 InsO) RegE, BT-Drucks. 12/2443, S.184; mittlerweile auch ganz herrschende Meinung: LG Waldshut-Tiengen ZIP 2005, 557 (557f.); Kübler/Prütting/Bork/Pape, § 174 Rn.23; Braun/Specovius, § 174 Rn.34; Uhlenbruck/Uhlenbruck, § 174 Rn.26; HK/Irschlinger, § 174 Rn.16; MüKo-InsO/Nowak, § 174 Rn.32; KS/Eckardt, S.751 Rn.15; Eckardt, ZIP 1993, 1765 (1767); differenzierend Bähr, InVo 1998, 205 (208).

211 RG JW 1905, 389 (390); BGHZ 113, 381 (382); BAG ZIP 1987, 1266 (1267) jeweils m.w.N.

212 RGZ 55, 157 (159); BGH WM 1974, 1218 (1218) jeweils m.w.N.

213 Siehe bereits unter C.II. (S.16ff.) mit entsprechender Besonderheit bei Absonderungsrechten, die unter Umständen gleichzeitig Insolvenzforderung sein können. 
dennoch zur Tabelle angemeldet werden, im Rahmen einer „Vorprüfung“ im Anmeldeverfahren als unzulässig zurückgewiesen werden dürfen und damit keine Aufnahme in die Tabelle erfahren. Die Problematik einer solchen Zurückweisungsbefugnis im Vorfeld des Prüfungstermins ist, wegen der nahezu unveränderten systematischen und inhaltlichen Übernahme der Konkursregelungen in die Insolvenzordnung, auch weiterhin gegeben. ${ }^{214}$

Die allgemeinen Zurückweisungsmöglichkeit ergibt sich dabei nicht nur aus der grundsätzlichen Prüfung und Einhaltung der insolvenzrechtlichen Zulassungsregeln der $\S \S 174 f f$. InsO zum Insolvenzverfahren, sondern indirekt auch aus der ausschließlichen Zuweisung der Insolvenzmasse an die Insolvenzgläubiger und dem eingangs bereits dargestellten ${ }^{215}$ Ziel der gleichmäßigen Befriedigung ihrer wirksam angemeldeten Insolvenzforderungen (§£ 38, 174 i.V.m. § 1 S.1 InsO). Um diesem Ziel jedoch gerecht werden zu können, dürfen nur zulässige Anmeldungen von Insolvenzforderungen am Verfahren beteiligt werden und entsprechenden Einfluss auf den Fortgang des Verfahrens haben. Zudem soll die Insolvenzmasse/ Quote nicht mit unzulässigen Forderungsanmeldungen zusätzlich belastet werden. Zu klären ist daher, zu welchem Zeitpunkt des Anmeldeverfahrens von wem solche Zurückweisungsentscheidungen getroffen werden können. ${ }^{216}$

Hierzu soll zunächst eine genaue Einordnung der formellen und der materiellen Prüfung der Forderungsanmeldung bzw. der in ihr enthaltenen Insolvenzforderung vorgenommen werden, um durch deren Abgrenzung zueinander Rückschlüsse auf eine formelle Prüfungs- und Zurückweisungskompetenz und deren Umfang ziehen zu können.

\section{b) Differenzierung formelle - materielle Prüfung der Anmeldung}

Zu unterscheiden ist grundsätzlich zwischen der Prüfung der rein formellen Anforderungen an die Forderungsanmeldung (Form und Inhalt) ${ }^{217}$

\footnotetext{
214 Siehe die jeweiligen Verweise auf die entsprechenden Paragraphen der Konkursordnung in den folgenden Fußnoten.

215 Siehe auch B. (S.4ff.) und C.I. (S.10ff.).

216 Siehe auch unter E.I.3. (S.137ff.).

217 Siehe zusammenfassend Fn.204 und 205 (S.54).
} 
einerseits, die zwingend für eine formell zulässige Forderungsanmeldung vorliegen müssen, und deren materiellen Prüfung andererseits, also der sachlich berechtigten Teilnahme am Anmelde- und Feststellungsverfahren und Anteilhabe an der Zuweisung der Insolvenzmasse durch tatsächliches Bestehen der angemeldeten Forderung und deren Qualifikation als Insolvenzforderung für deren Anerkennung und Feststellung zur Tabelle. Bei der formellen Prüfung geht es also um die Prüfung der bloßen Anmeldung hinsichtlich der bereits erörterten Einhaltung ihrer inhaltlichen Mindestangaben in einer bestimmten Form (vgl. § 174 InsO), wodurch die materiellrechtliche Prüfung der Insolvenzforderung im weiteren Verfahren überhaupt erst ermöglicht wird.

Eine materielle Zurückweisungsbefugnis sowohl unter der Konkursordnung als auch unter der jetzigen Insolvenzordnung ${ }^{218}$ steht ausschließlich den Verfahrensbeteiligten bzw. den Widerspruchsberechtigten $^{219}$ im gemeinschaftlichen Prüfungstermin zu. Durch die Zuweisung der Masse an die Gesamtheit der Insolvenzgläubiger sind nur diese - anstelle des Insolvenzschuldners - befugt, gegenseitig die konkurrierenden Insolvenzforderungen anzuerkennen oder zu bestreiten ${ }^{220}$ und damit zurückzuweisen. Das Widerspruchsrecht des Insolvenzverwalters findet dabei seine Rechtfertigung sachlich in seiner Amtsstellung, ${ }^{221}$ kraft deren er die Interessen der abwesenden Insolvenzgläubiger, des Schuldners und der sonstigen Betroffenen wahrzunehmen und die Verwaltungs- und Verfügungsbefugnis über die Insolvenzmasse ( $§ 80$ I InsO) hat, und tatsächlich in dessen häufig

218 Etwas anderes kann vergleichsweise aufgrund der fast identischen Systematik auch nicht der Gesamtvollstreckungsordnung oder der Vergleichsordnung entnommen werden. Vergleiche hierzu nur $\S 11$ GesO (siehe auch Hess/Binz/Wienberg, GesO, Einleitung Rn.36f.) und $\S \S$ 67, 70 i.V.m. 25 VglO (siehe auch BöhleStamschräder/Kilger, VglO, Einleitung IV.).

219 Siehe dazu unter D.VI.1. (S.94ff.) und E.I.4.a) (S.141f.).

220 Siehe schon C.I. (S.10ff.).

221 Zur Rechtsstellung des Insolvenzverwalters werden drei Theorien vertreten: die „Vertretertheorie“, nach der der Verwalter gesetzlicher Vertreter des Gemeinschuldners hinsichtlich der Insolvenzmasse ist, die „Organtheorie“, nach der der Verwalter Organ oder Repräsentant der Insolvenzmasse ist, der eine Rechtsfähigkeit oder Quasi-Rechtsfähigkeit zugesprochen wird, und die ganz vorherrschende und auch hier vertretenen „Amtstheorie“, wonach der Verwalter kraft des ihm übertragenen Amtes, materiellrechtlich wie prozessual im eigenen Namen und mit Wirkung für und gegen die Masse handelt. Einer näheren Eingehung auf diesen Streit bedarf es an dieser Stelle aber nicht. - Siehe ausführlich hierzu Uhlenbruck/Uhlenbruck, § 56 Rn.66ff. m.w.N. 
alleinigen Kenntnissen über die Geschäfte des Gemeinschuldners und damit über die Berechtigung der angemeldeten Insolvenzforderungen. ${ }^{222}$ Dem Insolvenzgericht ist eine solche Entscheidungs-/ Mitwirkungsbefugnis dagegen gänzlich versagt. Ihm obliegt neben der Terminleitung ${ }^{223}$ und der Hinweispflicht nach $\S 175$ II InsO bezüglich der angemeldeten Insolvenzforderungen nur eine rein beurkundende Tätigkeit, nämlich die Protokollierung des Verhaltens der Widerspruchsberechtigten (vgl. § 178 II 1, 2 InsO) $)^{224}$, nicht aber eine gerichtliche Entscheidung ${ }^{225}$ über den Bestand der Forderung oder der ihr entspringenden Berechtigung auf Feststellung und Teilnahme am Insolvenzverfahren. ${ }^{226}$ Vielmehr steht dies allenfalls dem im $\S 180$ I 2, 3 InsO bezeichneten Prozessgericht zu; dies aber ausdrücklich auch nur nach durchgeführtem Prüfungstermin und mangelnder Anerkennung der angemeldeten Forderung durch einen der Widerspruchsberechtigten (arg. §§ 180 I 1, 181 InsO) ${ }^{227}$, während dem Insolvenzgericht als solchem insoweit jegliche Kompetenz bewusst vorenthalten wurde. ${ }^{228}$

Zwar sieht das Gesetz vor, dass nur „Insolvenzforderungen“ angemeldet werden können (§ 174 I 1 InsO). Dies bedeutet aber nicht mehr, als dass das Insolvenzfeststellungsverfahren der $\S \S 174 \mathrm{ff}$. InsO - lediglich - für Forderungen eröffnet ist, die mit der Rechtsbehauptung angemeldet werden, Insolvenzforderungen zu sein und deshalb am Verfahren teilhaben dürfen. Die Feststellung, ob diese Behauptung zutrifft, ob also die tatsächlichen und rechtlichen Voraussetzungen des Bestehens der

222 Vgl. bereits KO-Motive, S.362 = Hahn, KO, S.325: „...der Verwalter [vereinigt] in seiner Person zugleich die Funktion des gemeinrechtlichen Kontradiktors...und [sei] in pflichtmäßiger Wahrnehmung der Interessen aller Gläubiger berufen...“; siehe auch Eckardt, ZIP 1993, 1765 (1766).

223 Siehe aber auch hinsichtlich zu entscheidender Anträge etwa bzgl. einer Stimmrechtsfeststellung gem. § 77 II 3 InsO unter D.VI.2.c) (S.105ff.).

224 Entspricht inhaltlich $\S 145$ I KO.

225 Das Insolvenzverfahren ist kein Erkenntnisverfahren. Siehe bereits unter D.I. (S.23ff.).

226 Vgl. KO-Motive, S.361 = Hahn, KO, S.324: „Weder [sei] ein Rechtsstreit ...vorhanden, noch soll[e]...der Richter eine Entscheidung über den Anspruch fällen“ und KO-Motive, S.363 = Hahn, KO, S.326: „...Feststellung des Ergebnisses [des Prüfungstermins] durch das Konkursgericht...“; ferner allg. Meinung: RGZ 37, 386 (388); Jaeger/Weber, KO, § 145 Rn.3; Gaul, in: FS/Weber, 155 (175); Uhlenbruck/Uhlenbruck, § 178 Rn.5; MüKo-InsO/Schumacher, § 178 Rn.47; Küber/Prütting/Pape, § 178 Rn.3; Häsemeyer, InsO, Rn.22.15; Bähr, InVo 1998, 205 (208); KS/Eckardt, S.761 Rn.35; Eckardt, ZIP 1993, 1765 (1766) m.w.N.

227 Entsprechen inhaltlich § 146 I 1, IV KO.

228 KO-Motive, S.365 = Hahn, KO, S.328: Schon die Zuweisung des Konkursverfahrens an den Einzelrichter mache es „ganz unthunlich“, die Spezialprozesse über bestrittene Forderungen vor das Konkursgericht zu ziehen. 
Forderung erfüllt sind und ob die Forderung als Insolvenzforderung zu qualifizieren ist, ${ }^{229}$ ist dagegen gerade in diesem Verfahren, nämlich im dafür vorgesehenen Prüfungstermin ( $\S 176$ InsO $)^{230}$, und nur von den Widerspruchsberechtigten zu treffen. Etwas anderes ist nicht mit der letztlich aus der Feststellung zur Tabelle erwachsenen gesetzlichen Rechtskraftwirkung für und gegen den Insolvenzverwalter und alle Insolvenzgläubiger (vgl. § 178 III InsO) ${ }^{231}$ vereinbar, die gerade an die getroffenen Entscheidungen der Widerspruchsberechtigten untereinander im Prüfungstermin anknüpft (vgl. § 178 I 1 InsO) und eben nicht (auch) an eine etwaige davon unabhängige Entscheidung des Verwalters oder des Insolvenzgerichts bereits im Vorfeld des (bloßen) Anmeldeverfahrens. Dies widerspräche zudem der ausdrücklich bekundeten Absicht des Gesetzgebers, durch die Ausgestaltung des Feststellungsverfahrens zu gewährleisten, dass die Befugnisse der einzelnen Gläubiger untereinander im Prüfungsverfahren alsbald und umfassend geklärt werden und danach gerade nicht mehr in Frage gestellt werden können. ${ }^{232}$ An dieser insolvenzverfahrensrechtlich stufenweise abgestimmten Klärungsmöglichkeit - Prüfungstermin, Widerspruchsmöglichkeit und Übergang ins prozessuale Feststellungsverfahren (vgl. § 179ff. InsO) - könnte der bereits im Anmeldeverfahren materiellrechtlich zurückgewiesene Insolvenzgläubiger aber erst gar nicht teilnehmen und wäre darüber hinaus von einer Durchsetzung seiner Interessen auch auf anderem Wege abgeschnitten (vgl. §§ 87, 89 InsO).

Hier also dem Insolvenzgericht ${ }^{233}$ oder auch dem die Anmeldungen entgegennehmendem Insolvenzverwalter ein unabhängiges und eigenständiges materielles Zurückweisungsrecht im Vorfeld des Prüfungstermins für die einzelnen Forderungsanmeldungen einzuräumen mit der Folge, dass die entsprechenden Forderungen zurückgewiesen und nicht in die Tabelle für ihre eigentliche Prüfung durch sämtliche

\footnotetext{
Siehe dazu auch C.I. (S.10ff.).

Entspricht inhaltlich dem $\S 141$ S.1 KO.

Entspricht inhaltlich den $\S \S 145 \mathrm{II}, 147 \mathrm{KO}$.

232 KO-Motive, S.357 = Hahn, KO, S.322: „Der Entwurf [sei]...bestrebt, einmal dem Verfahren eine [im Vergleich zur preußischen KO von 1855] noch größere Beschleunigung...zu geben, sodann dasselbe so zu konstruieren, daß dadurch sowohl für alle späteren Verhandlungen im Konkurse, als auch...über den Konkurs hinaus für die Verfolgung der Ausfälle gegen den gewesenen Gemeinschuldner eine judikatmäßig feste und leicht übersichtliche Grundlage gewonnen werde.“

233 Siehe auch unter D.IV.5.e) (S.67ff.).
} 
Widerspruchsberechtigten im dafür vorgesehenen Prüfungstermin aufgenommen würden, ist abzulehnen. ${ }^{234}$ Der Insolvenzverwalter macht sich zwar regelmäßig schon bei der Forderungsanmeldung Gedanken über die materielle Zulässigkeit der Insolvenzforderung und muss jede Forderung insoweit hinsichtlich eines (späteren) Widerspruchs prüfen, ${ }^{235}$ nur dürfen diese Überlegungen eben nicht mit in seine Entscheidung hinsichtlich ihrer Zulassung zum Insolvenzverfahren und ihrer Aufnahme in die Tabelle einfließen.

Für die Behandlung der unzulässigen Anmeldungen bedeutet dies somit, dass sämtliche Anmeldungen, die mit der Rechtsbehauptung angemeldet werden, Insolvenzforderungen zu sein, und dabei alle sonstigen formellen (Mindest-)Voraussetzungen der Anmeldung erfüllen, zum Verfahren zuzulassen, in die Tabelle aufzunehmen und zur Prüfung im Prüfungstermin zu stellen sind. ${ }^{236}$ Dies hat unabhängig davon zu erfolgen, dass die angemeldeten „Insolvenzforderungen“ wegen ihrer erkennbaren tatsächlichen Eigenschaft - etwa als Masseforderung oder nicht zugelassene nachrangige Insolvenzforderung - als unzulässig anzusehen und damit nicht für das Anmelde- und Feststellungsverfahren der $\S \S 174 \mathrm{ff}$. InsO bestimmt sind. ${ }^{237}$

Daher bleibt festzuhalten, dass eine materielle Zurückweisungskompetenz im Vorfeld des Prüfungstermins somit nicht besteht. Zuständig für den letztendlichen Ausschluss materiellrechtlich unzulässiger Forderungen von der weiteren Teilnahme am Verfahren und somit auch von der Teilhabe an der Insolvenzmasse ist damit einzig - durch Erhebung des noch zu behandelnden Widerspruchs ${ }^{238}$ - die Gemeinschaft bzw. jeder Einzelne der Widerspruchsberechtigten im Prüfungstermin.

\footnotetext{
234 Insofern ist Uhlenbruck/Uhlenbruck, § $175 \mathrm{Rn} .3 \mathrm{zu}$ widersprechen, der dem Insolvenzverwalter diese Zurückweisungsbefugnis bei „evidenten inhaltlichen [=materiellen] Mängeln“ der Anmeldung zusprechen will.

235 Dazu unter E.I.4.a) (S.141ff.).

236 So auch MüKo-InsO/Nowak, § 174 Rn.29; FK/Kießner, § 174 Rn.36; GrafSchlicker/Graf-Schlicker, § 175 Rn.5; Kilger/K.Schmidt, KO, § 140 Rn.2; KS/Eckardt, S.751 Rn.15; Eckardt, ZIP 1993, 1765 (1767).

238 Siehe hierzu unter E.I.4. (S.140ff.).
} 
Insofern kommen für eine Zurückweisung im Rahmen einer Vorprüfung nur diejenigen Forderungen in Frage, denen es (1) an der besagten Rechtsbehauptung „Insolvenzforderung zu sein“ in der Anmeldung fehlt - was in der Praxis der eher unwahrscheinlichste Fall wäre ${ }^{239}$ - oder die (2) aus rein formeller Sicht unzulässig sind, weil ihnen für eine zulässige Anmeldung die nötigen Voraussetzungen hinsichtlich des Inhalts und der Form fehlen.

Dass diese Fälle schon aus verfahrensrechtlicher Sicht keine Aufnahme in die Tabelle finden können, ist allgemein unbestritten. Nur wem diese wichtige Prüfungs- und Zurückweisungskompetenz im Rahmen einer Vorprüfung im Anmeldeverfahren nun genau zusteht, darüber gehen die Meinungen auseinander und soll im Folgenden nicht nur aufgrund der Erörterung des insolvenzrechtlichen Verfahrensablaufs, sondern auch im Hinblick auf die Folgen einer Abweisung oder Zulassung zum Insolvenzverfahren geklärt werden.

Dabei soll zunächst ein kurzer Rückblick auf die Problematik innerhalb der Konkursordnung genommen werden, um Rückschlüsse aus der Gesetzesund Meinungsentwicklung für die jetzige Insolvenzordnung ziehen zu können.

\section{c) Prüfung durch Urkundsbeamte unter der Konkursordnung}

Bis in die letzte Geltungsphase der Konkursordnung war umstritten, ob die Kompetenz, formell unzulässige Forderungsanmeldungen zurückzuweisen, ausschließlich dem Konkursgericht im Rahmen seiner (Vor-) Prüfungspflicht - ob die angemeldete Forderung nach § 141 I KO zur Erörterung im Prüfungstermin zuzulassen ist - zusteht, oder ob sie daneben auch bzw. bereits dem Urkundsbeamten der Geschäftsstellen im Anmeldestadium zusteht, der jede Forderung nach der Anmeldung auf der Geschäftsstelle gem. § 140 II KO sofort in die Tabelle einzutragen hat.

239 Z.B. wenn ausdrücklich eine Masseforderung oder eine nicht zur Anmeldung gem. $\S 174$ III InsO zugelassene nachrangige Insolvenzforderung mit dem Hinweis auf ihren Nachrang angemeldet wird. 
Diese Fragestellung ist von Bedeutung, da eine ähnliche Konstellation heute zwischen dem Insolvenzverwalter und dem Insolvenzgericht besteht. Zunächst ist festzuhalten, dass unter Konkursgericht - wie auch heute unter Insolvenzgericht - in erster Linie der zuständige Rechtspfleger zu verstehen ist, dem seit Inkrafttreten der Konkursordnung im Jahre 1879 nach und nach im Rahmen des Rechtspflegergesetzes (RpflG) von $1957^{240}$ und $1969^{241}$ der Großteil der Befugnisse des eigentlichen Konkursrichters übertragen worden ist. Dadurch oblag schon unter der Konkursordnung gem. § 3 Nr.2 e) RpflG ${ }^{242}$ die generelle Zuständigkeit für das Konkursverfahren zuletzt dem Rechtspfleger, soweit nicht einzelne Geschäfte dem Richter vorbehalten waren. ${ }^{243}$ Desgleichen wurde der Aufgabenbereich des Urkundsbeamten der Geschäftsstelle nach § 140 II KO schon damals vom RpflG nicht berührt (vgl. § 26 RpflG) ${ }^{244}$ und stellte eine selbstständige und unabhängige Tätigkeit dar. ${ }^{245}$ Die Geschäftsstelle nimmt bei Gerichten und der Staatsanwaltschaft alle gerichtsentlastenden Aufgaben wahr, ${ }^{246}$ die nicht ausdrücklich dem Richter, Staatsanwalt, Rechtspfleger oder Amtsanwalt zugewiesen sind. Insbesondere nehmen die Urkundsbeamten der Geschäftsstelle Beurkundungen vor, erteilen Ausfertigungen gerichtlicher Entscheidungen, einfache Vollstreckungsklauseln (für qualifizierte ist der Rechtspfleger zuständig, vgl. § 20 Nr.12 RpflG) oder fungieren als Protokollführer. Die durch das RpflG zur Entlastung vorgenommene Aufgabenübertragung vom Konkursrichter auf den Rechtspfleger bei gleichzeitigem Nebeneinander von Rechtspfleger und Urkundsbeamtem der Geschäftsstelle war schließlich der stete Anlass der

40 BGBl. I vom 8.2.1957, S.18, 44.

241 BGBl. I vom 5.11.1969, S.2065.

$242 \S 3$ Nr.2 e) RpflG hat sich unter der Insolvenzordnung bis auf die Wortaustausch „Konkurs“ - „Insolvenz“ nicht geändert.

243 Die Ausnahmeregelungen der $\S \S 18,19$, 19a RpflG unter der Konkursordnung und deren inhaltliche ähnlichen Nachfolgeregelungen unter der jetzigen Insolvenzordnung, sind für die hier zu untersuchenden Problematik nicht weiter von Bedeutung.

244 Entspricht inhaltlich weitestgehend dem § 25 RpflG (1957).

245 Zur Konkurs- wie auch heutiger Insolvenzordnung siehe Bassenge/Herbst, RpflG, $\S$ 26; Jaeger/Weber, KO, § 140 Anm.2a; Kilger/K.Schmidt, KO, § 140 Anm.2; Bassenge/Herbst/Roth, RpflG, Einleitung Rn.8; Arnold/Herrmann, RpflG, § 26 Rn.3.; Kuhn/Uhlenbruck, KO, § 140 Rn.3.

246 Die Geschäftsstelle geht auf die mit dem Gerichtsverfassungsgesetz eingeführte Gerichtsschreiberei zurück (§ 154 GVG a. F.). Ziel dieser Maßnahme war es, die Richterschaft zu entlasten und Gerichtstätigkeiten, zu deren Wahrnehmung es keiner akademischen Ausbildung bedurfte, auf Beamte des mittleren oder gehobenen Justizdienstes zu übertragen. 
Diskussion für oder gegen ein eigenständiges formelles Vorprüfungs- und Zurückweisungsrecht auch des Urkundsbeamten. ${ }^{247}$ Zumindest insoweit, als der Urkundsbeamte nicht zugleich auch Rechtspfleger war (vgl. § 27 I RpflG), da ihm als Rechtspfleger die entsprechende Prüfungs- und Zurückweisungsbefugnis insoweit zustand.

War zurückblickend in den Anfängen der Konkursordnung die vorgenommene Tabelleneintragung der schriftlich oder zu Protokoll gegebenen Anmeldungen durch den damaligen Gerichtsschreiber wohl nur eine rein beurkundende Tätigkeit ${ }^{248}$ und wurde im Laufe der Zeit durch § 21 I Nr.5 RpflG (1957) ${ }^{249}$ zunächst die Vorprüfung der Anmeldungen ${ }^{250}$ und durch $\S 3$ Nr.2 e) RpflG (1969) schließlich die gesamte formelle Zulassungsprüfung im Rahmen des $\S 141 \mathrm{KO}$ auf den Rechtspfleger übertragen, so fand eine Regelung - mit Ausnahme des § 26 RpflG - für die Abgrenzung der Zuständigkeit des Rechtspflegers im Verhältnis zum Urkundsbeamten jedoch nicht weiter statt. Eine dem früheren § 21 I Nr.5 RpflG (1957) entsprechende Regelung fehlte ab 1969. Trotz bzw. gerade wegen dieses Fehlens sprach sich die herrschende Meinung ${ }^{251}$ in der letzten Geltungsphase der Konkursordnung für eine Art „entlastende Parallelverschiebung der Zuständigkeiten und Aufgaben“ aus, so dass dem Urkundsbeamten der Geschäftsstelle nicht nur ein - soweit ersichtlich unstreitiges - formelles Vorprüfungsrecht hinsichtlich der gesetzlichen Erfordernisse einer wirksame Anmeldung gem. § 139 S.1

247 Zum fast 100-jährigen Verlauf des Streitstandes siehe Uhlenbruck, Rpfleger 1991, $445 f f$.

248 Vergleiche hierzu die KO-Motive, S.360f. = Hahn, KO, S.324 wo es heißt: „Sämtliche Anmeldungen...sollen von dem Gerichtsschreiber...sofort nach ihrem Eingange in eine Tabelle eingetragen werden, welche als urkundliche Basis [diene]...", in Verbindung mit dem Protokoll der 13. Sitzung der Kommission, S.91: „Der Inhalt...der Anmeldung [und damit der vorgenommene Tabelleneintrag] sei nicht bindend, der Betreffende könne denselben später modifizieren, auch ganz von ihm abgehen." Siehe auch Jaeger, KO, § 140 Rn.2; Wolff, KO, § 140 Rn.2; Uhlenbruck, Rpfleger 1991, 445 (446) m.w.N.

249 § 21 I Nr.5 RpflG (1957): „Folgende Geschäfte im Verfahren der Konkursordnung werden dem Rechtspfleger übertragen: ... 5. der Erlaß von Verfügungen, durch die Auflagen zur Ergänzung oder Richtigstellung der Anmeldungen (§§ 139, 140 der Konkursordnung) gemacht werden; ... “; Bund Deutscher Rechtspfleger, Rechtspflegergesetz, S.24.

250 Siehe auch Arndt, RpflG 1957, § 21 Rn.19; Hofmann/Kersting, RpflG 1957, § 21 Ziff.5, S.254.

251 Kuhn/Uhlenbruck, § 140 Rn.3; Gottwald/Eickmann, KO, $\S 63$ Rn.16; Kilger/K.Schmidt, KO, § 140 Anm.2; Eickmann, Rpfleger 1970, 318 (318f.); Kübler, in: FS/Henckel, 495 (498f.); Uhlenbruck, Rpfleger 1991, 445 (446f.). 
$\mathrm{KO}^{252}$ zugesprochen wurde, sondern darüber hinaus, im Rahmen seiner Zuständigkeit für die Tabellenführung auch ein eigenes Zurückweisungsrecht; unabhängig vom zuständigen Rechtspfleger. ${ }^{253}$

Aufgrund seines Hinauswachsens aus der rein beurkundenden Funktion eines Gerichtsschreibers alter Prägung und insbesondere mit Hinweis auf den Sinn und Zweck des § 140 KO und der Stellung des Urkundsbeamten als selbstständiges Rechtspflegeorgan unterlag es seiner Amtspflicht, die formelle Ordnungsmäßigkeit der Anmeldung zu prüfen, gegebenenfalls die erforderlichen Ergänzungen und Klärungen anzuregen ${ }^{254}$ und nur für den Fall, dass diese unterblieben, die Aufnahme in die Tabelle zu verweigern. Auch wenn dem Urkundsbeamten hier scheinbar nur eine Entgegennahme und Beurkundung von Erklärungen oblag, so kann diese Verpflichtung doch vernünftigerweise nicht auf Erklärungen jeglicher Art, mit denen irgendein Begehren an das Insolvenzgericht gerichtet ist, bezogen werden. Denn die Tabelle ist vielmehr nur zur Aufnahme von ordnungsgemäßen Begehren auf Befriedigung aus der Masse bestimmt und geeignet. So musste es richtigerweise bereits dem Urkundsbeamten obliegen, die Tabelle von anderen Erklärungen grundsätzlich freizuhalten, die sie unübersichtlich und in ihrer praktischen Brauchbarkeit beeinträchtigen würden. Hätte dem Urkundsbeamten diese Aufgabe nicht oblegen, wären für jedermann ohne Einhaltung der formellen Voraussetzungen die Anmeldung und die daraus resultierende Teilnahme am weiteren Konkursverfahren ermöglicht worden. Zudem wäre die Klärung von offensichtlichen formellen Unstimmigkeiten in das nachfolgende Prüfungsverfahren verschoben worden, in dem jedoch nach dessen Sinn und Zweck die Anmeldungen bzw. die angemeldeten Forderungen bereits für die Verfahrensbeteiligten prüfbar - eben mittels der formell

252 Entspricht dem $\S 174$ InsO. Zu den bereits erörterten Erfordernissen siehe nur zusammenfassend Fn.204, 205 (S.54).

253 Die Gegenmeinung sprach dem Urkundsbeamten nicht das Recht zu, die Eintragung der zur Konkurstabelle angemeldeten Forderungen verweigern zu dürfen. Dies teilweise selbst dann nicht, wenn die angemeldete Forderung offenkundig unwirksam ist, weil es am wesentlichen Inhalt einer Forderungsanmeldung fehlt oder wenn trotz Hinweises durch den Urkundsbeamten oder gar des Gerichts/Rechtspfleger, eine Ergänzung bzw. Berichtigung der Anmeldung nicht vorgenommen wurde. Die Eintragung sollte nur verweigert werden dürfen, wenn die Anmeldung durch Beschluss des Gerichts/Rechtspflegers zurückgewiesen worden ist. - Hess/Hess, KO, $\S 141$ Rn.4, 5; Jaeger/Weber, KO, § 140 Anm.2a; KS/Eckardt, S.752 Rn.18; Häsemeyer, KO, S.515.

254 Siehe zu diesem wünschenswerte Hinweis bereits durch den damaligen Gerichtsschreiber bei Jaeger, KO 1904, § 140 Rn.2; Wolff, KO, § 140 Rn.2. 
ordnungsgemäßen Anmeldung - vorliegen sollten. Zudem hätte bei einer Zurückweisung der formell unzulässigen Anmeldung erst im Prüfungstermin für den Anmeldenden die Gefahr bestanden, seine Forderung aufgrund der mittlerweile verstrichenen Anmeldefrist des $\S 14$ I $1 \mathrm{GesO}^{255}$ u.U. nicht mehr geltend machen zu können. Weiterhin wäre nicht nur die angestrebte Entlastung des Gerichts durch den Urkundsbeamten erfolglos geblieben, sondern auch das gesamte Verfahren unnötig in die Länge gezogen worden.

Letztlich war eine Zurückweisung bereits durch den Urkundsbeamten hinsichtlich wesentlicher Mängel im Rahmen des § 139 S.1 KO sowohl verfahrenstechnisch als auch für den Anmeldenden unschädlich, da auch der Rechtspfleger durch seine von Amts wegen (§ 72 KO i.V.m. § 139 ZPO) auferlegte (Vor-) Prüfungspflicht im Rahmen des § 141 I KO gehalten war, mangelhafte Anmeldungen zurückzuweisen und im Prüfungstermin nicht zur Erörterung zu stellen. ${ }^{256}$

Letztlich stand dem Anmeldenden gegen die Entscheidung des Urkundsbeamten immer noch die Möglichkeit der Erinnerung gem. §§ 72, 73 II KO, § $576 \mathrm{I} \mathrm{ZPO}^{257}$ zur Verfügung, über die nicht der Rechtspfleger, sondern sogar der Konkursrichter zu entscheiden hatte (§ 4 II Nr.3 RpflG). ${ }^{258}$

\section{d) Prüfung durch Insolvenzverwalter unter der Insolvenzordnung}

Die Zuständigkeit für die Annahme der Insolvenzforderungsanmeldungen während des gesamten Insolvenzverfahrens ist durch die Insolvenzordnung gem. § 174 InsO vollständig auf den Insolvenzverwalter übergegangen. Insofern ist fraglich, inwieweit die dargelegten Kompetenzen unter der Konkursordnung auf den Insolvenzverwalter und das Verfahren unter der Insolvenzordnung übertragen werden können bzw. welche Abweichungen,

Siehe dazu auch unter D.IV.7. (S.72ff.).

Allgemeine Meinung siehe nur Kuhn/Uhlenbruck, KO, § 141 Rn.2a m.w.N.

Baumbach/Lauterbach/Albers/Hartmann, ZPO (1998), § 576 Rn.3; heute in $\S 573$ ZPO geregelt.

258 Kuhn/Uhlenbruck, KO, § 141 Rn.2a; Gottwald/Eickmann, KO, § 63 Rn.16; Einzelheiten bei Uhlenbruck, Rpfleger 1991, 445ff. 
insbesondere hinsichtlich möglicher Rechtsbehelfe, gegen eine Zurückweisung bestehen.

Durch diese umfassende Kompetenzverlagerung auf den Insolvenzverwalter und die damit vom Gesetzgeber ausdrücklich beabsichtigte (weiteren) Entlastung der Insolvenzgerichte ${ }^{259}$ entspricht es nur dem Sinn dieser Verlagerung, auch die zuvor dargelegte formelle Vorprüfungspflicht des Urkundsbeamten der Geschäftsstelle bzw. des Rechtspflegers und dessen Zurückweisungsbefugnis im Anmeldeverfahren ${ }^{260}$ vollständig auf den Insolvenzverwalter zu übertragen. ${ }^{261}$ Auch wenn der Insolvenzverwalter gleichzeitig Verfahrens-beteiligter ist und damit in die Nähe der Stellung einer Partei rückt, ${ }^{262}$ übt der Insolvenzverwalter durch seine ihm gerichtlich übertragene Amtsstellung dennoch die bisherige Funktion des Urkundsbeamten/Rechtspflegers aus und kann somit die Eintragung der unzulässigen oder formell unvollständigen Forderungsanmeldungen verweigern. Zudem widerspräche eine zusätzliche bzw. erneute vollständige gerichtliche Vorprüfung der Anmeldungen durch das Insolvenzgericht nach Niederlegung der Tabelle $e^{263}$ der Intention des Gesetzgebers, die Insolvenzgerichte $\mathrm{zu}$ entlasten, und findet im eigentlichen Anmeldeverfahren unter der Insolvenzordnung somit grundsätzlich nicht mehr statt. Ein formelles Vorprüfungsrecht des Insolvenzverwalters ist dabei nicht nur zwangsläufig Folge seiner Pflicht zur ordnungsgemäßen Tabellenführung, wie sie schon dem Urkundsbeamten oblag, sondern zugleich auch prozessökonomisch sinnvoll; denn durch die Vorprüfung wird der Insolvenzverwalter nicht gezwungen, jede unzulässige Anmeldung einzutragen, um später im Prüfungstermin die Mängel zu beanstanden und die Forderung $\mathrm{zu}$ bestreiten. Wie schon der

259 Begr. des Rechtsausschuss zu § 32 (§ 28 InsO) RegE, BT-Drucks. 12/7302, S.159.

260 Zumal § 175 InsO inhaltlich dem § 140 KO entspricht. Vgl. auch Begr. zu § 202 (§ 175 InsO) RegE, BT-Drucks. 12/2443, S.184.

261 HK/Irschlinger, § 175 Rn.4, 7; KS/Wellensiek, S.405 Rn.10; Graf-Schlicker/GrafSchlicker, § 174 Rn.22; BK/Breutigam, § 175 Rn.6; Kübler/Prütting/Bork/Pape, $\S 174$ Rn.21f. und $\S 175$ Rn.2f.; Gottwald/Eickmann, InsO, § 63 Rn.15; Uhlenbruck/Uhlenbruck, § 174 Rn.3; Braun/Specovius, § 175 Rn.6; wohl auch Nerlich/Römermann/Becker, § 175 Rn.4.

So auch schon zur GesO: Kübler, in: FS/Henckel, 495 (499f.); Haarmeyer/Wutzke/Förster, GesO, § 11 Rn.56f.

262 KS/Eckardt, S.753 Rn.18.

263 So etwa MüKo-InsO/Nowak, § 174 Rn.31; Merkle, Rpfleger 2001, 157 (163ff.); Bähr, InVo 1998, 205 (208); für eine ausschließlich Vorprüfungspflicht des Insolvenzgerichts KS/Eckhardt, S.743 Rn.18, 23; Hess, InsO, § 175 Rn.19. 
Urkundsbeamte kann nämlich auch der Insolvenzverwalter nicht verpflichtet sein, jede Anmeldung in die Tabelle einzutragen. Notwendige Konsequenz der Vorprüfungspflicht des Insolvenzverwalters ist zugleich auch die Berechtigung, unzulässige oder formell unvollständige Anmeldungen zurückzuweisen, da die Prüfung der Insolvenzforderung im weiteren Verlauf des Verfahrens nicht möglich wäre. ${ }^{264}$ Einer Zurückweisung ist jedoch eine Hinweis- und Beanstandungspflicht (§ 139 ZPO i.V.m. \& 4 InsO) des Insolvenzverwalters über offensichtliche formelle Anmeldungsmängel gegenüber dem Anmeldenden vorgeschaltet, ${ }^{265}$ um dem Anmeldenden die Möglichkeit zu geben, die beanstandeten Mängel zu beheben. ${ }^{266}$ Wird der Mangel nicht behoben oder wird die unzulässige Anmeldung nicht zurückgenommen, so ist der Insolvenzverwalter nicht nur berechtigt, sondern vielmehr verpflichtet, die Anmeldung zurückzuweisen. Daneben ist der Insolvenzverwalter auch gehalten, den Anmeldenden auf sonstige offensichtliche Mängel und Unklarheiten (z.B. fehlende Unterlagen, Ergänzungen) im Vorfeld des Prüfungstermins hinzuweisen, die zu einem Bestreiten der angemeldeten Insolvenzforderung führen könnten. Dies dient durchaus der Beschleunigung des Verfahrens, jedoch kann in diesen Fällen - im Gegensatz zu den offensichtlichen formellen Mängeln i.S.d. $\S 174$ InsO, die schon einer Aufnahme in die Tabelle entgegen stehen keine Hinweispflicht des Insolvenzverwalters bestehen, da der Anmeldende das Risiko seiner unzulänglichen Forderungsanmeldung grundsätzlich selbst zu tragen hat. ${ }^{267}$

\section{e) Rechtsbehelfe gegen die Zurückweisung des Insolvenzverwalters}

Die Zurückweisung der formell unzulässigen Anmeldung durch den Insolvenzverwalter ist keine gerichtliche Entscheidung und erfolgt daher

\footnotetext{
Siehe bereits unter D.IV.5. (S.53ff.).

Vgl. im Ergebnis OLG Stuttgart ZinsO 2008, 627 (628), das jedoch (ohne weitere Begründung) der Ansicht ist, dass sich diese Pflicht des Insolvenzverwalters nicht aus $\S 4$ InsO i.V.m. § 139 ZPO ergibt. Dem ist jedoch aufgrund des dargelegten Eintretens des Insolvenzverwalters in den vormaligen Aufgabenbereich des Urkundsbeamten/Rechtspflegers nicht zuzustimmen.

267 Im Ergebnis auch OLG Stuttgart ZinsO 2008, 627 (628). Zur verfahrensrechtlichen Last seitens des Anmeldenden siehe bereits unter D.II. (S.30ff.).
} 
nicht in Form eines Beschlusses. Die Entscheidung des Insolvenzverwalters entspricht inhaltlich und in der Folge ${ }^{268}$ zwar der des Urkundsbeamten, sie kann aber aufgrund des eigenständigen und unabhängigen Amtes des Insolvenzverwalters nicht mit dieser gleichgesetzt werden, so dass etwa die Erinnerung (§ 573 I ZPO) - anders als unter der Konkursordnung gegen die Entscheidung des Urkundsbeamten - gegen die Entscheidung des Insolvenzverwalters nicht möglich ist. Der Insolvenzverwalter handelt in seinem eigenen Aufgabenund Pflichtenkreis. ${ }^{269}$ Daher handelt es sich bei der Zurückweisung vielmehr um eine („einfache“) Prozesshandlung im Rahmen seines Amtes, die auch nicht an eine bestimmte Form gebunden ist. Gleichwohl sollte die Zurückweisung schriftlich und mit Begründung erfolgen. Dies hat zur Folge, dass dem Anmeldenden gegen die Zurückweisung der Anmeldung aus formellen Mängeln durch den Insolvenzverwalter auch keine förmlichen Rechtsbehelfe zur Verfügung stehen. Fraglich ist daher, welche Möglichkeiten für den Anmeldenden bestehen, gegen die Entscheidung des Insolvenzverwalters vorzugehen.

\section{aa) Einschreiten des Insolvenzgerichts im Aufsichtswege}

Der Anmeldende kann einerseits ein Einschreiten des Insolvenzgerichts im Wege der Aufsicht gem. § 58 II 1 InsO anregen. ${ }^{270}$ Das Insolvenzgericht kann den Insolvenzverwalter dann nach eigener formeller Prüfung der Anmeldung im Rahmen des allgemeinen Anmeldeverfahrens anweisen, die angemeldete Forderung im Verfahren zu berücksichtigen und in die Tabelle einzutragen. Ist der Insolvenzverwalter mit der Anweisung des Insolvenzgerichts nicht einverstanden, so kann er dagegen - nach vorheriger gerichtlicher Androhung von Zwangsgeld wegen der Nichtaufnahme der Forderung - gem. § 58 II 3 InsO mit der sofortigen

268 Folge: Keine Berücksichtigung bzw. Aufnahme der formell unzulässigen Anmeldung bzw. der in ihr enthaltenen Forderung(en) ins Insolvenzverfahren.

269 Siehe auch schon unter D.IV.2.b) (S.38ff.).

270 Gem. § 5 I InsO wird das Insolvenzverfahren vom Amtsbetrieb beherrscht, so dass gegenüber dem Insolvenzgericht kein Rechtsanspruch auf aufsichtsrechtliches Einschreiten besteht. - Kübler/Prütting/Bork/Pape, § 175 Rn.4; MüKo-InsO/Graeber, $\S 58$ Rn. 12, 59; Uhlenbruck/Uhlenbruck, § 175 Rn.6; Graf-Schlicker/Graf-Schlicker, § 175 Rn.7; HK/Irschlinger, § 175 Rn.8; Braun/Specovius, § 175 Rn.10. 
Beschwerde vorgehen. Neben der Möglichkeit der Verhängung eines Zwangsgeldes nach § 58 II InsO besteht sogar die Möglichkeit der Entlassung des Insolvenzverwalters aus wichtigem Grund nach § 59 InsO. Zudem haftet der Insolvenzverwalter persönlich gem. § 60 InsO, wenn er pflichtwidrig eine Forderung nicht in die Tabelle aufnimmt, obwohl ein Anmeldungsmangel tatsächlich nicht vorgelegen hat. ${ }^{271}$ Soweit wird es der Insolvenzverwalter in der Praxis jedoch nicht kommen lassen, sondern die angemeldete „Forderung“ in die Tabelle aufnehmen, sie dann aber bestreiten.

\section{bb) Prüfung durch das Insolvenzgericht im Prüfungstermin}

Dennoch bleibt fraglich, wie der Anmeldende die Zulassung und Aufnahme seiner Anmeldung bzw. Insolvenzforderung in die Tabelle bewirken kann, falls sich der Insolvenzverwalter trotz Zwangsgeldauferlegung weigert, die Anmeldung anzunehmen und die Forderung in die Tabelle aufzunehmen. In diesem Fall wird zu überlegen sein, inwiefern die Entscheidung des Insolvenzverwalters nicht gleichwohl einer weiteren bzw. zusätzlichen Prüfung durch das Insolvenzgericht zugänglich sein kann bzw. muss. Dies wird zwar nicht im Rahmen des allgemeinen Anmeldeverfahrens, da die formelle Prüfung und Tabellenführung insoweit vollständig auf den Insolvenzverwalter übergegangen ist, wohl aber im Rahmen des Prüfungs-/Schlusstermins ${ }^{272}$ möglich sein. Denn auch das Insolvenzgericht hat, wenn durch Beanstandungen des Insolvenzverwalters hierzu Anlass besteht, von Amts wegen (§ 139 ZPO i.V.m. § 4 InsO) die formelle Zulässigkeit der entsprechenden Anmeldung zu prüfen. ${ }^{273}$ Das bedeutet jedoch nicht, dass das Insolvenzgericht sämtliche Anmeldungen auf ihre Zulässigkeit zu prüfen hat, sondern dass sich die Prüfung nur auf die Fälle beschränken wird, in denen der Insolvenzverwalter die Forderungen des anmeldenden Insolvenzgläubigers beanstandet oder

Kübler/Prütting/Bork/Pape, $\S 175$ Rn.4; Braun/Specovius, $\S 175$ Rn.10; Uhlenbruck/Uhlenbruck, § 175 Rn.6 m.w.N.

Siehe auch unter E.II.3.a) (S.192ff.).

273 HK/Irschlinger, § 176 Rn.3; KS/Eckardt, S.755 Rn.23; Uhlenbruck/Uhlenbruck, $\S 176$ Rn.2; BK/Breutigam, § 176 Rn.1; Hess, InsO, § 176 Rn.5; MüKo-InsO/Nowak, $\S 176 \mathrm{Rn} .18$. 
bereits als unzulässig zurückgewiesen hat. Aufgrund dessen, dass es sich bei der Zurückweisung durch den Insolvenzverwalter um eine Prozesshandlung im Rahmen seines Amtes und nicht um einen gerichtlichen (rechtskräftigen) Beschluss handelt, ist es dem Anmeldenden in diesen Fällen möglich, die Zulässigkeit seiner (ggf. erneuten, vgl. § 177 InsO) Forderungsanmeldung im Prüfungstermin auf Antrag dann auch im Rahmen einer gerichtlichen Entscheidung prüfen $\mathrm{zu}$ lassen. ${ }^{274}$ Die Anmeldung und ihre formelle Prüfung erfolgt zwar (zunächst) beim im Prüfungstermin anwesenden Insolvenzverwalter (§ 174 I 1 InsO), ${ }^{275}$ kann aber bei Unstimmigkeiten über ihre formelle Zulässigkeit darüber hinaus unproblematisch auch von dem den Prüfungstermin leitenden Insolvenzgericht/Rechtspfleger geprüft werden. Mit der Niederlegung der Tabelle vor dem Prüfungstermin ( $\S 175$ I 2 InsO) geht auch die Tabellenführung auf das Insolvenzgericht über, ${ }^{276}$ so dass das Insolvenzgericht - im Gegensatz zum tabellenführenden Insolvenzverwalter während des allgemeinen Anmeldeverfahrens vor Niederlegung - die Eintragung der Insolvenzforderung in die Tabelle nunmehr selbst vornehmen und damit zur weiteren (materiellrechtlichen) Prüfung im Verfahren stellen kann. Die materiellrechtliche Prüfung obliegt jedoch wie bereits erörtert ${ }^{277}$ - allein dem Insolvenzverwalter und den Insolvenzgläubigern.

Diese beiden, an die Initiative des zurückgewiesenen Anmeldenden zu knüpfenden Antragsmöglichkeiten hinsichtlich einer gerichtlichen Überprüfung der Zulassungsentscheidung des Insolvenzverwalters im Rahmen eines Tätigwerdens im Aufsichtswege oder der Prüfung im Prüfungstermin, sind als eine Art Rechtsbehelf hinsichtlich der formellen Anmeldung anzusehen. Wie bereits aufgezeigt, ${ }^{278}$ stellt die Anmeldung des Insolvenzgläubigers zum Insolvenzverfahren die einzige Möglichkeit dar, einen eventuellen Anspruch mit all den damit verbundenen

\footnotetext{
274 Die erneute Anmeldung unterfällt dann wegen der bereits abgelaufenen Anmeldefrist den Voraussetzungen des § 177 InsO. - Uhlenbruck/Uhlenbruck, § 176 Rn.2; Kehe/Meyer/Schmerbach, ZInsO 2002, 615 (618).

275 Siehe dazu unter E.I.2.c) (S.136f.).

276 Siehe ausführlich unter D.V.2. (S.85ff.).

277 Siehe unter D.IV.5.b) (S.56ff.).

278 Siehe unter D.IV.5.a) (S.53ff.).
} 
Teilnahmerechten überhaupt überprüfen, feststellen oder gegebenenfalls ins ausdrücklich an das Anmelde- und Feststellungsverfahren anknüpfende gerichtliche Feststellungsverfahren (vgl. §§ 179ff. InsO) übergehen zu lassen. Daher kann aufgrund der Wichtigkeit des Zugangs zum Verfahren die Entscheidung hierüber nicht allein auf der Entscheidung eines Einzelnen - in diesem Fall des Insolvenzverwalters - beruhen, ohne dass diese einer anderweitig rechtlichen Würdigung zugänglich wäre. Eine formelle Prüfung der Anmeldungen durch das Insolvenzgericht findet daher auch nicht generell - da insoweit vollständig auf den Insolvenzverwalter übergegangen -, sondern nur in den beiden genannten Fällen auf Antrag des Betroffenen statt. ${ }^{279}$ Dadurch kommt es zu keinen (Prüfungs-)Überschneidungen und die klare Aufgaben- und Kompetenzverteilung bleibt gewahrt.

Sollte der Betroffene einen dieser Anträge stellen, ist jedoch vor einer gerichtlichen Entscheidung der Insolvenzverwalter wegen seiner umfassenden Kenntnis sämtlicher Umstände hinsichtlich seiner Beanstandung oder Zurückweisung der betroffenen Forderungsanmeldung anzuhören und dem Anmeldenden auch hier noch die Möglichkeit zu geben, eventuelle Mängel der Anmeldung zu beseitigen. ${ }^{280}$ Die endgültige Entscheidung auf Zulassung zum Insolvenzverfahren oder über die Zurückweisung der Anmeldung ergeht dann durch förmlichen Beschluss des Insolvenzgerichts, wobei auch eine einstweilige Zulassung zur Prüfung gem. §§ 56 II, 89 I ZPO i.V.m. § 4 InsO bei Gefahr in Verzug (z.B. wegen Ablaufs der Verjährungsfrist) möglich ist. Eine Feststellung kann dann jedoch nicht vor Beseitigung des Mangels erfolgen. Hat der Rechtspfleger entschieden, steht dem Anmeldenden hiergegen die sofortige Erinnerung, jedoch kein weiteres Rechtsmittel gegen die Entscheidung des zuständigen Richters zu (§ 11 II RPflG i.V.m. § 6 I InsO). ${ }^{281}$

279 Aufgrund der ohnehin relativ geringen formellen Anforderungen als Hürde für eine Anmeldung, sind diese beiden Anträge als Rechtsbehelfe für eine in seltenen Fällen tatsächlich mal aus formellen Gründen zurückgewiesene Anmeldung auch als ausreichend für eine Klärung durch das Insolvenzgericht anzusehen. MüKo-InsO/Nowak, § 176 Rn.23; Hess, InsO, § 176 Rn.7; Uhlenbruck/Uhlenbruck $\S 176$ Rn.2 m.w.N.

281 Siehe nur Uhlenbruck/Uhlenbruck, § 6 Rn.17 m.w.N. 


\section{Zwischenergebnis}

Somit bleibt bezüglich der anfangs aufgeworfenen Frage, ob und von wem in welchem Umfang formell unzulässige Anmeldungen geprüft und zurückgewiesen werden können bzw. müssen, festzuhalten, dass ausschließlich der Insolvenzverwalter eine Vorprüfungs- und Zurückweisungsbefugnis hinsichtlich offensichtlicher formeller Mängel der Insolvenzforderungsanmeldung im Rahmen des Anmeldeverfahrens hat. Dadurch nimmt der Insolvenzverwalter die zentrale Stellung im Rahmen des Anmeldeverfahrens ein. Aufgrund der Wichtigkeit des Zugangs zum Verfahren muss die Entscheidung des Insolvenzverwalters, eine Anmeldung aus formellen Mängeln zurückzuweisen, jedoch einer weiteren Überprüfung durch das Insolvenzgericht zugänglich sein. Eine rechtsbehelfsmäßige Überprüfung durch das Insolvenzgericht erfolgt auf Antrag des Zurückgewiesenen in zwei Fällen: zum einen im Rahmen der Anregung eines Einschreitens im Aufsichtwege gem. § 58 InsO und/oder zum anderen, im Rahmen der erneuten Anmeldung im Prüfungstermin. Darüber hinaus findet, aufgrund der Kompetenzübertragung auf den Insolvenzverwalter und die dadurch beabsichtigte Entlastung des Insolvenzgerichts, keine weitere formelle Prüfung von Anmeldungen durch das Insolvenzgericht statt.

\section{Fehler und Ergänzung/Änderung der zulässigen Anmeldung}

Lediglich fehlerhaft bzw. unvollständig, aber grundsätzlich zulässig ist eine Insolvenzforderungsanmeldung z.B. dann, wenn ein Beweisstück, aus dem sich die Insolvenzforderung ergeben soll, nicht mit eingereicht wurde, ${ }^{282}$ die übrigen formellen Mindestanforderungen an die Anmeldung aber erfüllt sind. Fraglich ist jedoch, bis wann der Insolvenzgläubiger unter welchen Voraussetzungen und Folgen Änderungen und/oder Ergänzungen seiner Anmeldung gegenüber wem vornehmen kann.

282 Siehe unter D.IV.4. (S.49f.). 


\section{a) Vor Ablauf der Anmeldefrist}

Änderung und Ergänzungen der formell ordnungsgemäßen Anmeldung durch den Insolvenzgläubiger, etwa mit dem Hinweis, dass der angemeldete Anspruch auf einer vorsätzlich begangenen unerlaubten Handlung beruhen soll oder die angemeldete Insolvenzforderung bereits tituliert ist, sind bis zum Ablauf der Anmeldungsfrist beim zuständigen Insolvenzverwalter grundsätzlich zulässig $^{283}$ und in der Tabelle entsprechend festzuhalten. ${ }^{284}$ Nach Ablauf der Anmeldefrist wird wie nachfolgend im Einzelfall jedoch zu unterscheiden sein.

\section{b) Nach Ablauf der Anmeldefrist bis zur Feststellung der Forderung}

Ermäßigt der Gläubiger seine Forderung, ${ }^{285}$ ergänzt er den Rechtsgrund oder schiebt weitere Anspruchsgrundlagen nach, so ist dies dann unschädlich, wenn hierdurch keine Änderung des Schuldgrundes seiner angemeldeten Forderung oder des ihr zugrunde liegenden Lebenssachverhalts - vergleichbar der Klageänderung gem. § 263 ZPO eintritt. ${ }^{286}$ Das Verbleibende bzw. Grundlegende ist dann bereits in der ursprünglichen Anmeldung enthalten, so dass eine andere und damit neue rechtliche Würdigung und Prüfung durch die Verfahrensbeteiligten gerade nicht erforderlich ist.

Wird der Forderungsbetrag daher erhöht oder der Anspruchsgrund an sich ausgetauscht bzw. der zugrunde liegende Lebenssachverhalt geändert/erweitert, so stellt dies eine wesentliche Änderung dar, fällt damit unter eine nachträgliche Änderung i.S.d. § 177 I 3 InsO und ist demzufolge wie eine nachträgliche Anmeldung gem. $\S 177 \quad \mathrm{I} \quad \mathrm{InsO}^{287} \quad \mathrm{zu}$

283 Nerlich/Römermann/Becker, § 174 Rn.18ff.; Braun/Specovius, § 174 Rn.30; Kübler/Prütting/Bork/Pape, § 174 Rn.32; HHK/Preß/Henningsmeier, § 174 Rn.28; FK/Kießner, § 174 Rn.22; Uhlenbruck/Uhlenbruck, § 174 Rn. 23 m.w.N.

284 Siehe auch D.V.4. (S.89f.).

285 BGH ZIP 1988, 229 (229f.); zur Geltendmachung eines Mehrbetrags siehe auch BFH BStBl 1953 III, 364 (365).

286 BGH KTS 1973, 266 (269); Kübler/Prütting/Bork/Pape, § 181 Rn.2; MüKoInsO/Nowak, § 174 Rn.16; Nerlich/Römermann/Becker, $\S 174$ Rn.20; Gottwald/Eickmann, § 63 Rn.31ff.; Uhlenbruck/Uhlenbruck, § 174 Rn.23; Braun/Specovius, § 174 Rn.31.

287 Siehe auch E.II. (S.187ff). 
behandeln. ${ }^{288}$ Dies geschieht bis zur Feststellung der Insolvenzforderung in der Regel durch Rücknahme der ursprünglichen Anmeldung und Neuanmeldung mit dem nunmehrigen Forderungs- bzw. Anmeldungsinhalt gegenüber dem Insolvenzverwalter. ${ }^{289}$ Die Begriffe „Neuanmeldung“ und „nachträgliche Anmeldung“ werden generell deckungsgleich verwendet. Den Verfahrensbeteiligten muss in diesen Fällen nämlich erneut die Möglichkeit gegeben werden, die Forderung zu prüfen und gegebenenfalls zu widersprechen, da sich der Umfang der Einflussnahme des (Erneut-/ Erweitert-)Anmeldenden auf das Verfahren gegenüber den übrigen Verfahrensbeteiligten ändern/erweitern kann; sei es etwa hinsichtlich einer Erweiterung des Stimmrechts durch einen erhöhten Forderungsbetrag (§ 76 II InsO) oder hinsichtlich der Nachhaftung des Schuldners durch Anmeldung des Forderungsattributs der vorsätzlich begangenen unerlaubten Handlung (§ 302 Nr.1 InsO).

Fraglich ist, ob bzw. inwieweit der Wechsel des Forderungsinhabers - egal ob durch Einzel- oder Gesamtrechtsnachfolge - eine (wesentliche) Änderung der Forderungsanmeldung darstellt. Im Fall eines Gläubigerwechsels ist danach zu unterscheiden, ob die Rechtsnachfolge streitig oder unstreitig ist. Bei einer unstreitigen Rechtsnachfolge ist der Tabelleneintrag lediglich $\mathrm{zu}$ berichtigen, ${ }^{290}$ und die Rechtsnachfolge je nach Verfahrensstand durch Erbschein, Abtretungsurkunde etc. gegenüber dem Insolvenzverwalter bzw. dem Insolvenzgericht nachzuweisen. ${ }^{291}$ Der Rechtsnachfolger tritt dann ohne weiteres an die Stelle des Anmelders. ${ }^{292}$ Es bedarf damit keiner Neuanmeldung nach § 177 InsO. Ist die Nachfolge jedoch streitig, muss der angebliche Rechtsnachfolger neben dem

288 Beck/Depré/Ringstmeier, § 11 Rn.197; Uhlenbruck/Uhlenbruck, § 174 Rn.23; HHK/Preß/Henningsmeier, § 174 Rn.28; MüKo-InsO/Nowak, § 174 Rn.16; Gottwald/Eickmann, § 63 Rn.31ff.; Nerlich/Römermann/Becker, § 174 Rn.19; Braun/Specovius, § 174 Rn.31.

289 Uhlenbruck/Uhlenbruck, § 177 Rn.6; Gottwald/Eickmann, § 63 Rn.32.

290 Dazu auch unter D.V.4. (S.89f.).

291 Ist die Forderung bereits tituliert, so ist der Vollstreckungstitel gem. §§ 727, 731 ZPO umzuschreiben und gem. § 750 I 2 ZPO dem Insolvenzverwalter/-gericht (erneut) zuzustellen.

292 MüKo-InsO/Nowak, § 177 Rn.12; Braun/Specovius, $\S 177$ Rn.28; GrafSchlicker/Graf-Schlicker, § 177 Rn.11; Uhlenbruck/Uhlenbruck, § 177 Rn.6; FK/Kießner, § 177 Rn.37; Kuhn/Uhlenbruck, KO, § 142 Rn.3c; Kilger/K.Schmidt, KO, § 142 Rn.4. 
Erstanmelder die Forderung erneut anmelden. ${ }^{293}$ Dadurch nimmt er selbst am Verfahren teil, kann der Forderungsberechtigung des anderen im Prüfungstermin widersprechen und die Klärung der wahren Forderungsinhaberschaft im Feststellungsprozess gemäß den §§ 179ff. InsO betreiben. Die Forderungsanmeldung ist dann mit dem Hinweis in die Tabelle einzutragen, dass dieselbe Forderung für verschiedene Anmelder in Anspruch genommen wird. Dies entspricht der bereits dargestellten Behandlungsweise von Doppelanmeldungen auf die an dieser Stelle verwiesen wird. ${ }^{294}$

\section{c) Nach Feststellung der Insolvenzforderung}

Nach der Feststellung der Insolvenzforderung ist eine Rücknahme der Anmeldung aufgrund der Feststellungswirkung des § 178 III InsO nicht mehr möglich, ${ }^{295}$ so dass für eine Ergänzung der angemeldeten und insoweit bereits festgestellten Insolvenzforderung hinsichtlich einer bis dahin noch nicht angebrachten Insolvenzforderungseigenschaft grundsätzlich eine erweiternde nachträgliche Änderungsanmeldung erfolgen muss (§ 177 I 3 InsO). Hierunter fällt insbesondere die zusätzliche Qualifizierung einer bereits festgestellten Insolvenzforderung als eine Forderung aus vorsätzlich begangener unerlaubter Handlung des Schuldners. Von der Wirkung der bereits erfolgten Feststellung gem. $\S 178$ III InsO kann materiellrechtlich nämlich nur das umfasst sein, ${ }^{296}$ was zuvor Gegenstand der Feststellung war. ${ }^{297}$ Verfahrenstechnisch ist das Privileg des Insolvenzgläubigers einer Forderung aus vorsätzlich begangener unerlaubter Handlung gem. §§ 174 II, 302 Nr.1 InsO wie ein Konkursvorrecht alten Rechts zu behandeln (§ 142 II KO), ${ }^{298}$ was bedeutet, dass dieser Rechtsgrund auch für eine bereits zur Tabelle festgestellte

293 BGH NJW 1997, 1014 (1015); Braun/Specovius, § 177 Rn.30; MüKoInsO/Nowak, § 177 Rn.12; Uhlenbruck/Uhlenbruck, § 177 Rn.6; Graf-Schlicker/Graf-Schlicker, § 177 Rn.12; FK/Kießner, § 177 Rn.37, 40; Kuhn/Uhlenbruck, KO, § 142 Rn.3c; Kilger/K.Schmidt, KO, § 142 Rn.4.

294 Siehe schon D.IV.2.d) (S.43f.); weiter BGH NJW 1997, 1014 (1015); MüKoInsO/Nowak, § 177 Rn.12.

295 Siehe dazu ausführlich unter D.VII. (S.121ff.).

296 Siehe dazu unter E.III.2. (S.208ff.).

297 BGH ZinsO 2008, 325 (327) m.w.N.

298 Begr. zu Nr.12 (§ 174 InsO) RegE, BT-Drucks. 14/5680, S.27. 
Insolvenzforderung noch nachträglich beansprucht und mit einer Änderungsanmeldung gem. § 177 I 3 InsO in das Insolvenzverfahren eingeführt werden kann. ${ }^{299}$ Die Prüfung dieses nachträglich angemeldeten Rechtsgrundes und ein daraufhin $\mathrm{zu}$ erhebender Widerspruch, obliegen jedoch ausschließlich dem Schuldner und den in der Nachhaftung miteinander konkurrierenden Insolvenzgläubigern. ${ }^{300}$ Solche nachträglichen Anmeldungen können jedoch nur bis zum Ende des Schlusstermins vorgenommen werden, worauf im weiteren Verlauf der Arbeit aber noch einzugehen sein wird. ${ }^{301}$

Fraglich ist jedoch, wie die Situation zu beurteilen ist, wenn die Rechtsnachfolge bzw. (Mit-)Inhaberschaft einer inzwischen zur Tabelle festgestellten Forderung streitig wird. In diesem Fall ist nämlich - anders als bei der Nachtragsanmeldung des Forderungsattributs - die Inhaberschaft als notwendiger Bestandteil des Forderungsgrundes/ Lebenssachverhaltes bereits Gegenstand der festgestellten Insolvenzforderung. Die nachträgliche Anmeldung des die insoweit bereits festgestellte Insolvenzforderung beanspruchenden Gläubigers ist zwar unter Einhaltung der formellen Voraussetzungen grundsätzlich möglich, würde jedoch seinem eigentlichen Begehren, die Position des derzeitig festgestellten Insolvenzgläubigers zu übernehmen, nicht weiterhelfen. Zum einen kann der vermeintliche Gläubiger keinen Widerspruch mehr gegen die bereits festgestellte Insolvenzforderung erheben, ${ }^{302}$ wie dies vor der Feststellung möglich (gewesen) wäre, um diese dem Feststellungsverfahren der $\S \S 179 f f$. InsO zuzuführen. ${ }^{303}$ Zum anderen würde seiner eigenen Anmeldung unter Bezugnahme auf den bereits zur Tabelle festgestellten Insolvenzforderungsinhabers widersprochen werden. Sein Vorgehen gegen diesen Widerspruch, mit dem Ergebnis einer zusätzlichen und damit doppelten Feststellung der(selben) Insolvenzforderung zur Tabelle, kann aufgrund der zum Zeitpunkt der Prüfung anderweitig festgestellten Inhaberschaft ebenfalls keinen Erfolg haben. Der Weg des Anmelde- und Feststellungsverfahrens der §§ 174, 179ff. InsO mit einer nachträglichen Anmeldung über die für sich selbst

\footnotetext{
BGH ZinsO 2008, 325 (327).

Siehe dazu ausführlich unter E.I.4.c)cc) (S.148ff.).

Siehe unter E.II.3.a) (S.192ff.).

Siehe unter E.I.4.b) (S.142ff.).

Siehe bereits unter D.IV.2.d) (S.43f.).
} 
beanspruchte, aber bereits für einen anderen Gläubiger festgestellte Insolvenzforderung ist für den vermeintlichen Gläubiger somit nicht (mehr) erfolgreich gangbar. Schließlich geht es im vorliegenden Fall auch nicht mehr um die berechtigte Teilnahme der Insolvenzforderung am Insolvenzverfahren im Verhältnis zu den anderen Insolvenzgläubigern. Dieser Teilnahmeberechtigung wurde schließlich mit der grundsätzlichen Feststellung der Insolvenzforderung bereits entsprochen, so dass es sich nicht mehr um eine insolvenzrechtliche, sondern um eine hiervon unabhängige und damit richtigerweise außerhalb des Insolvenzverfahrens zu verfolgende (materiellrechtliche) Streitigkeit geht, nämlich der grundsätzlichen Forderungsinhaberschaft zwischen zwei Gläubigern (Prätendenten). ${ }^{304}$ Das Feststellungsinteresse ist damit nunmehr ein anderes als noch im Feststellungsverfahren der §§ 174ff. InsO, in dem es zwischen den Streitenden zugleich auch um die Feststellung der Forderung als Insolvenzforderung $\mathrm{zu}$ eigenen Gunsten ging. Die beanspruchte Inhaberschaft der Forderung ist nunmehr das einzige Interessensziel. Die Insolvenzgläubigerstellung ist lediglich die sich aus der Inhaberschaft der - in diesem Fall nämlich bereits festgestellten - Forderung ergebende Folge ( $§ 38$ InsO). ${ }^{305}$ Daher wird der die Insolvenzforderung beanspruchende Gläubiger eine allgemeine Feststellungsklage gem. § 256 ZPO auf Feststellung seiner Inhaberschaft gegenüber dem in der Tabelle eingetragenen Insolvenzgläubiger erheben können. Hierdurch wird die Berechtigung der Insolvenzforderung nach Grund und Höhe im Verhältnis zu den festgestellten Insolvenzforderungen der übrigen Insolvenzgläubiger nicht berührt und unterläuft somit auch nicht den Sinn und Zweck der $\S \S 174$ I, 178 III InsO, eine eindeutige und feststehende Bindungswirkung innerhalb der Insolvenzgläubigergemeinschaft hinsichtlich der berechtigten Teilnahme der Insolvenzforderung zu schaffen. ${ }^{306}$ Wer letztlich Inhaber der Insolvenzforderung ist, hat auf die entscheidende Quote schließlich keinen Einfluss. Wenn der Feststellungsklage stattgegeben wird, kann der Berechtigte nunmehr die Berichtigung der Tabelle zu seinen Gunsten unter

\footnotetext{
304 Siehe bereits unter D.IV.2.d) (S.43f.). Vgl. auch BGH NJW 1997, 14014 (1015): „Schuldrechtliche Sonderbeziehung zwischen den Gläubigern sind außerhalb des Konkursverfahrens zu klären, wenn die Verteilung der Masse insgesamt nicht zwangsläufig davon abhängt.“ Siehe bereits unter C.I. (S.10ff.).

Siehe auch Fn.471, S.121.
} 
Vorlage des erstrittenen Titels gegen den bisher eingetragenen Gläubiger vornehmen lassen. Um eine Verteilung während des Prätendentenstreits auf die berechtigte und $\mathrm{zu}$ berücksichtigende Insolvenzforderung $\mathrm{zu}$ Ungunsten des Obsiegenden zu verhindern, sollte der Prätendentenstreit dem Insolvenzverwalter umgehend angezeigt und der Anteil auf die Insolvenzforderung in entsprechender Anwendung des $\S 189$ I, II InsO bis zu einer Entscheidung zurückbehalten werden können. Nachteile sind hiermit nicht verbunden.

Sämtliche Ergänzungs- oder Änderungsanträge sind grundsätzlich beim Insolvenzverwalter anzubringen, der über ihre rechtliche Einordnung und Behandlung im Verfahren $\mathrm{zu}$ entscheiden hat und entsprechend notwendige Korrekturen oder Ergänzungen in der Tabelle vor ihrer Niederlegung beim Insolvenzgericht selbst vornimmt bzw. nach Niederlegung vom Urkundsbeamten der Geschäftsstelle des Insolvenzgerichts vornehmen lässt. ${ }^{307}$ Sollten entsprechende Anträge beim Insolvenzgericht angebracht werden, sind diese an den Insolvenzverwalter weiterzuleiten.

\section{Anmeldefrist}

Mit der Eröffnung des Insolvenzverfahrens bestimmt das Insolvenzgericht im Eröffnungsbeschluss gem. § 29 I Nr.2 InsO die Frist, in der die Insolvenzforderungen bei dem Insolvenzverwalter unter Beachtung des $\S 174$ InsO anzumelden sind (§ 28 I InsO). Gleichzeitig wird der Termin für eine Gläubigerversammlung festgelegt, in der die Insolvenzforderungen im Prüfungstermin geprüft werden. Auf die eventuelle Anmeldungsmöglichkeit nachrangiger Forderungen (§ 39 InsO) ist gem. $\S 174$ III InsO vom Gericht besonders hinzuweisen. ${ }^{308}$ Die Anmeldefrist ist dabei auf einen Zeitraum von mindestens zwei Wochen und höchstens drei Monaten festzusetzen ( $§ 28$ I 2 InsO). Der Prüfungstermin ist im Zusammenhang mit der Anmeldefrist entsprechend dem zu erwartenden Verfahrensumfang festzulegen. Der Zeitraum zwischen Ablauf der Anmeldefrist und dem Prüfungstermin soll grundsätzlich mindestens eine

Siehe dazu unter D.V.2. (S.85ff.).

308 Siehe schon C.III. (S.20ff.). 
Woche, höchstens jedoch zwei Monate ${ }^{309}$ betragen (§ 29 I Nr.2 InsO). Neben der obligatorischen Verbindung von Berichts- und Prüfungstermin gem. § 29 II InsO wird bei Eröffnung eines Verbraucherinsolvenzverfahrens abweichend von $\S 29$ InsO nur der Prüfungstermin bestimmt (§ 312 I 2 InsO).

Die Festlegung der Anmeldefrist dient damit der ersten Einteilung des Insolvenzverfahrens in die Verfahrensabschnitte des Anmeldeverfahrens einerseits und des weiterführenden Prüfungs- und Feststellungsverfahrens andererseits. Gleichzeitig wird durch die Anmeldefrist der Zeitpunkt festgelegt, ab dem eine angebrachte Anmeldung als verspätet gilt und die Regelungen der nachträglichen Anmeldung des $§ 177$ InsO zur Anwendung kommen. Die Regelung des § 177 I 1 InsO für nachträgliche Anmeldungen stellt dabei klar, dass die Anmeldefrist keine Ausschlussfrist ist, ${ }^{310}$ wie es noch bei der Anmeldefrist des $§ 5$ S.2 Nr.3 GesO im Hinblick auf $\S 14$ I 1 GesO der Fall war. Jene Regelung des § 14 I 1 GesO, wonach verspätete Anmeldungen im Insolvenzverfahren nur dann berücksichtigt wurden, wenn sie unverschuldet waren und das Gericht zugestimmt hat, wurde nicht übernommen. ${ }^{311}$ Ein Insolvenzgläubiger, der die Frist ungenutzt verstreichen lässt, ist daher nicht präkludiert, sondern kann gleichwohl noch am Verfahren teilnehmen und seine Insolvenzforderungen auch nach Ablauf der Anmeldefrist (vgl. § 177 InsO) und sogar nach dem Prüfungstermin bis zum Schlusstermin des Verfahrens anmelden. ${ }^{312}$ Gleichwohl sind, im Gegensatz zu der fristgerecht angemeldeten Insolvenzforderung, mit einer verspäteten Anmeldung entsprechende Nachteile verbunden, da das Verstreichenlassen der Anmeldefrist - je nach

309 In Großverfahren soll eine Überschreitung der zwei Monatsfrist des § 29 I Nr.2 InsO als Sollvorschrift und in Hinblick auf die für die Prüfung der angemeldeten Insolvenzforderungen erforderliche Zeit nach allgemeiner Auffassung möglich sein. - MüKo-InsO/Schmahl, §§ 27-29 Rn.50; Jaeger/Schilken, InsO, § 28 Rn.8; HK/Kirchhof, § 29 Rn.6 m.w.N. Siehe auch unter E.I.4.d)bb) (S.160ff.).

310 Jaeger/Schilken, InsO, § 28 Rn.9; BK/Goetsch, § 28 Rn.23; MüKo-InsO/Nowak, $\S 174$ Rn.7; Hess, InsO, § 28 Rn.9; Mohrbutter/Ringstmeier/Ernestus, § 11 Rn.2; Nerlich/Römermann/Becker, § 174 Rn.9; Gottwald/Eickmann, § 63 Rn.49.

311 Aufgrund der zahlreichen Beschwerdeverfahren ausgeschlossener Gläubiger ist, insbesondere vor dem Punkt der Verfahrensbeschleunigung und -vereinfachung, die Abkehr von § 14 I GesO gerechtfertigt. - Vgl. Begr. zu § 204 (§ 177 InsO) RegE, BT-Drucks. 12/2443, S.184; Kübler/Prütting/Bork/Pape, § 177 Rn.1f.; KS/Eckardt, S.758 Rn.29; vgl. auch KO-Motive, S.357 = Hahn, KO, S.322.

312 Der Insolvenzgläubiger muss unter Umständen dann jedoch damit rechnen, kostenpflichtig in einen gesonderten Prüfungstermin oder in das schriftliche Verfahren verwiesen zu werden ( $\S 177$ I 2 InsO). Siehe dazu unter E.II.3.a) (S.192ff.). 
Verfahrensstand, aber doch regelmäßig ${ }^{313}$ - zu einer späteren Teilnahme am Insolvenzverfahren und damit zu einer späteren Einflussmöglichkeit des Anmeldenden auf das Insolvenzverfahren führt. Zudem hat der Verspätete für einen eventuell zusätzlich anzuberaumenden Prüfungstermin die Kosten zu tragen (§ 177 I 2 InsO). Eine ausführliche Erörterung nachträglicher Anmeldungen im Insolvenzverfahren wird noch an gegebener Stelle der Arbeit zu erfolgen haben. ${ }^{314}$

Als Gegenstück zur verspäteten Anmeldung einer Insolvenzforderung ist die Frage aufzuwerfen, inwieweit auch eine verfrühte Anmeldung zum Insolvenzverfahren zuzulassen ist. Zu verfrühten Anmeldungen kommt es durch die frühzeitige Zusammenarbeit im Rahmen der Tätigkeit des vorläufigen Insolvenzverwalters (§ 22 InsO) und des Schuldners mit den späteren Insolvenzgläubigern im Vorfeld des Insolvenzverfahrens und ist daher eng mit der aufgeworfenen Fragestellung verbunden.

Die Anmeldung der Insolvenzforderungen zur Tabelle ist grundsätzlich erst nach Eröffnung des Verfahrens möglich (§ 28 I InsO). Dieser Umstand und eine entsprechende Fristbestimmung im Eröffnungsbeschluss sollten den vorläufigen Insolvenzverwalter jedoch nicht zwingen, eine bereits zuvor angebrachte und damit eigentlich verfrühte Anmeldung schon im Eröffnungsverfahren als unzulässig zurückweisen zu müssen. Zwar beschränken sich die Befugnisse und Tätigkeiten eines vom Gericht bestellten vorläufigen Insolvenzverwalters (§ 21 II Nr.2 InsO) vorerst auf jene Tätigkeiten, die in $\S 22$ InsO genannt sind, doch führen schon diese dazu, den Stand der Verbindlichkeiten des Schuldners im Hinblick auf eine möglicherweise zu erwartende Verfahrenseröffnung zu ermitteln und zu diesem Zweck auch mit den - späteren - Insolvenzgläubigern Kontakt aufzunehmen. Für den Insolvenzverwalter wird dadurch frühzeitig ersichtlich sein, ob das Insolvenzgericht das Insolvenzverfahren eröffnen wird oder nicht. Gleichzeitig hindern ihn seine beschränkten Befugnisse und Tätigkeiten als vorläufiger Insolvenzverwalter nicht daran, bereits im Eröffnungsverfahren erfolgte Anmeldungen zu prüfen. Vielmehr weist $\S 174$ I 1 InsO dem Insolvenzverwalter allgemein - und damit auch dem vorläufigen Insolvenzverwalter im Eröffnungsverfahren - die

313 Siehe auch unter E.I.1. (S.128f.) und E.II.2. (S.189ff.).

314 Siehe auch unter E.II. (S.187ff.). 
Zuständigkeit für Anmeldungen zu. Dadurch kann bzw. wird sich der vorläufige Insolvenzverwalter grundsätzlich auch in diesem Verfahrensstadium bereits umfassend mit der Zulässigkeit einer Anmeldung befassen (müssen). Dies zumindest dann, wenn für ihn absehbar ist, dass das Insolvenzverfahren durch das Insolvenzgericht zeitnah eröffnet werden wird und die Zulässigkeit der ansonsten ordnungsgemäß angebrachten Anmeldung einzig von diesem Zeitpunkt abhängt. Denn dann würde es sowohl für den (vorläufigen) Insolvenzverwalter als auch für den verfrüht anmeldenden Gläubiger einen prozessökonomisch unsinnigen Formalismus darstellen, den Gläubiger nach der Eröffnung des Insolvenzverfahrens zu einer Wiederholung der bereits vorliegenden, verfrühten Anmeldung beim nunmehrigen Insolvenzverwalter aufzufordern, ${ }^{315}$ insbesondere weil beide regelmäßig personenidentisch sein werden. Selbst wenn die Insolvenzverwalter nicht identisch sein sollten, schadet auch dies nicht, da sämtliche bis dahin zusammengetragene Unterlagen und damit auch die verfrühten Anmeldungen an den nunmehr eingesetzten Insolvenzverwalter übergeben werden. Durch dieses erleichterte Vorgehen des Insolvenzverwalters wird auch die Rechtssicherheit und Rechtsklarheit nicht gefährdet, ${ }^{316}$ weil in diesem Fall der Anmeldezeitpunkt durch den Eröffnungsbeschluss eindeutig fixiert werden kann. ${ }^{317}$ Daher sollte es dem Insolvenzverwalter trotz eindeutiger gesetzlicher Bestimmung möglich sein, auch eine verfrühte Anmeldung, soweit sie ansonsten sämtlichen formellen Anforderungen entspricht, für einen zügigen Verfahrensfortgang als wirksame Anmeldung zuzulassen und in die Tabelle aufzunehmen. ${ }^{318}$ Weist

315 Hess, InsO, § 28 Rn.7; FK/Schmerbach, § 28 Rn.6; Hess, InsO, § 28 Rn.7f.; Jaeger/Weber, KO, § 138 Rn.3; Kilger/K.Schmidt, KO, § 138 Rn.2; Jaeger/Schliken, InsO, § 28 Rn.10 m.w.N.; siehe auch OLG Brandenburg ZIP 1994, 1288 (1288f.) zur GesO.

316 Unbegründet daher die Kritik bei FK/Kießner, § 174 Rn.5 und LSG BadenWürttemberg KTS 1985, 566 (568f.). Wobei im genannten Urteil zudem nicht hinreichend zwischen bloßer Anmeldung und deren Wirkung und dem nachfolgenden Prüfungsverfahren unterschieden wird.

317 Siehe auch unter D.VI.4. (S.115ff.).

318 Das Anmelderisiko bei einer verfrühten Anmeldung trägt dabei jedoch der Insolvenzgläubiger, der sich daher in jedem Fall zu versichern hat, spätestens durch Einsicht in die niedergelegte Tabelle (§ 175 I 2 InsO), dass seine Forderung auch tatsächlich in die Tabelle aufgenommen worden ist. Sollte dies nicht der Fall sein, bleibt dem Gläubiger immer noch die nachträglich Anmeldung gem. § 177 InsO. 
der Insolvenzverwalter die Anmeldung aufgrund der eindeutigen Verfahrensregelung (§ 28 I InsO) dennoch zurück, so ist dies dem Anmeldenden mit Hinweis auf seine verfrühte Anmeldung mitzuteilen. 


\section{Die Eintragung in die Tabelle}

Die Insolvenztabelle dient dazu, die wirksam angemeldeten Insolvenzforderungen $\mathrm{zu}$ katalogisieren und damit für das weitere Verfahren übersichtlich zu machen. ${ }^{319}$ Sie ist insbesondere Grundlage des späteren Prüfungstermins ( $\S \S 176$ S.1, 178 II 1, III InsO) und dient den Verfahrensbeteiligten zusammen mit den übrigen eingereichten Unterlagen dazu, sich im Vorfeld ein Bild über die Teilnahmeberechtigung der angemeldeten Insolvenzforderungen $\mathrm{zu}$ machen, um entscheiden $\mathrm{zu}$ können, wie sie sich im Prüfungstermin den einzelnen Insolvenzforderungen gegenüber verhalten wollen. ${ }^{320}$

Im Folgenden soll daher zunächst der Inhalt der Tabellenerstellung und die Bedeutung der Niederlegung der Tabelle für das weitere Verfahren aufgezeigt und erörtert werden, um Rückschlüsse auf die Aufgabenverteilung zwischen dem Insolvenzverwalter und dem Insolvenzgericht ziehen zu können. Dabei sind insbesondere die mit der Hinweispflicht des Insolvenzgerichts gem. § 175 II InsO zusammenhängenden Fragen aufzuwerfen, ab wann wem die Tabellenführung obliegt und inwiefern eine Tabellenauslegung anderorts als dem Insolvenzgericht möglich ist.

\section{Tabellenerstellung}

Gem. § 175 I 1 InsO hat der Insolvenzverwalter jede angemeldete Insolvenzforderung mit den in $\S 174$ II, III InsO genannten Angaben in eine Tabelle einzutragen. Nach $\S 175$ I 2 InsO ist die Tabelle sodann mit den Anmeldungen sowie den beigefügten Urkunden innerhalb des ersten Drittels des Zeitraums, der zwischen dem Ablauf der Anmeldefrist und dem Prüfungstermin liegt (vgl. § 29 I Nr.2 InsO), in der Geschäftsstelle des Insolvenzgerichts zur Einsicht der Beteiligten niederzulegen.

Hieraus ergibt sich, dass bis zur Niederlegung der Tabelle beim zuständigen Insolvenzgericht (§§ 2, 3 InsO) ausschließlich der Insolvenz-

319 Unter anderem auch für die Bestimmung der einzelnen Stimmrechte nach der Höhe der Forderungen, vgl. §§ 77 II, III i.V.m. 76 II InsO.

320 MüKo-InsO/Nowak, § 175 Rn.1; Nerlich/Römermann/Becker, § 175 Rn.6. 
verwalter bzw. der Sachwalter in der Eigenverwaltung oder der Treuhänder im vereinfachten Verfahren für die Führung der Tabelle zuständig ist. Dabei sind hinsichtlich der späteren Ausstellung eines Vollstreckungstitels (§ 201 II 3 InsO) alle vollstreckungsrechtlich relevanten Gläubigerdaten der wirksamen Anmeldungen unter laufender Nummer in der Reihenfolge der Anmeldungen in die Tabelle aufzunehmen und zwar so, wie sie sich aus den Anmeldungen selbst ergeben. Die Korrektur der gemachten Angaben kraft eigener Einsicht ist dem Insolvenzverwalter nicht gestattet. $^{321}$ Klarstellungen bedürfen seiner Rückfrage oder einer Erörterung im Prüfungstermin. ${ }^{322}$

Zwingend einzutragen sind dabei der Gläubiger sowie dessen evtl. Vertreter, der Grund und der Betrag der einzelnen Insolvenzforderung, ${ }^{323}$ bei nachrangigen Insolenzforderungen der Hinweis auf den Nachrang, sowie die Rangstelle und der zusätzliche explizite Vermerk, wenn sich die angemeldete Forderung aus einer vorsätzlich begangenen unerlaubten Handlung des Schuldners ergeben soll. Des Weiteren hat der Insolvenzverwalter wegen der verjährungshemmenden Wirkung (§ 204 I Nr.10 BGB) den Zeitpunkt der wirksamen Forderungsanmeldung zu vermerken. ${ }^{324}$ Die Tabelle hat weiterhin Vermerkungsmöglichkeiten/ Spalten für die Eintragung der Prüfungsergebnisse (Feststellung, Widersprüche, Stimmrecht), von Berichtigungen oder Änderungen der Anmeldung und sonstigen Anmerkungen vorzusehen. ${ }^{325} \S 5$ III InsO stellt klar, dass die Tabelle selbstverständlich mit Hilfe von EDV ${ }^{326}$ erstellt und geführt werden kann.

321 FK/Kießner, § 175 Rn.2; Nerlich/Römermann/Becker, § 175 Rn.3.

322 Siehe dazu auch E.I.3. (S.137ff.). - Unbenommen hiervon ist der Insolvenzverwalter jedoch gehalten, anhand der ihn erreichenden Anmeldungen Ergänzungen oder Verbesserungen in dem nach seinen Kenntnissen geführten Gläubigerverzeichnis (§ 152 I Fall 3 InsO) anzubringen, was sich dann auch in der Vermögensübersicht (§ 153 I 1 InsO) widerspiegelt.

323 So schon zum Urkundsbeamten KO-Motive, S.360 = Hahn, KO, S.324.

324 HK/Irschlinger, § 175 Rn.3; MüKo-InsO/Nowak, § 175 Rn.2ff.; Graf-Schlicker/GrafSchlicker, § 175 Rn.2; Braun/Specovius, § 175 Rn.3.

325 MüKo-InsO/Nowak, § 175 Rn.2ff.; Uhlenbruck/Uhlenbruck, $\S 175$ Rn.9; Gottwald/Eickmann, § 63 Rn.18; siehe auch KO-Motive, S.360 = Hahn, KO, S.324.

326 Z.B. „Winsolvenz“ der Firma STP AG, Karlsruhe. 


\section{Niederlegung der Tabelle}

Innerhalb des ersten Drittels des Zeitraums zwischen Ablauf der Anmeldefrist und dem Prüfungstermin geht die Tabellenführung durch Niederlegung der Tabelle in der Geschäftsstelle des ausschließlich zuständigen Insolvenzgerichts (vgl. § 3 InsO) auf den Urkundsbeamten der Geschäftsstelle über. ${ }^{327}$

Unter „Niederlegung“ ist der für die Verfahrensbeteiligten als Auslage einzusehende Ausdruck der Tabelle bei Gericht zu verstehen. ${ }^{328}$ Die Tabelle kann neben dem Ausdruck für ihre Weiterführung per EDV neuerdings (vgl. § 5 IV InsO) auch als elektronisches Dokument an das Gericht übergeben werden. Dies natürlich nur, soweit die verwendete Software, Datenträger und sonstige Datenübermittlungsmöglichkeiten mit denen des zuständigen Insolvenzgerichts kompatibel sind.

Nachfolgende Eintragungen kann damit nur noch das Insolvenzgericht vornehmen. $^{329}$ Etwas anderes, etwa dass die Tabelle bis zum Prüfungstermin oder überhaupt vom Insolvenzverwalter weitergeführt wird, ${ }^{330}$ kann dem Wortlaut und Sinngehalt des $\S 175$ I 2 InsO nicht entnommen werden. Die Niederlegung der Tabelle unmittelbar nach Ablauf der Anmeldefrist stellt den Abschluss des allgemeinen Anmeldeverfahrens und damit auch der Tabellenerstellung und -führung durch den Insolvenzverwalter dar. Nur durch die zentral bei Gericht ausgelegte, dort einheitlich und bei nachträglichen Anmeldungen kontinuierlich fortgeführte Tabelle wird sichergestellt, dass alle Verfahrensbeteiligten die bereits angemeldeten Insolvenzforderungen und die dazugehörigen Unterlagen eingehend auf ihre Teilnahmeberechtigung am Verfahren prüfen und darüber hinaus auch frühzeitig von nachträglichen Anmeldungen und sonstigen Änderungen Kenntnis nehmen

327 Kübler/Prütting/Bork/Pape, § 175 Rn.1b; Graf-Schlicker/Graf-Schlicker, § 175 Rn. 11; Hess, InsO, § 175 Rn.15; Gottwald/Eickmann, § 63 Rn.34; KS/Eckardt, S.752 Rn.16.

328 Ob die Darstellung der EDV geführten Tabelle lediglich per Bildschirm, auf dem diese abgelesen werden kann, für eine Niederlegung ausreicht, erscheint mangels Regelung und auch wegen der Unübersichtlichkeit dieses Mediums gerade in größeren Verfahren als äußerst zweifelhaft und ist daher abzulehnen. So aber Nerlich/Römermann/Becker, § 175 Rn.11; wohl auch HK/Eickmann, $\S 175$ Rn.12.

329 Siehe auch E.II.1. (S.188f.).

330 So etwa FK/Kießner, § 175 Rn.9; Braun/Specovius, § 175 Rn.4; widersprüchlich Uhlenbruck/Uhlenbruck, § 175 vgl. Rn.8 und Rn.3. 
können. ${ }^{331}$ Zudem kann das Insolvenzgericht nur auf diese Weise frühzeitig von angemeldeten Insolvenzforderungen aus vorsätzlich begangener unerlaubter Handlung erfahren und entsprechend zeitnah und verfahrensplanend seiner Hinweispflicht gegenüber dem Schuldner gem. § 175 II InsO nachkommen. ${ }^{332}$ Dies wäre durch eine Weiterführung des Insolvenzverwalters nicht sichergestellt. Vielmehr hätte dessen Weiterführung bis zum Prüfungstermin und darüber hinaus eine nachträgliche - durch Abgleichung evtl. sogar fehlerhafte - Ergänzung bzw. zweite Niederlegung der eigentlich schon vorliegenden Tabelle zur Folge. ${ }^{333}$ Dies sieht die Insolvenzordnung aber gerade nicht vor. Denn die Zuständigkeit für die Anmeldungen und die damit einhergehende Tabellenerstellung wurde zur Entlastung der Gerichte auf den Insolvenzverwalter übertragen. ${ }^{334}$ Nach Abschluss des allgemeinen Anmeldeverfahrens durch Niederlegung der erstellten Tabelle ist dieses Ziel erreicht. Die weit weniger zeit- und verwaltungsaufwendige Fortführung der Tabelle im Rahmen ihrer Ergänzung im weiteren Prüfungs- und Feststellungsverfahren durch Widerspruchs-/ Feststellungsvermerke und sonstige Änderungen und Nachträge sind ausschließlich durch das Insolvenzgericht vorzunehmen (vgl. § 178 II 1, 2 InsO). ${ }^{335}$ Hierdurch wird sichergestellt, dass das Insolvenzgericht den Gesamtüberblick über den Verfahrensstand behält und frühzeitig planungsrelevante Tatsachen - z.B. Ansetzen eines weiteren Prüfungstermins aufgrund nachträglicher Anmeldungen; Nachkommen seiner Hinweispflicht gem. § 175 II InsO - in die weitere Durchführung des Verfahrens einfließen lassen kann. Dass der Insolvenzverwalter gleichwohl weiterhin für die (nachträglichen) Anmeldungen/ Änderungsanmeldungen zuständig ist, ${ }^{336}$ beruht auf seiner umfassenden (Vor-)Kenntnis über die einzelnen Sach- und Streitstände zwischen den Insolvenzgläubigern und dem Schuldner ${ }^{337}$ und der damit eng verbundenen Prüfung der

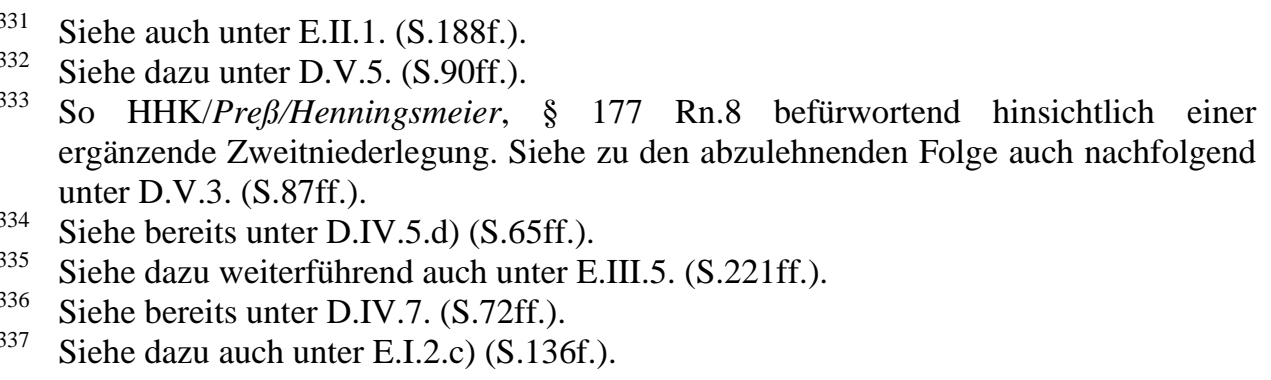


angemeldeten Insolvenzforderungen. Insofern ist hier zwischen der sicherzustellenden Verfahrensleitung und -planung durch Übertragung der Tabellenführung auf das Insolvenzgericht und der inhaltlichen Auseinandersetzung mit den einzelnen Anmeldungen und Forderungen der Insolvenzgläubiger und deren Prüfung durch den Insolvenzverwalter zu unterscheiden, so dass dem Insolvenzverwalter auch nach Sinn und Zweck der weiteren Tabellenführung diese nach Niederlegung der Tabelle nicht (mehr) zustehen kann. Für eine weiterführende Übertragung der Tabellenführung auf den Insolvenzverwalter wären zudem weitere Regelungen erforderlich, etwa zur Erteilung von Tabellenauszügen und Abschriften an Einsichtsberechtigte (vgl. § 299 ZPO i.V.m. § 4 InsO) ${ }^{338}$ und Aufbewahrungsvorschriften, ${ }^{339}$ die aber ebenfalls nicht bestehen. Deshalb bestimmen im Gegenzug auch viele Aktenordnungen der Länder ${ }^{340}$ explizit, dass die weitere Führung der dem Insolvenzgericht nach § 175 InsO vorgelegten Tabelle dem Urkundsbeamten der Geschäftsstelle obliegt. $^{341}$

\section{Niederlegungsfrist und -ort}

Die Niederlegungsfrist ist dabei abhängig von der nach § 29 I Nr.2 InsO vom Insolvenzgericht getroffenen Terminbestimmung, die zwischen einer Woche und zwei Monaten liegen kann. ${ }^{342}$ Der Tabelle beizufügen sind die Anmeldungen und die den Anmeldungen beigefügten Urkunden und Beweisstücke (§ 175 I 2 InsO).

Durch das Insolvenzgericht als Hinterlegungsstelle der Tabelle die Geschäftsräume des Insolvenzverwalters bestimmen oder schon die

Siehe dazu auch unter D.VI.3. (S.108ff.).

Siehe auch bei Hess, InsO, § 175 Rn.27 die ablehnenden Ergebnisse der BundLänder-Arbeitsgruppe hinsichtlich einer Tabellenauslegung durch den Insolvenzverwalter.

340 Gem. § 15a V 1 AktO für die Gerichte der ordentlichen Gerichtsbarkeit und die Staatsanwaltschaften (herausgegeben vom Niedersächsischen Ministerium der Justiz) obliegt „die weitere Führung der dem Insolvenzgericht nach § 175 InsO vorgelegten Tabelle...dem Urkundsbeamten der Geschäftsstelle“. - Siehe z.B. auch § 15a V 1 Aktenordnung Nordrhein-Westfalen; § $16 \mathrm{~V} 1$ Brandenburgische Aktenordnung; Bähr, InVo 1998, 205 (211); Graf-Schlicker/Graf-Schlicker, § 175 Rn.11.

341 Uhlenbruck/Uhlenbruck, § 175 Rn.8; Kübler, in: FS/Henckel, 495 (504); auch Bähr, InVo 1998, 205 (211).

342 Siehe schon D.IV.7. (S.72ff.). 
Niederlegung der Tabelle selbst beim Insolvenzverwalter durchführen zu lassen, ${ }^{343}$ ist mit dem eindeutigen Wortlaut des $\S 175$ I 2 InsO nicht vereinbar und abzulehnen. ${ }^{344}$ Jedem formellen wie materiellrechtlichen Insolvenzgläubiger muss in einem parteiöffentlichen Verfahren ${ }^{345}$ die Möglichkeit gegeben werden festzustellen, welche Insolvenzgläubiger mit welchen Forderungen am Verfahren beteiligt sind, um gegebenenfalls Informationen einzuholen, die im Prüfungstermin sodann die Insolvenzgläubiger in die Lage versetzen, die Forderungen anderer Insolvenzgläubiger $\mathrm{zu}$ bestreiten. Diese Informationsmöglichkeit im Rahmen der Akteneinsicht (§ 299 ZPO i.V.m. § 4 InsO) ${ }^{346}$ hat die Insolvenzordnung den Verfahrensbeteiligten jedoch dadurch eingeräumt, dass die Tabelle zentral und innerhalb einer gesetzlich definierten Frist auf der Geschäftsstelle des zuständigen Insolvenzgerichts zur Einsicht niedergelegt wird (§ 175 I 2 InsO). Hiermit eng verbunden ist der eben bereits dargestellte Übergang der Tabellenführung und der daran anschließenden Tätigkeiten auf das ausschließlich zuständige Insolvenzgericht (§ $3 \mathrm{InsO}$ ), der mit einer Auslegung an einem anderen Ort nicht vereinbar wäre. Relevant ist dies insbesondere im Hinblick auf die rechtzeitige Hinweispflicht des Insolvenzgerichts gem. § 175 II InsO, der nachzukommen für das Insolvenzgericht nur aus der Tabelle und den Anmeldungen ersichtlich wird. Diese frühzeitige Kenntnisnahme gilt auch für die untereinander konkurrierenden Insolvenzgläubiger von Forderungen aus vorsätzlich begangener unerlaubter Handlung, denen ausreichend Zeit für die Prüfung eines isolierten Widerspruchs gegen das Forderungsattribut einer Forderung zur Verfügung stehen muss. ${ }^{347} \mathrm{Im}$ Gegensatz zum Schuldner steht diesen Insolvenzgläubigern nämlich nicht die Möglichkeit der Wiedereinsetzung in den vorigen Stand zur Verfügung (§ 186 I 1 InsO). Auch eine zusätzliche Auslage in einem anderen Amtsoder Insolvenzgericht ${ }^{348}$ wird aufgrund der einheitlich und zentral zu führenden Tabelle daher nicht als zulässig erachtet werden können, da jede

343 So Nerlich/Römermann, § 175 Rn.8; Uhlenbruck/Uhlenbruck, § 175 Rn.11.

344 Siehe auch Graf-Schlicker/Graf-Schlicker, § 175 Rn. 11; Braun/Specovius, § 175 Rn.21f.

345 Siehe dazu unter E.I.2. (S.134f.).

346 Siehe dazu ausführlich unter D.VI.3. (S.108ff.).

347 Siehe dazu ausführlich unter E.I.4.c)cc) (S.148ff.).

348 So Nerlich/Römermann/Becker, § 175 Rn.8. Gegen eine zusätzliche Auslage spricht nicht unbedingt § 175 I 2 InsO, wohl aber § 3 InsO. 
Veränderung der „Haupttabelle“ durch ihre Erweiterung (nachträgliche Anmeldungen) oder Korrektur zu verfahrensrechtlichen Unsicherheiten bei der/den anderen Tabellen hinsichtlich einer richtigen und vor allem zeitnahen Übertragung führt. Zudem haben die mit der Tabelle eingereichten urkundlichen Beweisstücke in jedem Fall bei dem eigentlichen Insolvenzgericht $\mathrm{zu}$ verbleiben, so dass eine anderweitige Auslage dann nur durch jeweilige Kopien möglich wäre. Dem müsste hinsichtlich des Sinns und Zwecks der Auslage, nämlich der Prüfungs- und Informationsmöglichkeit der Verfahrensbeteiligten, auch vollständig nachgekommen werden. Inwieweit diese Möglichkeit einen je nach Verfahrensumfang entsprechend zusätzlichen Arbeits- und Kostenaufwand rechtfertigen mag, sei dahingestellt. Vielmehr wird die sicherzustellende Informationsmöglichkeit der Verfahrensbeteiligten über die - noch zu erörternde - Akteneinsicht selbst zu lösen sein. ${ }^{349}$

\section{Berichtigungen und Ergänzungen der Tabelle ${ }^{350}$}

Bis zu ihrer Niederlegung ist der Insolvenzverwalter ohne weiteres berechtigt und verpflichtet, die Tabelle zu berichtigen oder zu ergänzen, soweit es sich um offensichtliche Unrichtigkeiten handelt, wie z.B. Schreibfehler oder offensichtliche Abweichungen zwischen Anmeldung und Eintragung. ${ }^{351}$ Dies kann auch auf Antrag erfolgen, wenn die Unrichtigkeit der Eintragung nachgewiesen wird. ${ }^{352}$ Hiervon zu unterscheiden ist die bereits erörterte ${ }^{353}$ Änderung der Anmeldung (§ 177 I 3 InsO), die eine komplett neue Eintragung nach Rücknahme der Anmeldung erfordert bzw. nach bereits erfolgter Feststellung durch entsprechenden Hinweis in der Tabelle stets im Verbund mit der bereits bestehenden Eintragung für die entsprechende Insolvenzforderung zu sehen ist.

Siehe dazu unter D.VI.3. (S.108ff.).

Siehe ergänzend auch unter E.III.6. (S.231ff.).

Uhlenbruck/Uhlenbruck, § 175 Rn.10; MüKo-InsO/Nowak, $\S 175$ Rn.14; Braun/Specovius, § 175 Rn.14.

352 BGH ZIP 1984, 980 (981).

353 Siehe schon unter D.IV.7. (S.72ff.) zu Änderungen und Ergänzungen durch die anmeldenden Insolvenzgläubiger. 
Mit Niederlegung der Tabelle geht auch die Berichtigungsmöglichkeit auf das Insolvenzgericht über. So sind wegen der Beurkundungsfunktion (vgl. $\S 178$ II, III InsO) nachträgliche Änderungen sowohl bei Rücknahme des Widerspruchs durch den Insolvenzverwalter, durch Insolvenzgläubiger oder Schuldner als auch nach Obsiegen im Feststellungsprozess (§ 183 II InsO) oder die Berichtigung unrichtiger Feststellungsvermerke nur noch durch das Insolvenzgericht vorzunehmen. ${ }^{354}$ Entsprechende Veränderungen in der Tabelle sind dem Insolvenzverwalter dann selbstverständlich mitzuteilen.

\section{Hinweispflicht des Insolvenzgerichts bei Deliktsforderungen}

Hat ein Insolvenzgläubiger bei der Anmeldung seiner Forderung Angaben zu einer vorsätzlich begangenen unerlaubten Handlung des Schuldners gemacht und widerspricht der Schuldner nicht, so wird dieser Rechtsgrund von der Rechtskraftwirkung der Tabelleneintragung (§ 178 Abs. 3 InsO) erfasst. Damit wäre die Forderung von einer Restschuldbefreiung ausgeschlossen ( $\S 302$ Nr.2 InsO), ohne dass die schwerwiegende Konsequenz der damit verbundenen Nachhaftung dem Schuldner stets bewusst sein wird. Um dem rechtlich wenig versierten Schuldner die Konsequenzen einer solchen Forderungsanmeldung des Gläubigers zu verdeutlichen und ihn auf die Rechtsfolgen und die Möglichkeit des Widerspruchs aufmerksam zu machen, schreibt § 175 II InsO dem Insolvenzgericht daher vor, den Schuldner ausdrücklich hierauf hinzuweisen. Die Belehrung hat dabei individuell auf die einzelne Insolvenzforderung abzustellen und kann daher grundsätzlich nicht pauschal etwa in einem Antragsformular erfolgen. ${ }^{355} \mathrm{Da}$ die Anforderungen für das Insolvenzgericht jedoch beherrschbar bleiben müssen, sollte ein ausdifferenzierter formularmäßiger Hinweis genügen, ${ }^{356}$ denn eine (quasi) rechtliche Beratung des Schuldners ist nicht

354 LG Göttingen, ZinsO 2003, 815 (815f.); AG Köln, NZI 2005, 171 (171); HK/Eickmann, § 175 Rn.13, FK/Kießner, § 175 Rn.9; MüKo-InsO/Nowak, § 175 Rn.14.

355 Rechtsausschuss zum RegE, BT-Drucks. 14/6468, S.17f.; Kehe/Meyer/Schmerbach, ZInsO 2002, 660 (661); Kübler/Prütting/Bork/Pape, § 175 Rn.7f. m.w.N.

356 FK/Ahrens, § 302 Rn.10d. 
geschuldet. ${ }^{357}$ Dieser Hinweis ist Ausdruck der besonderen Fürsorge gegenüber rechtlich wenig informierten Schuldnern, für die das Insolvenzverfahren und die anschließende Restschuldbefreiung existenzielle Bedeutung haben. Daher kann dem Schuldner erforderlichenfalls ein Rechtsanwalt zur Prüfung, ob ein Widerspruch zweckmäßig ist oder nicht, beigeordnet werden. ${ }^{358}$

Wann dieser Hinweis zu erfolgen hat, ist im Gesetz nicht ausdrücklich geregelt. Nach Sinn und Zweck des $\S 175$ II InsO hat dies so rechtzeitig zu erfolgen, dass dem Schuldner ausreichend Zeit verbleibt zu prüfen, ob er einen Widerspruch einlegen möchte oder nicht. Der Hinweis des Insolvenzgerichts erfolgt am zweckdienlichsten unmittelbar nach Niederlegung der Tabelle, mit der Ladung des Schuldners zum mündlichen Termin oder der Bestimmung der Widerspruchsfrist im schriftlichen Prüfungsverfahren. ${ }^{359}$

Somit bleibt festzuhalten, dass die Regelung des $\S 175$ II InsO dem Insolvenzgericht einen gewissen zeitlichen Spielraum hinsichtlich der Vornahme seiner Hinweispflicht gegenüber dem Schuldner eröffnet. Dies erweist sich als vorteilhaft im Zusammenhang mit einer flexiblen Planung und Durchführung von eventuell notwendigen weiteren Prüfungsterminen oder schriftlichen Prüfungsverfahren im späteren Verfahrensstadium. Denn unterbleibt eine (hinreichende) Belehrung, ist sie zu kurzfristig oder erfolgt sie nicht in der erforderlichen Form, wird dem Schuldner gem. § 186 I InsO die Wiedereinsetzung in den vorigen Stand zu gewähren sein, ${ }^{360}$ was durch eine sorgfältige, aber eben flexible Planungsmöglichkeit des Insolvenzgerichts vermieden werden kann. Insofern kann die Hinweispflicht des Insolvenzgerichts, aufgrund ihres mittelbaren Zusammenhangs mit der Planung und der Durchführung des Verfahrens, nicht auf den Insolvenzverwalter übertragen werden. ${ }^{361}$ Gleichwohl wird in der Praxis auch der Insolvenzverwalter den Schuldner frühzeitig auf das

BGH NZI 2004, 39 (40).

BGH NZI 2004, 39 (39); LG Trier NZI 2006, 243 (244); Braun/Specovius, § 175 Rn.26; HK/Irschlinger, § 175 Rn.14.

359 Braun/Specovius, § 175 Rn.27; Graf-Schlicker/Graf-Schlicker, § 175 Rn.14.

360 Für den Wiedereinsetzungsantrag des Schuldners gilt die Ausschlussfrist des § 324 III ZPO, so dass der Antrag innerhalb eines Jahres nach Beendigung des Prüfungstermins beim Insolvenzgericht angebracht werden muss. - FK/Ahrens, $\S 302$ Rn.10d; Kübler/Prütting/Bork/Pape, § 186 Rn.4 m.w.N.

361 Siehe auch unter E.II.4. (S.198ff.). 
Vorliegen von Forderungsanmeldungen aus vorsätzlich begangener unerlaubter Handlung hinweisen.

Fraglich im Rahmen des $\S 175$ II InsO ist jedoch, ob man aus $\S 175$ II InsO nicht auch eine allgemeine Hinweispflicht des Insolvenzgerichts gegenüber den anmeldenden Insolvenzgläubigern auf die Rechtsfolge des § 302 Nr.1 InsO nur in umgekehrter Weise ableiten könnte, also dahingehend, dass Forderungen aus vorsätzlich begangener unerlaubten Handlung ohne Angabe dieses Forderungsattributs in der Anmeldung von der Restschuldbefreiung erfasst werden. Dieser (negative) Hinweis wird einem (positiven) Hinweis, dass die Forderung nicht ausgeschlossen ist, wenn das Forderungsattribut angemeldet wird, vorzuziehen sein. Denn wie der Schuldner sollte auch der Insolvenzgläubiger ausschließlich auf negative (Rechts-)Folgen eines Tuns oder Unterlassens hingewiesen werden. Dieser allgemeine Hinweis könnte im Rahmen der Bekanntmachung des Eröffnungsbeschlusses und der Aufforderung zur Anmeldung erfolgen ${ }^{362}$ und wäre mit keinem zusätzlichen Aufwand verbunden.

Die Hinweispflicht des $\S 175$ II InsO wurde jedoch ausdrücklich im Hinblick auf den rechtsunkundigen Schuldner eingeführt, dessen aktive Mitwirkung am Verfahren sich in der Praxis - außer in der (seltenen) Eigenverwaltung - durch die umfassenden Aufgaben und Tätigkeiten des Insolvenzverwalters und der geringen eigenen Einwirkungsmöglichkeit ${ }^{363}$ auf das Insolvenzverfahren regelmäßig nur auf bloße Auskunftserteilungen beschränkt. Insofern setzt sich der Schuldner im Gegensatz zu den übrigen Verfahrensbeteiligten wenig bis gar nicht mit dem allgemeinen Ablauf und den Folgen des Insolvenzverfahrens auseinander. Die Hinweispflicht hat insbesondere auch deswegen zu erfolgen, da gegen das Forderungsattribut nur der Schuldner selbst und gerade nicht, wie in allen anderen Fällen, der Insolvenzverwalter „für den Schuldner“ widersprechen kann. ${ }^{364}$

Im Gegensatz zum Schuldner sind die anmeldenden Insolvenzgläubiger aktiv und umfassend in das Verfahren durch Stimm- und umfassendere Widerspruchsrechte (feststellungshindernd) eingebunden und setzen sich insofern mit dem Verfahren und seinen Folgen wesentlich intensiver

362 Siehe bereits unter D.III. (S.33ff.).

363 Der Widerspruch des Schuldners hat keine feststellungshindernde Wirkung. Siehe dazu E.I.4.c) (S.144ff.).

364 Siehe ausführlich unter E.I.4.c)cc) (S.148ff.). 
auseinander. Weiterhin wird der Insolvenzgläubiger im Rahmen der Bekanntmachung des Eröffnungsbeschlusses frühzeitig auf einen Antrag des Schuldners auf Restschuldbefreiung hingewiesen. Darüber hinaus ist der anmeldende Insolvenzgläubiger durch $\S 174$ II InsO über die Möglichkeit der Angabe des Forderungsattributs in der Anmeldung nicht nur informiert, sondern wird dem Wortlaut nach sogar dazu angehalten - bei einem (möglichen) Vorliegen dieses Forderungsgrundes -, diesen genauso wie den Grund und den Betrag der Forderung in jedem Fall in der Anmeldung mit anzugeben. Auf $\S 174$ InsO wird im Rahmen des Eröffnungsbeschlusses und der Aufforderung zur Anmeldung auch stets verwiesen (§ 28 I 1 InsO). Hält sich der Insolvenzgläubiger bei seiner Anmeldung an den ohnehin gesetzlich vorgegebenen Rahmen, kann er bei angegebenem Forderungsattribut mit seiner Insolvenzforderung aus der privilegierten Rechtsfolge des $\S 302 \mathrm{Nr}$.1 InsO nicht herausfallen und braucht daher auf die negativen Folgen einer fehlenden Angabe des Forderungsattributs auch nicht hingewiesen zu werden. Insofern sind die Insolvenzgläubiger bei Befolgung der bestehenden Hinweise hinreichend gegen ein Herausfallen aus dem Privileg des § 302 Nr.1. InsO geschützt. Genau hiergegen war der Schuldner bisher nicht hinreichend gewarnt gewesen, weshalb sich der Gesetzgeber zu Recht veranlasst sah, eine entsprechende Hinweis- und Aufklärungspflicht gegenüber dem Schuldner in § $175 \mathrm{II}$ InsO zu normieren.

Daher bleibt festzuhalten, dass keine planwidrige Regelungslücke im Rahmen des § 175 II InsO hinsichtlich einer allgemeinen Hinweispflicht auf die Folgen des $\S 302$ Nr.1 InsO auch gegenüber den anmeldenden Insolvenzgläubigern seitens des Insolvenzgerichts besteht. 


\section{Wirkungen der Anmeldung}

Im Folgenden soll erörtert werden, welche Verfahrensrechte dem Insolvenzgläubiger durch die Anmeldung seiner Insolvenzforderung eröffnet werden und wie diese miteinander korrespondieren. Dabei wird der Frage nachzugehen sein, ob im Einzelfall zur Begründung einzelner Verfahrensrechte Ausnahmen zur Anmeldung möglich bzw. zuzulassen sind, damit bestimmte Insolvenzgläubigerinteressen im Prüfungs- und Feststellungsverfahren (überhaupt) gewahrt werden können. Darüber hinaus soll aufgezeigt werden, welchen Einfluss die übrigen Verfahrensbeteiligten im Allgemeinen, das Insolvenzgericht im Besonderen und der Schuldner im Ausnahmefall (Eigenverwaltung) auch selbst auf die Wahrnehmung der Verfahrensrechte des Anmeldenden haben können.

\section{Verfahrensbeteiligung und Widerspruchsrecht}

Durch die ordnungsgemäße Anmeldung seiner Forderung wird der Insolvenzgläubiger formell Verfahrensteilnehmer ${ }^{365}$ des Insolvenzverfahrens, wodurch er wie alle übrigen formellen Verfahrensbeteiligten fortan den Regelungen der $\S \S 174 \mathrm{ff}$. InsO unterfällt. Er erwirbt dadurch gem. § 176 InsO das Recht, im Prüfungstermin die Forderungen anderer Gläubiger $\mathrm{zu}$ bestreiten ${ }^{366}$ und selbst entsprechende Widersprüche durch Klageerhebung im Rahmen der Feststellungsklage gem. §§ 179ff. InsO zu beseitigen. ${ }^{367}$ Ein Widerspruchsrecht kann nämlich nur denjenigen Insolvenzgläubigern zugesprochen werden, deren Forderungen und Interessen untereinander unmittelbar Einfluss und Bedeutung für den Verlauf und das Ergebnis des Feststellungsverfahrens haben, das seinen Ausdruck in der Quote und dem Verteilungsverzeichnis gem. § 188 InsO findet. Die gegenseitige Prüfung und Anerkennung oder eben das

\footnotetext{
Siehe zur Differenzierung bereits unter C.I. (S.10ff.).

366 MüKo-InsONowak, § 176 Rn.27; HHK/Preß/Henningsmeier, § 176 Rn.8; BK/Breutigam, § 176 Rn.8; KS/Eckardt, S.757 Rn.27; Smid/Smid, § 176 Rn.13; Kübler/Prütting/Bork/Pape, § 176 Rn.10; Hess, InsO, § 176, Rn.16; Gottwald/Eickmann, § 64 Rn.3.

367 Eine Rechtshängigkeit i.S.d. § 261 ZPO lediglich durch Anmeldung der Forderung erfolgt daher nicht. - Baumbach/Lauterbach/Albers/Hartmann, ZPO (2009), Rn.8.
} 
Bestreiten kann neben dem Insolvenzverwalter ( $§ 179$ I InsO) nur denjenigen zustehen und von denjenigen abhängig sein, die auch tatsächlich gegenseitig Insolvenzforderungen zu prüfen und anzuerkennen haben, indem ihre Forderungen nämlich ordnungsgemäß angemeldet wurden. ${ }^{368}$ Meldet daher ein Insolvenzgläubiger seine Forderung nicht an, so wird er auch nicht formell Verfahrensteilnehmer und es kann ihm auch kein Widerspruchsrecht zustehen. Hiervon $\mathrm{zu}$ unterscheiden ist die allgemeine Teilnahmeberechtigung am Prüfungstermin und sonstigen Gläubigerversammlungen und das dadurch gewährte rechtliche Gehör, ${ }^{369}$ das grundsätzlich „allen Insolvenzgläubigern“ (§ 74 I 2 InsO) und damit auch den nachrangigen Insolvenzgläubigern (Umkehrschluss aus § 77 I 2 InsO) $)^{370}$ aufgrund ihrer materiellrechtlichen Insolvenzgläubigerstellung als Verfahrensbetroffene und ihrer damit verbundenen Einbeziehung in die Verfahrensfolgen zusteht. ${ }^{371}$

Das Gesetz hat dabei nicht geregelt, ob und unter welchen Voraussetzungen auch nachrangige Insolvenzgläubiger zur Tabelle angemeldete Insolvenzforderungen bestreiten können. Kommen sie von vornherein nicht als widerspruchsberechtigt in Betracht, weil ihre nachrangigen Insolvenzforderungen - wie dies in der Regel der Fall sein wird $^{372}$ - nicht angemeldet werden können, oder muss bei ihnen auf die Voraussetzung für das Widerspruchsrecht, dass es sich um eine zulässige und ordnungsgemäß angemeldete Insolvenzforderung handeln muss, verzichtet werden?

Teilweise wird darauf hingewiesen, dass aus dem Stimmrechtsausschluss des § 77 I 2 InsO für nachrangige Insolvenzgläubiger nicht gleichzeitig geschlossen werden könne, dass nachrangige Insolvenzgläubiger auch kein Widerspruchsrecht besitzen, zumal sie zur Teilnahme (,alle Insolvenzgläubiger“, § 74 I 2 InsO) am Prüfungstermin berechtigt sind und

368 Siehe auch die Ausführungen zur unterschiedlichen zu beurteilenden Einsichtnahme der Akten gem. § 299 I und II ZPO unter D.VI.3. (S.108ff.).

369 Näher dazu bei KS/Vallender, S.249ff.

370 Vgl. Begr. Allgemeiner Teil des RegE, BT-Drucks. 12/2443, S.99; Uhlenbruck/Uhlenbruck, § 74 Rn.7; MüKo-InsO/Ehricke, § 74 Rn.27; näher Pape, ZinsO 1999, 305 (308) m.w.N.

371 Begr. RegE InsO, BT-Drucks. 12/2443, Teil A, S.99; siehe ferner MüKoInsO/Ganter, § 1 Rn.58, Ehricke, § 74 Rn.27; Kübler/Prütting/Bork/Pape, § 176 Rn.11; Graf-Schlicker/Graf-Schlicker, § 176 Rn.10; Uhlenbruck/Uhlenbruck, § 74 Rn.10 jeweils m.w.N.

372 Dazu bereits unter C.III. (S.20ff.). 
auch der § 176 S.2 InsO lediglich von dem „Insolvenzgläubiger“ und damit auch von den nachrangigen Insolvenzgläubigern als widerspruchsberechtigt ausgehen würde. Daher würde nachrangigen Insolvenzgläubigern auch ohne eine vorherige Anmeldung ihrer Forderungen ein generelles Widerspruchsrecht im Prüfungstermin zustehen. $^{373}$

Diese Meinung differenziert jedoch nicht hinreichend zwischen der bloßen Teilnahmeberechtigung am Prüfungstermin als Gläubigerversammlung (§ 74 I 2 InsO) und dem sich aus den Vorschriften und der Systematik des speziellen Anmelde- und Feststellungsverfahrens der $\S \S 174 \mathrm{ff}$. InsO separat ergebenden Widerspruchsrecht. Im Gegensatz zu § 74 I 2 InsO im allgemeinen Teil der Insolvenzordnung, der ausdrücklich von „alle Insolvenzgläubiger“ als Verfahrensbeteiligte (jedoch im materiellen Sinn, §§ 38, 39 InsO) ${ }^{374}$ spricht, kann im Rahmen von § 176 I 2 InsO hinsichtlich des Wortlautes „Insolvenzgläubiger“, dessen Bezug sich durch seine Stellung innerhalb der Regelungen der $\S \S 174 \mathrm{ff}$. InsO lediglich auf das Anmelde- und Feststellungsverfahren bezieht, ${ }^{375}$ nur von solchen Insolvenzgläubigern ausgegangen werden, die formell Verfahrensbeteiligte durch eine ordnungsgemäße Anmeldung ihrer Insolvenzforderungen geworden sind. Zudem wird nicht berücksichtigt, dass der Gesetzgeber jegliche (formelle) Verfahrensbeteiligung der nachrangigen Insolvenzgläubiger wegen des meist fehlenden wirtschaftlichen Wertes ihrer Forderungen als unnötige Belastung und daher als Ausnahme angesehen hat. ${ }^{376}$ Dass für nachrangige Insolvenzgläubiger, soweit keine abweichenden Regelungen normiert sind, dieselben Regelungen wie für die Insolvenzgläubiger gelten sollen, ${ }^{377}$ kann hinsichtlich der Erlangung des Widerspruchsrechts als formeller Verfahrensbeteiligter natürlich nur dahingehend auszulegen sein, dass genau wie die nicht-nachrangigen Insolvenzgläubiger auch die nachrangigen Insolvenzgläubiger hierfür ihre Insolvenzforderung anzumelden haben. Auch wenn aus § 77 I 2 InsO und

\footnotetext{
373 Kübler/Prütting/Bork/Pape, § 176 Rn.11; Nerlich/Römermann/Becker, § 176 Rn.16; Uhlenbruck/Uhlenbruck, § 176 Rn.10; MüKo-InsO/Schumacher, § 178 Rn.21; GrafSchlicker/Graf-Schlicker, § 176 Rn.10.

374 Siehe auch unter E.I.2. (S.134f.).

Siehe bereits unter C.I. (S.10ff.).

376 Siehe bereits unter C.III. (S.20ff.).

377 Begr. zu $\S 201$ (§ 174 InsO) RegE, BT-Drucks. 12/2443, S.184. Dazu bereits unter C.III. (20ff.).
} 
der Versagung eines Stimmrechts vielleicht nicht unmittelbar auch ein Widerspruchsverbot der nachrangigen Insolvenzgläubiger herzuleiten sein sollte, so kann diese Normierung aber im Umkehrschluss, da auch $\S 77$ InsO generell an die Anmeldung der Insolvenzforderung und damit an die formelle Insolvenzgläubigerstellung anknüpft, als Bestätigung dafür herangezogen werden, dass die nachrangigen Insolvenzgläubiger den nicht-nachrangigen Insolvenzgläubigern (zumindest) bezüglich einer notwendigen Anmeldung gleichzustellen sind. Aufgrund dessen, dass in § 176 InsO hinsichtlich des Widerspruchsrechts gerade keine ausdrücklich abweichende Regelung wie in § 77 I 2 InsO normiert ist, kann daher auch den nachrangigen Insolvenzgläubigern nur dann ein Widerrufsrecht zustehen, wenn auch sie ihre Forderungen ordnungsgemäß angemeldet haben. Insofern ergibt sich aus § 77 I 2 InsO zumindest mittelbar - wenn auch nicht generell, mangels entsprechend zu fordernder Anmeldung aber regelmäßig - ein Widerspruchsverbot. Nur dieses Ergebnis steht auch im Einklang mit dem Verfahrensgrundsatz, dass nur derjenige (nachrangige) Insolvenzgläubiger auf die Insolvenzforderungen der übrigen Insolvenzgläubiger Einfluss haben kann, der sich selbst der Einflussmöglichkeit auf die eigene (nachrangige) Insolvenzforderung stellt. Diese gegenseitige Einflussnahme wäre nämlich umgekehrt, also bei der Annahme eines generellen Widerspruchsrechts für nachrangige Insolvenzgläubiger, den übrigen am Verfahren teilnehmenden Insolvenzgläubigern aufgrund der regelmäßig nicht anzumeldenden nachrangigen Insolvenzforderungen verwehrt.

Dass die nachrangigen Insolvenzgläubiger - wenn überhaupt - erst nachträglich zur Anmeldung ihrer Forderungen aufgefordert werden und sie daher die Forderungen anderer Insolvenzgläubiger wegen des bereits erfolgten Prüfungstermins nicht mehr bestreiten könnten, wird grundsätzlich keinen gravierenden Nachteil darstellen, weil alle Verfahrensteilnehmer die gleichen Interessen im Feststellungsverfahren verfolgen und zweifelhaften Forderungen daher bereits von einem der 
übrigen Beteiligten - insbesondere vom Insolvenzverwalter widersprochen wäre. ${ }^{378}$

Folglich haben nachrangige Insolvenzgläubiger regelmäßig kein Widerspruchsrecht. Auch sie werden ausschließlich durch ihre ordnungsgemäße Forderungsanmeldung, wenn auch erst nach gerichtlicher Aufforderung zur Anmeldung ihrer Forderungen (§ 174 III InsO), wie jeder andere Insolvenzgläubiger, formell Verfahrensbeteiligte und damit widerspruchsberechtigt. ${ }^{379}$

\section{Stimmrecht}

Das Stimmrecht ermöglicht dem Insolvenzgläubiger die Mitbestimmung über die Art und Weise der Durchführung und des Fortgangs des Insolvenzverfahrens im Rahmen der Beschlüsse der Gläubigerversammlungen. Das Stimmrecht ist damit - zusammen mit dem zuvor besprochenen Widerspruchsrecht - Ausdruck bzw. elementarer Bestandteil der Selbstverwaltungskompetenz der Insolvenzgläubiger/ -versammlung im Insolvenzverfahren.

Im Folgenden soll die Abhängigkeit des Stimmrechts von der (gegenseitigen) Ausübung des Widerspruchsrechts erörtert und dabei aufgezeigte Probleme einem interessensgerechten Lösungskonzept zugeführt werden.

378 FK/Kießner, § 176 Rn.8 und 14 will den nachrangigen Insolvenzgläubigern ein grundsätzliches Widerspruchsrecht einräumen, das aber nur dann Wirkung entfalten soll, wenn die nachrangigen Insolvenzgläubiger bis zur Erstellung des Schlussverzeichnisses zur Anmeldung ihrer Forderungen aufgefordert wurden. Ansonsten blieben diese Widersprüche unberücksichtigt. Solch eine Regelung würde zu unerträglichen Schwebezuständen hinsichtlich der Erhebung von Feststellungsklagen führen. Zudem findet sie in keiner Hinsicht eine gesetzliche Stütze und ist daher abzulehnen.

379 HK/Irschlinger, § 176 Rn.2; Hess, InsO, § 176 Rn.26; Gottwald/Eickmann, § 63 Rn.3; KS/Eckhardt, S.757 Rn.27; Smid/Smid, § 178 Rn.3; BK/Breutigam, § 176 Rn.8; HHK/Preß/Henningsmeier, § 176 Rn.4, § 178 Rn.8. 


\section{a) Stimmrechtsgewährung und Einigung über das Stimmrecht}

Die wirksam angemeldete und weder vom Insolvenzverwalter noch von einem stimmberechtigten Gläubiger bestrittene Insolvenzforderung ${ }^{380}$ gewährt dem Insolvenzgläubiger ein Stimmrecht in der Gläubigerversammlung (§ $77 \quad$ I 1 , II $\quad$ InsO). ${ }^{381}$ Doch auch die Insolvenzgläubiger der im Prüfungstermin oder im schriftlichen Verfahren bestrittenen Forderungen sind stimmberechtigt, soweit in der Gläubigerversammlung eine Einigung über das Stimmrecht erfolgt oder bei Uneinigkeit das Insolvenzgericht dem Gläubiger ein Stimmrecht zubilligt (§ 77 II 1, 2 InsO). Das Insolvenzgericht kann seine Entscheidung auf Antrag des Insolvenzverwalters oder eines Insolvenzgläubigers abändern (§ 77 II 3 InsO). ${ }^{382}$ Ohne diese weitergehenden Regelungen des Stimmrechts würde aus der Möglichkeit des Bestreitens folgen, dass der betroffene Insolvenzgläubiger bis zum rechtskräftigen Abschluss der von ihm nach $\S \S 179 \mathrm{ff}$. InsO zu betreibenden Feststellungsklage von der Mitwirkung in den Gläubigerorganen ausgeschlossen wäre. ${ }^{383}$ Damit wäre

380 Ausschließlich an die persönliche Insolvenzforderung gegenüber dem Schuldner (§ 38 InsO) knüpft die Anmeldung gem. § 174 InsO und damit auch die allgemeine Stimmrechtsfeststellung gem. § 77 InsO an, so dass $\S 77$ InsO auf absonderungsberechtigte Forderungen, die keine persönlichen Forderungen sind, grundsätzlich keine Anwendung findet und damit auch kein allgemeines Stimmrecht besteht. In $\S 77$ III InsO wurden lediglich entsprechend klarstellend die vormaligen Regelungen des § $96 \mathrm{KO}$ und § 71 III VerglO übernommen, die ebenfalls ausschließlich die persönliche Forderung gegen den Schuldner zum Gegenstand hatten. Vgl. Begr. zu $\S 88$ (§ 77 InsO) RegE, BT-Drucks. 12/2443, S.134. Hiervon klar abzugrenzen ist daher die Ausnahmeregelung des § 76 II 2. Halbsatz InsO, die im Beschlussfall der Gläubigerversammlung ausdrücklich auch die absonderungsberechtigten Gläubiger, denen der Schuldner nicht persönlich haftet hinsichtlich eines nach § 77 InsO festzulegenden Stimmrechts im Rahmen ihres Ausfalls erfasst. Hiervon ebenfalls abzugrenzen sind die umfassenden Sonder-regelungen im Rahmen der Abstimmung eines Insolvenzplans (§§ 241ff. InsO), wonach wohl selbst nachrangigen Insolvenzgläubigern ein Stimmrecht zustehen kann. Diese weiterführende Thematik soll jedoch vom Umfang dieser Arbeit ausgeschlossen sein.

381 Auf die Ausübung des Stimmrechts bei Abstimmungen im Insolvenzverfahren soll im Rahmen dieser Arbeit nicht weiter eingegangen werden. Siehe dazu ausführlich etwa bei Oelrichs, Gläubigermitwirkung.

382 Die materielle Berechtigung des Gläubigers bleibt von jedweder Entscheidung über das Stimmrecht unberührt. Auch auf seine Antrags- und Beschwerderechte (§§ 78, 216, 251, 253, 289 InsO) hat die Entscheidung über das Stimmrecht keinen Einfluss. Vgl. Begr. zu § 88 (§ 77 InsO) RegE, BT-Drucks. 12/2443, S.133f.; MükoInsO/Ehricke, § 77 Rn.34 m.w.N.

383 In der Praxis würde das bei der Dauer eines Insolvenzfeststellungsverfahrens häufig dazu führen, dass einzelne Insolvenzgläubiger von der Mitwirkung vollkommen ausgeschlossen blieben. 
es ein Leichtes, durch Erhebung eines Widerspruchs - (rechts-) missbräuchlich $^{384}$ - andere Insolvenzgläubiger grundsätzlich von der Mitwirkung im Verfahren bis zur Klärung im Feststellungsprozess auszuschalten. $^{385}$ Dieser Missbrauchsgefahr treten die abgestuften Regelungen des $\S 77$ II 1 und 2 InsO entgegen. Die Einigung zwischen dem Insolvenzverwalter und allen erschienenen stimmberechtigten Insolvenzgläubigern über das Stimmrecht einer bestrittenen Insolvenzgläubigerforderung hat aufgrund der Gläubigerautonomie im Verfahren grundsätzlich Vorrang vor einer gerichtlichen Entscheidung (§ 77 II 2 InsO). Die Einigung schließt dabei den Inhaber der bestrittenen Forderung mit ein, ${ }^{386}$ wodurch auch im Rahmen der Einigung die Gefahr eines rechtsmissbräuchlichen Stimmrechtsausschlusses seitens der übrigen Verfahrensteilnehmer weitgehend ausgeschlossen wird. Da die Höhe der Insolvenzforderung entscheidend bei der Stimmrechtsausübung ist (vgl. $\S 76$ II InsO), muss es den Abstimmungsberechtigten auch möglich sein, zu entscheiden, dass sie nicht mit dem vom Inhaber behaupteten - daher bestrittenen - Wert, sondern nur zu einem Bruchteil desselben für die Stimmabgabe zu bewerten ist. Dies hat zur Folge, dass die Stimme des zugelassenen Insolvenzgläubigers ein geringeres Gewicht hat. Bezüglich der Differenz zum vollen Wert der Insolvenzforderung kann der Anmeldende dann wiederum die Entscheidung des Insolvenzgerichts beantragen. ${ }^{387}$

Haben stimmberechtigte Insolvenzgläubiger wechselseitig jeweils die Forderung des anderen bestritten, so ist dem Wortlaut nach weder die Zuordnung der betroffenen Forderungen zu § 77 I InsO noch zu Absatz II möglich, denn es kann hier wie dort nicht entschieden werden, ob ein Bestreiten im Sinne des § 77 InsO (eines „stimmberechtigten Gläubigers“)

384 Die Materialien zur Konkursordnung sprachen insoweit von „frivolen oder böswilligen Motiven“. Siehe KO-Motive, S.314 = Hahn, KO, S.287.

385 FK/Kind, § 77 Rn.7; MüKo-InsO/Ehricke, § 77 Rn.9; Oelrichs, Gläubigermitwirkung, S.73f.; näher Pape, ZIP 1991, 837 (839f.).

386 Nach dem Willen des Gesetzgebers sollte die Regelung des § $95 \mathrm{KO}$ und $\S 71$ II VerglO in $\S 77$ InsO übernommen werden, wonach eine Einigung des Forderungsanmeldenden und des der Forderung Widersprechenden nötig war. - Begr. RegE zu $\S 88$ (§ 77 InsO), BT-Drucks. 12/2443, S.133; AG Hamburg ZinsO 2005, 1002 (1002f.); Smid/Smid, § 77 Rn.5; Braun/Herzig, § 77 Rn.8; näher auch MüKoInsO/Ehricke, § 77 Rn.10.

387 MüKo-InsO/Ehricke, § 77 Rn.11; Braun/Herzig, § 77 Rn.9. 
überhaupt vorliegt. ${ }^{388}$ Damit eine gegenseitige Blockade vermieden wird, sind solche Forderungen § 77 II InsO zuzuordnen, ${ }^{389}$ um eine Entscheidung im Konsens zu finden. Dadurch wird sichergestellt, dass eine Entscheidung zumindest über ein vorläufiges Stimmrecht möglich ist, die Gläubigerversammlung auf einer breiten Abstimmungsbasis handlungsfähig bleibt und damit der Fortgang des Insolvenzverfahrens nicht behindert wird. Die Feststellung der Insolvenzforderung zur Tabelle (§§ 178 I 1, 183 I, II InsO) sichert das Stimmrecht sodann endgültig. ${ }^{390}$ Einer differenzierten Betrachtung bedarf es bei „vorläufig“ bestrittenen Forderungen des Insolvenzverwalters, soweit sie nicht auch durch einen Insolvenzgläubiger bestritten worden ist. Das „vorläufige Bestreiten“ durch den Insolvenzverwalter bringt zum Ausdruck, dass er - etwa aus Zeitgründen in besonders großen Verfahren - zur endgültigen Prüfung bisher außer Stande war und deshalb gerade noch offen ist, ob er die Forderung anerkennen will oder „endgültig“ bestreitet. Soweit man diese Möglichkeit des Bestreitens bejaht und deren Auswirkung für den Anmeldenden im weiteren Verfahrens berücksichtigt, ${ }^{391}$ dürfen dem Gläubiger dadurch nicht auch noch Nachteile hinsichtlich seines Stimmrechts entstehen. ${ }^{392}$ Daher sollte den Gläubigern „vorläufig“ bestrittener Insolvenzforderungen - unabhängig davon, ob bereits eine Prüfung der Insolvenzforderung stattgefunden hat oder nicht ${ }^{393}$ - bis $\mathrm{zu}$ deren „endgültiger“ Bestreitung zunächst ein volles Stimmrecht unabhängig von § 77 II InsO zustehen. ${ }^{394}$

Anmeldende nachrangige Insolvenzgläubiger (§ 77 I 2 InsO) sowie Insolvenzgläubiger, die ihre Forderungen nicht angemeldet oder zurückgezogen haben, haben hingegen kein Stimmrecht (mehr) in der

388 Siehe auch unter E.I.1. (S.128ff.).

389 MüKo-InsO/Ehricke, § 77Rn.8; HK/Eickmann, § 77 Rn.4; Jaeger/Gerhardt, InsO, $\S 77$ Rn.8.

390 Zum Stimmrecht vor Ablauf der Anmeldefrist und ungeprüfter Forderungen siehe unter E.I.1. (S.128ff.).

391 Dazu unter E.I.4.d)bb) (S.160ff.)

392 BGH NZI 2005, 31 (31); LG Göttingen ZIP 1989, 1471 (1471);

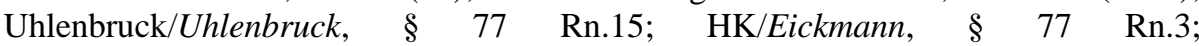
Kübler/Prütting/Bork/Kübler, § 77 Rn.19; Pape, ZIP 1991, 837 (844).

393 Grundsätzlich zu bejahen, wenn über die Feststellung „vorläufig“ bestrittener Forderungen noch nicht entschieden worden ist und der Forderung im Termin nicht widersprochen wird. - BGH NZI 2005, 31 (31). Siehe auch ab E.I.1. (S.128ff.).

394 So auch Uhlenbruck/Uhlenbruck, § 77 Rn.15; Pape, ZIP 1991, 837 (844); differenzierend MüKo-InsO/Ehricke, § 77 Rn.17. 
Gläubigerversammlung. ${ }^{395}$ Ihre Widersprüche haben daher keine Auswirkungen auf das Stimmrecht anderer Insolvenzgläubiger. Gleichwohl können sie zu Mitgliedern des vorläufigen Gläubigerausschusses ( $§ 67$ III InsO) bestellt werden, ebenso wie sie in der Gläubigerversammlung in einen Gläubigerausschuss gewählt werden können (§ 68 II InsO). ${ }^{396}$ Ohne Stimmrecht sind weiterhin die Massegläubiger nach $\S 53$ InsO, da ihre Ansprüche regelmäßig erst nach Einleitung des Verfahrens entstehen (vgl. §§ 54, 55) und gesondert zu befriedigen sind, sowie aussonderungsberechtigte Gläubiger, die gem. $\S 47$ Satz 1 InsO gerade keine Insolvenzgläubiger sind und damit auch nicht aus der Insolvenzmasse befriedigt werden. ${ }^{397}$

\section{b) Stimmrecht bei Widerspruch des Schuldners in Eigenverwaltung}

Der Widerspruch des Schuldners hat im normalen Insolvenzverfahren keine feststellungshindernde Wirkung (vgl. § 178 I 2 InsO), ${ }^{398}$ weil ihm mit Eröffnung des Insolvenzverfahrens gem. § 80 I InsO die Verwaltungs- und Verfügungsbefugnis über sein Vermögen, das zur Insolvenzmasse gehört, entzogen und dem Insolvenzverwalter übertragen wird. Dem Schuldner steht deshalb auch kein Stimmrecht zu, so dass sein Widerspruch keinen Einfluss auf das Stimmrecht der formellen Insolvenzgläubiger hat. Fraglich ist jedoch, ob dem feststellungshindernden Widerspruch des Schuldners in Eigenverwaltung eine stimmrechtsbeschränkende Wirkung zukommt. In der Literatur wird dies, soweit ersichtlich, regelmäßig verneint. ${ }^{399}$ Zwar erlaube § 283 I 2 InsO dem Schuldner, im Rahmen seiner Eigenverwaltung mit feststellungshindernder Wirkung eine Insolvenzforderung zu bestreiten, doch hätte dies keine Bedeutung für die Stimmberechtigung des betreffenden Insolvenzgläubigers. Denn zum einen erwähne § 77 I InsO das Bestreiten des Schuldners als Einschränkung der

395 HK/Irschlinger, $\S 174$ Rn.9; Kübler/Prütting/Bork/Pape, $\S 174$ Rn.36; Uhlenbruck/Uhlenbruck, § 174 Rn.28.

396 Innerhalb des Gläubigerausschusses bestimmt sich das Stimmrecht jedoch nach Köpfen und nicht nach der Höhe eventueller Insolvenzforderungsbeträge, so dass jedem Mitglied nur eine Stimme zusteht. Dieser Unterschied ist deshalb geboten, weil auch Nichtgläubiger Ausschussmitglieder werden können.

399 Uhlenbruck/Uhlenbruck, § 77 Rn.9; MüKo-InsO/Ehricke, § 77 Rn.7 m.w.N. 
Stimmrechtsgewährung weder direkt noch über $\S 283$ I 2 InsO. Zum anderen ist der Schuldner bei der Eigenverwaltung weder selbst stimmberechtigt, so dass er auch kein Recht haben könne, bei den Einigungsverhandlungen der Versammlung dadurch Einfluss zu nehmen, die Versammlung oder das Insolvenzgericht zu einer Stimmrechtsfestsetzung zu zwingen, noch trete er an die Stelle des Insolvenzverwalters. An dessen Stelle tritt vielmehr der Sachwalter (§ 270 II 1 InsO), der somit die Möglichkeit des Bestreitens hat und aufgrund seiner Stellung mit seinem Bestreiten auch das Stimmrecht des Insolvenzgläubigers blockieren kann.

Die Literatur übersieht in ihrer Argumentation jedoch, dass der Schuldner in der Eigenverwaltung hinsichtlich der Prüfung der Insolvenzforderungen und auch des feststellungshindernden Widerspruchs den Insolvenzgläubigern und dem Sachwalter durch § 283 I InsO ausdrücklich, gerade weil nicht weiter differenziert wird, gleichgestellt ist. ${ }^{400} \S 270$ I 2 InsO bestimmt nämlich insoweit, dass, soweit gerade keine Abweichungen für die Eigenverwaltung bestimmt worden sind, die allgemeinen Vorschriften für das Verfahren gelten. Hinsichtlich der Gleichstellung des Eigenverwalters bedeutet dies aber, gerade weil keine weiteren Regelungen in $\S 77$ InsO bzw. § 283 InsO bezüglich der Eigenverwaltung des Schuldners vorgenommen worden sind, dass an den feststellungshindernden Widerspruch des Eigenverwalters die gleichen Einwirkungsmöglichkeiten auf das Verfahren gebunden sein müssen, wie bei den übrigen Widerspruchsberechtigten. Zudem kennt die Insolvenzordnung nur einheitliche Rechtsfolgen (vgl. §§ 77 I, II; 178 I, III; 201 II InsO) für den feststellungshindernden ( $\S 178$ I 1 InsO) und vollstreckungshindernden (§ 178 I 2 InsO) Widerspruch, so dass auch eine, wie von der Literatur behauptet, aufgespaltete Wirkungsweise - beim Eigenverwalter zwar feststellungshindernd, aber ohne Auswirkung auf das Stimmrecht - keine Stütze in der Insolvenzordnung finden kann. Wenn dem so wäre, würde der zugesprochene eigene feststellungshindernde Widerspruch des Eigenverwalters neben dem des Sachwalters außerdem ausgehöhlt, zweckbefreit und damit nahezu sinnlos werden. Es ist nämlich nicht ersichtlich, warum dem Insolvenzgläubiger durch eine aufgespaltete bzw.

400 Begr. zu § 344 (§ 283 InsO) RegE, BT-Drucks. 12/2443, S.226. 
beschränkte Wirkung des Eigenverwalterwiderspruchs ein Vorteil für sein Stimmrecht entstehen sollte. Wenn eine ordnungsgemäß angemeldete Forderung materiellrechtlich nicht oder teilweise nicht zur Teilnahme am (weiteren) Insolvenzverfahren berechtigt und ihr daher zu widersprechen ist, so kann dem anmeldenden Gläubiger hinsichtlich dieser Forderung grundsätzlich - unabhängig von § 77 II InsO - auch kein entsprechendes Stimmrecht eingeräumt werden (§ 77 I 1 InsO). Auch wenn der Sachwalter grundsätzlich gehalten ist, unberechtigten Forderungen selbst zu widersprechen, wird der Regelungsinhalt des § 283 I InsO dennoch ad absurdum geführt, wenn dem Sachwalter und den Insolvenzgläubigern neben dem Eigenverwalterwiderspruch stets ein eigener Widerspruch gegen die unberechtigte Forderung abzufordern wäre, um letztlich die „volle Widerspruchswirkung“ auch im Rahmen des § 77 I 1 InsO erreichen zu können.

Dass der Schuldner grundsätzlich kein Stimmrecht hat, wird zur Begründung ebenfalls nicht angeführt werden können; der Insolvenzverwalter/ Sachwalter/ Treuhänder hat trotz Widerspruchsrechts nämlich ebenfalls kein Stimmrecht. Zwar beruht dessen Widerspruchsrecht auf seiner neutralen Amtsstellung und Pflicht zur Wahrung aller Verfahrens- und Beteiligteninteressen, doch ist es in der Eigenverwaltung gerade Sinn und Zweck, dass der Schuldner aus positiver Eigeninitiative und Selbstinteresse das Verfahren in erster Linie „selbst in die Hand nimmt““. ${ }^{401}$ Damit dies gleichwohl ordnungsgemäß und nicht missbräuchlich geschieht, wurde dem Eigenverwalter der Sachwalter an die Seite gestellt. Stellt der Sachwalter Umstände fest, die erwarten lassen, dass die Fortsetzung der Eigenverwaltung zu Nachteilen für die Gläubiger führen wird, so hat er dies gem. § 274 III InsO unverzüglich dem Gläubigerausschuss und dem Insolvenzgericht anzuzeigen, so dass schließlich jederzeit eine Aufhebung der Anordnung der Eigenverwaltung beantragt werden kann (vgl. § 272 InsO). Insofern wird man für eine aufgespaltete Wirkungsweise auch nur äußerst eingeschränkt mit einer Missbrauchsmöglichkeit des Widerspruchs durch den Eigenverwalter argumentieren können. Zuletzt stellt der Gesetzgeber in seiner Begründung

401 Das ohnehin seltene Verfahren in Eigenverwaltung wird nämlich regelmäßig auf Sanierung des eigenen Unternehmens und selten auf Liquidation gerichtet sein. Siehe auch Begr. zur Eigenverwaltung RegE, BT-Drucks.12/2443, S.223. 
zu $\quad \S 283$ InsO ausdrücklich selbst klar, dass ein Widerspruch des Schuldners in Eigenverwaltung die gleiche Auswirkung auf das Stimmrecht hat, wie der des Sachwalters oder widerspruchsberechtigten Insolvenzgläubigers. ${ }^{402}$

Folglich sind mit dem feststellungshindernden Widerspruch des Schuldners in Eigenverwaltung (§ 283 I 2 InsO) die gleichen Wirkungsund Einwirkungsmöglichkeiten auf das Insolvenzverfahren verbunden wie mit den Widersprüchen des Sachwalters und den übrigen Insolvenzgläubigern. Hinsichtlich einer Einigung gem. § 77 II InsO ist jedoch zum Schutz der Interessen sämtlicher Verfahrensteilnehmer der Sachwalter zuständig. ${ }^{403}$

\section{c) Stimmrechtsfestsetzung durch das Insolvenzgericht}

Die insolvenzgerichtliche Stimmrechtsentscheidung ist gegenüber der Stimmrechtseinigung aufgrund der im Insolvenzverfahren herrschenden Gläubigerautonomie subsidiär, so dass im Falle einer Einigung dem Insolvenzgericht eine Entscheidung über das Stimmrecht oder dessen Neufestsetzung untersagt ist. ${ }^{404}$ Bedarf es mangels Einigung dennoch einer Entscheidung durch das Insolvenzgericht (§ 77 II 2 InsO), so liegt diese grundsätzlich im pflichtgemäßen Ermessen des verfahrensleitenden Insolvenzgerichts, ob dem betreffenden Gläubiger überhaupt ein Stimmrecht zusteht und gegebenenfalls welchen Umfang dieses Recht aufweist. Dadurch sollen die Funktions-/ Beschlussfähigkeit der Gläubigerversammlung und der zügige Fortgang des Verfahrens sichergestellt werden. Die Entscheidung des Insolvenzgerichts erfolgt aufgrund einer kursorischen Prüfung der Anmeldung und der Forderung („Forderungsfeststellung“), wobei alle vorgetragenen und vorliegenden (Beweis-) Faktoren des anmeldenden Gläubigers und des Widersprechenden, die für und gegen das Bestehen der angemeldeten

402 In § 283 InsO wurden nämlich sinngemäß die Regelungen der §§ 71 I, II; 85 I VglO übernommen. Begr. zu § 344 (§ 283 InsO) RegE, BT-Drucks. 12/2443, S.226. Vgl. auch Begr. zu § 344 (§ 283 InsO) des Rechtsausschusses, BT-Drucks.12/7302, S.122 und S.186.

403 Smid/Smid, § 77 Rn.9.

404 BGH ZIP 2004, 2339 (2341); AG Duisburg NZI 2003, 447 (447); Küber/Prütting/Kübler, § 77 Rn.17; MüKo-InsO/Ehricke, § 77, Rn.10ff. m.w.N. 
Forderung und deren Höhe als Insolvenzforderung sprechen, abzuwägen sind. ${ }^{405}$ Das Insolvenzgericht hat dabei den jeweiligen Vortrag auf seine sachliche Glaubhaftigkeit und seine rechtliche Schlüssigkeit hin zu prüfen. ${ }^{406}$ Die gerichtliche Stimmrechtsentscheidung soll einerseits gewährleisten, dass ein Gläubiger mit bestrittener Forderung nicht völlig von der Verfahrensteilnahme ausgeschlossen wird, wenn die Forderung nicht unzweifelhaft ist. Andererseits soll verhindert werden, dass nicht abstimmungsberechtigte Gläubiger die Entscheidungen der Gläubigerversammlung dominieren oder entscheidend beeinflussen. ${ }^{407}$ Bestehen aufgrund ausgeglichener Vorträge offene Zweifel, sollte das Stimmrecht grundsätzlich gewährt werden, um eine möglichst breite Abstimmungsbasis in den Versammlungen zu sichern (,in dubio pro creditore“). ${ }^{408}$ Insofern findet zwar eine kursorische Prüfung der angemeldeten Forderung durch das Insolvenzgericht statt, jedoch hat diese aufgrund der ermessensfreien (bloßen) Stimmrechtsfeststellung durch das Insolvenzgericht keinerlei Einfluss auf den eigentlichen materiellrechtlichen Bestand der angemeldeten Forderung oder deren Feststellung im Sinne des $\S 178$ III InsO. ${ }^{409}$ Dies wäre auch - wie bereits festgestellt ${ }^{410}$ - mit der im Anmelde- und Feststellungsverfahren der §§ 174ff. InsO generell fehlenden „eigenständigen Entscheidungsmöglichkeit“ des Insolvenzgerichts hinsichtlich der materiellrechtlichen Zulässigkeit der angemeldeten Insolvenzforderung nicht vereinbar. Die Entscheidung des Insolvenzgerichts über die Stimmrechtsfeststellung ergeht dabei durch Beschluss und ist grundsätzlich zu begründen, ${ }^{411}$ wobei die Stimmrechtsentscheidung des Insolvenzgerichts unanfechtbar ist (§ 6 I InsO, § 11 III 2 RpflG).

$\mathrm{Zu}$ beachten ist aber die diesbezüglich durch Art. 14 EGInsO eingeführte Anfechtungsvorschrift des $\S 18$ III 2 RpflG, wonach der

\footnotetext{
Einzelheiten siehe bei MüKo-InsO/Ehricke, § 77 Rn.15ff.

HK/Eickmann, § 77 Rn.8; MüKo-InsO/Ehricke, § 77 Rn.14.

Uhlenbruck/Uhlenbruck, § 77 Rn.18.

408 So auch HK/Eickmann, § 77 Rn.8; Uhlenbruck/Uhlenbruck, § 77 Rn.18; Kübler/Prütting/Bork/Kübler, § 77 Rn.20; MüKo-InsO/Ehricke, § 77 Rn.14 m.w.N. 
Insolvenzrichter ${ }^{412}$, aufgrund eines vom formellen Insolvenzgläubiger oder Insolvenzverwalter bis zum Schluss des Abstimmungstermins gestellten Antrags, ein vom Rechtspfleger ${ }^{413}$ festgesetztes Stimmrecht neu festsetzen und die Wiederholung der Abstimmung anordnen kann, wenn sich die Stimmrechtsentscheidung des Rechtspflegers auf ein Abstimmungsergebnis ausgewirkt hat. ${ }^{414}$ Unabhängig davon besteht gem. § 77 II 3 InsO die grundsätzliche Abänderungsmöglichkeit der Stimmrechtsfeststellung des Gerichts durch einen formlosen, aber zu begründenden Antrag des Insolvenzverwalters oder eines in der Gläubigerversammlung erschienenen formellen Insolvenzgläubigers. Dieser Antrag ist begründet, wenn sich die der ersten Stimmrechtsentscheidung zugrunde gelegten Umstände oder zuvor verborgen gebliebenen Erkenntnisse geändert haben. ${ }^{415}$ Der Abänderungsantrag gem. § 77 II 3 InsO kann jedoch im Gegensatz zum Antrag gem. § 18 III RpflG, der ausschließlich Bezug auf die Entscheidung des Rechtspflegers nimmt, unabhängig davon gestellt werden, ob der Rechtspfleger oder der Insolvenzrichter die Stimmrechtsentscheidung getroffen hat. Er kann allerdings nur vor einer Abstimmung gestellt werden, im Rahmen derer es auf die (erneute) Stimmrechtsfeststellung ankommt. Nach der Abstimmung bleibt allein der Weg der Anfechtung der Stimmrechtsfeststellung nach § 18 III 2 RpflG. Dies belegt der Wortlaut des $§ 18$ III 2 RpflG („Hat sich die Entscheidung des Rechtspflegers auf das Abstimmungsergebnis ausgewirkt“) und ergibt sich zudem aus der dort - und nicht auch in § 77 II InsO - angeordneten Wiederholung der Abstimmung. Gleichwohl ist auch die Abänderungsentscheidung des Rechtspflegers nach § 77 II 3 InsO gem. § 18 III 2 RpflG anfechtbar, da es sich weiterhin um „eine Entscheidung des Rechtspflegers“ handelt, die als solche einer (weiteren) Fremdüberprüfung des Insolvenzrichters unterworfen werden kann. Etwas anderes gilt hingegen dann, wenn der

412 Sollte der zuständige Insolvenzrichter nicht kurzfristig zur Verfügung stehen, wird die Gläubigerversammlung zu vertagen sein (§ 74 II 2 InsO). Siehe auch bei KS/Bernsen, S.1853f. Rn.29.

413 Dies wird gem. § 3 Nr.2e RpflG regelmäßig der Fall sein.

414 Mit dieser Neuerung will der Gesetzgeber die gegen die Unanfechtbarkeit der Stimmrechtsentscheidung erhobenen verfassungsrechtlichen Bedenken ausräumen. Begr. zu Art.14 Nr.5 RegE EGInsO, BT-Drucks. 12/3803, S.65. Siehe zu weiteren Einzelheiten ausführlich bei MüKo-InsO/Ehricke, § 77 Rn.25ff.

415 Kübler/Prütting/Bork/Kübler, § 77 Rn.24; MüKo-InsO/Ehricke, § 77 Rn.21f. m.w.N. Von Amts wegen findet daher grundsätzlich keine Überprüfung der eigenen Entscheidung statt. 
Insolvenzrichter zuvor abgeändert hat, denn dann liegt keine Rechtspflegerentscheidung mehr vor und es bleibt bei einer Unanfechtbarkeit (§ 6 InsO). ${ }^{416}$

Zusammengefasst bedeutet dies, dass eine für ein Abstimmungsergebnis der Gläubigerversammlung ausschlaggebende Stimmrechtsentscheidung des Rechtspflegers in jedem Fall einer rechtsbehelfsmäßigen Überprüfung durch den Insolvenzrichter zugänglich ist (§ 18 III RpflG). Dadurch wird für die Insolvenzgläubiger nicht nur die Stimmrechtsentscheidung an sich, sondern indirekt auch der durch sie ermöglichte Beschluss anfechtbar. Durch die gleichzeitige Neuabstimmung trägt § 18 III RpflG darüber hinaus zur Verfahrensbeschleunigung und -vereinfachung bei, indem nicht erst auf das Aufhebungsverfahren gem. § 78 I InsO zurückgegriffen werden muss.

\section{Akteneinsicht und Erteilung von Abschriften/Kopien}

Wie bereits im Rahmen des Niederlegungsorts der Tabelle angedeutet wurde, ${ }^{417}$ haben insbesondere zu Beginn des Insolvenzverfahrens die Verfahrensbeteiligten oder sogar am Insolvenzverfahren nicht unmittelbar beteiligte Dritte ein gesteigertes Interesse daran, an verfahrensinterne Informationen $\mathrm{zu}$ gelangen. ${ }^{418}$ Dieses Informationsinteresse reicht von legitimen wirtschaftlichen Interessen bis hin zum Interesse, interne Informationen lediglich als Druckmittel gegen den Schuldner einsetzen zu können. Offenkundig ist, dass von der Publikation interner Informationen über die wirtschaftliche Verfassung des Schuldners Gefahren für dessen Ruf ausgehen, die sich in empfindlicher Weise nachteilig auf seine künftige wirtschaftliche Entwicklung auswirken können. Dies wird besonders dadurch deutlich, dass die verfahrensrechtlichen, zwangsweise durchsetzbaren Auskunftspflichten des Insolvenzschuldners (§§ 5, 20, $97 f$. InsO) weit über diejenigen hinausgehen, die ihm im gewöhnlichen

416 Wie hier MüKo-InsO/Ehricke, § 77 Rn. 21, 25, wohl auch HK/Eickmann, § 77 Rn.12f.; a.A. Kübler/Prütting/Bork/Kübler, § 77 Rn.24.

417 Siehe bereits unter D.V.3. (S.87ff.).

418 Die Insolvenzordnung sieht vielfach vor, dass die gerichtlichen Insolvenzakten durch Verfahrenbeteiligte eingesehen werden können. Vgl. z.B. §§ 66 II 2, 150, 154, 175, 188 S.2, 234 InsO. 
Geschäfts- und Rechtsverkehr obliegen (vgl. etwa § 807 ZPO). Zur Kenntnis des Insolvenzverwalters, der Gläubigerorgane oder des Insolvenzgerichts können damit höchst sensible, persönliche Daten über den Schuldner gelangen, an deren Geheimhaltung er schutzwürdige Interessen hat. Insofern setzt sein aus dem Persönlichkeitsrecht fließendes Recht auf informationelle Selbstbestimmung ${ }^{419}$ nicht nur den Verfahrensbeteiligten, sondern insbesondere dem Informationsverlangen von unbeteiligten Dritten Grenzen. ${ }^{420}$ Diesem Spannungsverhältnis muss daher die theoretische wie die konkrete praktische Entscheidung auch über das Recht zur Akteneinsicht Rechnung tragen.

Die Konkursordnung enthielt keine besonderen Vorschriften über die Zulässigkeit der Einsicht in die Insolvenzakten. Infolge dessen stützte man allgemein das Recht zur Akteneinsicht auf eine entsprechende Anwendung des $\S 299$ ZPO i.V.m. $\S 72$ KO. ${ }^{421}$ Demgegenüber hatte die Vergleichsordnung, das im Verhältnis zur Konkursordnung neuere Gesetz, die Akteneinsicht eigenständig in § 120 VglO geregelt. Solch eine Regelung hat in die Insolvenzordnung jedoch keine vergleichbare Aufnahme gefunden, so dass die zur Konkursordnung entwickelten Grundsätze zur Akteneinsicht des § 299 ZPO nunmehr über $\S 4$ InsO entsprechend auf die Insolvenzordnung zu übertragen sind. ${ }^{422}$

Demnach sind gem. § 299 I ZPO nach Eröffnung des Insolvenzverfahrens als „Parteien“ im Sinne dieser Vorschrift alle „Verfahrensbeteiligten“ einsichtsberechtigt. ${ }^{423}$ Verfahrensbeteiligte sind im eröffneten Insolvenzverfahren der Insolvenzverwalter, der Insolvenzschuldner, alle Insolvenzgläubiger, die Mitglieder des Gläubigerausschusses und die absonderungsberechtigten Gläubiger, die eine persönliche Forderung

419 Siehe dazu das Volkszählungsurteil des BVerfG in BVerfGE 65, 1ff.

420 Näher Jaeger/Gerhardt, InsO, § 4 Rn.18 m.w.N.

421 Siehe nur HK/Kirchhof, § 4 Rn.13; Uhlenbruck/Uhlenbruck, § 4 Rn.25ff. jeweils m.w.N. Ausführlich Heil, Akteneinsicht und Auskunft im Konkurs, Rn.161ff.

422 OLG Frankfurt 2005, 1327 (1328); OLG Celle ZIP 2004, 370 (371); OLG Celle ZIP 2004, 368 (368); Kübler/Prütting/Bork/Pape, § 20 Rn.17; Uhlenbruck/Uhlenbruck, § 4 Rn.25ff.; MüKo-InsO/Ganter, § 4 Rn.57; HK/Kirchhof, § 4 Rn.13; Heeseler, ZInsO 2001, 873 (882f.) m.w.N.

423 Eine Versagung oder Beschränkung kann nach allgemeinen Grundsätzen nur bei offensichtlichem Rechtsmissbrauch in Frage kommen. - Pape, ZIP 2004, 598 (599f.); Uhlenbuck/Uhlenbruck, § 175 Rn. 12; Gottwald/Eickmann, § 63 Rn.38; MüKoInsO/Nowak, § 174 Rn.12 m.w.N. 
gegen den Schuldner angemeldet haben (vgl. § 74 I 2 InsO). ${ }^{424}$ Hinsichtlich der Insolvenzgläubiger werden zwar alle mit Eröffnung des Insolvenzverfahrens - materiellrechtlich durch § 38 InsO erfasst - kraft Gesetz in das Verfahren und dessen Folgen einbezogen, doch formell Verfahrensteilnehmer und damit „Partei“ im Sinne des Zivilprozesses werden sämtliche Insolvenzgläubiger ( $\S \S 38,39$ InsO) im Gegensatz zu den übrigen Verfahrensteilnehmern erst mit der Anmeldung ${ }^{425}$ ihrer Forderungen gem. §§ 174ff. InsO. ${ }^{426}$ Dies ist auch nur sachgerecht und folgerichtig, da der Insolvenzverwalter und auch die Mitglieder des Gläubigerausschusses - zumindest jene Mitglieder (vgl. § 67 III InsO), die nicht sowieso schon durch eine bereits erfolgte Anmeldung formell Verfahrensbeteiligte sind - schon bzw. einzig aufgrund ihrer wohlbedachten Bestellung seitens des Insolvenzgerichts (§§ 58 I; 67 I InsO) oder durch Wahl der stimmberechtigten Mitglieder der Gläubigerversammlung ( $\S \S 57 ; 68$ InsO) und damit kraft ihres Amtes formell Verfahrensbeteiligte werden. ${ }^{427}$ Insofern kommt bei ihnen zeit ihres Amtes eine Stellung als außenstehende „Dritte“ im Sinne des § 299 II InsO - im Gegensatz zu am Verfahren teilnehmenden und nicht teilnehmenden Insolvenzgläubigern - schon gar nicht in Betracht.

Als „Partei“ im Sinne des § 299 I ZPO und damit als grundsätzlich einsichtsberechtigt sind daher ausschließlich alle formellen Verfahrensbeteiligten des Insolvenzverfahrens anzusehen und dies solange, bis sie ihre formelle Verfahrensstellung verlieren. ${ }^{428}$ Diese Einordnung ermöglicht eine eindeutige Differenzierungsmöglichkeit zu § 299 II ZPO, wonach der nicht am Verfahren teilnehmende „Dritte“ für die Einsichtnahme stets sein rechtliches Interesse glaubhaft machen muss (vgl. $\S 294$ ZPO). Ein anerkennenswertes rechtliches Interesse ist in der Regel

424 MüKo-InsO/Nowak, § 175 Rn.12; Graf-Schlicker/Graf-Schlicker, § 175 Rn.11; Nerlich/Römermann/Becker, § 175 Rn.7; Jaeger/Gerhardt, InsO, § 4 Rn.21; HK/Kirchhof, \& 4 Rn.13 m.w.N.

425 Siehe schon unter C.I. (S.10ff.). Gleiches wird hinsichtlich der absonderungsberechtigten Gläubiger zu gelten haben, denen der Schuldner zwar nicht persönlich haftet, die aber gleichwohl ihre Absonderungsrechte anzumelden haben und hinsichtlich § 76 II 2. Halbsatz InsO Verfahrensbeteiligte sind. - Vgl. Begr. zu § 85 (§ 74 InsO) RegE, BT-Drucks. 12/2443, S.133.

426 OLG Frankfurt 2005, 1327 (1328); OLG Celle ZIP 2004, 370 (371); MüKoInsO/Ganter, § 4 Rn.61; HK/Kirchhof, § 4 Rn.15, Braun/Bußhardt, § 4 Rn.30 m.w.N. Dazu auch bereits unter D.VI.1. (S.94ff.).

427 Dass der Insolvenzschuldner formell Verfahrensbeteiligter ist, ergibt sich kraft Natur der Sache.

428 Siehe dazu unter E.I.4.e) (S.163ff.). 
gegeben, wenn Belange des Antragstellers konkret berührt sind, die einen rechtlichen oder wirtschaftlichen Bezug ${ }^{429}$ zum Inhalt der einzusehenden Akten haben. ${ }^{430}$ Insbesondere hier entsteht das bereits angesprochene Spannungsverhältnis im Insolvenzverfahren zwischen dem Recht des Schuldners auf informationelle Selbstbestimmung und dem Informationsbedürfnis des am Verfahren (bisher) unbeteiligten Dritten. Daher hat bei einem Einsichtsbegehren das entscheidungsbefugte Organ grundsätzlich eine ermessensfehlerfreie Abwägung und Entscheidung im Einzelfall zwischen dem anerkennenswerten rechtlichen Informationsinteresse des antragenden Dritten und den berechtigten Geheimhaltungsinteressen des Insolvenzschuldners zu treffen. ${ }^{431}$ In Bezug zur Anmeldung werden regelmäßig diejenigen Insolvenzgläubiger ein rechtliches Interesse i.S.d. § 299 II ZPO glaubhaft machen können, die zunächst durch Einsicht in die Unterlagen prüfen wollen, ob sich eine Anmeldung ihrer Forderungen zum Verfahren überhaupt lohnt. Je tiefer die begehrten Informationen in die Persönlichkeitssphäre des Schuldners eindringen, desto höher liegen jedoch die Anforderungen, die an das glaubhaft zu machende rechtliche Interesse des Einsichtsbegehrenden zu stellen sind. ${ }^{432}$ Dem Insolvenzschuldner ist dabei vor einer positiven Entscheidung zur Einsichtnahme eine Gelegenheit zu geben, unter Vorbehalt des Möglichen und Zumutbaren, sein Geheimhaltungsinteresse geltend $\mathrm{zu}$ machen. ${ }^{433}$ Daher bedeutet das generelle bzw. erteilte Recht zur Einsichtnahme in die Akten gem. § 299 ZPO im Fall des Insolvenzverfahrens nicht immer gleichzeitig auch das Recht auf unbegrenzte Einsichtnahme. § 299 ZPO ist generell nur entsprechend und unter Berücksichtigung der Besonderheiten des Insolvenzverfahrens anwendbar. ${ }^{434}$ Daher können Teile von Akten ${ }^{435}$

429 Hierzu ausführlich BGH ZIP 2006, 1154 (1156).

430 OLG Frankfurt ZInsO 2005, 1327 (1328); OLG Brandenburg NZI 2002, 49 (49); Zöller/Greger, ZPO § 299 Rn.6a; Haarmeyer/Seibt, Rpfleger 1996, 221 (226); MüKo-InsO/Ganter, § 4 Rn.62 m.w.N.

431 Siehe Einzelheiten bei MüKo-InsO/Ganter, § 4 Rn.75.

432 Jaeger/Gerhardt, InsO, § 4 Rn.24 m.w.N.

433 BGH ZIP 1998, 961 (962); OLG Köln NZI 1999, 502 (502); Zöller/Greger, ZPO § 299 Rn.6-6b; Thomas/Putzo/Reichold, ZPO, § 299 Rn.3; MüKo-InsO/Ganter, § 4 Rn.70; Jaeger/Gerhardt, InsO, $\S 4 \quad$ Rn.32; HK/Kirchhof, $\S 4 \quad 4 \quad$ Rn.13; Uhlenbruck/Uhlenbruck, § 4 Rn.32 m.w.N.

434 Siehe bereits unter D.I. (S.23ff.).

435 Insbesondere Schriftstücke, die sich auf Vorgänge beziehen, deren Geheimhaltung das Insolvenzgericht im Interesse des Zwecks des Insolvenzverfahrens für erforderlich hält, wie z.B. Protokolle des Gläubigerausschusses, Gläubigertabellen, Berichte über Bezugs- und Absatzmöglichkeiten, Patente, Lizenzen, Erfindungen etc. 
- über § 299 IV ZPO hinaus - grundsätzlich von der Einsicht durch Dritte, ${ }^{436}$ im Einzelfall aber auch für Verfahrensteilnehmer ausgeschlossen werden, wenn insoweit ein besonderes Geheimhaltungsinteresse besteht (vgl. vormals § $120 \mathrm{II}$ VglO) ${ }^{437}{ }^{438}$ Dies wäre - neben dem besonderen Geheimhaltungsinteresse des Schuldners - etwa dann der Fall, wenn durch eine unbeschränkte Akteneinsicht der Verfahrenszweck (z.B. Sanierung eines Unternehmens, § 1 S.1 2.Alt InsO) gefährdet würde ${ }^{439}$ oder die Gefahr eines Missbrauchs offensichtlich ist. Teile der Akten können jedoch nur Personen verwehrt werden, die nicht ohnehin zu einer Geheimhaltung ihrer amtswegig erlangten Informationen verpflichtet sind. Hierzu zählt der Insolvenzgläubiger als auch entsprechende Mitglieder des Gläubigerausschusses, ${ }^{440}$ denen somit grundsätzlich ein uneingeschränktes Einsichtrecht zusteht. ${ }^{441}$

Die Einsichtnahme findet während des Verfahrens ausschließlich auf der Geschäftsstelle des Insolvenzgerichts statt, da angesichts einer großen Zahl einsichtberechtigter Personen die Akten jederzeit verfügbar sein müssen. $\mathrm{Zu}$ eben diesem Zweck ist die Tabelle dort mit allen andern Unterlagen vom Insolvenzverwalter niederzulegen (§ 175 I 2 InsO). Ein Einsichtsrecht beim Insolvenzverwalter vor der Niederlegung besteht damit nicht.

Neben der Akteneinsicht gewährt § 299 I ZPO zudem die Möglichkeit, sich auf eigene Kosten durch die Geschäftsstelle Ausfertigungen, Auszüge und Abschriften der Tabelle und der Akten erteilen zu lassen. Eingeschränkt werden kann der Anspruch auf Erteilung von Kopien und Abschriften allenfalls dann, wenn durch das Gesuch die personellen und sachlichen Möglichkeiten des Insolvenzgerichts überfordert sind und dies

436 Siehe auch Pape, ZIP 2004, 598 (602).

437 § 120 II VglO: „Gläubigern kann die Einsicht in solche Teile der Akten versagt werden, deren Kenntnis für sie ohne Bedeutung ist oder deren Geheimhaltung nach Angabe des Schuldners für die Fortführung seines Unternehmens erforderlich ist.“ - Näher Pape, ZIP 1997, 1367 (1368).

438 OLG Celle ZInsO 2004, 204 (205); LG Darmstadt ZIP 1990, 1425 (1425); AG Dresden ZInsO 2002, 147 (147f.); FK/Schmerbach, § 4 Rn.63; MüKo-InsO/Ganter, $\S 4$ Rn.75; Braun/Bußhardt, § 4 Rn.36; Jaeger/Gerhardt, InsO, § 4 Rn.29; HHK/Rüther, § 4 Rn.41; HK/Kirchhof, § 4 Rn.13 m.w.N.

439 BGH ZIP 1983, 344 (344); LG Darmstadt ZIP 1990, 1424 (1424f.); Jaeger/Gerhardt, InsO, § 4 Rn.29 m.w.N.

440 Zur gesetzlichen Schweigepflicht der Mitglieder des Gläubigerausschlusses siehe etwa bei Jaeger/Gerhardt, InsO, § 69 Rn.10 m.w.N.

441 Letzteres unterliegt lediglich den Einschränkungen einer Interessenskollision etwa hinsichtlich einer gleichzeitigen Beschäftigung bei einem konkurrierenden Unternehmen. - Jaeger/Gerhardt, InsO, § 4 Rn.29 m.w.N. 
entsprechend dem Gesuchstellenden mitgeteilt und begründet wird. ${ }^{442}$ Fraglich ist dabei jedoch, ob und inwieweit auch Dritten die Möglichkeit von Kopien und Abschriften zu ermöglichen ist, da § 299 II ZPO diese Möglichkeit nicht explizit vorsieht. Im Rahmen des Insolvenzverfahrens wäre die ausschließliche Möglichkeit zur Informationsbeschaffung im Fall des § 299 II ZPO durch die Einsichtnahme auf der Geschäftsstelle zu eng. Dabei würde nicht berücksichtigt werden, dass der grundsätzlich gegebene Anspruch auf Akteneinsicht, gerade in Insolvenzsachen, bei denen Dritte bzw. Insolvenzgläubiger, die vor ihrer Forderungsanmeldung zunächst ihre Erfolgsaussichten durch Akteneinsicht prüfen wollen und ihren Sitz häufig sehr weit vom Insolvenzgericht entfernt haben, dadurch unterlaufen werden kann, dass zwar formal Einsicht in die Insolvenzakten bewilligt wird, tatsächlich aber die Einsicht dadurch unmöglich gemacht wird, dass die Einsichtnehmenden finanzielle Aufwendungen durch die Einsicht haben, die außer Verhältnis zu dem Nutzen einer solchen Einsicht stehen. Würde hier nicht die Möglichkeit von Kopien und Abschriften gewährt werden, würde diese Entscheidung letztlich auf eine Rechtsversagung hinauslaufen, weil gerade ein wirtschaftlich denkender Insolvenzgläubiger verständlicherweise nicht bereit sein wird, erhebliche Fahrt- und Personalkosten aufzuwenden, um Akteneinsicht zu nehmen. Verglichen mit den Kosten der Anfertigung von Kopien aus den Akten wird man in einem solchen Fall davon auszugehen haben, dass das Insolvenzgericht - trotz fehlenden Wortlauts - im Rahmen des § 299 II ZPO und seiner freien Ermessenentscheidung ob und auf welche Art und Weise Akteneinsicht zu gewähren ist, im Einzelfall auch die Möglichkeiten der Versendung von Kopien und Abschriften berücksichtigen muss. ${ }^{443}$ Darüber hinaus gewährt § 299 III 1 ZPO auch die Möglichkeit der Einsichtnahme durch die Übermittlung von elektronischen Dokumenten etwa im Rahmen einer E-Mail, was eine kostengünstige Alternative darstellt. Dies insbesondere im Hinblick darauf, dass die Insolvenztabelle dem

442 OLG Celle ZInsO 2004, 204 (204); LG Karlsruhe ZInsO 2004, 690 (690); Pape, ZIP 1997, 1367 (1368); ders., ZIP 2004, 598 (604); HHK/Rüther, § 4 Rn.43; MüKoInsO/Ganter § 4 Rn.72 m.w.N.

443 OLG Celle ZInsO 2007, 150 (151f.); ZIP 2004, 368 (369f.); 370 (371); OLG Hamm ZIP 2004, 283 (283); HK/Kirchhof, § 4 Rn.15; MüKo-InsO/Ganter, § 4 Rn.72; Braun/Bußhardt, § 4 Rn.31; HHK/Rüther, § 4 Rn.49; Uhlenbruck/Uhlenbruck, § 4 Rn.35. 
Insolvenzgericht heutzutage ohnehin meist in elektronischer Form vorliegt und daher zumindest die Tabelle unproblematisch und kostengünstig per Internet verschickt werden kann. In diesem Rahme wäre in konkret abgewogenen Einzelfällen und bei bestehender Möglichkeit auch das einscannen von weiteren Unterlagen denkbar, wobei in jedem Fall § 299 III 4 ZPO zu beachten ist, der die Übermittlung mit einer qualifizierte elektronische Signatur vorschreibt. Insoweit muss das Insolvenzgericht bei den vom Gesuchsteller vorgebrachten Gründen konkret abwägen, ob es zumutbar ist, ihn ausschließlich auf eine Einsicht an Ort und Stelle zu verweisen.

Zuständig für die Gewährung der Akteneinsicht gem. § 299 I ZPO ist regelmäßig der Urkundsbeamte der Geschäftsstelle oder der Rechtspfleger. Im Fall des § 299 II ZPO ist der Vorstand des Gerichts (Präsident oder Direktor des Amtsgerichts) zuständig, der die Entscheidungsbefugnis aber auf den zuständigen Richter delegieren kann, wovon verbreitet Gebrauch gemacht wird.

Wird einem Verfahrensbeteiligten die Akteneinsicht oder Erteilung von Kopien/Abschriften versagt oder nicht unbeschränkt gewährt, ist dagegen bei Entscheidung durch den Urkundsbeamten der Geschäftsstelle die Erinnerung (§ 573 I ZPO i.V.m. § 4 InsO), bei Entscheidung durch den Rechtspfleger die Rechtspflegererinnerung (§ 11 II 1 RPflG) und bei Entscheidungen des Richters die sofortige Beschwerde (§ 567 I Nr.2 ZPO i.V.m. § 4 InsO) eröffnet. ${ }^{444}$

Wird die Akteneinsicht im Fall des § 299 II ZPO vom Gerichtsvorstand bzw. Richter versagt, hat der Anbringende die Möglichkeit, dagegen einen Antrag auf gerichtliche Entscheidung gem. §§ 23ff. EGGVG zu stellen, da es sich insoweit um Justizverwaltungshandeln des Gerichts handelt. ${ }^{445}$ Über den Antrag entscheidet gem. § 25 I EGGVG das zuständige Oberlandesgericht.

\footnotetext{
444 Siehe nur MüKo-InsO/Ganter, § 6 Rn.57ff. m.w.N.

445 OLG Dresden ZIP 2003, 39 (39); OLG Brandenburg NZI 2002, 49 (49); HHK/Rüther, § 4 Rn.50; MüKo-InsO/Ganter, § 4 Rn.69 m.w.N.
} 


\section{Verjährungshemmung und Verjährung}

Die ordnungsgemäße Anmeldung einer Insolvenzforderung zum Insolvenzverfahren führt zudem mit Eingang beim Insolvenzverwalter gem. § 204 I Nr.10 BGB zu einer Hemmung der Verjährung in Höhe des angemeldeten Betrages. ${ }^{446}$ Der Zeitraum, während dessen die Verjährung gehemmt ist, wird in die Verjährungsfrist nicht eingerechnet, § 209 BGB.

Aufgrund dieser Wirkung muss der Insolvenzverwalter durch geeignete organisatorische Maßnahmen sicherstellen, dass alle an ihn adressierten Schriftstücke sogleich nach Eingang auf Forderungsanmeldungen überprüft, gegebenenfalls (mittels Eingangsstempel) datiert und zusammen mit den etwa beigefügten urkundlichen Beweisstücken (§ 174 I 2 InsO) aktenmäßig aufbewahrt werden. ${ }^{447}$ Gleiches gilt für eine gem. § $174 \mathrm{IV}$ InsO zugelassene Anmeldung in Form von elektronischen Dokumenten.

Die Hemmung endet (ex nunc) gem. § 204 II 1 BGB nach (1) der rechtskräftigen Entscheidung, also durch die vollstreckbare Feststellung der Forderung zur Tabelle, wodurch, soweit ein anderes nicht bestimmt ist, ${ }^{448}$ die bis zu diesem Zeitpunkt maßgebliche Verjährungsfrist durch eine dreißigjährige Verjährungsfrist gem. § 197 I Nr.5 BGB ersetzt wird (§ 201 BGB i.V.m. §§ 201 II, 215 II 2 InsO), ${ }^{449}$ oder (2) durch die sonstige Beendigung des eingeleiteten Verfahrens, etwa durch Aufhebung (§§ 200, 258 InsO) oder Einstellung (§ 207ff. InsO), ${ }^{450}$ mit der Folge, dass dem Gläubiger in jedem Fall der Rest der gehemmten Verjährungsfrist zuzüglich einer sechsmonatigen Nachfrist zur Verfügung steht. ${ }^{451}$

446 RGZ 170, 276 (278); Staudinger/Peters, § 204 Rn.97; Palandt/Heinrichs, § 204 Rn.18, 42; Prütting/Wegen/Weinreich/Kessler, § 204 Rn.17; Erman/J.SchmidtRäntsch, § 204 Rn.26, 48.

447 KS/Eckardt, S.750 Rn.12; HK/Irschlinger, § 174 Rn.17; Häsemeyer, InsO, Rn.22.11; Kübler/Prütting/Bork/Pape, § 174 Rn.35; Hess, InsO, § 174 Rn.90; MüKoInsO/Nowak, § 174 Rn.24; Bähr, InVo 1998, 205 (208); Uhlenbruck/Uhlenbruck, $\S 174$ Rn.12.

448 Hat der Anspruch regelmäßig wiederkehrende Leistungen zum Inhalt, tritt an die Stelle der dreißigjährigen die regelmäßige dreijährige Verjährungsfrist, § 197 II BGB.

449 Palandt/Heinrichs, § 197 Rn.12f., § 201 Rn.2; MüKo-BGB/Grothe, § 197 Rn.23, 32; $\S 201$ Rn.4 m.w.N.

450 Entscheidend ist die öffentliche Bekanntmachung (§ 9 I 3 InsO) und nicht schon die Beschlussfassung (vgl. § 34 III 1 InsO). - BGHZ 63, 1 (3); Palandt/Heinrichs, § 204 Rn.42; MüKo-BGB/Grothe, § 204 Rn.101 m.w.N.

451 Erman/J.Schmidt-Räntsch, § 204 Rn.26, 48; Mohrbutter/Ringstmeier/Ernestus, § 11 Rn.22; AnwkBGB/Mansel/Budzikiewicz, § 204 Rn.1; Vallender, ZInsO 2002, 110 (111). 
Fraglich ist, inwieweit sich die Hemmung hinsichtlich einer möglichen Verjährung auf ein nachfolgendes Restschuldbefreiungsverfahren erstreckt, da § 204 II 1 BGB nur allgemein von dem eingeleiteten Verfahren spricht, das Restschuldverfahren jedoch ein eigenständiges, wenn auch eng mit dem Insolvenzverfahren verbundenes Verfahren darstellt. ${ }^{452}$

Relevant ist diese Frage jedoch nur für untitulierte Insolvenzforderungen, ${ }^{453}$ denen der Schuldner widersprochen hat. ${ }^{454}$ Denn der Widerspruch des Schuldners steht, im entscheidenden Unterschied zum Widerspruch des Verwalters oder eines Insolvenzgläubigers (§§ 179 I, 180 I InsO), einer rechtskräftigen Feststellung der Forderung nicht entgegen ( $\S$ 178 I 2 InsO). Eine Titulierung und Vollstreckbarkeit der Forderung (vgl. § 201 II 1 InsO) und damit die Wirkung der dreißigjährigen Verjährungsfrist der § 197 I Nr.5 i.V.m. § 201 BGB kann der Insolvenzgläubiger im Gegensatz zu den vom Schuldner unwidersprochenen Forderungen nur dadurch erreichen, dass er den Widerspruch des Schuldners durch eine Klage oder die Aufnahme eines anhängigen Rechtsstreits nach § 184 I InsO beseitigt. Bei einem obsiegenden Urteil kann der Insolvenzgläubiger dann nach Beendigung des Insolvenzverfahrens eine vollstreckbare Ausfertigung aus der Tabelle verlangen (§ 201 II 2, 3 InsO).

Verfolgt der betroffene Insolvenzgläubiger den Widerspruch des Schuldners dagegen nicht, läuft mit Ankündigung der Restschuldbefreiung und Aufhebung des Insolvenzverfahrens (vgl. § 289 InsO) nach der sechsmonatigen Nachfrist (§ 204 II 1 BGB) die Verjährungsfrist der vom Schuldnerwiderspruch betroffenen Insolvenzforderung weiter. Dies hätte, ungeachtet der Länge der Verjährungsfrist je nach Anspruchsgrundlage der Insolvenzforderung, aufgrund der langen Wohlverhaltensphase von sechs Jahren (§ 287 II 1 InsO) zur Folge, dass die Insolvenzforderung oftmals - trotz ihrer Feststellung durch die

452 Siehe nur Kothe/Ahrens/Grote/Ahrens, § 286 Rn.18 m.w.N.

453 Bei betitelten Forderungen ist dies unproblematisch. Denn liegt für eine vom Schuldner bestrittene Forderung ein vollstreckbarer Schuldtitel oder ein Endurteil vor, so obliegt es gem. § 184 II InsO dem Schuldner, binnen einer Frist von einem Monat, die mit dem Prüfungstermin oder im schriftlichen Verfahren mit dem Bestreiten der Forderung beginnt, den Widerspruch zu verfolgen. Nach fruchtlosem Ablauf dieser Frist gilt ein Widerspruch als nicht erhoben.

454 Bei unbestrittenen und damit vollstreckbar festgestellten Insolvenzforderungen (§ 178 I, III i.V.m. § 201 II 1 InsO) tritt nach Beschluss des Restschuldbefreiungsverfahrens und der nachfolgenden Aufhebung des Insolvenzverfahrens (vgl. § 289 InsO) hingegen unproblematisch die Wirkung der neuen dreißigjährigen Verjährungsfrist der §§ 197 I Nr.5; 201 BGB ein. 
Gläubigergemeinschaft als zur Teilnahme am Insolvenzverfahren und Befriedigung aus der Insolvenzmasse berechtigt - verjähren würde.

In diesem Zusammenhang erscheint es hinsichtlich des stets ungewissen Verlaufs des Restschuldbefreiungsverfahrens fraglich, ob der Insolvenzgläubiger bei einem Antrag des Schuldners auf Erteilung der Restschuldbefreiung für den Fortlauf der Verjährungshemmung gezwungen sein muss, bereits im Insolvenzverfahren den durch § $184 \mathrm{I}$ InsO vorgegebenen Weg zu beschreiten. Denn sollte dem Schuldner später eine Restschuldbefreiung erteilt werden, wirkt diese gegen alle Insolvenzgläubiger ( $§ 301$ I InsO) und seine Insolvenzforderung wäre nicht mehr durchsetzbar. ${ }^{455}$ Die durch die lange sechsjährige Wohlverhaltensphase erzwungene Klageerhebung des Insolvenzgläubigers ${ }^{456}$ auf Feststellung hätte ihren Zweck, den Widerspruch des Schuldners zu beseitigen, um damit zum einen, die Verjährung seiner Insolvenzforderung zu vermeiden und zum anderen, nach Abschluss des Verfahrens gegen den Schuldner aus dem Tabelleneintrag vollstrecken zu können, verfehlt. Da es aber nach der Entlassung des Schuldners in die Wohlverhaltensperiode völlig ungewiss ist, ob die Forderung vorzeitig befriedigt wird oder ob der Schuldner Restschuldbefreiung erlangt, kann der Insolvenzgläubiger aufgrund der gesetzlichen bedingten Verfahrenssituation nicht gezwungen sein, präventive Verfahrensschritte zur Wahrung seiner Interessen vorzunehmen, die nicht auch zu einem letztendlichen Erfolg führen können. Um diese verfahrens- und interessenbedingte Widersprüche aufzuheben und den Insolvenzgläubiger vor einer Verjährung seines Anspruchs während „des Verfahrens“457 zu bewahren, so dass auch noch eine spätere Durchsetzung seines Feststellungsinteresses - je nach absehbarem Ausgang des Restschuldbefreiungsverfahrens - möglich ist, hat die Verjährungshemmung des $\S 204$ I Nr.10 BGB bis zur rechtskräftigen Versagung oder Erteilung der Restschuldbefreiung fortzuwirken, wenn der Insolvenzgläubiger bei einem Widerspruch des

Siehe auch unter E.III.2. (S.208ff.).

456 Eine andere Möglichkeit des Insolvenzgläubigers zur Interessenswahrung besteht aufgrund des fortdauernden Vollstreckungsverbots des § 294 I InsO auch während der Wohlverhaltensphase nicht.

457 Schutz vor Verjährung ist schließlich der eigentliche Zweck des § 204 BGB. Begr. zu § 204 RegE, BT-Drucks. 14/6040, S.112; Palandt/Heinrichs, § 204 Rn.1. 
Schuldners gegen die Feststellung seiner Insolvenzforderung von einer Klage nach § 184 I InsO absieht. ${ }^{458}$

Dies wird jedoch nicht hinsichtlich des Forderungsgrundes von Insolvenzforderungen gelten können, die allein oder zusätzlich, also neben einem vertraglichen Anspruch, ${ }^{459}$ auch auf einer vorsätzlich begangenen unerlaubten Handlung des Schuldners beruhen (sollen). Dies aus gleich zweierlei Gründen: Zum einen kann in diesem Fall der Zweck der Feststellungsklage gem. $\S 184$ I InsO, trotz eines Restschuldbefreiungsantrags eine Nachhaftung des Schuldners persönlich zu begründen, aufgrund der Ausnahme von zur Tabelle festgestellten Insolvenzforderungen aus vorsätzlich begangener unerlaubter Handlung (§ 302 Nr.1 InsO) grundsätzlich erreicht werden. Zum anderen muss dem Grundsatz Rechnung getragen werden, dass deliktisch begründete (Insolvenz-)Forderungen einer kurzen Verjährungsfrist unterliegen, damit die in Betracht kommenden Sachverhalte möglichst zeitnah geklärt werden („verdunkelnde Macht der Zeit“), ${ }^{460}$ so dass im Einzelfall zwischen den eventuell unterschiedlichen Streitgegenständen, die gerade auf unterschiedlichen Lebenssachverhalten beruhen können (vertragliche/gesetzliche und/oder deliktische Anspruchsgrundlagen; daher auch die zusätzliche Darstellung gem. § 174 II InsO), grundsätzlich differenziert werden muss. ${ }^{461}$ Diese Differenzierung macht auch Sinn hinsichtlich einer möglichen Hemmung der Verjährung der Insolvenzforderung bezüglich des Rechtsgrundes der vorsätzlich begangenen unerlaubten Handlung eben durch (zusätzliche) Anmeldung des Forderungsattributs gem. § 174 II InsO als auch hinsichtlich der diesbezüglich möglichen Erhebung der Verjährungseinrede des Schuldners und der unterschiedlichen Betreibungslasten im Rahmen der

\footnotetext{
458 So auch Uhlenbruck/Uhlenbruck, § 174 Rn.28; Braun/Specovius, § 174 Rn.37; Vallender, ZInsO 2002, 110 (112); siehe auch OLG Rostock ZVI 2005, 433 (434). Zur Differenzierung siehe auch ausführlich unter E.I.4.c)cc) (S.148ff.).

460 Dazu ausführlich Peters, KTS 2006, 127 (131f.).

461 Kahlert, ZInsO 2005, 192 (194f.); Kübler/Prütting/Bork/Pape, § 184 Rn.117.
} 
Feststellungsprozesse gem. $\S \S 179 \mathrm{ff}$. InsO. ${ }^{462}$ Letzteres wird insbesondere im Rahmen von angemeldeten titulierten Insolvenzforderungen und hinsichtlich der Mittitulierung des Forderungsattributs von Bedeutung sein, was noch gesondert zu behandeln sein wird. ${ }^{463}$ Die Hemmung der Verjährung einer Insolvenzforderung hinsichtlich des Grundes der vorsätzlich begangenen Handlung kann jedenfalls grundsätzlich nur dann erfolgen, wenn dieser (zusätzlich) zur Tabelle angemeldet wurde. Eine anhaltende Hemmung der Verjährung der angemeldeten Insolvenzforderung bei einem eventuell beantragten Restschuldbefreiungsverfahren hinsichtlich des Rechtsgrunds der vorsätzlich begangenen unerlaubten Handlung über die Beendigung des Insolvenzverfahrens hinaus ist daher aus genannten Gründe abzulehnen.

Die Verjährung von nachrangigen Forderungen, soweit diese (noch) nicht zur Tabelle anzumelden sind, ist gesetzlich nicht geregelt. Dies ist wohl dem Umstand Rechnung zu tragen, dass der Gesetzgeber die nachrangigen Forderungen zwar mit ins Insolvenzverfahren einbinden wollte, aber selbst davon ausgeht, dass eine Befriedigung in den seltensten Fällen überhaupt eintreten wird, ${ }^{464}$ zumal auch die nachrangigen Forderungen von einer späteren Restschuldbefreiung erfasst werden, ob sie nun angemeldet wurden/konnten oder nicht. ${ }^{465}$

Will man also eine Verjährungshemmung trotz fehlender Aufforderung zur Anmeldung der nachrangigen Insolvenzforderungen erreichen, so bleibt nur eine Anmeldung der Insolvenzforderung ohne Hinweis auf ihre Nachrangigkeit, ${ }^{466}$ da solch eine Anmeldung bekanntermaßen wirksam und in die Tabelle aufzunehmen ist. ${ }^{467}$ Dies erscheint wegen einer fehlenden gesetzlichen Regelung sachgerecht. ${ }^{468}$ Und da diese Möglichkeit grundsätzlich besteht, bleibt generell kein Raum dafür, dass sich der

462 Vgl. in diesem Zusammenhang auch die inzwischen unfangreichen Entscheidungen hinsichtlich der Verletzung der Pflicht zur Abführung von Gesamtsozialversicherungsbeiträgen und des Haftungsbescheids gem. § 25 I 1 SGB IV: etwa BGH ZInsO 2006, 489 (489); OLG Dresden, ZInsO 2007, 622 (622); siehe auch Kahlert, ZInsO 2005, 192ff. m.w.N.

463 Dazu ausführlich unter E.I.4.f)bb) (S.170ff.).

464 Begr. zu § 46 (§ 39 InsO) RegE, BT-Drucks. 12/2443, S.123.

465 Bereits unter C.III. (S.20ff.).

466 Hiervon scheint indirekt auch der Gesetzgeber ausgegangen zu sein. Siehe Begr. zu $\S 201$ (§ 174 InsO) RegE, BT-Drucks. 12/2443, S.184; weiter MüKo-InsO/Nowak, $\S 174$ Rn.24; Wenner/Schuster, BB 2006, 2649 (2652). Siehe schon D.IV.5. (S.53ff.).

468 Vgl. auch hierzu die bereits erwähnte Aussage des Gesetzgebers in Fn.210 (S.55). 
Gläubiger später darauf beruft, ihm sei die Hemmung der Verjährung durch seine Forderungsanmeldung wegen der fehlenden Aufforderung durch das Insolvenzgericht nicht möglich gewesen. ${ }^{469}$

469 Insofern mag es nur in absoluten Ausnahmefällen vertretbar erscheinen, dass die nachrangige Forderung des Insolvenzgläubigers gem. § 206 BGB nicht verjährt ist, wenn sich der Insolvenzgläubiger darauf beruft, dass er darauf vertraut hat, seine Forderung erst nach Aufforderung durch das Insolvenzgericht anmelden zu müssen und davon ausging, die Forderung vor einer solchen Aufforderung nicht anmelden zu müssen/können. 


\section{Rücknahme der Anmeldung}

Die Anmeldung kann nur bis zur Feststellung der Insolvenzforderung zurückgenommen werden. ${ }^{470}$ Nach der Feststellung ist eine Rücknahme wegen der Rechtskraftwirkung des $\S 178$ III InsO nicht mehr möglich. ${ }^{471}$ Der Insolvenzgläubiger kann dann lediglich auf die Geltendmachung seines Anspruchs im weiteren (Verteilungs-)Verfahren verzichten. ${ }^{472}$

Die Rücknahme ist daher ebenso wie die Anmeldung Prozesshandlung ${ }^{473}$ und geschieht durch Erklärung in der für die Anmeldung geltenden Form gegenüber dem Insolvenzverwalter, nach Niederlegung der Tabelle gegenüber dem Gericht, wobei neben einem Vermerk für die übrigen Insolvenzgläubiger in der Tabelle dem Verwalter dies abschriftlich mitzuteilen ist. ${ }^{474}$ Dem Insolvenzgläubiger steht dann jedoch kein Stimmund Widerspruchsrecht mehr zu; Einsicht in die Akten kann ihm nur noch unter den Voraussetzungen des § 299 II ZPO i.V.m. § 4 InsO gewährt werden.

Gleichbedeutend mit der Nichtteilnahme am Verfahren bewirkt auch die Rücknahme der Anmeldung keinen gleichzeitigen Verzicht auf den materiellrechtlichen Forderungsanspruch selbst, so dass der Gläubiger seine Forderung jederzeit wieder anmelden kann. ${ }^{475}$

Anders als nach $\S \S 214$ II, 212 I BGB a.F., wonach die Verjährungsunterbrechung als nicht eingetreten galt (ex tunc), tritt mit

470 Ähnlich der Klagerücknahme gem. § 269 ZPO. Dazu etwa Stein/Jonas/Roth, ZPO, § 269 Rn.29; Musielak/Foerste, ZPO, § 269 Rn.6; Zöller/Greger, ZPO, § 269 Rn.8ff.

471 Allg. Meinung: RGZ 112, 297 (299); HHK/Preß/Henningsmeier, § 174 Rn.20; Gottwald/Eickmann, $\S 63$ Rn.39; Uhlenbruck/Uhlenbruck, $\S 174$ Rn.23; Mohrbutter/Ringstmeier/Ernestus, § 11 Rn.25; Kübler/Prütting/Bork/Pape, § 174 Rn.33; MüKo-InsO/Nowak, § 174 Rn.26; Jaeger/Weber, KO, § 139 Rn.32f.; Kuhn/Uhlenbruck, KO, § 139 Rn.11 m.w.N.

Für eine Rücknahmemöglichkeit auch nach der Feststellung: FK/Kießner, § 177 Rn.35f., Graf-Schlicker/Graf-Schlicker, § 177 Rn.10, deren Argumentation aber schon mit Blick auf den eindeutigen Willen des Gesetzgebers, durch Feststellung eine eindeutige und feststehende Rechtslage (auch) innerhalb des Verfahren zu schaffen, nicht greifen kann. Denn es handelt sich hier nicht um eine auf das Verfahren beschränkende, sondern um eine sich auf die übrigen Verfahrensteilnehmer erweiternde Titulierungswirkung und dies über das Insolvenzverfahren hinaus. Siehe bereits unter D.IV.5. (S.53ff.) dort Fn. 232 (S.59); weiter unter E.III.2. (S.208ff.).

472 MüKo-InsO/Nowak, § 174 Rn.26; Hess, InsO, § 174 Rn.111; Kuhn/Uhlenbruck, KO, $\S 145$ Rn.5.

473 Dazu bereits unter D.IV.2.a) (S.36ff.).

474 Gottwald/Eickmann, § 63 Rn.40, 42; MüKo-InsO/Nowak, § 174 Rn.26.

475 MüKo-InsO/Nowak, § 174 Rn.26; Kübler/Prütting/Bork/Pape, § 174 Rn.33; Hess, InsO, § 174 Rn.112. 
Rücknahme der Anmeldung, im Sinne einer „anderweitigen Beendigung“ des jetzigen $\S 204$ II 1 BGB, ${ }^{476}$ lediglich die einfache Beendigung der verjährungshemmenden Wirkung ein (ex nunc). ${ }^{477}$

Palandt/Heinrichs, § 204 Rn.42; Prütting/Wegen/Weinreich/Kessler, § 204 Rn.17; MüKo-BGB/Grothe, § 204 Rn.101; Erman/J.Schmidt-Räntsch, § 204 Rn.48.

477 Wird daher eine Anmeldung nur gestellt, um in den Vorteil der durch § 204 II 1 BGB gewährten Nachfrist von sechs Monaten zu kommen, um dann zurückgenommen zu werden, weil es offensichtlich ist, dass die Forderung wegen ihrer fehlenden Qualifikation als Insolvenzforderung bestritten werden wird, liegt regelmäßig eine unzulässige Rechtsausübung i.S.d. § 242 BGB vor. Siehe etwa Palandt/Heinrichs, $\S 204$ Rn.33. 


\section{Conclusio}

Die bisherigen Erörterungen haben gezeigt, dass im Insolvenzverfahren hinsichtlich des Begriffs „Insolvenzgläubiger“ zwischen der Stellung des rein materiellrechtlichen und des formellen Insolvenzgläubigers $\mathrm{zu}$ unterscheiden ist. Jeder Gläubiger, der unter die materiellrechtliche Definition des $\S 38$ InsO fällt, wird als materiellrechtlicher Insolvenzgläubiger in das Insolvenzverfahren und dessen allgemeine Verfahrensbeschränkungen und -folgen ( $\S \S$ 89, 301 I InsO) eingebunden. Allein diese gesetzliche Einbindung begründet jedoch keine weiterführenden Verfahrensrechte des materiellrechtlichen Insolvenzgläubigers, durch die er direkten Einfluss auf das weitere Insolvenzverfahren nehmen könnte. Insofern kann man hinsichtlich seiner Verfahrensstellung von einer Verfahrensbetroffenheit sprechen, weshalb ihm hierdurch lediglich die Berechtigung zur Teilnahme an den Gläubigerversammlungen und auf rechtliches Gehör eröffnet wird. Weiterführend steht es dem materiellrechtlichen Insolvenzgläubiger aber frei, seine Insolvenzforderung gem. § 174 InsO zum Insolvenzverfahren anzumelden. Ausschließlich durch die ordnungsgemäße Insolvenzforderungsanmeldung beim zuständigen Insolvenzverwalter wird der bisher lediglich verfahrensbetroffene Insolvenzgläubiger zum formellen Verfahrensteilnehmer, wodurch ihm weitreichende Teilnahmerechte in Form des Widerspruchs- und Stimmrechts und des Anrechts auf Teilhabe an der Insolvenzmasse zugesprochen werden. Diese Differenzierung und Anknüpfung an die Anmeldung ergibt sich einerseits aus der Verfahrenssystematik der §§ 174ff. InsO und deren Bezug zur Anmeldung und andererseits aus dem insolvenzrechtlichen Verfahrensgrundsatz der Gläubigergleichbehandlung, wodurch dem einzelnen Insolvenzgläubiger nur so viel Einfluss auf das Verfahren und die Rechte der übrigen Verfahrensteilnehmer zustehen kann, wie er sich den Verfahrensrechten selbst unterwirft. Daher gilt das Anmeldeerfordernis ohne Ausnahme auch für nachrangige Insolvenzgläubiger gem. § 39 InsO.

Eine ordnungsgemäße Anmeldung hat unter Angabe der eindeutigen Bezeichnung des Verfahrens und des anmeldenden prozessfähigen Gläubigers und/oder seines ordnungsgemäßen Vertreters schriftlich und 
grundsätzlich in deutscher Sprache beim zuständigen Insolvenzverwalter zu erfolgen, wobei die sprachliche Ausnahmeregelung des Art. 42 II EuInsVO für Insolvenzgläubiger aus anderen EU-Mitgliedstaaten auch für Insolvenzgläubiger außerhalb der EU herangezogen werden sollte. Weiterhin ist der konkrete und individualisierte Lebenssachverhalt (Grund), auf dem die Insolvenzforderung beruhen soll und deren Forderungsbetrag in Euro (Betrag) anzugeben. Abschließende Voraussetzung für die ordnungsgemäße Anmeldung ist die Unterschrift des Anmeldenden (§ 130 Nr.6 ZPO) oder alternativ zur Schriftform bei - durch den Insolvenzverwalter ausdrücklich zugelassener elektronischer Übermittlung ( $§ 174$ IV InsO) - elektronischen Dokumenten in Form der qualifizierten elektronischen Signatur (§ 130a I 2 ZPO).

Die Einreichung von Dokumenten für den Bestand der angemeldeten Insolvenzforderung ist keine Voraussetzung für die Anmeldung zum Insolvenzverfahren. Zudem ist weder bei der Anmeldung noch zu einem späteren Zeitpunkt des Verfahrens zur Beweisführung hinsichtlich der materiellen Berechtigung der angemeldeten Insolvenzforderung die Einreichung von entsprechenden Urkunden im Original notwendig bzw. Voraussetzung. Alle, auch eventuell verfrüht beim vorläufigen Insolvenzverwalter eingegangene Anmeldungen sind grundsätzlich vom (vorläufigen) Insolvenzverwalter nach den voran stehenden formellen Zulassungskriterien zum Insolvenzverfahren zu prüfen. Eine materielle Prüfung der Insolvenzforderung selbst findet im Rahmen der (bloßen) Anmeldung und ihrer Zulassung zum Verfahren hingegen nicht statt, so dass jede Anmeldung, die mit der Rechtsbehauptung angemeldet wird, Insolvenzforderung zu sein, und dabei alle formellen Voraussetzungen der Anmeldung gem. § 174 InsO erfüllt, vom Insolvenzverwalter zum Verfahren zuzulassen, in die Tabelle aufzunehmen und zur Prüfung im Prüfungstermin zu stellen ist. Im weiteren Verlauf des Verfahrens ist die erstellte Tabelle ausschließlich auf der Geschäftsstelle des zuständigen Insolvenzgerichts zur Einsicht aller Verfahrensteilnehmer niederzulegen. Eine erneute bzw. zusätzliche formelle Prüfung der Anmeldung seitens des Insolvenzgerichts wird nur auf Antrag des Zurückgewiesenen rechtsbehelfsmäßig entweder im Aufsichtswege (§ 58 I, II InsO) oder unmittelbar im Prüfungstermin vorgenommen. 
Diese lediglich formelle Zulassungsprüfung des Insolvenzverwalters hat damit zweifelsohne zur Folge, dass die Hürde, Verfahrensteilnehmer zu werden, für die materiellrechtlichen Insolvenzgläubiger aber auch für eventuell unberechtigte Dritte relativ gering ist. Dies stellt für den Fortgang des Insolvenzverfahrens und der Durchsetzung der Interessen der Gläubigergemeinschaft und des Einzelnen aber keinen Nachteil dar, da allen formellen Insolvenzgläubigern die gleichen umfangreichen und teilweise fein abgestuft ( $§ 77$ InsO) geregelten Verfahrensrechte zugesprochen werden, um die berechtigte oder unberechtigte Teilnahme und Einflussnahme des Einzelnen auf das Verfahren innerhalb der Gläubigergemeinschaft umfassend klären und entsprechend feststellen zu können. Dies ist Ausdruck der Zusammenwirkung der insolvenzrechtlichen Verfahrensgrundsätze der Gläubigergleichbehandlung und der Gläubigerautonomie. Daher ist die lediglich formelle Vorprüfung der Anmeldungskriterien durch den Insolvenzverwalter im Gegenteil sachgerecht niedrig angesetzt, um den Zugang zum Verfahren für eine umfassende und letztendliche Klärung der allseitigen Verfahrensrechte der Insolvenzgläubiger zu ermöglichen, da eine anderweitige Durchsetzung eventueller Ansprüche außerhalb dieses Verfahrens gerade nicht möglich ist (§ 89 InsO).

An die Anmeldung und damit an die formelle Insolvenzgläubigerstellung knüpfen das Widerspruchsrecht gem. § 176 InsO und das ebenfalls grundsätzlich eröffnete, aber von einem eventuell erhobenen Widerspruch bzw. der Feststellung der Insolvenzforderung abhängige und daher nach

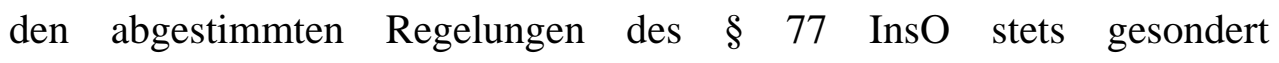
festzustellende Stimmrecht an. Des Weiteren eröffnet die Anmeldung einer Insolvenzforderung grundsätzlich das Recht zur Teilnahme an der Verteilung der Insolvenzmasse, also ein Recht auf die Quote. Inwieweit dies von dem Zeitpunkt der Anmeldung und der Erhebung eines Widerspruchs abhängig zu machen ist, wird noch im weiteren Verlauf der Arbeit zu klären sein.

Darüber hinaus kann anhand der Differenzierung zwischen angemeldeter und unangemeldeter Insolvenzforderung und der sich daraus ergebenden materiellrechtlichen oder formellen Verfahrensstellung des Insolvenzgläubigers auch hinsichtlich des Informationsbedürfnisses des 
Insolvenzgläubigers und seines Informations- bzw. Akteneinsichtsrechts während des Insolvenzverfahrens eine genaue Einordnung zu § 299 I oder II ZPO i.V.m. § 4 InsO erfolgen. Erst aufgrund seiner formellen Insolvenzgläubigerstellung wird der Insolvenzgläubiger „Partei“ des Insolvenzverfahrens i.S.d. § 299 I ZPO, so dass ihm grundsätzlich, wenn auch im Hinblick auf die informationelle Selbstbestimmung des Schuldners und des Verfahrensziels und -zwecks nicht immer uneingeschränkt, ein Recht auf Akteneinsicht und der Erteilung von entsprechenden Abschriften in Form von Kopien oder auch elektronischen Dokumenten zuzusprechen ist. Die unangemeldeten und damit lediglich materiellrechtlich verfahrensbetroffenen Insolvenzgläubiger fallen somit wie alle sonstigen Dritten unter die Regelung des § 299 II ZPO i.V.m. § 4 InsO und sind als am Verfahren unbeteiligte „Dritte“ nur insoweit berechtigt, in die Verfahrensakten Einsicht zu nehmen, wie sie ein rechtliches Interesse glaubhaft machen können. Kann ein rechtliches Interesse glaubhaft gemacht werden, so kann das Insolvenzgericht über die bloße Einsichtname hinaus aufgrund der insolvenzverfahrensrechtlichen Situation im Rahmen seiner Ermessensentscheidung im Einzelfall auch gegenüber „Dritten“ - trotz fehlender Regelung in § 299 II ZPO - die Möglichkeit der Erteilung von Abschriften in Betracht ziehen. Unabhängig von der Akteneinsicht i.S.d. § 299 ZPO steht allen Verfahrensbetroffenen die Möglichkeit offen, an den Gläubigerversammlungen teilzunehmen und sich dadurch ebenfalls, wenn auch bei weitem nicht so umfangreich wie durch eine Akteneinsicht, über den Stand des Insolvenzverfahrens zu informieren. 


\section{E. Prüfung und Feststellung der Insolvenzforderungen}

Nachdem die Grundlagen des Anmeldeverfahrens zur Teilnahme am Insolvenzverfahren und der an der Anmeldung festzumachenden Begründung von Verfahrensrechten in Teil D. herausgearbeitet worden sind, soll im folgenden Abschnitt E. das dem Anmeldeverfahren nachfolgende Prüfungs- und Feststellungsverfahren (§§ 176, 178 InsO) behandelt werden. Dabei soll zunächst auf den Prüfungstermin mit seinem grundlegenden Ablauf sowie der Möglichkeit des Widerspruchs (I.) und der nachträglichen Insolvenzforderungsanmeldung (II.) und deren Bedeutung für die Begründung von Verfahrensrechten eingegangen werden, um schließlich die Feststellung der Insolvenzforderung und ihre Wirkung (III.) zu erörtern. Innerhalb dieser Erörterungen sollen insbesondere die sich ergebenden Wechselwirkungen zwischen den einzelnen Verfahrensrechten und ihrer Ausübung sowie die Möglichkeit deren Weiterverfolgung auf prozessualem Wege gem. §§ 179ff. InsO aufgezeigt und erörtert werden, so dass am Ende dieser Arbeit ein abgestimmtes allgemeines Verfahrensmodell aufgezeigt werden kann, anhand dessen die Begründung, Ausübung und der Verlust von den sich aus dem Anmelde- und Feststellungsverfahren und dessen Ablauf ergebenden Verfahrensrechten in Einklang gebracht werden kann.

\section{Der Prüfungstermin}

Auf Grundlage der vom Insolvenzverwalter ( $\S \S 174,175$ InsO) erstellten und beim Insolvenzgericht niedergelegten (§ 175 I 2 InsO) Tabelle erfolgt im Gerichtstermin nach § 176 InsO die Prüfung der angemeldeten Insolvenzforderungen. Diese Prüfung kann dabei auch im schriftlichen Verfahren durchführt werden (§ 5 II InsO). ${ }^{478}$

Der Prüfungstermin dient dazu, die Forderungsrechte der Insolvenzgläubiger festzustellen (§ $178 \mathrm{I}$, III InsO), um eine Grundlage für die Verteilung der Insolvenzmasse nach $\S \S 187 f f$. InsO zu schaffen und den Kreis der Forderungsberechtigten für ein eventuell nachfolgendes

478 Siehe dazu unter E.II.5. (S.200ff.). 
Restschuldbefreiungsverfahren festzulegen. Dabei geht es auch um die durch (bloße) Feststellung der Insolvenzforderung erleichterte Schaffung von Vollstreckungstiteln gegen den Schuldner für die Vollstreckung nach Aufhebung des Insolvenzverfahrens, der der Schuldner nur dadurch entgegentreten kann, dass er die Forderungen erfolgreich bestreitet (§ $201 \mathrm{II}$ InsO). ${ }^{479}$ Weiter soll im Prüfungstermin geklärt werden, welche Forderungen streitig bleiben und damit nur nach Durchführung eines gerichtlichen Feststellungsverfahrens ( $\S \S$ 179ff. InsO) festgestellt und damit weiterhin im Verfahren berücksichtigt werden können. Nicht zuletzt knüpft auch das festzustellende Stimmrecht gem. $\S 77$ InsO an Widerspruch oder Insolvenzforderungsfeststellung an.

Das Prüfungs- und Feststellungsverfahren schafft demgemäß die Grundlage für die insolvenzmäßige Befriedigung sämtlicher Insolvenzgläubiger, die aufgrund ihrer besonderen Verfahrensstellung keine vollständige Befriedigung außerhalb der Zwänge des Insolvenzverfahrens, etwa als Massegläubiger oder als aus- oder absonderungsberechtigter Gläubiger, erlangen können. Insolvenzgläubiger, deren Forderungen in diesem Verfahren nicht festgestellt werden, sind bei den nachfolgenden Verteilungen nicht zu berücksichtigen (vgl. § 189 I, III InsO) und haben auch im späteren Restschuldbefreiungsverfahren keine Chance berücksichtigt zu werden (§§ 292 I 2, 294 InsO).

\section{Prüfungstermin - Berichtstermin}

Der allgemeine ${ }^{480}$ Prüfungstermin wird bereits im Eröffnungstermin bestimmt (§ 29 I Nr.2 InsO) und hat spätestens fünf Monate nach Eröffnung des Insolvenzverfahrens stattzufinden. ${ }^{481}$ Eine Ladung der Beteiligten ist wegen der erfolgten Zustellung des Eröffnungsbeschlusses

Uhlenbruck/Uhlenbruck, § 176 Rn.1; Kübler/Prütting/Bork/Pape, § 176 Rn.4; MüKoInsO/Nowak, § 176 Rn.1 m.w.N.

$480 \mathrm{Zu}$ eventuell nachfolgenden Prüfungsterminen siehe unter E.II.4. (S.198ff.).

481 Diese Frist ergibt sich aus der Höchstfrist für die Anmeldungen der Insolvenzforderungen (3 Monate, § 28 I 2 InsO) und dem Höchstabstand zwischen dem Ablauf der Anmeldefrist und dem Prüfungstermin (2 Monate, § 29 I Nr.2 InsO). Siehe bereits unter D.IV.7. (S.72ff.). 
(§ 30 II InsO) und wegen der öffentlichen Bekanntmachung (§ 9 III InsO) nicht erforderlich. ${ }^{482}$

Aus der gesetzlichen Konzeption der vorgegebenen Terminierungen ergibt sich, das der Prüfungstermin (§ 29 I Nr.2 InsO) generell nicht vor dem Berichtstermin stattfindet ( $§ 29$ I Nr.1 InsO), aber mit diesem verbunden werden kann (§ 29 II InsO). Da beide Termine jedoch Gläubigerversammlungen sind und im Berichtstermin auf der Grundlage eines Berichts des Insolvenzverwalters über den Fortgang des Insolvenzverfahrens bereits Beschlüsse gefasst werden können/sollen, stellt sich die Frage, welche Anforderungen an die Anmeldung und damit an eine Stimmberechtigung zu stellen sind, wenn der Berichtstermin (a) vor dem Prüfungstermin und (b) zudem noch vor Ablauf der Anmeldefrist liegt.

\section{a) Stimmrecht angemeldeter ungeprüfter Insolvenzforderungen}

§ 77 I 1 InsO setzt im Gegensatz zu § 95 I 1 KO, der auch die Feststellung der Forderung verlangt, für eine Stimmberechtigung nur die Anmeldung und das fehlende Bestreiten voraus, wobei die Anmeldung und ihre Voraussetzungen ausdrücklich in $\S 174$ InsO geregelt sind. Daraus folgt, dass angemeldete, aber noch nicht geprüfte Insolvenzforderungen mit dem vollen Betrag (vorläufig) stimmberechtigt sein müssen, soweit sie bzw. das Stimmrecht nicht bestritten wurde. ${ }^{483}$ Diese Auslegung entspricht sowohl der Systematik des Gesetzes als auch dem Sinn und Zweck des § 77 InsO. Die Regelung des $\S 77$ InsO gilt hinsichtlich der Festlegung des Stimmrechts für alle Gläubigerversammlungen, also nicht nur für solche ab dem Prüfungstermin. Es muss aufgrund dieser Norm also möglich sein, auch Insolvenzforderungen, die noch nicht geprüft sein können, in eine

Siehe bereits unter D.III. (S.33ff.).

83 BGH ZIP 2004, 2339 (2339); LG Göttingen ZIP 1989, 1481 (1481); Uhlenbruck/Uhlenbruck, § 77 Rn.10; BK/Blersch, § 77 Rn.3; HK/Eickmann, § 77 Rn.3; Kübler/Prütting/Bork/Kübler, § 77 Rn.4; Haarmeyer/Wutzke/Förster, InsO, Kap.6 Rn.61; Huntemann/Brockdorff/Buck, Kap.10 Rn.91; Müko-InsO/Ehricke, § 77 Rn.4 m.w.N. 
Stimmrechtsentscheidung einzubeziehen, ${ }^{484}$ um den Insolvenzgläubigern dieser Forderungen die Ausübung des unmittelbar an die formelle Insolvenzgläubigerstellung anknüpfenden Stimmrechts zu ermöglichen. Die Unsicherheit, dass die noch ungeprüfte Insolvenzforderung nach angegebenem Grund und angegebener Höhe nicht bestehen könnte, wird im Bestreitensfall über das Verfahren nach § 77 II InsO beseitigt, ${ }^{485}$ der in Anknüpfung an Absatz I eben nicht nur festgestellte, sondern generell „angemeldete Forderungen“ und damit eben auch die ungeprüften Forderungen erfasst. Das Nicht-/Bestreiten der Insolvenzforderung bezieht sich im Fall der ungeprüften Forderungen jedoch nicht auf den Prüfungstermin oder das schriftliche Prüfungsverfahren, sondern nur auf die Gläubigerversammlung, in der das Stimmrecht ausgeübt werden soll, da insoweit die Prüfung der eigentlichen Insolvenzforderungen und ihrer materiellen Berechtigung noch nicht stattgefunden haben kann. ${ }^{486}$ Dies zeigt, dass das Bestreiten im Sinne des § 77 I InsO nicht gleichbedeutend mit dem Bestreiten zur Insolvenztabelle nach § 176 Satz 2 InsO ist, sondern dass $\S 77$ InsO generell das Stimmrecht bestrittener Forderungen $^{487}$ und damit auch das Stimmrecht bisher ungeprüfter Insolvenzforderungen regelt. Im Umkehrschluss kann bzw. wird bei einem Widerspruch gegen eine ungeprüfte Forderung hinsichtlich des Stimmrechts nicht etwa zugleich der feststellungshindernde Widerspruch im Sinne des $\S 176$ InsO gegen die Forderung erhoben bzw. für den späteren Prüfungstermin vorweggenommen, da es im Fall der ungeprüften Insolvenzforderung nur um die Feststellung des Stimmrechts und eben nicht (auch) um die Feststellung der Insolvenzforderung zur Tabelle geht. Daraus ergibt sich, dass das so festgestellte Stimmrecht einer ungeprüften, aber auch einer bisher allgemein nicht gem. § 178 InsO festgestellten Insolvenzforderung regelmäßig nur vorläufiger Natur ist und nur so lange Bestand hat, bis es durch einen (erneuten) Widerspruch des

\footnotetext{
484 Dies folgt zudem aus der ausdrücklichen Einbeziehung des § 95 I und II KO in § 77 InsO, wobei nach $\S 95$ II $\mathrm{KO}$ auch die ungeprüften Forderungen grundsätzlich stimmberechtigt waren. - Siehe Begr. zu $\S 88$ (§ 77 InsO) RegE, BT-Drucks. 12/2443, S.133.

485 Siehe schon D.VI.2.a) (S.99ff.).

486 LG Göttingen NZI 2000, 490 (490); AG Hamburg NZI 2000, 138 (138); Uhlenbruck/Uhlenbruck, § 77 Rn.10 m.w.N.

487 So auch Uhlenbruck/Uhlenbruck, § $77 \mathrm{Rn} .10$.
} 
Insolvenzverwalters ${ }^{488}$ oder stimmberechtigten Insolvenzgläubigers in Frage gestellt wird und nach

$\S 77$ II InsO eine Einigung oder mangels Einigung eine Entscheidung des Insolvenzgerichts herbeizuführen ist. ${ }^{489}$

In späteren Gläubigerversammlungen sind Insolvenzforderungen dann als nicht geprüft anzusehen, wenn die Prüfung bisher nicht erfolgen konnte, also etwa dann, wenn sie nach Ablauf der Anmeldefrist angemeldet wurden und ihre Mitprüfung wegen eines Widerspruchs des Insolvenzverwalters oder eines Insolvenzgläubigers gegen die Prüfung im ordentlichen Prüfungstermin nicht erfolgen konnte, ${ }^{490}$ oder wenn wegen einer erst nach dem Prüfungstermin erfolgten Anmeldung ein besonderer Prüfungstermin bestimmt oder die Prüfung im schriftlichen Verfahren angeordnet worden ist und noch nicht stattgefunden hat (vgl. § 177 I InsO). ${ }^{491}$

\section{b) Stimmrecht bisher unangemeldeter Insolvenzforderungen}

Fraglich ist jedoch, was hinsichtlich einer Stimmberechtigung gelten muss, wenn bisher keine formelle Anmeldung der Insolvenzforderung gem. § 174 InsO stattgefunden hat und auch nicht stattfinden musste, weil die erste Gläubigerversammlung vor Ablauf der Anmeldefrist liegt.

Dem Wortlaut des $\S 77$ I 1 InsO nach (,angemeldet...worden sind“) ist grundsätzlich davon auszugehen, dass diejenigen Forderungen kein Stimmrecht haben, die erst in der Gläubigerversammlung benannt werden.

488 Dies gilt auch für einen Widerspruch des Schuldners in Eigenverwaltung. Siehe dazu bereits unter D.VI.2.b) (S.102ff.).

489 Hiervon zu differenzieren ist ein Stimmrechtsverbot. Einer zu befürchteten Ausübung des Stimmrechts durch sachfremde Beweggründe ist nicht durch eine generelle Stimmrechtsversagung, sondern dadurch zu begegnen, dass das Insolvenzgericht den Gläubiger von der Teilnahme an der betreffenden Abstimmung wegen Interessenskollision ausschließt. Auch wenn ein solches Stimmrechtsverbot in der Insolvenzordnung selbst nicht geregelt ist, folgt aus dem in § 34 BGB, § 136 I AktG, $\S 47$ IV GmbHG, § 43 VI GenG zum Ausdruck kommenden allgemeinen Rechtsgedanken, dass man in Einzelfällen von einer Abstimmung in eigener Sache ausgeschlossen werden kann. Auf Einzelheiten soll im Rahmen dieser Arbeit jedoch nicht weiter eingegangen werden. - H.M. siehe etwa AG Kaiserslautern ZIP 2006, 531 (532); Kübler/Prütting/Bork/Kübler, § 77 Rn.20f.; MüKo-InsO/Ehricke, § 77 Rn.35ff. m.w.N.

490 Siehe dazu unter E.II.2. (S.189ff.).

491 Siehe auch Kübler/Prütting/Bork/Kübler, § 77 Rn.11; MüKo-InsO/Ehricke, § 77 Rn.5. 
Dieses Verständnis wird gestützt durch Sinn und Zweck der Regelung: Zum einen wird durch vorherige Anmeldung gem. § 174 InsO die Absicht der Teilnahme am Verfahren wirksam bekundet, und zum anderen soll damit eine zügige Durchführung der Versammlung gewährleistet werden, weil dann bereits bei Beginn der Versammlung der Kreis der gegebenenfalls stimmberechtigten Insolvenzgläubiger feststeht und insoweit möglicherweise umfassende und langwierige formale Prüfungen im Termin vermieden werden. ${ }^{492}$ Betrachtet man andererseits das Anmeldeerfordernis des $\S 77$ InsO in seinem engen Zusammenhang mit $\S 28$ InsO und § 174 InsO, so bedarf es hinsichtlich des besonderen Falls, dass die erste Gläubigerversammlung vor dem Ende der benannten Anmeldefrist ( $§ \quad 28$ I InsO) stattfindet, einer Ausnahme von der grundsätzlich im Vorhinein zu erfolgenden Anmeldung für eine mögliche Stimmberechtigung. Hier darf es dem Insolvenzgläubiger nicht zum Nachteil gereichen, dass er die Frist für seine Anmeldung ausschöpfen wollte und er daher zum Zeitpunkt der Gläubigerversammlung seine Insolvenzforderung noch nicht gem. § 174 InsO angemeldet hat, da er in seinem Vertrauen zu schützen ist, keine Rechte zu verlieren, wenn und solange er die vom Insolvenzgericht gesetzte Frist ausschöpft. Zudem wäre es nicht mit dem Grundsatz der Gläubigerautonomie zu vereinbaren, wenn einige Insolvenzgläubiger von der gegebenenfalls bestehenden Notwendigkeit ausgeschlossen würden, schnell und frühzeitig - also noch vor Ende der Anmeldefrist - in der Lage zu sein, wichtige Entscheidungen in der Gläubigerversammlung zu treffen (z.B. die Wahl eines anderen Insolvenzverwalters gem. § 57 InsO), weil sie ihre Insolvenzforderungen gem. $\S 174$ InsO bis dato nicht angemeldet haben. ${ }^{493}$ Liegt daher der Termin der Gläubigerversammlung vor dem Ende der Anmeldefrist, so ist es für das Anmeldeerfordernis des $\S 77$ I InsO ausreichend, wenn der Insolvenzgläubiger erst zu Beginn der betreffenden Verhandlung seine Forderung beim grundsätzlich anwesenden ${ }^{494}$ Insolvenzverwalter anmeldet. Eine bloße mündliche Geltendmachung der Forderung seitens des

92 MüKo-InsO/Ehricke, § 77 Rn.6.

493 AG Hamburg NZI 2000, 138 (139); Uhlenbruck/Uhlenbruck, § 77 Rn.3; Kübler/Prütting/Bork/Kübler, § 77 Rn.29; FK/Kind, § 77 Rn.4; MüKo-InsO/Ehricke, $\S 77$ Rn.6; Nerlich/Römermann/Delhaes, § 77 Rn.3; Huntemann/Brockdorff/Buck, Kap.10 Rn.92;

494 Dazu unter E.I.2.c) (S.136f.). 
Insolvenzgläubigers ist dabei durch den eindeutigen Wortlaut des § 174 I 1 InsO, der eine schriftliche Anmeldung beim Insolvenzverwalter fordert, jedoch nicht ausreichend. ${ }^{495}$ Denn $\S 77$ InsO knüpft nicht an eine Geltendmachung, sondern an die Anmeldung i.S.d. § 174 InsO und der sich dadurch ergebenden formellen Insolvenzgläubigerstellung des Anmeldenden an. Zudem würde verkannt werden, dass es nicht nur um die Stimmberechtigung in dieser einen Gläubigerversammlung geht, sondern letztendlich um den Willen, am gesamten Insolvenzverfahren teilzunehmen. Die Ausnahme zur Regel liegt in dem hier vorliegenden besonderen Fall nur in einer zuzulassenden „Ad-hoc-Anmeldung“ und deren unmittelbaren Berücksichtigung bezüglich eines Stimmrechts in der Gläubigerversammlung und nicht auch hinsichtlich der sonstigen unproblematisch vor Ort erfüllbaren Voraussetzungen der Anmeldung des $\S 174$ InsO. Sollte der Anmeldende dabei nicht in der Lage sein, ein einfaches Anmeldeformular auszufüllen, wird er auch keine Insolvenzforderung behaupten können. Die Insolvenzforderung muss schließlich nachvollziehbar nach Grund und Betrag beziffert sein, da ansonsten ein Stimmrecht nicht feststellbar ist bzw. die übrigen stimmberechtigten Insolvenzgläubiger und der Insolvenzverwalter keine Möglichkeit haben, sich auf ein Stimmrecht in Form eines bestimmten Betrages zu einigen. Zudem muss die (ordnungsgemäße) Forderungsanmeldung in die Tabelle und zu den Unterlagen aufgenommen werden können; dies wäre ohne eine schriftliche Anmeldung gem. § 174 InsO jedoch nicht möglich (§ 175 I 1 InsO). Sollte die Forderung von den anwesenden Beteiligten gleichwohl bestritten werden, findet für die Festlegung einer Stimmberechtigung wiederum § 77 II InsO Anwendung. Somit bleibt festzuhalten, dass einem Insolvenzgläubiger grundsätzlich nur dann im dem Prüfungstermin vorgezogenen Berichtstermin ein (vorläufiges) Stimmrecht zusteht, wenn er seine Insolvenzforderung, unabhängig vom Ablauf der Anmeldefrist, spätestens im Termin gem. $\S 174$ I InsO beim anwesenden Insolvenzverwalter anmeldet.

495 So aber Uhlenbruck/Uhlenbruck, § 77 Rn.3; Kübler/Prütting/Bork/Kübler, § 77 Rn.29; MüKo-InsO/Ehricke, § 77 Rn.6; wie hier auch HK/Mäusezahl, § 77 Rn.2; Jaeger/Weber, KO, § 95 Rn.5. 


\section{Teilnahme am Prüfungstermin}

Der Prüfungstermin ist eine Gläubigerversammlung (§ 29 I Nr.2 InsO). Zur Teilnahme an Gläubigerversammlungen sind - auch ohne Forderungsanmeldung $^{496}$ - gem. § 74 I 2 InsO grundsätzlich alle absonderungsberechtigten Gläubiger (vgl. § 76 II 2.Halbsatz InsO), alle (auch nachrangige) Insolvenzgläubiger, der Insolvenzverwalter, die Mitglieder des Gläubigerausschusses und der Schuldner berechtigt. Aus den beiden Regelungen ( $\S \S 74,76$ InsO) zu entnehmenden abschließenden Aufzählungen der Teilnahmeberechtigten und aus der Tatsache, dass der Insolvenzrichter bzw. Rechtspfleger gerade nicht als erkennender Richter im Sinne von. § 169 GVG tätig wird, ist zu schließen, dass der Termin als Gläubigerversammlung lediglich als parteiöffentlich einzustufen ist. ${ }^{497}$ Gegen eine grundsätzlich öffentliche Gläubigerversammlung ${ }^{498}$ sprechen zudem nicht nur die besondere insolvenzverfahrensrechtliche Situation des Schuldners hinsichtlich dessen Recht auf informationelle Selbstbestimmung, sondern auch die insolvenzrechtlichen Interessen der Insolvenzgläubiger, wichtige Informationen, insbesondere im Fall der Aufstellung eines Insolvenzplans und/oder der Fortführung eines Unternehmens, zur Erreichung des jeweiligen Verfahrenszwecks verfahrensintern zu halten. ${ }^{499}$ Darüber hinaus erscheint es aufgrund des bloß parteiöffentlichen Termins auch wesentlich einfacher, ausnahmsweise einzelnen Personen die Anwesenheit in der Gläubigerversammlung durch das Insolvenzgericht zu gestatten ( $\$ 175$ II 1 GVG), als umgekehrt - bei der Annahme eines öffentlichen Termins - zu Beginn einer jeden Gläubigerversammlung aufgrund der besonderen insolvenzrechtlichen Verfahrensinteressen zunächst Ausschlüsse von der Teilnahme auszusprechen. Wer zur Teilnahme berechtigt ist und wer nicht, ergibt sich aus den Gläubigerverzeichnissen, der Tabelle bzw. den Anmeldungen und

Siehe bereits unter D.VI.1. (S.94ff).

497 Das Insolvenzverfahren ist kein Erkenntnisverfahren. Siehe bereits D.I. (S.23ff.). - Uhlenbruck/Uhlenbruck, § 176 Rn.3; Braun/Specovius, § 176 Rn.4; HK/Irschlinger, § 176 Rn.3; MüKo-InsO/Ehricke, § 74 Rn.30 m.w.N.

498 So aber MüKo-InsO/Nowak, § 176 Rn.3, der bei einem Ausschluss der Öffentlichkeit - ohne nähere Begründung - lediglich auf ein Zuwiderlaufen des Sinns und Zwecks des Prüfungstermins und auf sich eventuell stellende Probleme bei der Feststellung der Präsenz abstellt. Letzteres macht jedoch keinen Unterschied hinsichtlich der dann umgekehrt auszusprechenden Ausschlüsse vom Termin und greift daher nicht. 
kann bei Unstimmigkeiten darüber hinaus durch Rückfragen beim im Prüfungstermin anwesenden Schuldner ${ }^{500}$ und Insolvenzverwalter geklärt werden.

\section{a) Insolvenzgläubiger}

Die Teilnahme der Insolvenzgläubiger am Prüfungstermin ist freiwillig. ${ }^{501}$ Die Insolvenzforderungen werden auch geprüft, wenn die Gläubiger der zu prüfenden Insolvenzforderungen nicht anwesend sind. ${ }^{502}$ Nicht anwesende Insolvenzgläubiger, deren angemeldeten Forderungen im Prüfungstermin ordnungsgemäß widersprochen wurde, sind vom Insolvenzgericht zu benachrichtigen (vgl. § 179 III 3 InsO).

\section{b) Schuldner}

Der Schuldner ist grundsätzlich nicht verpflichtet, am Prüfungstermin teilzunehmen (vgl. § 186 I InsO). Nach § 97 I InsO ist der Schuldner ${ }^{503}$ jedoch verpflichtet, dem Insolvenzgericht, dem Insolvenzverwalter, dem Gläubigerausschuss und auf Anordnung des Insolvenzgerichts der Gläubigerversammlung über alle das Verfahren betreffenden Verhältnisse Auskunft zu geben. Hierzu gehören auch die Auskunft über Grund und Höhe der angemeldeten Insolvenzforderungen und, da auch der Prüfungstermin eine Gläubigerversammlung ist und die Insolvenzforderungen „mündlich“ geprüft ${ }^{504}$ werden, ist die Anwesenheit des Schuldners in der Regel unverzichtbar. Daher sollte das Insolvenzgericht regelmäßig gehalten sein, von der gesetzlich ausdrücklich vorgesehenen Möglichkeit Gebrauch zu machen, das persönliche Erscheinen des Schuldners im Prüfungstermin gem. § 97 III 1 InsO

\footnotetext{
Siehe dazu nachfolgend unter E.I.2.b) (S.135f.).

KO-Motive, S.362 = Hahn, KO, S.325.

2 Diese Regelung ergäbe sich schon aus § $176 \mathrm{~S} .1$ InsO und entspreche ohnehin der bisherigen Rechtslage (§ $143 \mathrm{KO}$ ). So Begr. des Rechtsausschusses zu § 203 (§ 176 InsO) RegE, BT-Drucks. 12/7302, S.178.

503 Für die organschaftlichen Vertreter eines Schuldnerunternehmens oder die vertretungsberechtigten persönlich haftenden Gesellschafter des Schuldners gilt § 101 I 1 InsO.

504 Siehe dazu unter E.I.3. (S.137ff.) und E.I.4.c)aa) (S.144ff.).
} 
anzuordnen, um dringende benötigte bzw. fehlende Auskünfte des Schuldners einzuholen und Verzögerungen vorzubeugen. ${ }^{505}$ Der Schuldner kann gem. § 98 II InsO auch zwangsweise vorgeführt werden.

\section{c) Insolvenzverwalter}

Fraglich ist, ob für den Insolvenzverwalter eine Anwesenheitspflicht im Prüfungstermin besteht.

Eine ausdrückliche gesetzliche Regelung hinsichtlich einer Anwesenheitspflicht fehlt. Hieraus wird teilweise geschlossen, ${ }^{506}$ dass der Insolvenzverwalter auch einen Dritten bevollmächtigen könne, der ihn vertritt bzw. sich seinen Anweisungen entsprechend zu den angemeldeten Insolvenzforderungen erklärt. Diese Ansicht kann aber schon dann nicht mehr überzeugen, wenn man bedenkt, dass der Insolvenzverwalter oftmals erstmalig im Prüfungstermin Erklärungen abzugeben und Entscheidungen zu treffen hat, die sich erst aus dem Verlauf des Termins ergeben, etwa wenn es um die Festsetzung von Stimmrechten geht. Dass der Bevollmächtigte nicht befugt ist, über die Vorgaben des Insolvenzverwalters hinaus Erklärungen $\mathrm{zu}$ den angemeldeten Insolvenzforderungen abzugeben, ist dabei unbestritten. ${ }^{507}$ Die Befugnis zur Einschaltung eines Bevollmächtigten muss nämlich dort eingeschränkt werden, wo es nicht um die Regelung eigener Angelegenheiten geht oder solcher Angelegenheiten, bei denen man selbst nur für einen anderen handelt, sondern um die Ausübung einer im öffentlichen oder im Drittinteresse verliehenen, an die Person geknüpften höchstpersönlichen Rechtsmacht. ${ }^{508}$ Mit dieser Rechtsmacht, die eben insoweit nicht autonom ist, korrespondiert die Rechtspflicht zu ihrer sachgerechten Ausübung. Was nur ein Insolvenzverwalter tun kann, muss nicht nur letztlich ihm zurechenbar sein, sondern muss auch auf seine persönliche Willensbildung

505 Uhlenbruck/Uhlenbruck, $\S \quad 176 \quad$ Rn.5; HK/Irschlinger, $\quad \S \quad 176 \quad$ Rn.2; Gottwald/Eickmann, § 63 Rn.47; KS/Eckardt, S.756 Rn.24; FK/Kießner, § 186 Rn.1.

506 Hess, InsO, § 176 Rn.12f.; BK/Breutigam, § 176 Rn.7; Gottwald/Eickmann, § 63 Rn.46; FK/Kießner, § 176 Rn.5; Bratvogel, KTS 1977, 229 (231); Eickmann, KTS 1986, 197 (204).

507 Siehe etwa Eickmann, KTS 1986, 197 (203f).

508 Zur Insolvenzverwalterstellung als höchstpersönliches Amt siehe nur Uhlenbruck/Uhlenbruck, § 56 Rn.24 m.w.N. 
zurückzuführen sein. Wäre dies anders, so handelte es sich letztlich um eine unzulässige partielle Übertragung des Amtes auf einen anderen. ${ }^{509}$ Die Prüfung und Erörterung der Insolvenzforderungen sind dabei insolvenztypische und originäre Aufgaben des Insolvenzverwalters, dem die ordnungsgemäße Verwaltung der Insolvenzmasse im Rahmen seiner Amtsstellung gem. § 80 I InsO übertragen wurde; sie können daher nicht delegiert werden. ${ }^{510}$ Dies wird durch die nunmehrige Verlagerung der ausschließlichen Anmeldung und Tabellenerstellung vom Insolvenzgericht hin zum Insolvenzverwalter umso deutlicher. Der Insolvenzverwalter ist der Einzige, dem sämtliche Umstände des Verfahrens sowohl von Seiten des Schuldners als auch hinsichtlich der Belange der Insolvenzgläubiger bekannt sind. Dem Insolvenzverwalter kommt daher auch praktisch eine überragende Bedeutung $\mathrm{zu}$, die seine persönliche Anwesenheit im Prüfungstermin unerlässlich macht. Sollte der Insolvenzverwalter daher zum Prüfungstermin unabkömmlich sein, ist der Prüfungstermin vom Insolvenzgericht zu verlegen (§§ 136 III, 227 ZPO i.V.m. § 4 InsO). Dies gilt ebenso für den Sachwalter ( $§ 270$ I, III InsO), den Treuhänder (§§ 313 I, 292 InsO) und Eigenverwalter ${ }^{511}$ (§ 283 InsO), denen die Aufgaben des Insolvenzverwalters entsprechend übertragen sind.

\section{Leitung und Durchführung des Prüfungstermins}

Der Prüfungstermin wird vom Insolvenzgericht geleitet (§ 76 I InsO) und protokolliert (§ 178 II 1 InsO). In aller Regel wird dies durch den Rechtspfleger geschehen (§ 18 I RPflG), wenn nicht der zuständige Richter die Leitung des Termins an sich gezogen hat (§ 18 II RPflG).

Im Gegensatz zur vorangegangen (lediglich) formellen Prüfung der Anmeldung durch den Insolvenzverwalter sollen im Prüfungstermin gem.

509 Ausführlich dazu Eickmann, KTS 1986, 197 (202ff.).

510 So schon KO-Motive, S.362 = Hahn, KO, S.325: „...der Verwalter [als] ...gemeinschaftlicher Kontradiktor [ist] in Wahrnehmung der Interessen aller Gläubiger [dazu] berufen..., jede einzelne Forderung...zu prüfen. ... Ohne den Verwalter kann [im Prüfungstermin] nicht verhandelt werden.“; LG Trier NZI 2006, 243 (244); AG Hohenschönhausen ZInsO 2000, 168 (168); Braun/Specovius, § 176 Rn.4; Kübler/Prütting/Bork/Pape, § 176 Rn.8; KS/Eckardt, S.754f. Rn22; MüKoInsO/Nowak, $\quad \S \quad 176 \quad$ Rn.7; $\quad$ Uhlenbruck/Uhlenbruck, $\$ 176 \quad$ Rn.8; Haarmeyer/Wutzke/Förster, Kap.7 Rn.44; HK/Irschlinger, § 176 Rn.2; FK/Kießner, § 176 Rn.5; Graf-Schlicker/Graf-Schlicker, § 176 Rn.3.

511 Siehe auch unter E.I.4.d)aa) (S.157ff.). 
$\S 176 \mathrm{~S} .1$ InsO alle angemeldeten Insolvenzforderungen ihrem Betrag und ihrem Rang nach geprüft werden. Der Wortlaut des § 176 InsO ist insofern leicht missverständlich, als von einer scheinbar erst im Termin selbst vorzunehmenden Prüfung der Insolvenzforderung die Rede ist. Denn die eigentliche materiellrechtliche Prüfung der angemeldeten Insolvenzforderungen im Sinne des $\S 176$ S.1 InsO findet durch den Insolvenzverwalter und alle Widerspruchsberechtigten grundsätzlich bereits im Vorfeld durch Einsichtnahme in die ausgelegte Tabelle und die übrigen eingereichten Urkunden und Beweisstücke statt (§§ 174 I 2, 175 I 2 InsO). ${ }^{512}$ Im Prüfungstermin selbst wird vielmehr „nur noch“ das Ergebnis dieser bereits erfolgten Prüfung der Insolvenzforderungen durch die widerspruchsberechtigten Verfahrensbeteiligten verlautbart und durch das Insolvenzgericht entsprechend protokolliert bzw. festgestellt (§ 178 II 2 InsO). ${ }^{513}$ Die ausschließliche Prüfung durch die Widerspruchsberechtigten ergibt sich dabei aus $\S 176$ S.2 InsO. Eine Prüfung der Insolvenzforderungen durch das Insolvenzgericht findet wie bereits erörtert ${ }^{514}$ nicht statt. Rangfragen spielen nur bei der zugelassenen Anmeldung von nachrangigen Insolvenzforderungen ( $\S \S 39,327$ InsO) eine Rolle.

Auch wenn $\S 176$ InsO dies nicht ausdrücklich vorsieht, wird hinsichtlich des Umfangs der materiellrechtlichen Prüfung neben Betrag und Rang gleichwohl auch der Grund der Insolvenzforderung (summarisch) Gegenstand der Prüfung sein, da dieser überhaupt die Grundlage des („nur“) zu prüfenden Betrages und des Rangs der angemeldeten Insolvenzforderung ist. ${ }^{515}$ Ohne Bezeichnung des Grundes wäre die Insolvenzforderung gar nicht identifizierbar. Die Prüfung des Einen geht mit der des Anderen unvermeidbar einher und umfasst daher qualitativ „den vollen Umfang der Forderung “516. Dies wird zudem mittelbar durch $\S 181$ InsO deutlich, wo die drei Kategorien für die Feststellung zur

Siehe auch unter E.I.4.c)aa) (S.144ff.).

Vgl. sinngemäß auch KO-Motive, S.362 = Hahn, KO, S.325: „[Die Prüfung und Erörterung] geschieht aber nur, um festzustellen, was für Konkursforderungen erhoben, welche Forderungen von maßgeblicher Seite anerkannt und welche bestritten werden."

514 Siehe ausführlich schon unter D.IV.5.a) (S.53ff.).

515 Braun/Specovius, § 176, Rn.7; Nerlich/Römermann/Becker, $\S 176$ Rn.24; Kuhn/Uhlenbruck, KO, § 141 Rn.1.

516 KO-Motive, S.384 = Hahn, KO, S.343. 
Tabelle außerhalb des Insolvenzverfahrens nach Bestreiten durch einen Widerspruchsberechtigten aufgeführt sind (,...Grund, Betrag und Rang...wie die Forderung in der Anmeldung oder im Prüfungstermin bezeichnet worden ist“). Insofern ist also unter Prüfung im Sinne des $§ 176$ S.1 InsO eine umfassende materiellrechtliche Prüfung der angemeldeten Insolvenzforderungen hinsichtlich ihrer Berechtigung zur Teilnahme am Insolvenzverfahren zu verstehen.

Um den Ablauf des Termins zu straffen und insbesondere in Großverfahren eine Beschleunigung des Verfahrens zu erreichen, sind - im Gegensatz zur Konkursordnung (vgl. § 141 I KO) - nicht mehr alle angemeldeten Insolvenzforderungen einzeln zu erörtern, sondern gem. $\S 176$ S.2 InsO nur noch die jeweils bestrittenen. Die übrigen Insolvenzforderungen werden daher nur pauschal aufgerufen, ${ }^{517}$ um die Erhebung von (weiteren) Widersprüchen zu ermöglichen und in der Tabelle vermerken zu können. ${ }^{518}$

Aus § 176 S.2 InsO ergibt sich zudem die grundsätzliche Mündlichkeit des Prüfungstermins. Das Prinzip der Mündlichkeit kann jedoch durch $\S 5$ III 1 InsO eingeschränkt bzw. ganz durch ein schriftliches Verfahren (§ 5 II 1 InsO) ersetzt werden. ${ }^{519}$ Unter der Erörterung im Sinne des $\S 176$ S.2 InsO ist jedoch nicht eine umfassende Diskussion oder mündliche Verhandlung im Sinne des $\S 128$ ZPO zu verstehen, wie es das Erkenntnisverfahren vorsieht (§§ 279ff. ZPO). ${ }^{520}$ Die mündliche Erörterung der bestrittenen Forderung dient lediglich der Klärung der Streitpunkte unter den Beteiligten und somit der Vermeidung unsinniger Feststellungsklagen zur Tabelle. ${ }^{521}$ Diese, zunächst den Widerspruchsberechtigten untereinander eingeräumte (gütliche) Erörterungsmöglichkeit, folgt aus der gegenseitigen Prüfungs- und An- bzw. Aberkennungs-

517 Graf-Schlicker/Graf-Schlicker, § 176 Rn.8; Kübler/Prütting/Bork/Pape, § 176 Rn.1.

518 Vgl. auch Begr. zu $\S 203$ (§ 176 InsO) RegE, BT-Drucks. 12/2443, S.184: „Die Vorschrift schließt aber nicht aus, daß der Insolvenzverwalter oder ein Gläubiger erst im Verlauf des Prüfungstermins eine Forderung bestreitet; hierzu muß das Insolvenzgericht auch Gelegenheit geben.“ Die ausdifferenzierte Formulierung des $\S 176$ InsO lässt daher die Ansicht, dass die bisher unbestrittenen Insolvenzforderungen überhaupt nicht aufgerufen werden, nicht zu. So aber Uhlenbruck/Uhlenbruck, $\S 176$ Rn.14.

519 Dazu unter E.II.5. (S.200ff.).

520 KO-Motive, S.361 = Hahn, KO, S.324; siehe bereits unter D.I. (S.23ff.) und D.IV.5.a) (S.53ff.).

521 MüKo-InsO/Nowak, § 176 Rn.1. 
kompetenz über die weitere Teilnahme des jeweils anderen Insolvenzgläubigers mit seiner Forderung am Verfahren und ist wiederum Ausdruck der Gläubigerautonomie. Lässt sich der Grund für einen Widerspruch nicht ausräumen, hat eine Feststellung im dafür vorgesehenen ordentlichen Streitverfahren nach den $\S \S$ 179, 184 InsO zu erfolgen. Insofern kann auch das Aufklärungsgebot (§ 139 ZPO i.V.m. § 4 InsO) des Insolvenzgerichts innerhalb des Prüfungstermin nur so weit reichen, wie die angebrachten Streitpunkte einer sofortigen Klärung im Prüfungstermin zugänglich sind. ${ }^{522}$ Die Erörterung kann nur bis zu dem Punkt gehen, an dem der Widersprechende einlenkt oder bekundet, endgültig bei seinem Widerspruch zu bleiben.

Sollte gerade in Großverfahren die Prüfung und Erörterung aller angemeldeten Insolvenzforderungen in einem Prüfungstermin nicht möglich sein, kann dieser von Amts wegen oder auf Antrag gem. § 74 II 2 InsO vertagt werden. ${ }^{53}$ Das Insolvenzgericht bestimmt dann einen weiteren Termin zur Fortsetzung der Prüfung. Eine öffentliche Bekanntmachung des weiteren Prüfungstermins kann dabei aufgrund der unmittelbaren Bekanntgabe an die anwesenden Beteiligten im Prüfungstermin unterbleiben (§ 74 II 2 InsO). Hiervon zu unterscheiden ist der besondere Prüfungstermin im Sinne von $\S 177$ I 2 InsO, der noch gesondert zu untersuchen sein wird. ${ }^{524}$

\section{Der Widerspruch}

Nach § 178 I InsO gilt eine zur Prüfung gelangte Insolvenzforderung insoweit als festgestellt und dessen Inhaber damit zur Teilnahme am Verfahren und Teilhabe an der Insolvenzmasse berechtigt (vgl. §§ 187ff., 292 I 2 InsO), wie sie im Prüfungstermin vom Insolvenzverwalter oder einem Insolvenzgläubiger unwidersprochen bleibt. ${ }^{525}$ Der Widerspruch des

\footnotetext{
522 So schon KO-Motive, S.361 = Hahn, KO, S.325; Nerlich/Römermann/Becker, § 176 Rn.26; MüKo-InsO/Nowak, § 176 Rn.2.

523 LG Göttingen ZIP 1989, 1471 (1471); MüKo-InsO/Nowak, § 176 Rn.13ff.; KS/Eckardt, S. 759 Rn.32; Uhlenbruck/Uhlenbruck, § 176 Rn.17 m.w.N.

524 Dazu ab E.II. (S.187ff.).

525 Dazu auch ab E.III. (S.206ff.).
} 
Schuldners - außer in der Eigenverwaltung ${ }^{526}$ - hat gem. § 201 II InsO hingegen nur eine vollstreckungshindernde Wirkung nach beendetem Insolvenzverfahren, da der Gesetzgeber offenbar befürchtete, dass der Schuldner Anmeldungen aus schikanösen Motiven widersprechen könnte. ${ }^{527}$ Seine Interessen daran, dass nur berechtigte Insolvenzforderungen am Verfahren teilnehmen, fallen mit dem der übrigen Insolvenzteilnehmer zusammen und werden für alle Beteiligten grundsätzlich vom Insolvenzverwalter wahrgenommen. ${ }^{528}$

Der feststellungshindernde Widerspruch ist also die einzige Möglichkeit für die Insolvenzgläubiger und den Insolvenzverwalter, die angemeldeten Ansprüche der anderen Insolvenzgläubiger von der Teilhabe an der Insolvenzmasse auszuschließen. Darüber hinaus hat der Widerspruch eines Stimmberechtigten oder des Insolvenzverwalters zur Folge, dass dem Widerspruchsbetroffenen ein Stimmrecht zunächst nur noch gem. $\S 77$ II InsO zuteilwerden kann. ${ }^{529}$ Inwieweit sich ein Widerspruch auf die eigene Widerspruchsberechtigung auswirkt, wird noch im Weiteren zu zeigen sein. ${ }^{530}$

\section{a) Widerspruchsberechtigung und Widerspruchspflicht}

Widerspruchsberechtigt sind nebeneinander der Insolvenzverwalter, der Sachwalter (§ 283 I 1 InsO) oder der Treuhänder (§§ 176 S.2, 313 I 1 InsO), die Insolvenzgläubiger und der Schuldner (evtl. als Eigenverwalter, $\S 283$ I InsO). Daher kann auch eine Insolvenzforderung von mehreren Beteiligten gleichzeitig bestritten werden. Wie bereits dargestellt, ${ }^{531}$ sind jedoch nur diejenigen Insolvenzgläubiger widerspruchsberechtigt, die durch ihre ordnungsgemäße Forderungsanmeldung auch formell Verfahrensbeteiligte geworden sind und sich dadurch der gegenseitigen

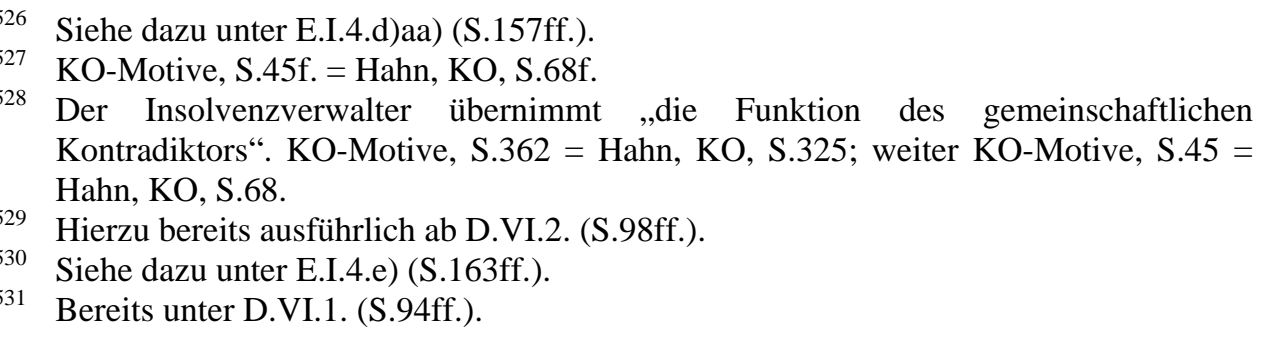


Einflussnahme auf ihre Insolvenzforderungen und Verfahrensrechte unterwerfen.

Im Gegensatz zu den Insolvenzgläubigern, denen es aufgrund ihrer freiwilligen Teilnahme am Verfahren je nach Interesse freigestellt ist, ob sie einer angemeldeten Insolvenzforderung widersprechen oder nicht, ${ }^{532}$ ist der Insolvenzverwalter sowohl im Interesse aller Beteiligten, ${ }^{533}$ insbesondere aber aufgrund der pflichtgemäßen Ausübung und Erfüllung der ihm durch seine Amtsstellung übertragenen Verwaltung der Insolvenzmasse verpflichtet, gegen sämtliche angemeldeten Forderungen Widerspruch zu erheben, die nach seiner materiellrechtlichen Prüfung keine Berechtigung haben dürfen, an dem Insolvenzverfahren und der Verteilung der Insolvenzmasse teilzuhaben. ${ }^{534}$ Sollte er dies nicht tun, kann er sich gem. § 60 I InsO schadenersatzpflichtig machen.

\section{b) Widerspruchszeitpunkt}

Unabhängig von einer wirksamen Erhebung ${ }^{535}$ des Widerspruchs besagt das Gesetz grundsätzlich nicht, dass eine angemeldete Insolvenzforderung allein im Prüfungstermin bestritten werden könnte, weswegen Unstimmigkeiten über die Teilnahmeberechtigung angemeldeter Insolvenzforderungen verfahrenbeschleunigend bereits im Vorfeld des Prüfungstermins einer Klärung zugeführt werden können. Daher werden regelmäßig schon bei der Einsicht in die niedergelegte Tabelle und deren beigefügte Unterlagen die angemeldeten Insolvenzforderungen durch die Beteiligten eingehend auf ihre Teilhabeberechtigung an der Insolvenzmasse geprüft und Widersprüche der Widerspruchsberechtigten schriftlich oder zur Niederschrift der Geschäftsstelle (§ 24 II Nr.3 RPflG; § 129a ZPO i.V.m. § 4 InsO) angebracht und in der vom Urkundsbeamten

532 KO-Motive, S.362 = Hahn, KO, S.325: „Die Theilnahme der einzelnen Gläubiger [am Prüfungstermin]...ist...naturgemäß eine freiwillige; sie können erscheinen und sich erklären, brauchen es aber nicht; ihr Schweigen gilt...als Zustimmung zu den Erklärungen des Verwalters [etwa Erhebung oder Unterlassung eines Widerspruchs].“

533 Dazu unter E.I.2.c) (S.136ff.).

534 Siehe schon E.I.2.c) (S.136ff.); vgl. auch KO-Motive, S.362 = Hahn, KO, S.325; weiter Baur/Stürner, Insolvenzrecht, Rn.5.7, 10.1ff.; MüKo-InsO/Schumacher, § 178 Rn.18 m.w.N. Ausführlich zur Amtsstellung des Insolvenzverwalters etwa Uhlenbruck/Uhlenbruck, § 56 Rn.72.

535 Dazu sogleich unter E.I.4.c)aa) (S.144ff.). 
(und eben nicht vom Insolvenzverwalter weiter) ${ }^{536} \mathrm{zu}$ führenden Tabelle vermerkt. Der Insolvenzverwalter selbst wird meist schon während der Erstellung der Tabelle bei ihm bedenklich erscheinenden Anmeldungen seine Widersprüche vermerkt haben. ${ }^{537}$ Dadurch wird allen Verfahrensbeteiligten, insbesondere aber den widerspruchsbetroffenen Gläubigern ermöglicht, sich so früh und intensiv wie möglich mit den Widersprüchen und deren Abhilfe zu befassen, so dass bis zum Prüfungstermin notwendige Klärungen weitgehend abgeschlossen sind und die Prüfungsergebnisse insoweit feststehen. ${ }^{538}$

Im Prüfungstermin besteht nur die letzte Möglichkeit überhaupt, einen Widerspruch zu erheben, ${ }^{539}$ denn die geprüften und unwidersprochenen Insolvenzforderungen gelten bereits im Prüfungstermin als rechtskräftig festgestellt ( $§ 178$ I, III InsO). ${ }^{540}$ Hierfür spricht auch die eindeutige Regelung des $\S 186$ I 1 InsO, wonach einzig dem Schuldner die Möglichkeit zusteht, durch die Wiedereinsetzung in den vorherigen Stand einen Widerspruch „nachzuholen“, wenn er den Prüfungstermin unverschuldet versäumt hat. Dies ist jedoch nur deshalb möglich, weil sein Widerspruch nicht die Feststellung der Insolvenzforderung und damit den eigentlichen Fortgang des Verfahrens (be-)hindert, sondern „lediglich“ die fehlende Vollstreckbarkeit der bestrittenen Insolvenzforderung nach Beendigung des Verfahrens (§ 201 II 1 InsO) zur Folge hat. ${ }^{541}$

Ein Widerspruch ist im Prüfungstermin daher solange möglich, bis das Insolvenzgericht die Prüfung der Insolvenzforderung im Prüfungstermin für abgeschlossen erklärt. ${ }^{542}$

Siehe bereits unter D.V.2. (S.85ff.).

Siehe auch unter E.III.5.a) (S.222ff.).

38 Siehe bereits unter E.I.3. (S.137ff.).

539 Kübler/Prütting/Bork/Pape, § 176 Rn.6; Uhlenbruck/Uhlenbruck, § 176 Rn.13; vgl. auch Begr. zu § 203 (§ 176 InsO) RegE, BT-Drucks. 12 /2443, S.184.

540 Siehe unter E.III.1. (S.207f.).

541 Entsprechend in $\S 165 \mathrm{KO}$ geregelt gewesen.

542 Haarmeyer/Wutzke/Förster, InsO, § 7 Rn.55; 


\section{c) Form und Inhalt des Widerspruchs}

\section{aa) Form des Widerspruchs}

Schon aus dem Wortlaut des § 176 S.1 und 2 InsO („Im Prüfungstermin... Die Forderungen, die... bestritten werden") $)^{543}$ ergibt sich, dass der Widerspruch als Prozesshandlung ${ }^{544}$ für seine Wirksamkeit grundsätzlich nur mündlich im Prüfungstermin erhoben werden kann. ${ }^{545}$ Soweit also üblicherweise Widersprüche gegen eine Insolvenzforderung bereits im Vorfeld lediglich schriftlich oder zur Niederschrift erhoben wurden, ist zu ihrer Wirksamkeit die zusätzliche bzw. erneute mündliche Erhebung/Bekräftigung im Prüfungstermin notwendig. ${ }^{546}$ Das Insolvenzgericht hat daher gesondert im Prüfungstermin die Beteiligten zum Bestreiten der angemeldeten Insolvenzforderungen aufzufordern ${ }^{547}$ und darauf hinzuweisen, dass ein Nichtbestreiten im Termin die Feststellung der Insolvenzforderung nach § 178 I InsO zur Folge hat. Die Zulassung des lediglich schriftlichen oder im Vorfeld zur Niederschrift angebrachten Widerspruchs würde die grundsätzliche Mündlichkeit des Prüfungstermins (vgl. § 5 III InsO) und vor allem die Intention des § 176 S.2 InsO - die Erörterung des Widerspruchs und die dadurch gegebene Möglichkeit einer Aufklärung des Streitpunktes - unterlaufen. Sollten die widerspruchsberechtigten Verfahrensbeteiligten, die lediglich vor dem Prüfungstermin schriftlich oder zur Niederschrift Widersprüche erhobenen hatten, daher ihren Widerspruch im Prüfungstermin nicht mündlich bekräftigen, entfaltet ihr Widerspruch grundsätzlich keine Wirkung. Entsprechend ist die Tabelle vom protokollierenden Insolvenzgericht zu

543 Darüber hinaus auch aus dem Wortlaut des § 178 I 1 InsO: „Eine Forderung gilt als festgestellt, soweit gegen sie im Prüfungstermin ... ein Widerspruch weder vom Insolvenzverwalter noch von einem Insolvenzgläubiger erhoben wird...“

544 Der Widerspruch ist Prozesshandlung, da er auf das Verfahren und die Insolvenzgläubigerrechte durch seine feststellungs- (§ 178 I 1 InsO) bzw. vollstreckungshindernde ( $§ 201$ II InsO) Wirkung unmittelbar einwirkt. Es gelten daher die allgemeinen Voraussetzungen jeder Prozesshandlung. - Gottwald/Eickmann, § 64 Rn.7; Uhlenbruck/Uhlenbruck, § 176 Rn.7; GrafSchlicker/Graf-Schlicker, § 176 Rn.13.

545 Ganz h.M. RGZ 57, 270 (274); HK/Irschlinger, § 176 Rn.5; Braun/Specovius, § 176 Rn.12; Kübler/Prütting/Bork/Pape, § 176 Rn.6; KS/Eckardt, S.578 Rn.26; Uhlenbruck/Uhlenbruck, § 176 Rn.11; Graf-Schlicker/Graf-Schlicker, § 176 Rn.13; MüKo-InsO/Nowak, § 176 Rn.29 m.w.N.

546 A.A. Nerlich/Römermann/Becker, § 176 Rn.21.

547 Vgl. Begr. zu $\S 203$ (§ 176 InsO) RegE, BT-Drucks. 12/2443, S.184; Braun/Specovius, § 176 Rn.12. 
berichtigen (§ 178 II 1 InsO). Ein schriftlicher Widerspruch ist allein bei Forderungsprüfungen im schriftlichen Verfahren ( $\S \S 5$ II 1, 177 I 2, II InsO) wirksam. ${ }^{548}$ Diese Regelung wurde erst nachträglich in die Insolvenzordnung aufgenommen ${ }^{549}$ und stellt damit die einzige Ausnahme zur grundsätzlichen Mündlichkeit des Verfahrens dar. Die im Vorfeld des Prüfungstermins angebrachten Widersprüche sind daher zunächst Ankündigungen und dienen den (übrigen) Verfahrensbeteiligten verfahrenstechnisch lediglich zur Ermittlung und Orientierung der wohl zu erwartenden Ergebnisse des Prüfungstermins. Wie bereits erörtert, ${ }^{550}$ wird den Widerspruchsbetroffenen dadurch die Möglichkeit eröffnet frühzeitig auf die zu erwartende Verfahrenssituation zu reagieren und noch vor dem eigentlichen Prüfungstermin den Gründen des Widerspruchs nachzugehen und diese nach Möglichkeit auszuräumen, um so dem letztlich nur im Prüfungstermin wirksam erhobenen Widerspruch zuvorzukommen, oder auch ihre Insolvenzforderung vom Verfahren zurückzuziehen. Insofern dienen die bereits im Vorfeld erhobenen, aber bis dahin unwirksamen Widersprüche der Beschleunigung des Insolvenzverfahrens, insbesondere im Hinblick auf den Prüfungstermin und der dortigen Erörterung der von einem Widerspruch betroffenen Insolvenzforderungen. ${ }^{551}$ Diese im Vorfeld eröffnete Vorgehensweise bekräftigt die alleinige Anknüpfung des wirksamen Widerspruchs an seine (erneute) Erhebung oder Unterlassung im Prüfungstermin; gewissermaßen als Bestätigung des endgültigen Prüfungsergebnisses, gleich in welche Richtung.

\section{bb) Inhalt des Widerspruchs}

Welchen Inhalts, also in welchem Umfang bzw. in welche Richtung ein Widerspruch von wem erhoben werden kann, ist nicht nur hinsichtlich der übrigen (Teil-) Feststellung der widerspruchsbetroffenen Insolvenz-

\footnotetext{
548 Graf-Schlicker/Graf-Schlicker, § 176 Rn.13; MüKo-InsO/Nowak, § 176 Rn.29; Uhlenbruck/Uhlenbruck, § 178 Rn.7. Siehe auch unter E.II.5. (S.200ff.).

549 Siehe $\S \S$ 205, 206 (§§ 177, 178 InsO) RegE, BT-Drucks. 12/7302, S.76 und zur Begründung S.178f.

550 Siehe bereits unter E.I.4.b) (S.142ff.).

551 Siehe bereits unter E.I.3. (S.137ff.).
} 
forderung und ihrer verfahrensrechtlichen Wirkung, ${ }^{552}$ sondern auch für den nachfolgend zu erörternden ${ }^{553}$ „isolierten Widerspruch“ gegen das Forderungsattribut der vorsätzlich begangenen unerlaubten Handlung von Bedeutung.

Aus dem Wortlaut des $\S 181$ InsO („Die Feststellung kann nach Grund, Betrag und Rang der Forderung... begehrt werden“) in Verbindung mit dem Wortlaut des $\S 178$ II 1 InsO (,... inwieweit die Forderung... festgestellt ist“) ist zu schließen, dass sich der Widerspruch gegen die angemeldete Insolvenzforderung insgesamt, also den Forderungsgrund bzw. deren Verfolgbarkeit im Insolvenzverfahren, oder auch nur gegen bestimmte Eigenschaften (z.B. Höhe oder Rang der angemeldeten Forderung) richten kann. Der Schuldner ${ }^{554}$ hingegen kann nach Sinn und Zweck eines gläubigerautonomen Insolvenzverfahrens gem. §§ 178 I 2, 201 II InsO nur den Grund, die Höhe oder Durchsetzbarkeit der Insolvenzforderung bestreiten, nicht aber den beanspruchten Rang (§§ 38, 39 InsO) oder die Verfolgbarkeit der Forderung im Insolvenzverfahren ( $\S \S 38$, 53ff. InsO). Rang und Verfolgbarkeit der (Insolvenz-)Forderung sind allein für die Haftung des Schuldners mit der Insolvenzmasse von Bedeutung, nicht aber für seine persönliche Haftung nach Beendigung des Insolvenzverfahrens. ${ }^{555}$ Ein Widerspruch gegen diese Forderungseigenschaften kann aufgrund der entsprechenden Zuweisung der Insolvenzmasse ( $§ 38 \mathrm{InsO}$ ) nur von den Insolvenzgläubigern und dem Insolvenzverwalter, der in diesen Fällen die Interessen des Schuldners vertritt, ${ }^{556}$ erhoben werden. Ansonsten könnte der Schuldner durch seinen Widerspruch die Feststellung der Insolvenzforderung nachträglich faktisch doch in Frage stellen bzw. beeinflussen, dies im Gegensatz zu den Insolvenzgläubigern und dem Insolvenzverwalter zwar nicht unmittelbar feststellungshindernd im Prüfungstermin, wohl aber im Rahmen der Erhebung der Feststellungsklage ( $\S \S 184,181$ InsO). Dies würde jedoch den Verfahrensgrundsatz der Gläubigerautonomie unterlaufen, weshalb ein

Dazu ausführlich unter E.III. (S.206ff.).

Nachfolgend unter E.I.4.c)cc) (S.148ff.).

Anders der Schuldner in Eigenverwaltung. Siehe dazu unter E.I.4.d)aa) (S.157ff.).

MüKo-InsO/Schumacher, § 178 Rn.25; KS/Eckardt, S.757 Rn.28; HHK/Herchen, $\S 184$ Rn.13a; Kübler/Prütting/Bork/Pape, § 184 Rn.7.

556 Siehe bereits unter E.I.2.c) (S.136ff.) aber auch differenzierend unter E.I.4.c)cc) (S.148ff.). 
Widerspruch des Schuldners gegen den Rang und die Verfolgbarkeit der Insolvenzforderung abzulehnen ist. ${ }^{57}$

Der Widersprechende hat wegen der Verschiedenheit der weiteren Behandlung des Anspruchs ${ }^{558}$ und hinsichtlich der Verfolgung des Widerspruchs im Rahmen der Feststellungsklage (vgl. § 181 InsO) im Prüfungstermin genau anzugeben, wogegen sich sein Widerspruch richtet. ${ }^{559}$ Die Widerspruchsrichtung stellt somit einen wesentlichen Teil des in der Tabelle als Ergebnis der Prüfungsverhandlungen zu vermerkenden Prüfungsergebnisses dar, ${ }^{560}$ damit auch spätere Änderungen der Einträge aufgrund der Rücknahme eines Widerspruchs oder aufgrund durchgeführter Prozesse zutreffend vorgenommen werden können. So ist der Widersprechende und der Umfang des Widerspruchs bzw. die teilweise Feststellung der betreffenden Insolvenzforderung durch das Insolvenzgericht in die Tabelle einzutragen (§ 178 II 1 InsO), da der beglaubigte Auszug der Tabelle und deren Eintragungen als Grundlage zur eventuellen Beseitigung des Widerspruchs im Feststellungsprozess ( $\S \S 179$ III, 180; 184 II 3 InsO) dient. Eine ausdrückliche Begründung neben der grundsätzlichen (kurzen) Erörterung des Widerspruchs sieht das Gesetz nicht vor. Einer Begründung bedarf es daher nicht. ${ }^{561}$ Die Begründung des Widerspruchs ist vielmehr eine Frage des folgenden Feststellungsprozesses gem. §§ 179ff. InsO. ${ }^{562}$

557 Gleichwohl stehen dem Schuldner Rechtsbehelfe gegen eine Vollstreckung aus der Insolvenztabelle zu. Siehe dazu unter E.III.7. (S.234f. ).

558 Siehe unter E.I.4.f)bb) (S.170ff.) und E.III.3. (S.215ff.).

559 Gottwald/Eickmann, § 64 Rn.5, 27; MüKo-InsO/Schumacher, $\S 178$ Rn.34; Uhlenbruck/Uhlenbruck, § $178 \mathrm{Rn} .7$

560 MüKo-InsO/Schumacher, § 178 Rn.35; Uhlenbruck/Uhlenbruck, § 178 Rn.7; Jaeger/Weber, KO, § 141 Rn.9 m.w.N.

561 Uhlenbruck/Uhlenbruck, § 178 Rn.7; MüKo-InsO/Nowak, § 176 Rn.28; GrafSchlicker/Graf-Schlicker, § 176 Rn.12; FK/Kießner, § 176 Rn.10; Eisner, NZI 2003, 480 (481).

562 KO-Motive, S.363 = Hahn, KO, S.326. 


\section{cc) Isolierte Widerspruchsmöglichkeit gegen das Forderungsattribut}

Fraglich im Zusammenhang der Widerspruchsrichtung ist jedoch, ob und vom wem ein isolierter Widerspruch gegen das in der Anmeldung vorgebrachte Forderungsattribut erhoben werden kann, dass die angemeldete Insolvenzforderung auf einer vorsätzlich begangenen unerlaubten Handlung des Schuldners beruhe, um eine nachinsolvenzliche Zwangsvollstreckung gegen den Schuldner persönlich - insbesondere nach erteilter Restschuldbefreiung - auch für diese Insolvenzforderung zu verhindern (vgl. § $302 \mathrm{Nr} .1 \mathrm{InsO})$.

Fraglich ist zunächst, ob ein isolierter Widerspruch gegen das Forderungsattribut überhaupt erhoben werden kann. Wie zuvor dargelegt, kann sich der Widerspruch gegen sämtliche Eigenschaften der angemeldeten Insolvenzforderung im Ganzen oder auch nur in Teilen richten, unter anderem also auch lediglich gegen den angegebenen Grund und die damit verbundene(n) Anspruchsgrundlage(n) der Insolvenzforderung. ${ }^{563}$ Dies bekräftigt die Gesetzesbegründung zu § $174 \mathrm{II}$ InsO, wo klargestellt wird, dass gegen das Forderungsattribut der vorsätzlich begangenen unerlaubten Handlung der isolierte Widerspruch - in ausdrücklicher Anlehnung an den isolierten Vorrechtsstreit nach der Konkursordnung - möglich ist. ${ }^{564}$ Insofern war mit der Einführung der §§ 174 II, 175 II, 302 Nr.1 InsO logischerweise nicht beabsichtigt, dass der Widersprechende gezwungen sein soll, Insolvenzforderungen zu bestreiten, die er eigentlich - jedenfalls auf nicht deliktischer Grundlage - anerkennen muss, weil er ihnen nichts entgegenzusetzen hat. ${ }^{565}$ Daher kann grundsätzlich ein isolierter Widerspruch gegen die Anspruchsgrundlage

563 Dies war schon im Rahmen des Vorrechtsstreits nach § 146 IV KO anerkannt. RGZ 130, 334 (335); BAG NJW 1986, 1898 (1898); LAG Hamm ZIP 1987, 1267 (1269); Hess/Hess, KO, § 146 Rn.4; Kuhn/Uhlenbruck, KO, § 146 Rn.25 m.w.N.

564 Begr. zu § 174 II InsO RegE, BT-Drucks. 14/5680, S.27f. Zum Vorrechtsstreits nach $\S 146$ IV KO siehe etwa: BAG NJW 1986, 1898 (1898); LAG Hamm ZIP 1987, 1267 (1269); Hess/Hess, KO, § 146 Rn.4; FK/Ahrens, § 302 Rn.11 m.w.N.

565 Vgl. auch Kübler/Prütting/Bork/Pape, § 184 Rn.95. 
der vorsätzlich begangenen unerlaubten Handlung (z.B. aus § 823 II BGB i.V.m. § 266a StGB) erhoben werden. ${ }^{566}$ Ist ein isolierter Widerspruch gegen das Forderungsattribut somit grundsätzlich zulässig, wird man zunächst weiterfragen müssen, wogegen sich dieser genau richtet, um ihn von anderen Widerspruchsrichtungen zu unterscheiden und als isoliert gegen das Forderungsattribut ansehen zu können. Dies wird im weiteren Verlauf im Zusammenhang mit der unterschiedlichen Zweckverfolgung von Widersprüchen von Bedeutung sein.

Entscheidend für einen als isoliert gegen das Forderungsattribut $\mathrm{zu}$ bewertenden Widerspruch ist grundsätzlich die materiellrechtliche Situation/ Qualität der angemeldeten Forderung. ${ }^{567}$ So ist es einerseits möglich, dass der Lebenssachverhalt (Grund der Forderung) lediglich den Tatbestand einer unerlaubten Handlung ausfüllt, andererseits ist es aber auch möglich, dass für denselben Anspruch mehrere Anspruchsgrundlagen bestehen (Anspruchskonkurrenz). Daher ist hier genau zu differenzieren, gegen welchen Teil der Insolvenzforderung sich der Widerspruch richtet. Als isoliert gegen das Forderungsattribut gerichtet kann man den Widerspruch nämlich nur dann ansehen, wenn er nicht dazu führt, dass der materiellrechtliche Anspruch gänzlich entfällt. In diesem Fall wäre sachlich nämlich kein Unterschied mehr zum allgemeinen Widerspruch, der sich gegen die Insolvenzforderung an sich richtet, zu erkennen, und ein isolierter Widerspruch damit auch terminologisch nicht vorhanden. Ein isolierter Widerspruch ist daher nur in zwei Fallkonstellationen denkbar: Entweder er richtet sich (1) allein gegen die rechtliche Beurteilung der richtigen Tatsachen, oder er richtet sich (2) isoliert gegen die Tatsachen, die die Tatbestandsmerkmale einer unerlaubten Handlung erfüllen, wobei daneben weitere Tatsachen existieren, die wiederum die Tatbestandsmerkmale einer nicht deliktischen Anspruchsgrundlage erfüllen, gleichzeitig aber den identischen Anspruch im Sinne eines

566 H.M.: BGH ZIP 2007, 541 (541); NZI 2006, 536 (537); LG Trier NZI 2006, 243 (244); FK/Ahrens, § 302 Rn.11; HK/Irschlinger, § 178 Rn.5a; Braun/Specovius, $\S 176$ Rn.3; Graf-Schlicker/Graf-Schlicker, § 176 Rn.10; MüKo-InsO/Stephan, § 302 Rn.15; HHK/Preß/Henningsmeier, § 178 Rn.11; Kehe/Meyer/Schmerbach, ZInsO 2002, 660 (662f.); Eisner, NZI 2003, 480 (482); Kahlert, ZInsO 2006, 409 (409).

567 Siehe zum Gegenstand der Feststellung bzw. des Widerspruchs unter E.III.3. (S.215ff.). 
einheitlichen Lebenssachverhaltes betreffen. ${ }^{568}$ Wird hingegen diejenige teilidentische Tatsache bestritten, die für den deliktischen und den nicht deliktischen Rechtsgrund anspruchsbegründend ist, so liegt im Bestreiten dieser Tatsache auch ein Bestreiten des jeweils anderen Rechtsgrundes. Damit würde auch kein isolierter Widerspruch mehr vorliegen, sondern ein Widerspruch, der die angemeldete Forderung vollständig bestreitet.

Nicht eindeutig ist allerdings, wem die Erhebung eines solch isolierten Widerspruchs gegen das Forderungsattribut zusteht. Aus § 178 I InsO ergibt sich, dass sowohl der Insolvenzverwalter und die (formellen) Insolvenzgläubiger ( $§ 178$ I 1 InsO) als auch der Schuldner (§ 178 I 2 InsO) einer Forderungsanmeldung widersprechen können, ohne dass dabei hinsichtlich der Widerspruchsrichtungen unterschieden wird. Entscheidend ist hier jedoch, dass die Widerspruchsmöglichkeit des Insolvenzverwalters und der Insolvenzgläubiger einerseits und des Schuldners andererseits unterschiedliche Zweckrichtungen haben. Während der Widerspruch des Insolvenzverwalters und der Insolvenzgläubiger die Feststellung der angemeldeten Forderung hindert, ist dies beim Widerspruch des Schuldners nicht der Fall (vgl. § 178 I 2 InsO).

Das Widerspruchsrecht des Schuldners soll ihn vor einer weiteren Inanspruchnahme durch seine Gläubiger nach dem Insolvenzverfahren schützen (§§ 201 II, 215 II 2, 257 I 1 InsO). ${ }^{569}$ Ein Insolvenzgläubiger, dessen Forderung im Insolvenzverfahren nicht vollständig befriedigt wird, kann nämlich mit einem vollstreckbaren Auszug aus der Tabelle nach Aufhebung des Insolvenzverfahrens gegen den Schuldner persönlich vorgehen (§ 201 II 1, 3 InsO), falls nicht eine Restschuldbefreiung erteilt wird (§ 201 III InsO). Ausschließlich gegen diese Rechtskraft- und Vollstreckungswirkung des Tabelleneintrags außerhalb des Insolvenzverfahrens wendet sich der vom Schuldner erklärte Widerspruch. 570

Das Widerspruchsrecht des Insolvenzverwalters hingegen soll den Schuldner (und insoweit auch die Insolvenzgläubiger hinsichtlich der

568 Näher Eisner, NZI 2003, 480 (483); auch Schmidt, ZInsO 2006, 523 (523).

569 Zum besonderen Fall der Eigenverwaltung durch den Schuldner siehe unter E.I.4.d.)aa) (S.157ff.).

570 LG Trier NZI 2006, 243 (244); MüKo-InsO/Schumacher, § 178 Rn.23ff.; Eisner, NZI 2003, 480 (481); Kehe/Meyer/Schmerbach, ZInsO 2002, 660 (662f.); Schmidt, ZInsO 2006, 523 (523f.). 
Quote) davor schützen, dass keine unberechtigten Forderungen festgestellt und tituliert werden und diese dann auch nicht an einer späteren Verteilung teilnehmen können, so dass der Schuldner keiner (unberechtigt) höheren Nachhaftung - unbeschadet einer möglichen Restschuldbefreiung ausgesetzt ist. Daher ist der Insolvenzverwalter selbstverständlich verpflichtet, die Berechtigung einer angemeldeten Insolvenzforderung zu prüfen und - soweit er diese Berechtigung verneint - der Forderung zu widersprechen. Stellt er bei seiner Prüfung jedoch fest, dass die Insolvenzforderung zumindest ihrem Grund nach berechtigt ist am Verfahren teilzunehmen, darf er der Insolvenzforderung insoweit im Prüfungstermin nicht widersprechen. Dies darf er aber schon dann nicht, wenn die Insolvenzforderung auf mehrere Anspruchsgrundlagen gestützt wird und bereits die Voraussetzungen einer der in Betracht kommenden Anspruchsgrundlagen $\mathrm{zu}$ bejahen sind, da die Insolvenzforderung dann - unabhängig vom Bestehen oder nicht Bestehen der anderen Anspruchsgrundlagen - grundsätzlich zur Teilnahme an der Verteilung der Insolvenzmasse berechtigt ist. Daher bedarf es keiner weiteren Prüfung der übrigen Anspruchsgrundlagen, da diese keinen entscheidenden Einfluss mehr auf seine Widerspruchsentscheidung haben können. Der Insolvenzverwalter hat somit lediglich zu prüfen, ob überhaupt eine Forderungsberechtigung der angemeldeten Forderung besteht, und wenn ja, ob diese auch am Insolvenzverfahren teilnehmen darf oder nicht. Kommt er daher zum Ergebnis, dass die angemeldete Insolvenzforderung als solche - als „Vermögensanspruch“ i.S.d. § 38 InsO, d.h. dem „Betrag“ nach i.S.v. § 174 II InsO - berechtigt ist, hat der Insolvenzverwalter weitere Anspruchsgrundlagen nicht mehr zu prüfen und/oder isoliert gegen den zusätzlichen angemeldeten Schuldgrund der vorsätzlich begangenen unerlaubten Handlung Widerspruch einzulegen. Dies würde hinsichtlich seiner Pflicht zur bestmöglichen Verwaltung und Erhaltung der Insolvenzmasse außerdem zu unnötigen Feststellungsprozessen (§§ 179ff. InsO) führen, die dann eventuell sogar nachteilig, nämlich beim Unterliegen des Insolvenzverwalters, zur Verminderung der Insolvenzmasse durch die entstehenden Prozesskosten (§ 91 I ZPO i.V.m. § 55 I Nr.1 InsO) $)^{571}$ führen würden. Daher könnte sich der Insolvenz-

\footnotetext{
571 Kübler/Prütting/Bork/Pape, § 183 Rn.6; Uhlenbruck/Uhlenbruck, § 184 Rn.6 m.w.N.
} 
verwalter unter Umständen gar selbst wegen aussichtsloser Prozessführung schadenersatzpflichtig gegenüber der Insolvenzmasse machen (§§ $60 \mathrm{I}$, 92 S.2 InsO). ${ }^{572}$ Zudem ergibt sich aus den $\S \S 85,86$ InsO, dass der Insolvenzverwalter nur verpflichtet sein kann, Prozesse für und gegen die Insolvenzmasse aufzunehmen oder neue Prozesse anzustrengen, wenn für den Fall des Obsiegens eine Anreicherung der Masse zu erwarten ist. Der isolierte Widerspruch gegen den Schuldgrund der vorsätzlich begangenen unerlaubten Handlung von Seiten des Insolvenzverwalters und dessen weitere Verfolgung ist für die Insolvenzmasse aber gerade unerheblich, da die Insolvenzforderung an sich trotz des Widerspruchs des Insolvenzverwalters gegen das Forderungsattribut als unbestritten gilt. Ausgehend von den beiden bereits angesprochenen Widerspruchsrichtungen, die überhaupt nur einen isolierten Widerspruch gegen das Forderungsattribut darstellen, wird bei einem isolierten Widerspruch des Insolvenzverwalters gegen das Forderungsattribut die Insolvenzforderung als solche daher stets festgestellt werden, wodurch dessen Inhaber so oder so einen Anspruch auf Teilhabe an der Insolvenzmasse hätte. ${ }^{573}$ Hieraus wird deutlich, dass ein isolierter Widerspruch gegen den zusätzlichen Schuldgrund der vorsätzlich begangenen unerlaubten Handlung nicht in den Aufgabenkreis (Zweckrichtung des Widerspruchs) des Insolvenzverwalters fallen kann, da er grundsätzlich masseneutral ist, ${ }^{574}$ sondern allein die Rechte des Schuldners nach dem Insolvenzverfahren wahrt und auf das Insolvenzverfahren als solches grundsätzlich keinen Einfluss nimmt. ${ }^{575}$ Insofern bezieht sich auch die Pflicht des Insolvenzverwalters gegenüber dem Schuldner, die Insolvenzmasse bestmöglich $\mathrm{zu}$ verwalten und $\mathrm{zu}$ verwerten, um eine möglichst hohe Enthaftung und damit - (lediglich) daraus folgend - eine niedrige Nachhaftung des Schuldners zu erzielen, nur auf die Prüfung, ob der Inhaber der angemeldeten Forderungen im Insolvenzverfahren zur Teilhabe an der Insolvenzmasse überhaupt berechtigt ist und wenn ja, mit

\footnotetext{
572 Auf diesen haftungsrechtlichen Aspekt geht Schmidt, NZI 2006, 523 (524ff.) in seiner Stellungnahme zu der vorliegenden Problematik überhaupt nicht ein. - Vgl. hierzu etwa BGH ZIP 2001, 1376 (1376); OLG Karlsruhe ZIP 1989, 1071 (1071); Uhlenbruck/Uhlenbruck, § 60 Rn.60ff. m.w.N.

573 Auch wenn $\S 201$ InsO an das Ergebnis im Prüfungstermin anknüpft. Dazu auch unter E.III.1 (S.207f.).

574 Brückl, ZInsO 2005, 16 (18).

575 So auch LG Trier NZI 2006, 243 (244); Eisner, NZI 2003, 480 (485).
} 
welchem Betrag. Nicht aber besteht die Pflicht dahin, ob und inwieweit auch noch nach erfolgter Verteilung der Insolvenzmasse, Aufhebung des Insolvenzverfahrens und womöglich noch nach erteilter Restschuldbefreiung die einzelne Forderung gegen den Schuldner aufgrund einer besonderen Anspruchsgrundlage persönlich vollstreckt werden dürfte. Die Prüfung des Insolvenzverwalters bezieht sich nur auf die Rechte der Insolvenzgläubiger und des Schuldner hinsichtlich der Insolvenzmasse im Insolvenzverfahren und gerade nicht darüber hinaus auch (vorgreifend) auf Rechte gegen den Schuldner persönlich nach Beendigung des Insolvenzverfahrens. ${ }^{576}$ Dies spiegelt sich auch in den unterschiedlichen Wirkungen der Widersprüche wieder, wonach dem Widerspruch des Insolvenzverwalters/ Insolvenzgläubigers über seine feststellungshindernde Wirkung hinaus eben nicht auch eine vollstreckungshindernde Wirkung zukommt, wie sie dem Schuldner und dessen persönlichen Interessen vorbehalten ist. Daher ist es auch nicht Aufgabe des Insolvenzverwalters, sondern nur des Schuldners selbst, seine Rechtsposition nach Abschluss des Insolvenzverfahrens zu beeinflussen und dem Forderungsattribut zu widersprechen. ${ }^{577}$

Für diese Ansicht spricht auch die frühzeitige und umfangreiche Hinweispflicht des Insolvenzgerichts gegenüber dem Schuldner, die der Gesetzgeber in § 175 II InsO normiert hat. ${ }^{578}$ Hätte der Insolvenzverwalter das Recht und die Pflicht, dem vom Insolvenzgläubiger behaupteten Schuldgrund der vorsätzlich begangenen unerlaubten Handlung zu widersprechen, ${ }^{579}$ wenn die Forderung unstreitig auch aus einem anderen Schuldgrund berechtigt ist, wäre diese Hinweispflicht quasi überflüssig. ${ }^{580}$ Der Schuldner könnte sich dann nämlich an dem Insolvenzverwalter schadlos halten. Zudem widerspräche ein Recht des Insolvenzverwalters

\footnotetext{
Siehe auch unter E.III.2. (S.208ff.).

LG Trier NZI 2006, 243 (244); HK/Irschlinger, §178 Rn. 5a; Graf-Schlicker/GrafSchlicker, § 176 Rn.10; MüKo-InsO/Stephan, § 302 Rn.15; Braun/Specovius, § 178 Rn.3; Kehe/Meyer/Schmerbach, ZInsO 2002, 660 (663); Eisner, NZI 2003, 480 (485); Brückl, ZInsO 2005, 16 (18).

578 Siehe bereits unter D.V.5. (S.90ff.).

579 So Schmidt, NZI 2006, 523 (524ff.).

580 Anmeldungen, Änderungen und Ergänzungen sind grundsätzlich beim Insolvenzverwalter anzubringen, wodurch ihm die gem. § 174 II InsO ausdrücklich vom Anmeldenden darzulegende Tatsache, dass dieser sich auf die Qualifizierung seiner Forderung als Forderung aus vorsätzlich begangener unerlaubter Handlung beruft, ebenso bekannt ist, wie die Rechtsfolge des $\S 302$ Nr.1 InsO, die an deren Feststellung anknüpft.
} 
zum isolierten Widerspruch gegen das Forderungsattribut seiner Stellung als Amtswalter der Interessen aller Beteiligten, ${ }^{581}$ dessen Aufgabenbereich nur so weit reicht wie seine Stellung als Verwalter der Insolvenzmasse. Ein isoliertes Widerspruchsrecht oder sogar eine Pflicht hierzu würde seine neutrale Stellung hingegen unzulässig weit in Richtung des Schuldners verschieben. ${ }^{582}$ Damit würde er sein Amt über den gesetzlich bestimmten Rahmen hinaus zugunsten des Schuldners wahrnehmen, was mit der unabhängigen Stellung des Insolvenzverwalters - insbesondere auch gegenüber den von seinem Widerspruch betroffenen Insolvenzgläubigern jedoch nicht $\mathrm{zu}$ vereinbaren ist, ${ }^{583}$ sondern fast schon einer anwaltlichen Vertretung des Schuldners im Insolvenzverfahren gleichkäme. Auch in dieser Hinsicht steht dem Insolvenzverwalter daher kein isoliertes Widerspruchsrecht gegen das Forderungsattribut zu.

Unabhängig von dem zu versagenden isolierten Widerspruchsrecht des Insolvenzverwalters besteht zumindest im Rahmen der bereits dargestellten $^{584}$ Vorprüfungspflicht des Insolvenzverwalters hinsichtlich der formellen Kriterien des $§ 174$ InsO die Möglichkeit, den Schuldner seitens des Insolvenzverwalters im Rahmen der Insolvenzforderungsanmeldung vor unberechtigten Anmeldungen von Insolvenzforderungen hinsichtlich des Forderungsattributs aus vorsätzlich begangener unerlaubter Handlung zu schützen. Diese Vorprüfung umfasst nämlich alle formellen Kriterien der Anmeldung des § 174 InsO und somit auch diejenigen bezüglich der Angaben des anmeldenden Insolvenzgläubigers hinsichtlich des darzulegenden Sachverhalts, aus dem sich nach Einschätzung des Insolvenzgläubigers ergibt, dass seiner Forderung eine vorsätzlich begangene unerlaubte Handlung des Schuldners zugrunde liegt (§ 174 II 2. Halbsatz InsO). ${ }^{585}$ Im Rahmen der formellen Vorprüfung des Insolvenz-verwalters hinsichtlich dieser Tatsachenangabe kann natürlich keine inhaltliche Schlüssigkeitskontrolle gefordert werden

\footnotetext{
KO-Motive, S.362 = Hahn, KO, S.325.

Brückl, ZInsO 2005, 16 (18).

Auch dies verkennt Schmidt, NZI 2006, 523 (524ff.) in seinen Ausführungen.

Siehe bereits unter D.IV.5.d) (S.65ff.).

585 Etwas anderes wäre schon nicht mit der Pflicht des Insolvenzverwalters zur ordnungsgemäßen Tabellenführung vereinbar. Das ergibt sich aus dem eindeutigen Wortlaut des $\S 175$ I 1 InsO, der hinsichtlich des Umfangs der Tabellenführung und damit auch hinsichtlich des Umfangs der formellen Prüfungspflicht des Insolvenzverwalters auf $\S 174$ II, III InsO verweist. - Vgl. auch Kübler/Prütting/Bork/Pape, § 184 Rn.63ff.
} 
sein, ${ }^{586}$ ob der vorgetragene Sachverhalt den Tatbestand einer vorsätzlich begangenen unerlaubten Handlung erfüllt oder nicht. ${ }^{587}$ Der Insolvenzverwalter ist vielmehr nur verpflichtet, zu kontrollieren, ob der Insolvenzgläubiger überhaupt einen Sachverhalt vorgetragen hat, aufgrund dessen die Feststellung einer vorsätzlich begangenen unerlaubten Handlung in Betracht kommt. ${ }^{588}$ Sollte dies nicht der Fall sein, hat der Insolvenzverwalter - natürlich nach Hinweis gegenüber dem Anmeldenden zur möglichen Ergänzung seiner Anmeldung in diesem Punkt - die (zusätzliche) Aufnahme des Forderungsattributs in die Tabelle abzulehnen. ${ }^{589}$

Fraglich ist jedoch, ob die Versagung des isolierten Widerspruchs auch für die Insolvenzgläubiger zu gelten hat. Die Widerspruchsrichtung der Insolvenzgläubiger ist zwar grundsätzlich die Gleiche wie die des Insolvenzverwalters, jedoch verfolgen die Insolvenzgläubiger dabei - wie auch der Schuldner - hinsichtlich ihrer angemeldeten Insolvenzforderungen ihre eigene persönliche Rechtsposition, die auch über das Insolvenzverfahren hinaus Geltung und Bedeutung hat. Hat der Insolvenzgläubiger selbst eine Insolvenzforderung mit dem zusätzlichen Schuldgrund der vorsätzlich begangenen unerlaubten Handlung angemeldet, konkurriert er nach Abschluss des Insolvenzverfahrens mit ebenfalls privilegierten Insolvenzgläubigern in der Verfolgung seiner festgestellten Ansprüche. Folglich wirken sich die qualifiziert festgestellten Insolvenzforderungen also nicht nur für den Schuldner, sondern auch für die Insolvenzgläubiger mit qualifizierten Insolvenzforderungen untereinander über das Verfahren hinaus aus. Daher werden auch diese Insolvenzgläubiger durch die isolierte Frage der vorsätzlich begangenen unerlaubten Handlung grundsätzlich in ihren Rechten berührt, so dass neben dem Schuldner auch den Insolvenzgläubigern, die selbst eine

586 Siehe bereits unter D.IV.5.a) (S.53ff.).

587 Dies ist gerade Sache des Schuldners/Insolvenzgläubigers im Prüfungstermin bzw. nach erhobenen Widerspruch Sache des Gerichts im Feststellungsprozess gem. $\S \S 179 \mathrm{ff}$. InsO.

588 FK/Ahrens, § 302 Rn. 10c; Kübler/Prütting/Bork/Pape, § 184 Rn.69; auch Henning, ZInsO 2004, 585 (587); zum möglichen Inhalt einer solchen Anmeldung siehe Brückl, ZInsO 2005, 16 (17).

589 Ist die Anmeldung ansonsten formell ordnungsgemäß erfolgt, ist die Insolvenzforderung zumindest insoweit zum Insolvenzverfahren zuzulassen und in die Tabelle aufzunehmen. Zur Problematik der Bezugnahme bei der Anmeldung auf titulierte Forderungen aus vorsätzlich begangener unerlaubter Handlung siehe unter E.I.4.f)bb) (S.170ff.). 
Forderung aus vorsätzlich begangener unerlaubter Handlung angemeldet haben, ein isoliertes Widerspruchsrecht gegen das Forderungsattribut zuzusprechen ist. ${ }^{590}$ Die von diesem isolierten Widerspruch betroffene Insolvenzforderung kann dann insoweit, also nur hinsichtlich ihrer Eigenschaft als Forderung aus vorsätzlich begangener unerlaubter Handlung, nicht zur Tabelle festgestellt werden. ${ }^{591}$

Somit bleibt festzuhalten, dass nur der Schuldner und diejenigen Insolvenzgläubiger, die ebenfalls eine Forderung aus vorsätzlich begangener unerlaubter Handlung zum Verfahren angemeldet haben, dem Forderungsattribut einer Insolvenzforderung isoliert widersprechen können.

\section{d) Wirkung des Widerspruchs}

Hinsichtlich der Wirkung des Widerspruchs ist grundsätzlich zu unterscheiden, von wem er erhoben wird:

Unabhängig vom eigentlichen Gegenstand der Feststellung ${ }^{592}$ gilt die angemeldete untitulierte Insolvenzforderung als festgestellt und damit zur Teilhabe an der Insolvenzmasse berechtigt, soweit gegen sie im Prüfungstermin oder schriftlichen Verfahren (§§ 5 II 1, 177 I 2, II InsO) ein Widerspruch weder vom Insolvenzverwalter, Sachwalter, Treuhänder oder auch vom Schuldner als Eigenverwalter noch von einem Insolvenzgläubiger erhoben wird, oder soweit ein erhobener Widerspruch beseitigt ist (vgl. $\S 178$ I 1 InsO). Angemeldete titulierte Insolvenzforderungen werden bei einem Widerspruch zwar ebenfalls nicht festgestellt, nehmen jedoch gleichwohl an der Verteilung teil (arg. § 189 I InsO).

Ein Widerspruch des Schuldners steht der Feststellung der Insolvenzforderung dagegen grundsätzlich nicht entgegen (§ 178 I 2 InsO). Er hindert jedoch gem. § 201 II InsO die spätere Vollstreckbarkeit der festgestellten Insolvenzforderung nach Verfahrensbeendigung.

\footnotetext{
590 Kehe/Meyer/Schmerbach, ZInsO 2002, 660 (664); wohl auch Mäusezahl, ZInsO 2002, 462 (468); FK/Kießner, § 174 Rn.27.

591 Zur generellen Möglichkeit der Teilfeststellung siehe unter E.III.1. (S.207ff.).

592 Siehe unter E.III.3. (S.215ff.).
} 


\section{aa) Eigenverwaltung des Schuldners}

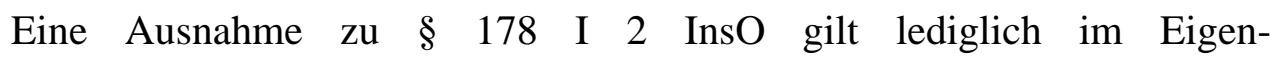
verwaltungsverfahren, in dem neben dem Widerspruch des Sachwalters $^{593}$ und der Insolvenzgläubiger auch dem des Schuldners als Eigenverwalter eine feststellungshindernde Wirkung zukommt (§ 283 I 2 InsO). Das Widerspruchsrecht des Schuldners folgt in diesem Fall bereits aus der (fort-)bestehenden Verwaltungs- und Verfügungsbefugnis (§ 270 I 1 InsO) über sein Vermögen, ${ }^{594}$ wodurch er grundsätzlich im gleichen Umfang mit den entsprechenden Rechten und Pflichten des (eigentlichen) Insolvenzverwalters verwalten und verfügen kann. ${ }^{595}$ Soweit die Vorschriften über die Eigenverwaltung nichts anderes bestimmen, gelten für den Schuldner als Eigenverwalter und den Sachwalter die allgemeinen Vorschriften der Insolvenzordnung (§ 270 I 2 InsO) und damit auch die bisher gemachten Ausführungen zur Forderungsanmeldung - die jedoch ausschließlich beim Sachwalter zu erfolgen hat (§ 270 III 2 InsO) $)^{596}$ - und deren Prüfung im Prüfungstermin. ${ }^{597}$

Fraglich im Rahmen eines Widerspruchs des Eigenverwalters ist jedoch, ob ihm zugestanden werden muss, dass er seinen Widerspruch aufgespaltet ausüben kann, also einerseits feststellungshindernd als Amtswalter der Insolvenzmasse (§§ 178 I 1, 283 I InsO) und andererseits lediglich vollstreckungshindernd mit dem Widerspruchsrecht als Schuldner (§§ 178

593 Für den Sachwalter ergibt sich sein Widerspruchsrecht aus seiner Rechtsstellung als Beschützer der Gläubigerinteressen (vgl. Begr. zu § 331 (§ 270 InsO) RegE, BTDrucks. 12/2443, S.223). Ihm obliegt es neben den mit der Tabelle zusammenhängenden Aufgaben (§ 270 III 2 InsO) vor allem die Geschäftsführung des Schuldners zu überwachen (§ 274 II 1 InsO) und das vom Schuldner aufzustellende Verteilungsverzeichnis zu prüfen (§ 283 II 2 InsO). Uhlenbruck/Uhlenbruck, § 283 Rn.5; Braun/Riggert, § 283 Rn.1; HK/Landfermann, $\S 270$ Rn.19.

594 Zudem führt er die laufenden Geschäfte (vgl. § 274 II 1 InsO), gestaltet (£ 279 InsO) und realisiert ( $\S 282 \mathrm{InsO}$ ) Gläubigerrechte und übernimmt die Befriedigung der Insolvenzgläubiger ( $\$ 283$ II InsO).

595 MüKo-InsO/Wittig, $§ 283$ Rn.8; Graf-Schlicker/Graf-Schlicker, $§ \quad 283$ Rn.4; HK/Landfermann, § 270 Rn.19; näher Häsemeyer, InsO, Rn.8.13ff.

596 Auch der Sachwalter „hat alle Gesichtspunkte zu erwägen, aus denen sich Bedenken gegen eine Forderung ergeben können.“ Rechtsausschuss zu $§ \quad 344$ (§ 283 InsO), BT-Drucks. 12/7302, S.186.

597 „Außerhalb des Bereichs der Verwaltungs- und Verfügungsbefugnisse gelten für das Insolvenzverfahren, bei dem die Eigenverwaltung... angeordnet ist, die gleichen Bestimmungen wie für ein Insolvenzverfahren mit Insolvenzverwalter.“ Begr. zu $\S 331$ (§ 270 InsO) RegE, BT-Drucks. 12/2443, S.223. 
I 2, 201 II 1 InsO), das er nur für sich persönlich und insbesondere im Hinblick auf $\S 302$ Nr.1. InsO und seine Nachhaftung bei erfolgter Restschuldbefreiung wahrnimmt. ${ }^{598}$

Aus der Stellung des Eigenverwalters und der eindeutigen bzw. gerade undifferenzierten Regelung des § 283 I 2 InsO ergibt sich generell, dass der Eigenverwalter entsprechend dem eigentlichen Insolvenzverwalter ${ }^{599}$ sein Widerspruchsrecht nicht unterschiedlich ausüben kann. Jedoch muss hinsichtlich eines isolierten Widerspruchs gegen das Forderungsattribut der vorsätzlich begangenen unerlaubten Handlung und hinsichtlich seiner Nachhaftung als Schuldner nach einem eventuell durchgeführten Restschuldbefreiungsverfahren gem. § 302 Nr.1 InsO eine Ausnahme gelten. Die Versagung eines Widerspruchsrechts hinsichtlich dieses Forderungsattributs ist im Vergleich zum Insolvenzverwalter für den Schuldner im Rahmen seiner Amtswalterstellung im Eigenverwaltungsverfahren nämlich nicht hinnehmbar, da der Schuldner gleichzeitig auch seine eigenen Interessen als Schuldner mitverfolgt. Hätte der Schuldner im Eigenverwaltungsverfahren kein Recht zum persönlichen isolierten Widerspruch gegen den Schuldgrund der vorsätzlich begangenen unerlaubten Handlung, so könnte dieser für den Schuldner hinsichtlich seiner Haftung nach dem Restschuldbefreiungsverfahren wichtigste Widerspruch im Insolvenzverfahren überhaupt nicht geltend gemacht werden. Als Eigenverwalter besteht für ihn grundsätzlich Anwesenheitspflicht im Prüfungstermin, ${ }^{600}$ so dass bei einer jederzeit möglichen Aufhebung der Eigenverwaltung (vgl. §§ 272, 274 III InsO) dem Schuldner auch keine Widereinsetzung in den vorigen Stand gem. $\S 186$ I InsO zugebilligt werden kann, ${ }^{601}$ um zumindest „nachträglich“ die angemeldeten Insolvenzforderungen aus vorsätzlich begangener unerlaubter Handlung bestreiten zu können. Würde man ihm einen persönlichen isolierten Widerspruch daher nicht zubilligen, wäre er ohne jegliche Widerspruchsmöglichkeit gegen das Forderungsattribut der

598 Bejahend Häsemeyer, InsO, Rn.8.16; MüKo-InsO/Schumacher, § 178 Rn.29f.; BK/Blersch, § 283 Rn.3; ablehnend MüKo-InsO/Wittig, § 283 Rn.11; GrafSchlicker/Graf-Schlicker, § 283 Rn.5; HK/Landfermann, § 283 Rn.5; KS/Pape, 922 Rn.50; FK/Foltis, § 283 Rn.2.

599 Hinsichtlich des abzulehnenden isolierten Widerspruchsrechts gegen das Forderungsattribut des Insolvenzverwalters siehe bereits unter E.I.4.c)cc) (S.148ff.).

600 Bereits unter E.I.2.c) (S.136f.).

601 Nerlich/Römermann/Becker, § 178 Rn.6. 
Nachhaftung des $\S 302$ Nr.1 InsO ausgesetzt. Fraglich ist jedoch, ob bei Ausübung des Widerspruchs gegen das Forderungsattribut zwischen einem feststellungshindernden und einem vollstreckungshindernden Widerspruch unterschieden werden muss. Dem Eigenverwalter einen allgemeinen vollstreckungshindernden Widerspruch zuzusprechen, ist wegen der eindeutigen Regelungen des § 283 I InsO abzulehnen und auch nicht nötig, da sein feststellungshindernder Widerspruch als Eigenverwalter ein wesentliches Mehr im Vergleich zu seinem persönlichen Widerspruch als Schuldner darstellt. Die Wirkung des Eigenverwalterwiderspruchs erstreckt sich schließlich gerade nicht nur auf Grund, Höhe und Durchsetzbarkeit der angemeldeten Forderung wie beim Widerspruch als (normaler) Schuldner, ${ }^{602}$ sondern darüber hinaus auch auf die Teilnahme(berechtigung) der Forderung am Insolvenzverfahren an sich, so dass die Forderung teilweise (z.B. hinsichtlich ihrer Eigenschaft des Forderungsattributs) oder auch insgesamt schon gar nicht festgestellt wird und sich der persönliche vollstreckungshindernde Widerspruch als Schuldner erübrigt.

Damit bleibt festzuhalten, dass dem Schuldner in Eigenverwaltung im Rahmen seines feststellungshindernden Widerspruchs auch ein Widerspruch gegen das Forderungsattribut der vorsätzlich begangenen unerlaubten Handlung zusteht. Insofern geht hier der Umfang des Eigenverwalterwiderspruchs notwendigerweise über den des Insolvenzverwalters und der Insolvenzgläubiger hinaus bzw. deckt sich mit dem der Insolvenzgläubiger von angemeldeten Forderungen aus vorsätzlich begangener unerlaubter Handlung, die - wie bereits festgestellt ${ }^{603}$ - dem Forderungsattribut ebenfalls isoliert widersprechen können.

\section{bb) Das „vorläufige Bestreiten“ des Insolvenzverwalters}

602 Siehe bereits unter E.I.4.c)bb) (S.145ff.).

603 Siehe bereits unter E.I.4.c)cc) (S.148ff.). 
Vor allem in Großverfahren mit zahlreichen Forderungsanmeldungen hat sich schon unter der Konkursordnung in der Praxis des Insolvenzverwalters das „Bestreiten unter Vorbehalt“ herausgebildet. Häufig kann der Insolvenzverwalter wegen der Fülle von angemeldeten Insolvenzforderungen und weit reichenden anderen Aufgaben bis zum Prüfungstermin noch nicht die materielle Berechtigung aller angemeldeten Insolvenzforderungen klären, so dass das „vorläufige Bestreiten“ - wie sich zeigen wird - allein ein Instrument der Zeitgewinnung in der Praxis ist, um seitens des Insolvenzverwalters keinen Anlass für vorzeitige Feststellungsklagen zu bieten.

Das „vorläufige Bestreiten“ hat - obwohl teilweise auch schon unter der Konkursordnung praktiziert ${ }^{604}$ - in der jetzigen Insolvenzordnung keine Berücksichtigung gefunden. Die §§ 176, 179 InsO sehen nicht vor, dass eine angemeldete Insolvenzforderung „nur vorläufig“ bestritten werden kann. ${ }^{605}$ Daher ist auch eine nur „vorläufig bestrittene“ Forderung als wirksam bestritten im Sinne des $\S \S 178$ I, 179 I InsO anzusehen. ${ }^{606}$ Der Zusatz „vorläufig“ kann daran schon nichts ändern, weil letztlich jedes Bestreiten insoweit nicht endgültig ist, als der Widerspruch zurückgenommen werden kann. ${ }^{607}$ Insofern gibt es keine eigenständige Verfahrensweise des „vorläufigen Bestreitens“ durch den Insolvenzverwalter. Die Insolvenzordnung gibt dem Insolvenzgericht und dem Insolvenzverwalter in den Fällen der Großverfahren lediglich die Möglichkeit, die Höchstfristen für die Terminierung des Prüfungstermins auszunutzen, sodass zwischen Eröffnung des Insolvenzverfahrens und Prüfungstermin fünf Monate liegen dürfen (§ 28 I 2 InsO i.V.m. § 29 I Nr.2 InsO). ${ }^{608}$ Können in diesem Prüfungstermin nicht alle angemeldeten Insolvenzforderungen abschließend geprüft werden, besteht weiterhin

604 Siehe etwa BGH InsO 2006, 320 (320) m.w.N.

605 Die Insolvenzordnung hat die Regelung des § 146 I 1 KO ohne inhaltliche Veränderung übernommen (vgl. Begr. zu $§ 207$ (§ 179 InsO) RegE, BT-Drucks. 12/2443, S.185), so dass der Ansicht von BK/Blersch, § 178 Rn.6, dass sich die Zulässigkeit des vorläufigen Bestreitens aus dem „neuen“ Wortlaut des § 179 InsO herleiten lasse, nicht gefolgt werden kann. Zudem spricht das Gesetz lediglich von „bestreiten“.

606 Mittlerweile wohl ganz h.M. BGH InsO 2006, 320 (320); BAG ZIP 1988, 1587 (1589); KS/Eckardt, S.774f. Rn.56; Kübler/Prütting/Bork/Pape, § 179 Rn.6; GrafSchlicker/Graf-Schlicker, § 176 Rn.14f.; HHK/Preß/Henningsmeier, § 178 Rn.9; MüKo-InsO/Schumacher, § 178 Rn.37 m.w.N. E.I.4.f) (S.168ff.).

608 Siehe bereits unter D.II. (S.30ff.) und D.IV.7. (S.72ff.). 
unproblematisch die Möglichkeit, auf Antrag eines Beteiligten ${ }^{609}$ oder von Amts wegen (§§ 136 III, 227 ZPO i.V.m. § 4 InsO) den Prüfungstermin im Termin durch Beschluss des Insolvenzgerichts $\mathrm{zu}$ vertagen. Eine öffentliche Bekanntmachung des weiteren Prüfungstermins ist dann nicht erforderlich (§ 74 II 2 InsO).

Jedoch kann sich der Insolvenzverwalter gleichwohl im Prüfungstermin zu den von ihm bestrittenen Insolvenzforderungen ohne Weiteres erklären und auch die „Vorläufigkeit“ seines Widerspruchs unter der Rubrik „Bemerkungen“ in der Tabelle durch das Insolvenzgericht protokollieren lassen. Dies wird insbesondere dann der Fall sein, wenn dem Insolvenzverwalter trotz bereits erfolgter Prüfung erst durch neue Informationen und Erkenntnisse unmittelbar im Prüfungstermin die eine oder andere angemeldete Insolvenzforderung doch noch als zur Teilnahme am Insolvenzverfahren nicht berechtigt erscheint. Letztlich geht es aber beim Bestreiten einer Insolvenzforderung durch den Insolvenzverwalter unter dem Hinweis, dass diese angemeldete Insolvenzforderung von ihm („vorläufig“) noch nicht oder nicht vollständig geprüft werden konnte, nur um die Frage, ob und wie lange es dem Insolvenzgläubiger einer „vorläufig bestrittenen Insolvenzforderung“ zuzumuten ist, mit der Erhebung der Feststellungsklage gem. $\S \S 179$ I, 180 I InsO oder der Weiterführung des unterbrochenen Rechtsstreits ( $§ 180$ II InsO) zu warten und eine für den Insolvenzverwalter nachteilige Kostenfolge im Rahmen des § 91a ZPO bei einer sofortigen Anerkennung der bestrittenen Insolvenzforderung im Prozess zu vermeiden. Zwar wird auch durch das „vorläufige Bestreiten“ wirksam bestritten, wodurch kein Unterschied hinsichtlich der Verfolgung des Feststellungsinteresses ${ }^{610}$ einer bestrittenen Forderung im Rahmen der $\S \S$ 179ff. InsO entsteht, doch folgt gerade aufgrund des Hinweises „vorläufig“ nicht in jedem Fall, dass der Insolvenzverwalter auch wirklich Anlass für eine Feststellungsklage im Sinne des § 93 ZPO gegeben hat. Diese Frage ist vielmehr unter Würdigung aller Umstände des Einzelfalls nach den zu § 93 ZPO entwickelten Grundsätzen zu beantworten, ${ }^{611}$ die gerade auch im Rahmen der Kostentragung des § 91a ZPO heranzuziehen

609 Siehe nur MüKo-InsO/Nowak, § 176 Rn.13ff. m.w.N.

610 Siehe dazu folgend unter E.I.4.f)bb) (S.170ff.).

611 Siehe etwa bei Musielak/Wolst, ZPO, § 91a Rn.10ff.; Zöller/Herget, ZPO, § 93 Rn.1ff. 
sind. ${ }^{612}$ Auch wenn das „,vorläufige Bestreiten“ im Prüfungstermin keine eigenständige Verfahrensweise des Widerspruchs darstellt, macht der Insolvenzverwalter durch eine solche Erklärung zur Tabelle dennoch verfahrenstechnisch deutlich, dass er die Forderung nur deshalb bestreitet, weil er sich zu ihr noch nicht abschließend erklären kann. Insofern weiß der Insolvenzgläubiger, dass eine Feststellung seiner angemeldeten Insolvenzforderung durch Rücknahme des Widerspruchs seitens des Insolvenzverwalters jederzeit noch möglich ist, mit der Folge, dass sich die zu erhebende Feststellungsklage oder eine Fortsetzung des anhängigen unterbrochenen Rechtsstreits (vgl. § 180 InsO) erübrigen kann und ihm so die Kostenlast zufallen könnte (§ 91a i.V.m. § 93 ZPO). Daher hat sich der betroffene Insolvenzgläubiger beim Insolvenzverwalter zu vergewissern, ob dieser seinen Widerspruch aufrechterhält, bevor er sein Feststellungsinteresse gem. $\S \S 179 f f$. InsO verfolgt, um größtmögliche Sicherheit bezüglich eines - trotz Nachfrage - zu führenden Feststellungsstreits gem. §§ 179ff. InsO und der Kostenfrage nach den $\S \S$ 93, 91a ZPO zu erhalten. Dies dient einer verfahrensökonomischen Erledigung des durch das „vorläufige Bestreiten“ eingetretenen Schwebezustandes und verletzt auch keine anerkennenswerten Interessen des Insolvenzgläubigers, ${ }^{613}$ zeigt aber auch, dass der „,vorläufige Widerspruch“ zumindest mittelbar verfahrenstechnische Auswirkung auf die weitere Vorgehensweise des betroffenen Insolvenzgläubigers im Insolvenzverfahren hat. Der Insolvenzgläubiger kann dem Insolvenzverwalter im Rahmen der Nachfrage eine angemessene Frist zur abschließenden Entscheidung über seinen „vorläufigen Widerspruch“ hinsichtlich der angemeldeten Forderung setzen.

Die Grundsätze des § 93 ZPO können jedoch nur bis zur erstmaligen öffentlichen Bekanntmachung des Verteilungsverzeichnisses (§ 188 InsO) eingreifen, weil sich hieran die gesetzliche Ausschlussfrist von zwei Wochen für den Nachweis der Erhebung der Feststellungsklage anschließt

612 H.M. BGH InsO 2006, 320 (321); OLG München WM 2005, 1859 (1860); OLG Düsseldorf ZIP 1994, 638 (638); Kübler/Prütting/Bork/Pape, § 179 Rn.7; HK/Irschlinger, § 179 Rn.4; Graf-Schlicker/Graf-Schlicker, § 176 Rn.15f.; HHK/Preß/Henningsmeier, § 178 Rn.9; MüKo-InsO/Schumacher, § 178 Rn.37 m.w.N.

613 BGH InsO 2006, 320 (321); Kübler/Prütting/Bork/Pape, § 179 Rn.7. 
(§ 189 I InsO), ${ }^{614}$ und der Insolvenzgläubiger ab dann Feststellungsklage erheben muss, wenn er seine angemeldete Insolvenzforderung bei der anstehenden Verteilung berücksichtigt wissen will (vgl. § 189 III InsO).

\section{e) Verlust des Widerspruchsrechts}

Eine Regelung, wie sie $\S 77$ InsO für das Stimmrecht der Insolvenzgläubiger bestrittener Forderungen enthält, besteht für das Widerspruchsrecht nicht. Daher ist fraglich, woran ein Verlust des Widerspruchsrechts der Insolvenzgläubiger zu knüpfen ist. ${ }^{615}$

\section{aa) Bindung an bestrittene Insolvenzforderung}

Der Verlust des Widerspruchsrecht ist jedenfalls nicht direkt an einen Widerspruch gegen die eigene Insolvenzforderung $\mathrm{zu}$ binden, da es ansonsten zu einem „Wettlauf der Widersprüche“ käme bzw. ein Leichtes wäre, die Insolvenzgläubiger willkürlich von einem ihrer elementaren Verfahrensrechte auszuschließen. Daher hindert die eigene bestrittene Insolvenzforderung den Insolvenzgläubiger nicht, sein Widerspruchsrecht weiterhin auszuüben. ${ }^{616}$

\section{bb) Bindung an Stimmrechtsverlust}

614 BGH InsO 2006, 320 (321); Graf-Schlicker/Graf-Schlicker, § 176 Rn.16.

615 Der Insolvenzverwalter als Verwaltungs- und Verfügungsbefugter der Insolvenzmasse ( $§ 80$ I InsO) und der Schuldner als unmittelbarer Insolvenzbetroffener und nach Aufhebung oder Beendigung des Insolvenzverfahrens wieder Vermögensbefugter ( $\S \S 201$ I, 215 II InsO) können ihr Widerspruchsrecht grundsätzlich nicht verlieren, wobei der Schuldner durch seinen nur feststellungshindernden Widerspruch sowieso keinen Einfluss auf die Verfahrensrechte der Insolvenzgläubiger während des Insolvenzverfahrens haben kann. Anders natürlich als Eigenverwalter, siehe dazu aber bereits unter E.I.4.d)aa) (S.157ff.) und D.VI.2.b) (S.102ff.).

616 KO-Motive, S.362 = Hahn, KO, S.326; weiter MüKo-InsO/Schumacher, § 178 Rn.20; Uhlenbruck/Uhlenbruck, § 176 Rn.15; Kübler/Prütting/Bork/Pape, § 176 Rn.10 m.w.N. 
Es kann jedoch überlegt werden, den Verlust des Widerspruchsrechts an die Versagung des Stimmrechts im Rahmen der Stimmrechtsfestsetzung gem. § 77 InsO zu binden, da es sich dabei ebenfalls um den Ausschluss eines Verfahrensrechts handelt, dessen Anknüpfungspunkt einen Widerspruch gegen die Forderung eines formellen Insolvenzgläubigers voraussetzt. ${ }^{617}$ Dies würde im Verlauf des Insolvenzverfahrens jedoch zu untragbaren Unsicherheiten hinsichtlich des Widerspruchsrechts führen, da der Widerspruch im Sinne des $\S 77$ InsO nicht (immer) mit dem feststellungshindernden Widerspruch des Prüfungstermins gleichgesetzt werden kann, und solange die Insolvenzforderung nicht festgestellt wurde, die Festlegung des Stimmrechts oftmals bereits vor dem eigentlichen Prüfungstermin durchgeführt wird und darüber hinaus jederzeit neu festgesetzt bzw. abgeändert werden kann. ${ }^{618}$ Dies könnte schließlich dazu führen, dass dem Insolvenzgläubiger schon zu Beginn des eigentlichen Prüfungstermins, wo es entscheidend auf das Widerspruchsrecht ankommt, kein Widerspruchsrecht mehr zusteht. Zudem hat eine Stimmrechtsfeststellung selbst durch einen entscheidenden Beschluss des Insolvenzgerichts ( $§ 77$ II 3 InsO) keine Auswirkungen auf die materiellrechtliche Befugnis der bestrittenen Forderung des Insolvenzgläubigers, ${ }^{619}$ so dass sich insoweit keine Rückschlüsse auf die formelle Insolvenzgläubigerstellung bzw. das Widerspruchsrecht ziehen lassen. ${ }^{620}$ Darüber hinaus wäre ein stimmrechtlicher Anknüpfungspunkt für den Verlust des Widerspruchsrechts hinsichtlich formeller nachrangiger Insolvenzgläubiger erst gar nicht gegeben, da diese kein Stimmrecht haben, das ihnen zu- oder abgesprochen werden könnte (§ 77 I 2 InsO). Letztlich zeigt aber schon $\S 77$ InsO selbst, dass die Stimmrechtsgewährung an einen erhobenen oder unterlassenen Widerspruch anknüpft und nicht umgekehrt, so dass das Widerspruchsrecht als das Grundlegendere von beiden ansonsten für die Insolvenzgläubiger gleichbedeutend wichtigen Verfahrensrechte im Insolvenzverfahren

617 Wie das Stimmrecht beruht auch das Widerspruchsrecht auf der formellen Insolvenzgläubigerstellung. Siehe bereits unter D.VI.1. und 2. (S.94ff., 98ff.).

618 Siehe unter E.I.1.a) und b) (S.129ff., 131ff.) und ab D.VI.2. (S.98ff.).

619 Begr. zu $\S 88$ (§ 77 InsO) RegE, BT-Drucks. 12/2443, S.133f. Siehe auch unter D.VI.2.c) (S.105ff.).

620 Die letztendliche Klärung der Feststellung der bestrittenen Insolvenzforderung erfolgt ausschließlich im prozessualen Feststellungsverfahren gem. §§ 179ff. InsO. Siehe bereits unter D.IV.5.a) (S.53ff.) und auch folgend unter E.I.4.f)bb) (S.170ff.). 
angesehen werden kann. Das „Weiterführende“ ist prinzipiell an das „Grundlegende“ anzuknüpfen und nicht umgekehrt. Die Anbindung der Versagung des Widerspruchsrechts an das Stimmrecht kann daher nicht erfolgen.

\section{cc) Bindung an die formelle Insolvenzgläubigerstellung}

So bleibt als Anknüpfungspunkt für den Verlust des Widerspruchsrechts zunächst nur die formelle Insolvenzgläubigerstellung selbst, durch die das Widerspruchsrecht zuvor auch begründet wurde. Folglich ist fraglich, in welchen Fällen der Insolvenzgläubiger nicht mehr als formeller Verfahrensteilnehmer des Insolvenzverfahrens anzusehen ist.

Wie bereits dargelegt, ${ }^{621}$ ist dies grundsätzlich dann der Fall, wenn der Insolvenzgläubiger (alle) seine angemeldete(n) Forderung(en) vor ihrer Feststellung gem. $\S 178$ InsO vom Verfahren zurücknimmt. ${ }^{622}$ Des Weiteren führt die im gerichtlichen Feststellungsverfahren gem. $\S \S$ 179ff. InsO festgestellte fehlende Berechtigung der (aller) angemeldeten Forderung(en) zur Teilnahme am Insolvenzverfahren - weil sie eben keine Insolvenzforderung im Sinne des $\S 38$ InsO ist - zum Ausschluss vom Insolvenzverfahren und damit zum Verlust der formellen Insolvenzgläubigerstellung und sämtlicher Verfahrensrechte. Zudem scheidet der Insolvenzgläubiger auch durch eine vorzeitige Befriedigung seiner Forderung - gleich auf welche Weise - aus dem Insolvenzverfahren aus, da er durch das Erlöschen seiner Forderung keinerlei Ansprüche mehr gegenüber dem Schuldner hat, die noch irgendwelche Interessen zur weiteren Teilnahme am Insolvenzverfahren hinsichtlich Sinn und Zweck des Insolvenzverfahrens rechtfertigen könnten. ${ }^{623}$

Über diese drei Möglichkeiten des kompletten Verlustes der formellen Insolvenzgläubigerstellung hinaus kann aufgrund der Regelungen der $\S \S 189,190$ InsO jedoch auch in Betracht gezogen werden, dass dem

\footnotetext{
621 Siehe bereits unter D.VII. (S.121ff.).

622 Nach Feststellung der Insolvenzforderung ist nur der Verzicht auf ihre weitere Berücksichtigung im Insolvenzverfahren möglich. Die Verfahrensrechte des Insolvenzgläubigers werden jedoch endgültig festgestellt. Siehe auch unter E.IV. (S.236ff.).

623 Siehe dazu auch unter E.I.4.f)bb) (S.170ff.).
} 
Insolvenzgläubiger einer untitulierten und aufgrund Widerspruchs nicht festgestellten Forderung zwar nicht sein Widerspruchsrecht, aber zumindest dessen weitere Ausübung dann abzusprechen ist, wenn er die Verfolgung seines ursprünglichen Interesses auf Teilhabe an der Insolvenzmasse im Verfahren nicht weiterverfolgt bzw. weiterverfolgen kann und seine angemeldete Forderung damit im Insolvenzverfahren nicht mehr berücksichtigungsfähig ist. Im Gegensatz zum Insolvenzgläubiger mit bestrittener, aber titulierter Forderung ist der Insolvenzgläubiger einer bestrittenen, nicht titulierten Forderung nach Ablauf der Ausschlussfrist von der Teilhabe an der Insolvenzmasse gem. § 189 I, III InsO ausgeschlossen, wenn er innerhalb einer zweiwöchigen Ausschlussfrist nicht den beim Insolvenzverwalter anzubringenden Nachweis erbracht hat, dass er gegen den feststellungshindernden Widerspruch Feststellungsklage erhoben oder einen früheren Rechtsstreits wieder aufgenommen hat. ${ }^{624}$ In diesem Fall hat die Forderung des Insolvenzgläubigers mangels Feststellung zumindest keinen Einfluss mehr auf die Insolvenzmasse als zentralen Anknüpfungspunkt für die Insolvenzgläubigerstellung (§ 38 InsO) und die Quote der übrigen Insolvenzgläubiger, ${ }^{625}$ so dass fraglich ist, ob der Forderungsinhaber weiterhin durch ein Widerspruchsrecht Einfluss auf das Verfahren bzw. die Forderungen anderer Insolvenzgläubiger - etwa auf nachträglich angebrachte Anmeldungen im Schlusstermin - haben darf. An dieser Stelle darf jedoch nicht über die sonstigen Interessen, die der Insolvenzgläubiger trotz eines Ausschlusses von der Teilhabe an der Insolvenzmasse gleichwohl im Insolvenzverfahren verfolgt, hinweggegangen werden. Insofern könnte für den Insolvenzgläubiger insbesondere ein Interesse an der (erleichterten) Schaffung eines Vollstreckungstitels gegen den Schuldner für die Zeit nach Beendigung des Insolvenzverfahrens durch Feststellung seiner Insolvenzforderung zur Tabelle (§§ 201 II, 178 III InsO) und damit verbunden auch das Interesse an einer Feststellung der Insolvenzforderung hinsichtlich seines

\footnotetext{
624 Siehe hierzu auch unter E.II.3.b) (S.194ff.).

625 Nach Ablauf der Ausschlussfrist kann zumindest hinsichtlich der Teilnahme an einer Verteilung kein schutzwürdiges Feststellungsinteressen mehr für eine Feststellungsklage gem. $\S \S$ 179ff. InsO bestehen. Siehe dazu unter E.II.3.b) (S.194ff.).
} 
Stimmrechts (§ 77 I 1 InsO) bestehen. ${ }^{626}$ Daher hat der Insolvenzgläubiger zumindest bis zur Aufhebung des Insolvenzverfahrens ein schutzwürdiges insolvenzrechtliches Interesse hinsichtlich der prozessualen Feststellung seiner Insolvenzforderung gem. $\S \S \quad 179 f f$ InsO, ${ }^{627}$ da der Insolvenzgläubiger auch weiterhin mit den übrigen Insolvenzgläubigern - insbesondere mit denen, die noch nachträglich ihre Insolvenzforderungen anmelden oder ergänzen - nicht nur hinsichtlich einer Mitbestimmungsmöglichkeit im Insolvenzverfahren, sondern auch bezüglich einer nachinsolvenzlichen Vollstreckungsmöglichkeit gegen den Schuldner konkurriert. Dies gilt insbesondere hinsichtlich eines Widerspruchs gegen das nachträglich angebrachte Forderungsattribut der vorsätzlich begangenen unerlaubten Handlung, ${ }^{628}$ falls es darüber hinaus zu einer Restschuldbefreiung des Schuldners kommen sollte (§ 302 Nr.1 InsO) ${ }^{629}$ Daher ist dem Insolvenzgläubiger einer bestrittenen und untitulierten Forderung, der trotz $\S 189$ I, III InsO mit seiner Forderung bei den Verteilungen nicht mehr berücksichtigungsfähig ist, die Ausübung seines Widerspruchsrechts nicht abzusprechen.

Folglich bleibt festzuhalten, dass der Verlust des Widerspruchsrechts allein an den Verlust der formellen Insolvenzgläubigerstellung zu binden ist.

\section{f) Rücknahme und Beseitigung des Widerspruchs}

626 Siehe dazu unter E.II.3.a) (S.192ff.). Auch wenn sich die Stimmrechtfrage zunächst grundsätzlich nach dem Verfahren des $\S 77$ II InsO richtet, so ist es doch nur ein „vorläufiges“ Verfahren, das die Bedeutung des Stimmrechts hinsichtlich eines schutzwürdigen Feststellungsinteresses für den Insolvenzgläubiger durch dessen letztendliche Anknüpfung an eine - wenngleich prozessuale - Feststellung über die Insolvenzforderung ( $§ 77$ I InsO) vielmehr unterstreicht, als dass das Stimmrecht für ein Feststellungsinteresse des Insolvenzgläubigers nicht in Betracht käme.

628 gl. BGH ZIP 1998, 515 (515f.)

Siehe dazu unter D.IV.3.a) (S.44ff.) und weiter unter E.II.3.a) (S.192ff.).

629 Siehe bereits unter E.I.4.c)cc) (S.148ff.). 
Eine Insolvenzforderung gilt im Sinne des § 178 I 1 InsO als festgestellt, wenn ein feststellungshindernder Widerspruch beseitigt worden ist. Der Wortlaut „ein Widerspruch“ ist insoweit missverständlich, als dass anerkanntermaßen alle Widersprüche ${ }^{630}$ für eine Feststellung beseitigt worden sein müssen, ${ }^{631}$ um eine umfassende Klärung der Berechtigung der angemeldeten Forderung bereits im Insolvenzverfahren herbeiführen zu können. Weiterhin ist die festgestellte Insolvenzforderung nach Aufhebung des Insolvenzverfahrens gem. § 201 II 1, 2 InsO auch nur dann gegen den Schuldner persönlich vollstreckbar, wenn ein eventuell seitens des Schuldners erhobener Widerspruch beseitigt worden ist. ${ }^{632}$ Die Beseitigung dieser Widersprüche kann - wie nachfolgend zu zeigen sein wird - nur dadurch erfolgen, dass ihn der Widersprechende zurücknimmt, die Insolvenzforderung im Feststellungsstreit (§£ 179ff. InsO) positiv festgestellt wird oder der Widersprechende sein Widerspruchsrecht - wie vorab aufgezeigt - verliert.

\section{aa) Rücknahme des Widerspruchs}

630 So schon Protokolle zu $\S \S 132,135$ KO (später $\S \S 146,147$ KO), S.92, $175 f=$ Hahn, KO, S.590, 669f.; MüKo-InsO/Schumacher, § 178 Rn.42; KS/Eckardt, S.775f. Rn.58; Kübler/Prütting/Bork/Pape, § 183 Rn.3; Braun/Specovius, § 183 Rn.3; GrafSchlicker/Graf-Schlicker, § 179 Rn.7; HHK/Herchen, § 179 Rn.39; Jaeger/Weber, KO, § 146 Anm.7ff.

631 Dies gilt auch hinsichtlich des Wortlauts von § 183 I InsO, der so verstanden werden muss, dass das Urteil den einzigen, den letzten oder sämtliche Widersprüche verwerfen muss, um die Feststellung der angemeldeten Forderung auszulösen. - Kübler/Prütting/Bork/Pape, § 183 Rn.3; KS-Eckardt, S.776 Rn.58 m.w.N. Gleichwohl ist in den eventuell anhängigen Parallelprozessen gegen die übrigen Widersprüche die Rechtskraftwirkung des Urteils von Amts wegen zu berücksichtigen. - Vgl. Henckel, Parteilehre, S.206ff.

632 Eine teilweise Vollstreckbarkeit bei einer nur teilweise vom Schuldner bestrittenen Insolvenzforderung - auch bei einem lediglich isolierten Widerspruch gegen das Forderungsattribut - ist (entgegen Kübler/Prütting/Bork/Pape, § 174 Rn.46) aufgrund der herbeizuführenden umfassenden Klärung im Insolvenzverfahren abzulehnen. Dies widerspräche auch dem eindeutigen Wortlaut des § 201 II 1 InsO (,... nicht vom Schuldner bestrittenen“), der - im Vergleich zum Wortlaut des § 178 I 1 InsO (... gilt als festgestellt, soweit...") - von einem generellen Widerspruch des Schuldners ausgeht und daher die Annahme einer teilweisen Vollstreckbarkeit eindeutig nicht zulässt. Zudem sieht auch § 201 II 2 InsO für eine Vollstreckbarkeit die grundsätzliche Beseitigung des Schuldnerwiderspruchs vor. 
Die (teilweise) Rücknahme des Widerspruchs ${ }^{633}$ ist grundsätzlich möglich ${ }^{634}$ und als actus contrarius ebenso Prozesshandlung wie dessen Erhebung und hat daher grundsätzlich gegenüber dem Insolvenzgericht zu erfolgen, ${ }^{635}$ welches die Rücknahme des Widerspruchs von Amts wegen in der Tabelle zu vermerken und den anmeldenden Insolvenzgläubiger hinsichtlich seiner Verfolgung im Feststellungsstreit hierüber zu informieren hat. Eine Rücknahme ausschließlich gegenüber dem anmeldenden Insolvenzgläubiger ${ }^{636}$ ist daher abzulehnen. Dies würde ansonsten - im Gegensatz zum Berichtigungsantrag gem. § 183 II InsO durch Urteilsvorlage - nicht nur zu Beweisführungsschwierigkeiten des Anmeldenden hinsichtlich der tatsächlichen Rücknahme durch den ursprünglich Widersprechenden, sondern auch zu Rechtsunsicherheiten für die übrigen Beteiligten führen. Nur die Erklärung der Rücknahme des Widerspruchs zumindest auch gegenüber dem Insolvenzgericht stellt sicher, dass die - falls keine weiteren Widersprüche vorliegen - damit erfolgte Feststellung der nun unwidersprochenen Insolvenzforderung unmittelbar in die Tabelle eingetragen wird und es somit zu keinen Verzögerungen und Rechtsunsicherheiten im Hinblick auf die Richtigkeit der Tabelle ${ }^{637}$ als Grundlage des weiteren Insolvenzverfahrens für die anderen Verfahrensbeteiligten kommt. Dies gilt insbesondere hinsichtlich der Berücksichtigung der Insolvenzforderung durch den Insolvenzverwalter im von ihm zu erstellenden Verteilungsverzeichnis (vgl. § 187 InsO). Die Rücknahme ist dabei mündlich im Prüfungstermin oder danach ${ }^{638}$ bis zur Entscheidung in einem eventuellen Feststellungsprozess schriftlich oder zu Protokoll der Geschäftsstelle (§ 4 InsO i.V.m. § 496 ZPO) möglich, da nach einer dem Insolvenz-

\footnotetext{
633 Vergleichbar mit der Rücknahme des Einspruchs gem. § 346 ZPO i.V.m. § 516 ZPO. 634 BGH WM 1957, 1225 (1226).

635 AG Bremen NZI 2005, 399 (399); HHK/Preß/Henningsmeier, § 178 Rn.12; Braun/Specovius, § 178 Rn.11; BK/Blersch, § 178 Rn.13; Graf-Schlicker/GrafSchlicker, § 178 Rn.21; HK/Irschlinger, § 178 Rn.4; Kübler/Prütting/Bork/Pape, § 176 Rn.12; KS/Eckardt, S.757 Rn.26; wohl auch MüKo-InsO/Nowak, § 176 Rn.30.

636 Die Rücknahme gegenüber dem Insolvenzgericht oder dem Anmeldenden bejahen: Uhlenbruck/Uhlenbruck, § 178 Rn.8; Gottwald/Eickmann, § 64 Rn.9; MüKoInsO/Schumacher, § 178 Rn.43.

637 Hinsichtlich einer Divergenz zwischen Feststellungs- (§ 178 I InsO) und Tabelleninhalts (§ 178 II InsO).

638 Die Feststellungswirkung des $\S 178$ I, III InsO greift hier, im Gegensatz zum letztmöglichen Zeitpunkt des Widerspruch, gerade nicht. Dazu auch unter E.I.4.b) (S.142ff.).
} 
verfahren entzogenen und schließlich im Feststellungsprozess erfolgten Entscheidung über den erhobenen Widerspruch hinsichtlich dessen Begründetheit oder Unbegründetheit - mit der Folge des Verlustes der formellen Insolvenzgläubigerstellung oder der Feststellung der Insolvenzforderung - rechtskräftig und damit endgültig entschieden wurde und somit nur noch das Feststellungsurteil für die Berichtigung der Tabelle durch den Obsiegenden maßgeblich ist (§ 183 I, II i.V.m. § 178 I 1 InsO).

\section{bb) Beseitigung des Widerspruchs und Betreibungslasten}

Die Beseitigung des Widerspruchs erfolgt grundsätzlich im ordentlichen Klageweg durch Feststellung der Insolvenzforderung zur Tabelle im Feststellungsrechtsstreit gem. §§ 179ff., 184 InsO. ${ }^{639}$ Die Betreibenslast hängt dabei davon ab, ob eine angemeldete Insolvenzforderung bereits durch einen vollstreckbaren Schuldtitel oder durch ein Endurteil tituliert war oder nicht. Diesbezüglich sollen im Folgenden die einzelnen Verfahrenssituationen bei erhobenen Widersprüchen (1) zwischen den Insolvenzgläubigern untereinander und (2) hinsichtlich eines Widerspruchs durch den Schuldner und deren Folge hinsichtlich der Betreibungslast zur Widerspruchsbeseitigung aufgezeigt und erörtert werden. Dabei sollen insbesondere Probleme bei der Wahrung der Schuldnerinteressen hinsichtlich seiner Restschuldbefreiung im Zusammenhang mit angemeldeten (titulierten) Insolvenzforderungen aus vorsätzlich begangener unerlaubter Handlung analysiert und einer (künftigen) Lösungsmöglichkeit zugeführt werden.

\section{(1) Widerspruch durch den Insolvenzgläubiger/-verwalter}

639 Gem. § 183 II InsO obliegt es der obsiegenden Partei, beim Insolvenzgericht die Berichtigung der Tabelle für die spätere Möglichkeit der Erteilung eines vollstreckbaren Tabellenauszugs zu beantragen (§ 201 II 1 InsO). Das Gleiche gilt entsprechend für Feststellungsprozesse gem. § 184 InsO bei einem Schuldnerwiderspruch. 
Ist die Insolvenzforderung nicht tituliert und von dem Insolvenzverwalter oder einem Insolvenzgläubiger bestritten worden, so obliegt es gem. § 179 I InsO dem anmeldenden Insolvenzgläubiger, die Feststellung der Insolvenzforderung zu betreiben. Der Grund hierfür ist ein doppelter: Einerseits braucht es sich der Anmeldende nicht gefallen zu lassen, gegen seinen Willen in einen Feststellungsstreit verwickelt $\mathrm{zu}$ werden; andererseits haben der Insolvenzverwalter und die widersprechenden Insolvenzgläubiger kein Interesse daran, ihrerseits die Feststellung des Nichtbestehens der Insolvenzforderung zu betreiben. Denn nach § 189 I 1 InsO wird die bestrittene Forderung bei der Vereilung ohnehin nicht berücksichtigt, falls nicht der Insolvenzgläubiger binnen einer Ausschlussfrist von zwei Wochen nach der öffentlichen Bekanntmachung der Summe der Forderungen und des für die Verteilung verfügbaren Betrags durch das Insolvenzgericht ( $§ 188$ InsO) nachgewiesen hat, dass und für welchen Betrag Feststellungsklage erhoben oder das Verfahren in dem früher anhängigen Rechtsstreit aufgenommen worden ist. ${ }^{640}$

War die angemeldete Forderung hingegen tituliert, so obliegt die Betreibungslast gem. § 179 II InsO dem Widersprechenden, da dem anmeldenden Gläubiger die Rechtsposition, die er vor Eröffnung des Insolvenzverfahrens im Verhältnis zum Schuldner erlangt hat, auch im Verhältnis zum Widersprechenden zu erhalten ist. ${ }^{641}$ Wird der Widerspruch gegen die titulierte Insolvenzforderung nicht vom Widersprechenden weiterverfolgt, nimmt sie im Gegensatz zur untitulierten zwar dennoch an einer Verteilung teil (§ 189 I InsO), um aber auch die Feststellung seiner Forderung etwa hinsichtlich eines Stimmrechts zu erwirken, wird man dem Insolvenzgläubiger ein insolvenzrechtlich berechtigtes Feststellungsinteresse einräumen müssen, den Widerspruch auch selbst durch Klageerhebung oder Aufnahme eines anhängigen Prozesses (§ 180 InsO) feststellen zu lassen. ${ }^{642}$ Als titulierte Forderung im Sinne des § 179 II InsO sind sämtliche vollstreckbare Schuldtitel oder Endurteile ${ }^{643}$ anzusehen, die

640 Siehe auch unter E.II.3.b) (S.194ff.).

641 MüKo-InsO/Schumacher, § 179 Rn.29.

${ }^{642}$ RGZ 34, 409 (410); 86, 235 (237); BGH NJW 1965, 1523 (1523); Nerlich/Römermann/Becker, § 179 Rn.17; Uhlenbruck/Uhlenbruck, § 179 Rn.16; MüKo-InsO/Schumacher, § 179 Rn.43 m.w.N.

643 Für Einzelheiten siehe MüKo-InsO/Schumacher § 179 Rn.23f. 
im Zeitpunkt der Verfahrenseröffnung bereits vorlagen ${ }^{644}$ und aus denen sich ein entsprechender Zahlungsanspruch gegen den Schuldner ergibt, der identisch mit der zur Tabelle angemeldeten Insolvenzforderung ist.

Fraglich in diesem Zusammenhang ist jedoch, wann eine angemeldete Insolvenzforderung auch hinsichtlich des Forderungsattributs gem. § 174 II InsO als tituliert angesehen werden kann Dies ist jedenfalls unproblematisch dann anzunehmen, wenn sich der qualifizierte Rechtsgrund der vorsätzlich begangenen unerlaubten Handlung aus dem Tenor des Titels ergibt, ${ }^{645}$ der im Wege eines Erkenntnisverfahrens ergangen ist und dem dabei mindestens eine Schlüssigkeitsprüfung des Richters vorausgegangen ist. ${ }^{646}$

Hinsichtlich eines Vollstreckungsbescheids der im Mahnverfahren ergangen ist wird jedoch mit Blick auf die Regelung in § 850f II ZPO zu differenzieren sein. Ein Vollstreckungsbescheid genügt zwar, um die Voraussetzungen eines Zahlungstitels zu erfüllen, er ist jedoch nicht geeignet, die rechtliche Einordnung des in ihm geltend gemachten Anspruchs festzulegen, da der Mahnbescheid allein auf den einseitigen, vom Gericht nicht materiellrechtlich geprüften Angaben des Gläubigers beruht. ${ }^{647}$ So entspricht es zwar dem Sinn und Zweck des Mahnverfahrens, dem Gläubiger hinsichtlich eines bestimmten Zahlungsanspruches (§ 688 I ZPO) einen einfachen und kostengünstigen Weg $\mathrm{zu}$ einem

Vollstreckungstitel zu eröffnen. ${ }^{648}$ Will der Gläubiger es dabei nicht

644 Eine Forderung wird nicht dadurch i.S.d. § 179 II InsO tituliert, dass nach der Eröffnung des Insolvenzverfahrens unter Verletzung der §§ 240, 249 ZPO ein Versäumnisurteil ergeht oder ein Vollstreckungsbefehl erlassen wird. Gem. § 240 ZPO unterbrochene Prozesse sind vielmehr wiederaufzunehmen (§ 180 II InsO). Beachte aber § 249 III ZPO. Dazu ausführlich OLG Köln NJW 1988, 1859 (1859); weiter Braun/Specovius, § 181 Rn.16; MüKo-InsO/Schumacher § 179 Rn.25 m.w.N.

645 Siehe dazu Peters, KTS 2006, 127 (129). Dem Antrag des Gläubigers, die Qualifizierung der Forderung in den Tenor mit aufzunehmen, wird das Gericht hinsichtlich seines Rechtsschutzbedürfnisses bezüglich § 302 Nr.1 InsO regelmäßig folgen.

646 BGH ZInsO 2006, 704 (704f.); für den parallel liegenden Fall des § 850f II ZPO: BGH ZInsO 2005, 538 (538f.); BGHZ 152, 166 (169ff.).

647 Das Prozessgericht ist deshalb nicht an den Vollstreckungsbescheid gebunden. BGH ZInsO 2005, 538 (538f.); BGHZ 152, 166 (169ff.); Baur/Stürner/Bruns, Zwangsvollstreckung, Rn.24.39.

648 Für den Schuldner stellt sich im Mahnverfahren nicht die Frage, ob er Widerspruch oder Einspruch nur deshalb einlegen soll, um eine Abänderung der rechtlichen Einordnung zu erreichen, vor allem dann nicht, wenn der Schuldner nach eigener 
belassen, sondern weitergehend das Vollstreckungsprivileg des § 850f II ZPO in Anspruch nehmen, so muss hierfür ein Feststellungsurteil hinsichtlich der Berechtigung eines erweiterten Vollstreckungszugriffs erwirkt werden, das im ordentlichen Verfahren ergeht und mindestens eine gerichtliche Schlüssigkeitsprüfung voraussetzt. ${ }^{649}$ Gilt diese Voraussetzung der Schlüssigkeitsprüfung für $\S$ 850f II ZPO, so hat dies erst recht entsprechend für die Qualifikation einer Insolvenzforderung aus einer vorsätzlich begangenen unerlaubten Handlung im Sinne der §§ 174 II, 302 Nr.1 InsO zu gelten. ${ }^{650}$ Denn die Konsequenzen des $\S 302$ Nr.1 InsO übersteigen in ihren Auswirkungen noch diejenigen des § 850f II InsO, und zwar dahingehend, dass im Gegensatz zur (lediglichen) Beschränkung der Pfändungsgrenze des Arbeitseinkommens des Schuldners (§ 850c ZPO) die Feststellung des Forderungsattributs im Insolvenzverfahren unter Umständen für den Schuldner zur Folge hat, dass ihm ab einer gewissen Forderungshöhe der Weg zur (vollständigen) Restschuldbefreiung und damit zu einem wirtschaftlichen Neuanfang dauerhaft verbaut ist. ${ }^{651}$ Und während Ersteres immerhin von einer weiteren Ermessensentscheidung des Vollstreckungsgerichts abhängt, wäre der Verlust des Neuanfanges unausweichlich. Daher bleibt festzuhalten, dass die Vorlage eines Vollstreckungsbescheids mit einer entsprechenden Vermerkung hinsichtlich des qualifizierten Rechtsgrundes, der nicht im Wege einer richterlichen Schlüssigkeitsprüfung ergangen ist, nicht als Titel über den qualifizierten Rechtsgrund angesehen werden kann. Entsprechend liegt in diesem Fall auch die Betreibungslast hinsichtlich des Forderungsattributs beim widerspruchsbetroffenen Gläubiger und nicht beim bestreitenden Schuldner (§ 184 I InsO) oder Insolvenzgläubiger (§ 179 I InsO). ${ }^{62}$

Auffassung jedenfalls im Ergebnis den geforderten Betrag schuldet. Die rechtlichen Folgen, welche die Titulierung einer derartigen Forderung nach sich zieht, wird ein Schuldner regelmäßig nicht überblicken. Vgl. BGH ZInsO 2006, 704 (704f.). Dadurch wäre es für die Gläubiger bei dem geringsten Anzeichen einer vorsätzlich begangenen unerlaubten Handlung ein Leichtes, den privilegierten Rechtsgrund durch einen (bloßen) Vollstreckungsbescheid „betiteln“ zu lassen.

BGH ZInsO 2005, 538 (538f.); BGHZ 152, 166 (169ff.); MüKo-InsO/Nowak, $\S 175$ Rn.19; Ahrens, EWiR 2006, 541 (542).

650 Hinsichtlich dieser Übertragungsmöglichkeit siehe nur BGH ZInsO 2006, 704 (704f.) m.w.N.

651 Anm. Grote zu BGH ZInsO 2005, 538 (540); Peters, KTS 2006, 127 (127).

652 Es obliegt grundsätzlich dem Insolvenzgläubiger, den Nachweis für das von ihm behauptete Privileg zu erbringen. BGH ZInsO 2006, 704 (704f.). 
Grundsätzlich beseitigt die gerichtliche Feststellung der bestrittenen Insolvenzforderung gegenüber dem Bestreitenden ( $\S 183$ I InsO) den Widerspruch im Sinne des § 178 I 1 InsO. Umgekehrt verliert der widersprechende Insolvenzgläubiger mit Rechtskraft der Feststellung des Nichtbestehens seiner eigenen Forderung im Feststellungsrechtsstreit gem. §§ 179ff. InsO seine formelle Insolvenzgläubigerstellung und damit auch sämtliche Teilnahmerechte im Insolvenzverfahren, ${ }^{653}$ so dass seine bis dahin erhobenen Widersprüche unwirksam werden und infolgedessen einem gegnerischen Feststellungsantrag hinsichtlich seines Widerspruchs in einem bereits erhobenen Feststellungsprozess stattzugeben ist. ${ }^{654}$ Ist dagegen zunächst über den Widerspruch des Insolvenzgläubigers einer bestrittenen Forderung gegen das Teilnahmerecht eines anderen abschließend entschieden worden, so hat es keinen Einfluss auf das Ergebnis dieses Verfahrens, wenn später festgestellt wird, dass auch dem Widersprechenden kein Teilnahmerecht zusteht. Insofern bleibt es für den Gläubiger, dessen Forderung nicht zur Tabelle durch Feststellungsurteil festgestellt worden ist, auch dann bei dem Verlust seiner formellen Insolvenzgläubigerstellung, wenn auch der Anspruch seines gegnerisch Bestreitenden nicht als Insolvenzforderung festgestellt wird, ${ }^{655}$ weil beide Gläubiger nicht berechtigt sind, mit ihren jeweils angemeldeten Forderungen am Insolvenzverfahren teilzunehmen. Eine Rückwirkung ist somit ausgeschlossen.

Nimmt der Widersprechende hingegen vor Erhebung einer Feststellungsklage seine angemeldete Forderung vom Insolvenzverfahren zurück oder wurde seine fehlende Teilnahmeberechtigung bereits in einem anderen Feststellungsverfahren hinsichtlich eines anderen Widerspruchs rechtskräftig festgestellt, so wird der Insolvenzgläubiger der widerspruchsbetroffenen Forderung mit Hinweis auf das in diesen Fällen eindeutig nachweisbar fehlende Widerspruchsrecht des Widersprechenden - falls er hiervon im Vorfeld der eigenen Feststellungsklage jedoch überhaupt Kenntnis erlangt - die Tabellenberichtigung beim

653 Siehe bereits unter E.I.4.e) (S.163ff.).

654 Wie die Rücknahme des Widerspruchs während des Verfahrens führt auch der Verlust des Widerspruchsrechts währenddessen zur Erledigung des Feststellungsverfahrens in der Hauptsache. Vgl. BGH WM 1957, 1225 (1226) zur Rücknahme; weiter Jaeger/Weber, KO, § 141 Rn.8; Uhlenbruck/Uhlenbruck, § 178 Rn.8 m.w.N.

655 Kübler/Prütting/Bork/Pape, § 176 Rn.10; Jaeger/Weber, KO, § 141 Rn.8. 
Insolvenzgericht beantragen können. ${ }^{656}$ Auch das Erlöschen der eigenen Insolvenzforderung des Bestreitenden führt grundsätzlich zum Verlust seiner formellen Insolvenzgläubigerstellung und damit zur Unwirksamkeit seiner erhobenen Widersprüche. ${ }^{67}$ Sollte in diesem Fall jedoch der Gläubiger der erloschenen Forderung die Berichtigung der Insolvenztabelle hinsichtlich seines nunmehr unwirksamen Widerspruchs durch das Insolvenzgericht nicht auf Eigenantrag oder mit seiner erklärten Zustimmung auf Antrag des Widerspruchsbetroffenen vornehmen lassen, wird eine Beseitigung des Widerspruchs nur im Feststellungsstreit erreicht werden können. Hierfür spricht, dass über die materiellrechtliche Frage hinsichtlich des Bestehens oder Erlöschens der Insolvenzforderung des Widersprechenden im Streitfall gerade das Prozessgericht zu entscheiden hat, nicht aber das Insolvenzgericht im Rahmen eines Verfahrens zur Berichtigung der Tabelle. Daher kann in diesem Fall eine Beseitigung des „Widerspruchs“ nur durch seine Rücknahme oder im Feststellungsstreit erreicht werden. ${ }^{658}$

\section{(2) Widerspruch durch den Schuldner}

Im Fall des Schuldnerwiderspruchs nimmt die Insolvenzforderung wegen ihrer grundsätzlichen Feststellung (vgl. § 178 I 2 InsO) - bei einem gleichzeitig unterlassenen Widerspruch seitens des Insolvenzgläubigers/verwalters - gleichwohl am Verteilungsverfahren gem. §§ 187ff. InsO teil. Der Schuldnerwiderspruch verhindert jedoch, dass nach Beendigung des Insolvenzverfahrens die Eintragung der Feststellung in die Tabelle gem. § 201 II 1 InsO Vollstreckbarkeit und Rechtskraft gegenüber dem Schuldner persönlich erzeugt. Die Betreibenslast zur Beseitigung eines Schuldnerwiderspruchs ist dabei umfassend in § 184 InsO geregelt.

Der Insolvenzgläubiger einer nicht titulierten Forderung hat gem. § 184 I InsO Klage auf Feststellung seiner Insolvenzforderung gegen den Schuldner persönlich zu erheben oder einen gem. $\S 240$ ZPO

656 Braun/Specovius, § 178 Rn.12; Gottwald/Eickmann, § 64 Rn.13.

657 Gottwald/Eickmann, $\S 64 \quad$ Rn.12; Uhlenbruck/Uhlenbruck, $\S 178$ Rn.9; Braun/Specovius, § 178 Rn.11; Jager/Weber, KO, § 141 Rn.8.

658 Gottwald/Eickmann, § 64 Rn.14; MüKo-InsO/Schumacher, § 178 Rn.46 m.w.N.; siehe auch KO-Motive, S.362 = Hahn, KO, S.326. 
unterbrochenen Rechtsstreit gegen den Schuldner wieder aufzunehmen mit dem Ziel, die nachinsolvenzliche Vollstreckung aus der Tabelle zu ermöglichen. ${ }^{659}$ Dies gilt entsprechend auch hinsichtlich eines isolierten Widerspruchs des Schuldners gegen das Forderungsattribut der vorsätzlich begangenen unerlaubten Handlung. ${ }^{600}$ Der Gesetzgeber hat im Rahmen des $\S 184$ I InsO lediglich eine positive Feststellungsklage des Insolvenzgläubigers vorgesehen, ${ }^{661}$ so dass es an einem Rechtsschutzbedürfnis hinsichtlich einer negativen Feststellungsklage des Schuldners regelmäßig fehlt, ${ }^{662}$ insbesondere weil der Schuldner durch die Erhebung seines Widerspruch gem. § 201 II 1 InsO hinreichend vor einer nachinsolvenzlichen Vollstreckung geschützt ist und es einer ausdrücklichen gerichtlichen Feststellung insofern nicht bedarf. Im Falle eines dennoch erfolgenden nachinsolvenzlichen Vollstreckungsversuchs kann sich der Schuldner mit der Vollstreckungsgegenklage gem. § 767 ZPO wehren. Hat der Schuldner versäumt, die Forderung im Prüfungstermin oder im schriftlichen Verfahren zu bestreiten, kann er dieses Versäumnis - genauso wie alle übrigen Widerspruchsberechtigten - ebenfalls nicht dadurch nachholen, dass er nachträglich eine negative Feststellungsklage erhebt. ${ }^{63}$ Dies würde gänzlich dem Sinn und Zweck des systematisierten Ablaufs des insolvenzrechtlichen Prüfungsverfahrens widersprechen, durch die Prüfungsergebnisse eine verbindliche Grundlage für das gesamte Insolvenzverfahren $\mathrm{zu}$ schaffen. Im Gegensatz $\mathrm{zu}$ den übrigen

659 Die $\S \S 179$ - 183 I und III InsO sind dabei nicht anwendbar, weil es bei der Feststellungsklage gem. § 184 I InsO gerade nicht (mehr) um die Teilhabe des Insolvenzgläubigers mit seiner Forderung an der Masse in Bezug zu den übrigen Insolvenzgläubigern, sondern nunmehr um dessen Anspruch gegen den Schuldner persönlich geht. Somit sind die allgemeinen prozessualen Regeln anzuwenden. Anders wiederum bei der Feststellungsklage gegen den Schuldner als Eigenverwalter. - MüKo-InsO/Schumacher, § 184 Rn.3; Nerlich/Römermann, § 184 Rn.11; Kübler/Prütting/Bork/Pape, § 184 Rn.12 m.w.N.

660 BGH ZInsO 2007, 265 (265); BGH NZI 2006, 536 (536ff.); OLG Rostock ZInsO 2006, 1175 (1175f.); LG Potsdam ZinsO 2006, 615 (615f.); MüKo-InsO/Schumacher, § 184 Rn.8; HHK/Herchen, § 184 Rn.13; Hattwig, ZinsO 2004, 636 (637f.); Braun/Specovius, § 184 Rn.6; Kübler/Prütting/Bork/Pape, § 184 Rn.98 m.w.N.

661 Insofern ist mit Hinweis auf $\S 87$ InsO auch der teilweise angeführten Möglichkeit einer Leistungsklage gegen den Schuldner (vgl. etwa MüKo-InsO/Schumacher, § 184 Rn.6f.) zu widersprechen. Für die ausschließliche Zulässigkeit der Feststellungsklage im Insolvenzverfahren siehe auch Kübler/Prütting/Bork/Pape, § 184 Rn.16f. m.w.N. Siehe auch bereits unter B. (S.4ff.).

662 Vgl. BGH ZInsO 2004, 88 (89); weiter OLG Hamm, ZInsO 2004, 683 (683); MüKo-

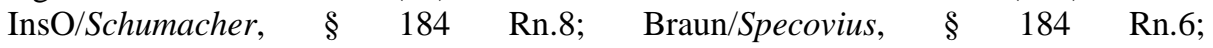
Kübler/Prütting/Bork/Pape, § 184 Rn.18 m.w.N.

663 MüKo-InsO/Schumacher, § 184 Rn.8; Kübler/Prütting/Bork/Pape, § 184 Rn.18f. m.w.N. 
Widerspruchsberechtigten besteht für den Schuldner bei der Versäumnis des Prüfungstermins ${ }^{664}$ jedoch die Möglichkeit der Wiedereinsetzung in den vorigen Stand gem. § 186 InsO. ${ }^{665}$ Ansonsten stehen dem Schuldner bei einem unterlassenen Widerspruch nach Beendigung des Insolvenzverfahrens ebenso wie allen übrigen Verfahrensteilnehmern nur die allgemeinen zivilprozessualen Rechtbehelfe gegen die gem. § 178 I, III InsO rechtskräftige Feststellung der Insolvenzforderung zur Verfügung. ${ }^{666}$ Ist die vom Schuldner bestrittene Insolvenzforderung hingegen tituliert, so obliegt es neuerdings gem. § 184 II 1 InsO dem Schuldner, binnen einer Frist von einem Monat, die mit dem Prüfungstermin oder im schriftlichen Prüfungsverfahren mit dem Bestreiten der Forderung beginnt, den Widerspruch $\mathrm{zu}$ verfolgen. Ansonsten gilt sein Widerspruch nach fruchtlosem Ablauf dieser Frist als nicht erhoben (§ 184 II 2 InsO), worauf das Insolvenzgericht den Schuldner gem. § 184 II 3 InsO hinzuweisen hat. Mit dieser Regelung werden sich die bisher streitig gewesenen Fragen erledigen, ob und wann der Insolvenzgläubiger im Falle eines titulierten Anspruchs für eine eigene Klage überhaupt das notwendige Feststellungsinteresse besitzt. ${ }^{667}$

Das Insolvenzgericht muss wegen der Folgen einer Fristversäumnis jedoch als verpflichtet angesehen werden, seiner Hinweispflicht gem. § 184 II 3 InsO bei einem erhobenen Widerspruch des Schuldners bereits unmittelbar im Prüfungstermin bzw. im schriftlichen Prüfungsverfahren nach Erhebung des Widerspruchs durch Zustellung des Hinweises nachzukommen ${ }^{668}$ und nicht erst bei Erteilung eines Tabellenauszugs, wie es der Wortlaut des $\S 184$ II 3 InsO bestimmt. Die Folge einer Fristversäumnis des Schuldners ist mit der dadurch bedingten Feststellung der Insolvenzforderung und ihrer Vollstreckbarkeit nach dem Insolvenzverfahren gegen ihn persönlich

664 Im schriftlichen Prüfungsverfahren wird durch entsprechende Bekanntgabe und Setzung einer Widerspruchsfrist ein „Versäumnis“ i.S.d. § 186 InsO regelmäßig nicht angenommen werden können. Siehe auch unter E.II.5. (S.200ff.).

665 Siehe zur Widerspruchserhebung im Prüfungstermin bereits ab E.I.4.b) (S.142ff.).

666 Siehe dazu unter E.III.7. (S.234f.).

667 Es erscheint zudem unbillig, dass der Gläubiger trotz eines erstrittenen Titels nochmals prozessieren muss und auch bei einer erfolgreichen Prozessführung Gefahr läuft, wegen der wirtschaftlichen Situation des Schuldners seine Kostenerstattungsansprüche nicht oder nur schwer durchsetzen zu können. - Vgl. Begr. zu Nr.23 (§ 184 InsO) RegE, BT-Drucks. 16/3227, S.21.

668 Durch die Möglichkeit der Protokollierung des Hinweises des Insolvenzgerichts im Prüfungstermin bzw. dessen Zustellung beim schriftlichen Verfahren wird zudem der Nachweisbarkeit des Hinweises Rechnung getragen. 
(§§ 184 II 2, 201 II 1 InsO) in diesem Fall besonders schwerwiegend, weil es sich hierbei in ausdrücklicher Anlehnung an den § 878 I ZPO nicht um eine Notfrist handelt, ${ }^{669}$ die dem Schuldner die Wiedereinsetzung in den vorigen Stand gem. § 186 InsO ermöglichen könnte. ${ }^{670}$ Für die (nötige) Rechtssicherheit des Schuldners wäre es daher besser gewesen, den Fristbeginn an den Zugang des Hinweises und nicht direkt an die Beendigung des Prüfungstermins bzw. an den Widerspruch im schriftlichen Verfahren zu knüpfen. ${ }^{671}$

Nicht ganz unproblematisch im Hinblick auf die Neuregelung in § 184 II InsO ist jedoch der Umgang mit dem isolierten Widerspruch des Schuldners gegen das Forderungsattribut der vorsätzlich begangenen unerlaubten Handlung. In diesen Fällen ist hinsichtlich der Betreibungslast nämlich danach zu unterscheiden, ob das Forderungsattribut von dem Urteilstenor oder den tragenden mit in Rechtskraft erwachsenen Entscheidungsgründen mit umfasst ist, oder nicht. Bei einer fehlenden Mittitulierung oder einem lediglich vorhandenen Vollstreckungsbescheid ist die Betreibungslast - aufgrund der vorangegangenen Ausführungen zur generellen Titulierung und der Bindungswirkung eines Vollstreckungsbescheids - nicht dem Schuldner aufzuerlegen, da in diesen Fällen hinsichtlich des Forderungsattributs eine rechtskräftige Titulierung bzw. Bindungswirkung aufgrund fehlender richterlichen Schlüssigkeitsprüfung gerade (noch) nicht vorliegt. In diesem Fall ist die Situation genauso zu beurteilen, als läge gar kein Titel für das Forderungsattribut vor. ${ }^{672}$ Ist das Forderungsattribut aber noch gar nicht tituliert, so gibt es auch keinen Anlass, § 184 II InsO anzuwenden, da dessen Zweck überhaupt nicht berührt ist, wenn noch gar keine gerichtliche Entscheidung darüber vorliegt, ob eine vorsätzlich begangene unerlaubte Handlung des Schuldners gegeben ist. Daher ist es Sache des

669 So ausdrücklich Begr. zu Nr.23 (§ 184 InsO) RegE, BT-Drucks. 16/3227, S.21.

670 Hinsichtlich der Monatsfirst des § 184 II InsO ist daher auf die Rechtslage des $\S 878$ I ZPO zurückzugreifen. Vgl. auch HHK/Herchen, § 184 Rn.11b. Für eine Widereinsetzung gem. § 186 InsO hingegen Kübler/Prütting/Bork/Pape § 184 Rn.30; MüKo-InsO/Schumacher, § 184 Rn.8c.

671 Vgl. auch Kübler/Prütting/Bork/Pape § 184 Rn.30.

672 Würde man es hingegen für entscheidend halten, dass die Insolvenzforderung überhaupt tituliert ist [so wohl Hattwig/Richter, ZVI 2006, 373 (375ff.)], widerspräche dies dem Sinn und Zweck des § 184 II InsO, mit dem auch verhindert werden soll, dass der Schuldner grundlos eine Insolvenzforderung bestreitet, obwohl für diese bereits ein Titel vorliegt. - Kübler/Prütting/Bork/Pape § 184 Rn.109. 
Insolvenzgläubigers, gem. § 184 I InsO die Feststellung zu betreiben, dass der Anspruch aus einer vorsätzlich begangenen unerlaubten Handlung des Schuldners stammt. ${ }^{673}$ Sollte sich das Forderungsattribut hingegen aus dem Tenor oder den tragenden mit in Rechtskraft erwachsenen Gründen der Entscheidung aufgrund einer gerichtlichen Sachprüfung ergeben, so obliegt die Betreibungslast gem. § 184 II InsO dem Schuldner. ${ }^{674}$

Bislang wenig behandelt in diesem Zusammenhang ist jedoch die Frage, bis zu welchem Zeitpunkt eine Feststellungsklage gemäß dem bereits angesprochenen § 184 I InsO zur Beseitigung des Schuldnerwiderspruchs vom Insolvenzgläubiger erhoben bzw. ein unterbrochener Prozess aufgenommen werden kann, da eine Frist - im Gegensatz zum Fall des $\S 184$ II InsO - hier vom Gesetzgeber gerade nicht vorgesehen ist. Diese Frage kann - wie sich nachfolgend zeigen wird - für den Schuldner von entscheidender Bedeutung sein, wenn er einen Antrag auf Restschuldbefreiung gestellt hat.

Eine analoge Anwendung der Monatsfrist des $\S 184$ II InsO auf $\S 184$ I InsO wird mangels ausfüllungsbedürftiger Regelungslücke grundsätzlich ausgeschlossen sein, da die Monatsfrist vom Gesetzgeber in bewusster Kenntnis des Meinungsstreits um die mögliche Befristung der Feststellungsklage allein für titulierte Forderungen in $\S 184$ II InsO eingeführt wurde. ${ }^{675}$

Als Klagefrist im Rahmen des § 184 I InsO wird daher vorgeschlagen, die Frist des $\S 189$ I InsO entsprechend heranzuziehen. ${ }^{676}$ Gegen die entsprechende Maßgeblichkeit der Frist des § 189 I InsO spricht jedoch, dass weder der vollstreckungshindernde Widerspruch des Schuldners mit dem feststellungshindernden Widerspruch der übrigen Verfahrensteilnehmer noch Sinn und Zweck der Frist des § 189 I hinsichtlich der Berücksichtigung der bis dahin nicht einmal festgestellten Insolvenzforderung ins Schlussverzeichnis mit der Situation und dem

So auch HHK/Herchen, § 184 Rn.16b; Kübler/Prütting/Bork/Pape § 184 Rn.108.

674 In diesem Fall wird das Prozessgericht, das über die Feststellungsklage zu entscheiden hat, an die entsprechende Feststellung aus dem Titel gebunden sein, so dass dem Schuldner nur noch die entsprechenden Rechtsmittel gegen den Titel zur Verfügung stehen.

675 Siehe zum Kenntnisstand des Gesetzgebers die Begr. zu Nr.23 (§ 184 InsO) RegE, BT-Drucks. 16/3227, S.21.

${ }^{676}$ Kübler/Prütting/Bork/Pape, § 184 Rn.110f.; vgl. auch Breutigam/Kahlert ZInsO 2002, 469 (469f.). 
Feststellungsinteresse des Insolvenzgläubigers bei einem Schuldnerwiderspruch vergleichbar und damit auch nicht auf die Regelungslücke des $\S 184$ I InsO übertragbar sind. Im Fall des § 184 I InsO geht es zwar auch um die Feststellung der Forderung zur Insolvenztabelle, aber nicht hinsichtlich der Forderung als Insolvenzforderung im Sinne des § 178 I, III InsO und ihrer Abwicklung und Befriedigung im Insolvenzverfahren, worauf sich $\S 189$ I InsO bezieht, ${ }^{677}$ sondern um die Feststellung im Sinne des § 201 II 2 i.V.m. § 178 III InsO als vollstreckbarer Anspruch des Insolvenzgläubigers gegen den Schuldner persönlich nach Beendigung des Insolvenzverfahrens. ${ }^{678}$ Die Anwendung der Frist des $\S 189$ I InsO hätte darüber hinaus insolvenzverfahrenstechnisch zur Folge, dass für denjenigen Insolvenzgläubiger, der seine Forderung erst nachträglich im Schlusstermin anmeldet bzw. dessen Insolvenzforderung erst im Schlusstermin zur Prüfung gestellt werden $k^{2} n^{679}$ und sodann vom Schuldner bestritten wird, aufgrund der (längst) versäumten Frist des § 189 I InsO keine Möglichkeit mehr bestehen würde, gegen den Schuldnerwiderspruch vorzugehen. Aus dem gleichen Grund wird man daher auch die Feststellungsklage gem. § 179 I InsO gegen einen feststellungshindernden Widerspruch - für die ebenfalls eine Fristenregelung zur Klageerhebung fehlt - nicht generell an die Frist des § 189 I InsO binden können, ${ }^{680}$ da auch in diesem späten Verfahrensstadium grundsätzlich noch ein Feststellungsinteresse des Insolvenzgläubigers hinsichtlich einer Titulierung seiner Forderung und auch - damit einhergehend - Zuweisung eines Stimmrechts besteht. ${ }^{681}$ Ermöglicht richtigerweise noch der Schlusstermin die letzte Möglichkeit zur Anmeldung einer Insolvenzforderung, so sind dem ordnungsgemäß anmeldenden und damit formellen Insolvenzgläubiger auch alle damit verbundenen Verfahrensrechte zuzusprechen, wozu auch das Recht auf die Möglichkeit der Beseitigung eines Widerspruchs - gleich ob vom

Siehe zur Berücksichtigung der Insolvenzforderung im Schlussverzeichnis unter E.II.3.b) (S.194ff.).

678 So scheinbar auch Kübler/Prütting/Bork/Pape, § 184 Rn.4. Siehe in diesem Zusammenhang auch die Ausführungen zu den unterschiedlichen Widerspruchsrichtungen von Insolvenzgläubiger und Schuldner unter E.I.4.c)bb) (S.145ff.).

Siehe dazu unter E.II.3.b) (S.194ff.).

680 So aber grundsätzlich MüKo-InsO/Schumacher, § 179 Rn.9f. m.w.N. zum Meinungsstand.

681 Dazu auch unter E.II.3.a) (S.192ff.). 
Schuldner oder vom Insolvenzgläubiger/Insolvenzverwalter erhoben - im dafür vorgesehenen Feststellungsverfahren gem. §§ 179ff. InsO gehören muss. Das Feststellungsinteresse des Anmeldenden begründet nämlich nicht die lediglich zu gewährende Anmeldungsmöglichkeit im Schlusstermin, sondern gerade die Feststellungsmöglichkeit und die damit verbundene Sicherung seiner zuzusprechenden Verfahrensrechte durch die Titulierung seiner Insolvenzforderung. Als Konsequenz aus Feststellungsinteresse und Feststellungsmöglichkeit ist - wie in jedem früheren Stadium des Verfahrens zweifelsfrei der Fall - auch in diesem verspäteten Fall jedem formellen Insolvenzgläubiger der weiterführende prozessuale Feststellungsweg gem. §§ 179ff. InsO zu eröffnen. Denn Anmeldungs/Feststellungsinteresse - Prüfungstermin - Feststellungsklage sind insolvenzverfahrensrechtlich unmittelbar miteinander verbunden; und dem widerspruchsbetroffenen Insolvenzgläubiger kann sein Interesse daher nicht abgesprochen werden. Wäre dies anders, wäre ein umfassend sachund interessensgerechtes Feststellungsverfahren, wie es die Gesamtsystematik des Insolvenzverfahrens vorgibt, für den so verspätet Anmeldenden überhaupt nicht möglich und von vornherein zum Scheitern verurteilt. Daher ist die Übertragung der Frist des § 189 I InsO auf die allgemeine Feststellungsklage gem. $§ \quad 184$ I InsO aufgrund des unterschiedlichen Sinns und Zwecks, aber auch aus insolvenzverfahrensrechtlichen Gründen nicht möglich und folglich abzulehnen. ${ }^{682}$

Auch der Anknüpfungspunkt einer möglichen Klageerhebung bis zur Entscheidung über die Restschuldbefreiung gem. §§ 291, 289 I 1 InsO wird im Rahmen des $\S 184$ I InsO abzulehnen sein, ${ }^{683}$ da der Beschluss hierüber regelmäßig im Schlusstermin ergeht, wodurch dem erst im Schlusstermin anmeldenden Insolvenzgläubiger bzw. dessen erst dort zur Prüfung gestellten Insolvenzforderung die Möglichkeit der Verfolgung eines eventuell erhobenen Widerspruchs zu diesem Zeitpunkt ebenfalls bereits genommen wäre. Zudem ist nicht jedes Insolvenzverfahren zwangsweise mit einem Antrag auf Restschuldbefreiung verbunden, so

682 Gleiches wird auch hinsichtlich eines Feststellungsinteresses gem. § 179 I InsO zu gelten haben. Auch wenn die Frist des § 189 I InsO nach Sinn und Zweck auf § 179 I InsO anwendbar ist, wird man deren Anwendung wegen des neben dem Befriedigungsinteresse bestehenden Titulierungsinteresses gleichwohl ablehnen müssen.

683 Hierfür Hattwig ZInsO 2004, 636 (638f.). 
dass dieser Anknüpfungspunkt auch keine einheitliche Anwendung im Insolvenzverfahren finden würde/ könnte.

Folglich scheint ein eindeutiger Anknüpfungspunkt für eine Klagefrist des $\S 184 \mathrm{I}$ InsO, die sich innerhalb des noch nicht aufgehobenen/beendeten Insolvenzverfahrens bewegt, ohne dem formellen Insolvenzgläubiger seine elementaren Verfahrensrechte zu nehmen, nicht handhabbar. Insofern scheint hier eine Regelungslücke vorzuliegen, die ohne gesetzliche Regelung nicht geschlossen werden kann. Fraglich ist daher, wie man diesbezüglich gleichwohl zu einem interessengerechten und ausdifferenzierten Lösungsansatz kommen könnte.

Zumindest in den meisten Fällen, in denen der Schuldner einen Restschuldbefreiungsantrag gestellt hat, wird grundsätzlich unproblematisch davon auszugehen sein, dass ein Feststellungsinteresse des Insolvenzgläubigers, der seine vom Schuldner widersprochene Forderung nicht zugleich auf das Forderungsattribut gestützt hat, mit Erteilung oder Versagung der Restschuldbefreiung am Ende der Wohlverhaltensphase nicht mehr gegeben sein wird. Der Insolvenzgläubiger kann in diesem Fall - wie bereits ausgeführt ${ }^{684}$ - nicht zu einer (vorzeitigen) Feststellungsklage gezwungen werden, die nicht auch absehbar - aufgrund der Ungewissheit über die Erteilung oder Versagung der Restschuldbefreiung - zum gewünschten Erfolg führt. Bei einer Erteilung der Restschuldbefreiung, die allein in der Hand des Schuldners durch sein „Wohlverhalten“ liegt, unterfällt die Insolvenzforderung der Restschuldbefreiung, wodurch das Feststellungsinteresse des Insolvenzgläubigers zweifelsohne entfällt. Zeichnet sich im Vorhinein jedoch ab, dass die Restschuldbefreiung versagt wird oder auch nur versagt werden könnte, so ist es dem an einer Titulierung interessierten Insolvenzgläubiger zuzumuten, sich stets über den Verlauf des Verfahrens auf dem Laufenden zu halten und entsprechend vor der Entscheidung über die Restschuldbefreiung Feststellungsklage gegen den Schuldner zu erheben.

\footnotetext{
684 Siehe in diesem Zusammenhang auch hinsichtlich der Hemmung der angemeldeten Insolvenzforderung bereits unter D.VI.4. (S.115ff.). - So scheinbar auch FK/Ahrens, $\S 302$ Rn.11; vgl. auch OLG Rostock ZVI 2005433 (434) und Vallender NZI 2002, 110 (112).
} 
Anders sieht es hingegen bei den vom Schuldner bestrittenen Insolvenzforderungen aus, die (zusätzlich) mit dem Forderungsattribut der vorsätzlich begangenen unerlaubten Handlung angemeldet worden sind. Zwar ist auch hier zunächst gleichermaßen anzunehmen, dass der widerspruchsbetroffene Insolvenzgläubiger zumindest bis $\mathrm{zu}$ einer Entscheidung über die Restschuldbefreiung die Feststellungsklage gem. $\S 184$ I InsO gegen den Schuldner erhoben oder entsprechend wieder aufgenommen haben muss, da seine Forderung ohne Feststellung des Forderungsattributs zur Tabelle ebenfalls von der Restschuldbefreiung mit ihrer Erteilung erfasst wird (§§ $201 \mathrm{II}$ 1, $302 \mathrm{Nr} .1$ InsO) ${ }^{685}$ Doch gerade diese, im Rahmen des Restschuldbefreiungsverfahrens bis zu sechs Jahre dauernde Ungewissheit des Schuldners über seine $\mathrm{zu}$ erwartende Nachhaftung durch Insolvenzforderungen aus vorsätzlich begangener unerlaubter Handlung - die bei ihrer Feststellung auch gegenüber dem Schuldner gem. $\S 302$ Nr.1 InsO von der Restschuldbefreiung ausgenommen sind - sollte dem Schuldner im Rahmen der Einführung und Anpassung der §§ 174 II, 175 II, 302 Nr.1 InsO durch eine frühzeitige, nach Möglichkeit noch während des laufenden Insolvenzverfahrens durchzuführende Klärung genommen werden. Der Schuldner soll dadurch frühzeitig abschätzen können, ob sich für ihn die Unterwerfung unter die weitgreifenden Regelungen des Restschuldbefreiungsverfahrens überhaupt lohnt. ${ }^{686}$ Insofern erfolgt durch die eingeführten Regelungen zwar eine umfangreiche und frühzeitige „Warnung“ und der Hinweis für den Schuldner auf seine Widerspruchsmöglichkeit und auf die Konsequenzen einer mit dem Forderungsattribut angemeldeten und unwidersprochen gebliebenen Insolvenzforderung ( $§ 175$ II InsO), jedoch wird durch den derzeitigen Regelungsumfang - bei einem daraufhin erhobenen Widerspruch des Schuldners - ohne eine weiterführende eindeutige Regelung, etwa hinsichtlich einer Klagefrist im Rahmen der Feststellungsklage gem. $\S 184$ I InsO, ihr eigentliches bzw. weiterführendes Ziel einer frühzeitigen und letztendlichen Gewissheit des Schuldners über den Status seiner Nachhaftung nicht erreicht. Und diese Klarheit ist nicht nur wünschenswert, sondern sollte wegen der dahinter

685 FK/Ahrens, § 302 Rn.10d; Uhlenbruck/Uhlenbruck, § 302 Rn.18; FK/Kießner $\S 175$ Rn.18; Kübler/Prütting/Bork/Pape, § 174 Rn.46.

686 Dazu bereits unter D.IV.3.a) (S.44ff.). 
stehenden gesetzgeberischen Intention als selbstverständlich angesehen werden. Aus vergleichbaren Gründen schaffte der Gesetzgeber auch Klarheit über die Betreibungslast gekoppelt mit einer Ausschlussfrist im Rahmen des § 184 II InsO im Fall des Schuldnerwiderspruchs bei titulierten Insolvenzforderungen, „um alsbald Klärung über die Wirkung des Widerspruchs zu erhalten“. ${ }^{687}$ Wenn eine solche Ausschlussfrist für die Gewissheit des Insolvenzgläubigers als angebracht erachtet wird, dann sollte man dies erst recht für den Schuldner im Hinblick auf seine Gewissheit über seine eventuelle Nachhaftung und der angedachten schuldnerschützenden Regelungsfunktion der §§ 174 II, 175 II, 302 Nr.1 InsO vermuten, zumindest wenn es die Beseitigung eines Schuldnerwiderspruchs gegen eine Insolvenzforderung mit untituliertem Forderungsattribut ${ }^{688}$ betrifft. Darüber hinaus wäre ein Gleichklang von $\S 184$ II InsO und § 179 II InsO - wenn der Gesetzgeber § 184 II InsO schon an den $\S 179$ II InsO anlehnt ${ }^{699}$ - nur konsequent gewesen, da es keinen sachlichen Grund gibt, den bestreitenden Schuldner schlechter zu stellen als den bestreitenden Insolvenzgläubiger oder Insolvenzverwalter. ${ }^{690}$ Solange es jedoch an einer einheitlichen Regelung hinsichtlich einer Klage- bzw. Ausschlussfrist im Rahmen des § 184 I InsO fehlt, wird unter Berücksichtigung der gesetzgeberischen Intention hinsichtlich der Begrenzung des Feststellungsinteresses eines widerspruchsbetroffenen Insolvenzgläubigers einer auf einer vorsätzlich begangenen unerlaubten Handlung beruhenden Forderung im Rahmen des $\S 184$ I InsO wie nachfolgend zu differenzieren sein, um eine möglichst sachgemäße und praktikable Trennungslinie zu schaffen:

Der Insolvenzgläubiger, dessen Forderung in einem Prüfungstermin oder schriftlichen Prüfungsverfahren vor dem Schlusstermin geprüft und vom Schuldner bestritten wurde, hat bis zum Schlusstermin - in dem regelmäßig gem. §§ 289 I, 292 I InsO über die Restschuldbefreiung zu entscheiden ist - die Möglichkeit Feststellungsklage zu erheben. Dem

\footnotetext{
Vgl. Begr. zu Nr.23 (§ 184 InsO) RegE, BT-Drucks. 16/3227, S.21.

688 Bei einem titulierten Forderungsattribut ist wiederum § 184 II InsO einschlägig.

689 Vgl. Begr. zu Nr.23 (§ 184 InsO) RegE, BT-Drucks. 16/3227, S.21.

690 So auch Kübler/Prütting/Bork/Pape, § 184 Rn.29. Wobei der widersprechende Insolvenzverwalter im Gegensatz zum widersprechenden Insolvenzgläubiger grundsätzlich schon von Amts wegen gehalten ist, zeitnah Feststellungsklage zu erheben. Dennoch würde eine solche Ausschlussfrist willkürliche Widersprüche vorbeugen, die dann doch nicht weiterverfolgt würden.
} 
widerspruchsbetroffenen Insolvenzgläubiger bleibt in diesem Fall in aller Regel genügend Zeit sich zu überlegen, ob er gegen den Widerspruch vorgehen will oder nicht. Dem Schuldner verschafft diese Anknüpfung hinsichtlich dieser Insolvenzforderungen Gewissheit über seine Nachhaftung, wodurch ihm die Entscheidung erleichtert wird, ob er seinen Restschuldbefreiungsantrag zurückzieht oder nicht. ${ }^{691}$

Für Insolvenzforderungen, die im Schlusstermin mit dem Forderungsattribut angemeldet werden bzw. erst geprüft werden können, ${ }^{692}$ wird es für den Schuldner bezüglich dieser Forderungen jedoch bei einer nicht auszuschließenden Ungewissheit bleiben, da in diesem Fall ein eindeutiger und damit handhabbarer Anknüpfungspunkt aus der derzeitigen Gesetzeslage nicht ersichtlich ist. Auch wenn so verspätete Anmeldungen von Insolvenzforderungen aus vorsätzlich begangener unerlaubter Handlung in der Praxis die Ausnahme und nicht die Regel darstellen, ist insbesondere für diese Insolvenzforderungen ein durch den Gesetzgeber zu regelnder strenger zeitlicher Maßstab wünschenswert. ${ }^{693}$ Dem Insolvenzgläubiger sind ja die grundsätzliche Anmeldungsmöglichkeit seiner Forderung und deren Tragweite für den Schuldner hinsichtlich § 302 Nr.1 InsO hinreichend und frühzeitig bekannt. Einziger Wermutstropfen für den Schuldner wird in diesem Fall die Möglichkeit der Verjährungseinrede gegen das Forderungsattribut sein. Denn im Gegensatz zur fortbestehenden Verjährungshemmung nach Beendigung des Insolvenzverfahrens bei beantragter Restschuldbefreiung für (eventuell

691 Die Rücknahmemöglichkeit des Restschuldbefreiungsantrages bis zur Ankündigung der Restschuldbefreiung ist allgemein anerkannt. Vgl. nur FK/Ahrens, § 287 Rn.15 m.w.N.

692 Dies gilt natürlich entsprechend auch für eine angesetzte Prüfung im schriftlichen Verfahren.

693 Für diesbezügliche Insolvenzforderungen wäre in § 184 I InsO - neben einer zu normierenden Ausschlussfrist für die bereits im Vorfeld des Schlusstermins geprüften und vom Schuldner widersprochenen Insolvenzforderungen, die - wie gezeigt - an den Schlusstermins zu knüpfen sein wird - im Gegensatz zu der einmonatigen Ausschlussfrist in $\S 184$ II InsO (die auch in $\S 179$ II InsO umgesetzt werden sollte) eine verkürzte zweiwöchige Ausschlussfrist ab Widerspruchserhebung [wegen der in diesem Fall wohl anzusetzenden Prüfung im schriftlichen Verfahren; siehe dazu unter E.II.3.a) (S.192ff.)] anzudenken, die mit einer entsprechend befristeten - wohl in $\S 289$ InsO zu normierenden - Rücknahmemöglichkeit des Schuldners hinsichtlich seines Antrages auf Restschuldbefreiung zu koppeln sein wird. Für eine so zeitnah an die Ankündigung der Restschuldbefreiung gelegene Rücknahmemöglichkeit des Antrages sollten keine Bedenken bestehen. Vgl. dazu auch den derzeitigen Meinungsstand hinsichtlich der Rücknahmemöglichkeit des Restschuldbefreiungsantrags während der fortgeschrittenen Treuhandperiode etwa bei FK/Ahrens, § 287 Rn.16. 
daneben bestehende) vertragliche/gesetzliche Anspruchsgrundlage der Insolvenzforderung gilt dies nicht für den Rechtgrund einer Insolvenzforderung aus vorsätzlich begangener unerlaubter Handlung, ${ }^{694}$ so dass nach der sechsmonatigen Nachfrist (§ 204 II 1 BGB) die dreijährige Verjährungsfrist (§ 195 BGB) für diesen Rechtsgrund weiterläuft. Insofern wird zumindest in dieser Hinsicht der Insolvenzgläubiger gehalten sein, zu seiner eigenen Rechtssicherheit innerhalb der sechsmonatigen Nachfrist Feststellungsklage zu erheben.

Bei einem Schuldnerwiderspruch in einem Insolvenzverfahren ohne Antrag auf Restschuldbefreiung wird derzeit hingegen von keiner zeitlichen Beschränkung der Erhebung einer Feststellungsklage gem. $\S 184$ I InsO ausgegangen werden können, da ein frühzeitiges Klärungsinteresse des Schuldners im Sinne der Intention des Gesetzgebers in diesem Fall fehlt. ${ }^{695}$

694 Siehe bereits unter D.VI.4. (S.115ff.). Vgl. auch Kübler/Prütting/Bork/Pape, § 184 Rn.117f.

695 Der zeitliche Rahmen für eine Feststellungsklage wird für den Insolvenzgläubiger in diesem Fall durch die Verjährungsfrist seiner Forderung nach Beendigung des Insolvenzverfahrens bestimmt sein. Zudem wird die Wirkung des $\S 89$ InsO mit aufgehoben. Siehe zur Beendigung der Hemmung auch unter D.VI.4. (S.115ff.). 


\section{Nachträgliche Anmeldungen}

Im Folgenden soll nun auf die Möglichkeit der nachträglichen Anmeldung von Insolvenzforderungen im Insolvenzverfahren eingegangen werden. $\S 177$ InsO stellt klar, dass die im Eröffnungsbeschluss vom Insolvenzgericht nach $\S 28 \mathrm{I}$ InsO zu bestimmende Anmeldefrist keine Ausschlussfrist ist, ${ }^{696}$ und regelt das Verfahren hinsichtlich der Prüfung von nachträglich angemeldeten Insolvenzforderungen, also solchen Insolvenzforderungen, die nach Ablauf der vom Insolvenzgericht festgelegten Anmeldefrist beim Insolvenzverwalter angebracht werden (§ 177 I 1 InsO). Weiterhin werden die Prüfung von zur Anmeldung zugelassenen nachrangigen Insolvenzforderungen (§ 177 II InsO) und die Behandlung von nachträglichen Änderungen der Anmeldung (§ 177 I 3 InsO) geregelt. ${ }^{697}$ Die Norm dient der Verfahrensökonomie indem einerseits der Prüfungstermin nicht durch verspätete Anmeldungen aufgehalten wird, andererseits aber die Säumigen ihre Rechte gleichwohl sachgerecht wahren können.

So wird das Interesse des Insolvenzgläubigers, auch noch nachträglich mit seiner Forderung am Insolvenzverfahren teilzunehmen, den Prüfungs- und Widerspruchsrechten der bereits formell teilnehmenden Insolvenzgläubiger in den einzelnen Verfahrensstadien gegenüberzustellen, Probleme aufzuzeigen und diese einer jeweils ausgeglichenen Interessenlösung zuzuführen sein. Dabei wird nach (1.) Besprechung der verfahrenstechnischen Behandlung von nachträglichen Anmeldungen zunächst (2.) auf die Ausübung und Folge des Mitprüfungswiderspruchs der formellen Verfahrensbeteiligten einzugehen sein, um daraufhin (3.) der Frage nachzugehen, bis wann eine Forderung überhaupt zum Insolvenzverfahren anmeldbar sein muss, um die Interessen und Verfahrensrechte des Insolvenzgläubigers zu wahren, aber auch, welche Einschnitte er je nach Anmeldezeitpunkt diesbezüglich u.U. hinnehmen muss.

\footnotetext{
696 Siehe bereits unter D.II. (S.30ff.); vgl. auch Begr. zu § 204 (§ 177 InsO) RegE, BT-Drucks. 12/2443, S.184

697 Bereits unter D.IV.7. (S.72ff.).
} 


\section{Anmeldung und Eintragung verspäteter Insolvenzforderungen}

Sämtliche Insolvenzforderungen und damit auch die nachträglichen Insolvenzforderungen sind beim Insolvenzverwalter anzumelden und von ihm auf ihre formelle Zulässigkeit zu prüfen. ${ }^{698}$ Bis zur Niederlegung der Tabelle auf der Geschäftsstelle des Insolvenzgerichts muss der Insolvenzverwalter auch die nachträglich angemeldeten Insolvenzforderungen mit in die Tabelle eintragen, so dass diese unproblematisch mit den übrigen fristgerecht angemeldeten Anmeldungen und der Tabelle beim Insolvenzgericht zur Einsicht und Prüfung niedergelegt werden können und das Insolvenzgericht seiner eventuell bestehenden Hinweispflicht gem. § 175 II InsO nachkommen kann. Die Verspätung der eingetragenen Anmeldung ergibt sich grundsätzlich aus dem Eingangsvermerk, kann aber auch zusätzlich in der Tabelle vermerkt werden. Nach Niederlegung der Tabelle geht die Tabellenführung auf das Insolvenzgericht über, so dass der Insolvenzverwalter sämtliche nach diesem Zeitpunkt bei ihm eingehenden nachträglichen Anmeldungen zwar gleichwohl auf ihre formelle Zulässigkeit prüft, die Nachtragung der soweit formell ordnungsgemäßen - Anmeldungen in die Tabelle bei gleichzeitiger Einreichung aller übrigen Unterlagen jedoch vom Insolvenzgericht vornehmen lässt. Hierdurch wird sichergestellt, dass die übrigen Verfahrensbeteiligten schnellstmöglich von weiteren Anmeldungen Kenntnis nehmen, diese vor dem Prüfungstermin nach Möglichkeit noch ausreichend prüfen können und zudem keine Unregelmäßigkeiten in der inhaltlichen Aussage der Tabelle entstehen. ${ }^{699}$ Weiterhin ist für das Insolvenzgericht dadurch frühzeitig absehbar, ob es verfahrenstechnisch günstiger ist, anstatt eines besonderen Prüfungstermins ein schriftliches Verfahren anzuordnen.

698 Siehe bereits unter D.IV.5.d) (S.65ff.).

699 Siehe bereits unter D.V.3. (S.87ff.). 


\section{Insolvenzforderungsanmeldung bis zum Ende des Prüfungstermins}

$\mathrm{Zu}$ unterscheiden ist zwischen der verfahrensmäßigen Behandlung von Insolvenzforderungen, die nach Ablauf der Anmeldefrist, aber vor dem Prüfungstermin angemeldet werden, und solchen, deren Anmeldung erst nach dem Prüfungstermin erfolgt. Im Prüfungstermin sind gem. § 177 I 1 InsO grundsätzlich auch diejenigen Insolvenzforderungen mitzuprüfen, die zwischen dem Ablauf der Anmeldefrist und dem Ende des Prüfungstermins angemeldet worden sind, soweit der Insolvenzverwalter (Sachwalter/Treuhänder) oder ein widerspruchsberechtigter Insolvenzgläubiger der Prüfung der verspäteten Anmeldungen im Prüfungstermin nicht widerspricht (vgl. § 177 I 2 1.Alt. InsO). Den Verfahrenbeteiligten muss nämlich grundsätzlich die materiellrechtliche Prüfung der einzelnen angemeldeten Insolvenzforderungen (§ 175 I 2 InsO) ermöglicht werden. Sollte ihnen dies aufgrund der verspäteten Anmeldung jedoch nicht ausreichend möglich gewesen sein, so können sie der sofortigen Mitprüfung widersprechen.

Aus dem Gesetzeswortlaut ergibt sich, dass sich der hier gemeinte Widerspruch ausschließlich auf die Zulassung der verspätet angemeldeten Insolvenzforderung zur Mitprüfung im regulären Prüfungstermin bezieht, und nicht (auch) auf das allgemeine Widerspruchsrecht gegen die materiellrechtliche Berechtigung der angemeldeten Insolvenzforderung zur Teilnahme am Verfahren. ${ }^{700}$ Der Widerspruch richtet sich dabei gegen die Mitprüfung aller bis dahin angebrachten nachträglichen Insolvenzforderungen insgesamt. Etwas anderes lässt sich dem Wortlaut des § 177 I InsO nicht entnehmen - der nur von den „Forderungen“ (§ 177 I 1 InsO) und „dieser Prüfung“ (§ 177 I 2 InsO) spricht - und dient der Gläubigergleichbehandlung (z.B. hinsichtlich der Kostenlast eines besonderen Prüfungstermins, § 177 I 2 InsO). ${ }^{701}$ Außerdem würde einem isolierten Mitprüfungswiderspruch gegenüber jeder einzelnen verspäteten Anmeldung im Verfahren mehr Bedeutung zukommen und dieser insoweit auch den Fortgang des Prüfungstermins beeinträchtigen, als der eigentlichen Prüfung der Insolvenzforderung im Prüfungstermin selbst

\footnotetext{
700 Graf-Schlicker/Graf-Schlicker, § 177 Rn.2; MüKo-InsO/Nowak, § 177 Rn.3; BK/Breutigam/Erckens, § 177 Rn.5.

701 Siehe auch unter E.II.4. (S.198ff.).
} 
zusteht. $^{702}$ Der Widerspruch gegen eine Mitprüfung ist dabei ebenfalls mündlich und ohne Begründung im Prüfungstermin zu erheben, ${ }^{703}$ da die objektive Tatsache der Verspätung ausreichend ist. Sollte der Insolvenzverwalter oder einer der Insolvenzgläubiger der sofortigen Mitprüfung im regulären Prüfungstermin widersprechen, so hat das Insolvenzgericht auf Kosten des/der Säumigen zwingend einen besonderen Prüfungstermin zu bestimmen, oder die Prüfung im schriftlichen Verfahren anzuordnen (§ 177 I 2 2.Halbsatz InsO).

Der Schuldner hat diese Widerspruchsbefugnis hingegen nicht, da insoweit der Insolvenzverwalter die Prüfung an seiner Statt vornimmt und der Mitprüfung bei Bedarf widersprechen würde. Nur bei der Eigenverwaltung kann auch der Schuldner der sofortigen Mitprüfung in seiner Eigenschaft als Eigenverwalter widersprechen. ${ }^{704}$ Allerdings wäre zu überlegen, ob dem Schuldner hinsichtlich der Mitprüfung von Insolvenzforderungen aus vorsätzlich begangener unerlaubter Handlung nicht ein Widerspruchsrecht einzuräumen ist, um eine ausreichende eigene Prüfung der Insolvenzforderung $\mathrm{zu}$ ermöglichen, weil der Insolvenzverwalter diesbezüglich gerade keine Prüfungs- und Widerspruchspflicht hat ${ }^{705}$ und der Mitprüfung - wenn die Insolvenzforderung ansonsten unproblematisch sofort prüfbar und teilnahmeberechtigt ist - regelmäßig nicht widersprechen würde. Jedoch hat das Insolvenzgericht bei allen nachträglichen Anmeldungen darauf $\mathrm{zu}$ achten, dass es seiner Hinweispflicht gem. § 175 II InsO gegenüber dem Schuldner hinsichtlich solcher Insolvenzforderungen aus vorsätzlich begangener unerlaubter Handlung ausreichend nachkommen kann. ${ }^{706}$ Sollte dies nicht der Fall sein, so hat das Insolvenzgericht die nachträglich angemeldete(n) Insolvenzforderung(en) im regulären Prüfungstermin von Amts wegen nicht zur Prüfung zu stellen, sondern einen besonderen Prüfungstermin oder ein schriftliches Verfahren anzusetzen. Insofern wird dem Schuldner regelmäßig durch das Insolvenzgericht im Rahmen seiner Hinweispflicht ausreichend Zeit zur Prüfung zur Verfügung gestellt, so dass ihm in

\footnotetext{
Siehe bereits unter E.I.3. (S.137ff.).

Bereits unter E.I.4.c)aa) (S.144ff.).

704 Siehe bereits unter D.VI.2.b) (S.102ff.).

705 Siehe bereits unter E.I.4.c)cc) (S.148ff.).

706 Bereits unter D.V.5. (S.90ff.).
} 
Übereinstimmung mit $\S 177$ I 2 InsO kein Widerspruchsrecht gegen die Mitprüfung zuzusprechen ist.

§ 177 I 1 InsO knüpft die Widerspruchsbefugnis gegen die Mitprüfung der verspätet angemeldeten Insolvenzforderung allein an die Versäumung der vom Insolvenzgericht eindeutig festgelegten Anmeldefrist. Dieses Widerspruchsrecht steht dem Insolvenzverwalter und den formellen Insolvenzgläubigern uneingeschränkt zu. ${ }^{707}$ Die Prüfung der Anmeldung hinsichtlich der Aufnahme der Insolvenzforderung in die Tabelle durch den Insolvenzverwalters bezieht sich nämlich zunächst nur auf die formelle Zulässigkeit der Anmeldung zum Verfahren, ${ }^{708}$ sei sie fristgerecht oder nachträglich angebracht worden, und bedeutet nicht, dass bereits auch eine umfassende und abschließende materiellrechtliche Prüfung der Insolvenzforderung selbst stattgefunden hat. Das Gesetz knüpft den Mitprüfungswiderspruch an die abgelaufene Anmeldefrist und nicht an die Niederlegung der Tabelle an, so dass dem Insolvenzverwalter und den Insolvenzgläubigern auch gegen die Mitprüfung von Insolvenzforderungen, die trotz ihrer Verspätung noch Aufnahme in die Tabelle bis zu ihrer Niederlegung beim Insolvenzgericht gefunden haben, ein Widerspruchsrecht zuzubilligen ist. Täte man dies nicht, sondern ginge man von einem Widerspruchsverbot gegen die Mitprüfung dieser bereits aufgenommenen nachträglichen Anmeldungen aus, weil trotz der verspäteten Anmeldung die Mindestzeit zur Vorbereitung auf den Prüfungstermin verbliebe, ${ }^{709}$ so wäre ein solches Widerspruchsverbot die Versagung eines Insolvenzgläubigerrechts aufgrund eines (bloß verfahrensökonomischen) Verhaltens des Insolvenzverwalters, was mit der eindeutigen gesetzlich festgelegten Regelung in § 177 InsO und vor allem mit der Gläubigerautonomie im Insolvenzverfahren ${ }^{710}$ nicht zu vereinbaren ist. Das einheitlich ab Ablauf der Anmeldefrist geltende Widerspruchsrecht würde außerdem bei Zulassung einer solchen Verfahrensweise auseinanderfallen bzw. zu einer Privilegierung der bereits bei Niederlegung in die Tabelle aufgenommenen nachträglichen Anmeldungen

\footnotetext{
707 Graf-Schlicker/Graf-Schlicker, $\S 177$ Rn.2; Gottwald/Eickmann, $\S 63$ Rn.50; Uhlenbruck/Uhlenbruck, § 177 Rn.3.

708 Bereits unter D.IV.5.a) (S.53ff.).

709 So MüKo-InsO/Nowak, § 177 Rn.3; Nerlich/Römermann/Becker, § 177 Rn.9; FK/Kießner, § 177 Rn.6.

710 Siehe bereits unter B. (S.4ff.).
} 
führen. Dies ist weder mit der Wirkung des Mitprüfungswiderspruchs gegen alle nachträglichen Anmeldungen - etwa bei einem erfolgten Mitprüfungswiderspruch aufgrund noch nach Niederlegung der Tabelle eingegangener Anmeldungen - noch mit der allen Insolvenzgläubigern verspäteter Anmeldungen zu gleichen Teilen aufzuerlegenden Kostenlast in Einklang zu bringen, die für den dann vom Insolvenzgericht anzuberaumenden zusätzlichen Prüfungstermin bzw. das schriftliche Prüfungsverfahren ${ }^{711}$ anfallen. ${ }^{712}$ Die Ansicht vom Verbot des Mitprüfungswiderspruchs wäre nur dann mit Regelung und Wirkung des § 177 I InsO vereinbar, wenn das Widerspruchsrecht an die Niederlegung der Tabelle anknüpfen würde. Das bestimmt § 177 I 1 InsO aber gerade nicht.

\section{Insolvenzforderungsanmeldung nach dem Prüfungstermin}

\section{a) Letzte Anmelde- und Prüfungsmöglichkeit}

Geht eine nachträgliche Forderungsanmeldung erst ein, nachdem der reguläre Prüfungstermin stattgefunden hat, so muss das Insolvenzgericht grundsätzlich entweder einen besonderen Prüfungstermin oder die Prüfung der Insolvenzforderung im schriftlichen Verfahren anordnen (vgl. § 177 I 2 Alt.2 InsO). Die Entscheidung, welche der Möglichkeiten das Insolvenzgericht für die Durchführung des besonderen Prüfungstermins wählt, steht dabei im freien Ermessen des verfahrensleitenden Insolvenzgerichts. Es wird bei seiner Entscheidung abzuwägen haben, mit welcher Möglichkeit das Insolvenzgericht Entlastungen für das Verfahren erzielen kann. ${ }^{713}$

Das Gesetz regelt dabei nicht, bis wann eine Forderungsanmeldung überhaupt möglich ist. Daher könnte man annehmen, dass eine Anmeldung bis zur Aufhebung oder Beendigung des Insolvenzverfahrens möglich ist. Jedoch bedarf die nachträgliche Anmeldung einer Insolvenzforderung immer auch ihrer Prüfung, um festgestellt werden zu können. Die letzte

\footnotetext{
Siehe unter E.II.4. und 5. (S.198ff., 200ff.).

Dies widerspräche dem Gläubigergleichbehandlungsgrundsatz.

713 MüKo-InsO/Ganter, $\S 5$ Rn.64a; HHK/Preß/Henningsmeier, $\S 177$ Rn.9; Braun/Specovius, § 177 Rn.8.
} 
Möglichkeit einer solchen Prüfung stellt dabei der Schlusstermin als die in § 197 InsO festgelegte abschließende Gläubigerversammlung des Insolvenzverfahrens dar. ${ }^{714}$ Durch $\S 87$ InsO besteht ausschließlich im Anmelde- und Feststellungsverfahren der §§ 174ff. InsO die Möglichkeit, Insolvenzforderungen verfolgen zu können, so dass der Insolvenzgläubiger grundsätzlich ein rechtliches Interesse daran hat, dass er - neben der verjährungshemmenden Wirkung der ordnungsgemäßen Anmeldung ${ }^{715}$ seine Insolvenzforderung bis in die letzte Phase des Verfahrens durch ihre Prüfung und Feststellung titulieren (§§ 178 I, III, 201 II 1 InsO) lassen ${ }^{716}$ und auch alle sonstigen mit der formellen Insolvenzgläubigerstellung verbundenen Verfahrensrechte des Insolvenzverfahrens erlangen kann. Daher muss die Anmeldung und Feststellung einer Insolvenzforderung grundsätzlich bis zum Ende des Schlusstermins möglich sein. Auf die Terminierung eines weiteren Prüfungstermins nach Abhaltung des Schlusstermins kann aufgrund der eindeutigen Regelung des § 197 InsO hingegen kein Anspruch und auch keine Möglichkeit bestehen, ${ }^{717}$ da das Insolvenzverfahren durch weitere Prüfungstermine ansonsten endlos in die Länge gezogen werden könnte. ${ }^{718}$ Der Schlusstermin ist zwar in der Regel nicht zugleich ${ }^{719}$ auch besonderer Prüfungstermin; doch auch er ist eine Gläubigerversammlung, in der zumindest der Insolvenzverwalter anwesend ist, der die verspätete Anmeldung wie regelmäßig im gesamten Insolvenzverfahren auch im Interesse der eventuell nicht anwesenden übrigen Verfahrensteilnehmer aufnehmen und prüfen kann. ${ }^{720}$ Insofern kann mangels weiterer ansetzbarer Gläubigerversammlungen auch ein Widerspruch gegen die Mitprüfung gem. § 177 I 2 InsO der erst im Schlusstermin angebrachten Insolvenzforderung nur zur Folge haben, dass insbesondere bei Insolvenzforderungen aus vorsätzlich begangener unerlaubter Handlung aufgrund der Hinweispflicht des Insolvenzgerichts

\footnotetext{
714 BGH ZIP 1998, 515 (515); MüKo-InsO/Nowak, § 177 Rn.4; Uhlenbruck/Uhlenbruck, $\S 177$ Rn.2; Gerbers/Pape, ZInsO 2006, 685 (686); Braun/Specovius, § 177 Rn.3 m.w.N.

715 Dazu bereits unter D.VI.4. (S.115ff.).

716 BGH ZIP $1998 \quad 515 \quad$ (515f.); $\quad$ MüKo-InsO/Nowak, $\S 177 \quad$ Rn.4; Kübler/Prütting/Bork/Pape, § 177 Rn.2; Gerbers/Pape, ZInsO 2006, 685 (687); Uhlenbruck/Uhlenbruck, § 177 Rn.2 m.w.N.

717 Kübler/Prütting/Bork/Pape, § 177 Rn.2; Uhlenbruck/Uhlenbruck, § 177 Rn.2; Braun/Specovius, § 177 Rn.3; Gerbers/Pape, ZInsO 2006, 685 (686).

718 Siehe dazu auch folgend unter E.II.3.b) (S.194ff.).

719 Siehe aber E.II.4. (S.198ff.).

720 Für den Schuldner wird in diesem Fall § 186 InsO entsprechend anzuwenden sein.
} 
gegenüber dem Schuldner (vgl. § 175 II InsO) ${ }^{721}$ vom Insolvenzgericht eine Forderungsprüfung im schriftlichen Verfahren anzusetzen ist. ${ }^{722}$ Eine vereinzelt noch im Schlusstermin angebrachte Insolvenzforderung wird jedoch die Ausnahme bleiben, so dass ein Widerspruch gegen ihre Mitprüfung mit der Konsequenz der Einleitung eines schriftlichen Verfahrens - solange es sich nicht um eine Insolvenzforderung mit Forderungsattribut handelt - regelmäßig verfahrensunökonomisch sein wird. Vielmehr wird der Insolvenzverwalter in diesem Fall, wenn eine unproblematische Prüfung der Insolvenzforderung im Schlusstermin nicht sofort möglich sein sollte, regelmäßig auf den „vorläufigen“ Widerspruch zurückgreifen, um nach seiner Prüfung den Widerspruch unproblematisch zurücknehmen zu können. ${ }^{723}$

\section{b) Aufnahme der Insolvenzforderung ins Schlussverzeichnis}

Hiervon abzugrenzen ist jedoch die Frage, ob eine so verspätet angemeldete und vielleicht erst im Schlusstermin geprüfte und festgestellte Insolvenzforderung neben der Eintragung in die Tabelle auch noch in das Schlussverzeichnis aufgenommen werden darf, um auch zur Teilnahme an der Schlussverteilung und eventuellen Nachtragsverteilungen, die gem. $\S 205$ S.1 InsO ebenfalls auf dem Schlussverzeichnis beruhen, berechtigt zu sein. ${ }^{724}$ Hier ist nämlich grundsätzlich zwischen den beiden Verfahrensabschnitten (1.) der Anmeldung und Feststellung der Insolvenzforderungen ( $\S \S 174$ bis 186 InsO) und (2.) der anschließenden Verteilung (§§ 187 bis 206 InsO) zu unterscheiden, wobei sich beide überlappen können, insbesondere wenn nachträgliche Anmeldungen die

Siehe bereits unter D.V.5. (S.90ff.).

Dies wohl in der Form einer ,schriftlich fortzuführenden Vertagung des Schlusstermins“ i.S.d. § 177 I 2, III 3 InsO allein hinsichtlich der Prüfung der verspäteten Insolvenzforderung, wodurch die öffentliche Bekanntmachung entfällt und keine missverständliche Außenwirkung hinsichtlich einer weiterhin andauernden Anmeldungsmöglichkeit von Insolvenzforderungen entstehen kann. Hinsichtlich der Ladungen gem. § 177 III 2 InsO siehe folgend unter E.II.3.b) (S.194ff.).

723 Siehe bereits unter E.I.4.d)bb) (S.160ff.).

724 Die Aufnahme der Insolvenzforderung in das Schlussverzeichnis ist darüber hinaus für die Antragsrechte des Insolvenzgläubigers gem. §§ 290, 296, 297 InsO hinsichtlich der Versagung einer Restschuldbefreiung von Bedeutung (vgl. § 292 I 2 InsO). Siehe hierzu BGH ZInsO 2005, 597ff.; Ahrens, NZI 2005, 401ff.; Pape, ZInsO 2005, 599f. 
Anberaumung eines weiteren (besonderen) Prüfungstermins erforderlich machen (vgl. § 177 II InsO).

Der Insolvenzverwalter hat gem. § 188 InsO für die Schlussverteilung (§ 196 InsO) ein auf der Insolvenztabelle beruhendes ${ }^{725}$ Verteilungsverzeichnis zu erstellen, das sogenannte Schlussverzeichnis (vgl. § 205 S.1 InsO). Demgemäß dürfen auch nur bis zum Schlusstermin angemeldete, geprüfte, festgestellte und damit zur Teilnahme am Insolvenzverfahren berechtigte Insolvenzforderungen oder zumindest gem. § 189 I InsO berücksichtigungsfähige Insolvenzforderungen aus der Tabelle ins Schlussverzeichnis übernommen werden. ${ }^{726}$ Insofern ist zwischen der Eintragung der (verspäteten) Insolvenzforderung in die Tabelle und ihrer Berücksichtigung im Schlussverzeichnis streng zu unterscheiden. Nach Niederlegung und Veröffentlichung des Schlussverzeichnisses (§ 188 InsO) können Änderungen des Schlussverzeichnisses nur aufgrund der Regelungen der $\S \S 189$ bis 193 InsO oder aber zur Berichtigung offensichtlicher Irrtümer oder Unrichtigkeiten vorgenommen werden. ${ }^{727}$ Diese Änderungen, die aufgrund der §§ 189 bis $192 \mathrm{InsO}^{728}$ erforderlich werden, hat der Insolvenzverwalter gem. § 193 InsO binnen drei Tagen nach Ablauf der in $\S 189$ I InsO vorgesehenen Ausschlussfrist vorzunehmen. Zeitlich darüber hinaus eröffnen die Bestimmungen der §§ 189 bis 193 InsO aber keine Änderungsmöglichkeit des Schlussverzeichnisses, etwa hinsichtlich einer nachträglichen und erst im Schlusstermin geprüften und in die Tabelle aufgenommenen Insolvenzforderung. Die Insolvenzgläubiger müssen zum Zeitpunkt der Niederlegung und Veröffentlichung die Sicherheit haben, wie das Schlussverzeichnis abschließend aussieht, denn nur dann können sie entscheiden, ob sie am Schlusstermin teilnehmen und gegen das

725 KO-Motive, S.374 = Hahn, KO, S.335; MüKo-InsO/Füchsl/Weishäupl, § 188 Rn.3 m.w.N.

726 Die notwendige Anmeldung (§ 174 InsO) und Prüfung (§§ 176, 177 InsO) der Insolvenzforderung zur Aufnahme in das Verteilungsverzeichnis ergibt sich schon aus § 187 I InsO, der festlegt, dass eine Verteilung erst nach dem Prüfungstermin begonnen werden darf.

727 BGH NZI 2007, 401 (402).

$728 \S 192$ InsO ist aufgrund seines eindeutigen Wortlautes nur auf Abschlagsverteilungen und nicht auch auf die Schlussverteilung anwendbar. Handelt es sich bei der anstehenden Verteilung nämlich (lediglich) um eine Abschlagsverteilung, kann der Gläubiger gem. § 192 InsO an einer späteren Verteilung teilnehmen und dabei vorrangig berücksichtigt werden, wenn er bis dahin den Nachweis der §§ 189, 190 InsO geführt oder gar im Feststellungsverfahren obsiegt hat. 
Schlussverzeichnis gem. $\S 197$ I Nr.2 InsO Einwendungen erheben wollen. ${ }^{729}$ Lässt der Insolvenzgläubiger ordnungsgemäß gesetzte und bekannt gemachte Anmeldungsfristen im Anschluss an den allgemeinen Prüfungstermin ungenutzt verstreichen, kann er folglich nicht damit rechnen, dass seine noch nach dem besonderen Prüfungstermin angemeldete Insolvenzforderung Aufnahme in das Schlussverzeichnis findet. Auch wenn nachträgliche Anmeldungen bis zum Schlusstermin möglich sind, muss es eine zeitliche Begrenzung für die Teilnahme an der Verteilung der Insolvenzmasse geben. Eine unbegrenzte Möglichkeit nachträglicher Insolvenzforderungsanmeldungen ohne Ausschluss von der Verteilung würde sonst dazu führen, dass kaum ein Insolvenzverfahren abgeschlossen werden kann. ${ }^{730}$ Einer solchen Lösung würden zudem insbesondere die Gläubigerinteressen entgegenstehen, die auf eine möglichst hohe Befriedigung des Einzelnen, aber auch auf eine zeitnahe Befriedigung der Gemeinschaft ausgerichtet sind. Im Übrigen zeigt auch die Regelung des § 206 InsO, dass Forderungsverluste der Gläubiger von der Insolvenzordnung durchaus hingenommen werden, wenn es um den Fortgang des Verfahrens geht. Daher kann nur eine bis zur Niederlegung und Veröffentlichung des Schlussverzeichnisses angemeldete und geprüfte Insolvenzforderung Aufnahme in das Schlussverzeichnis finden und an der Schluss- und eventuellen Nachtragsverteilungen teilnehmen. ${ }^{731}$

Ist eine untitulierte Forderungsanmeldung zum Zeitpunkt der Veröffentlichung der Schlussverteilung bereits angemeldet und geprüft, aber bestritten worden, besteht für den Gläubiger bezogen auf seine Teilhabe an der Insolvenzmasse hingegen vollwertiger Rechtsschutz. Der Insolvenzgläubiger muss dafür gem. § 189 I InsO lediglich innerhalb einer Ausschlussfrist von zwei Wochen nach der öffentlichen Bekanntmachung des Schlussverzeichnisses dem Insolvenzverwalter nachweisen, dass und für welchen Betrag die Feststellungsklage erhoben oder das Verfahren in dem früher anhängigen Rechtsstreit aufgenommen ist. Folge des rechtzeitigen Nachweises ist gem. $§ \quad 189$ II InsO, dass der

BGH NZI 2007, 401 (402); MüKoInsO-Nowak, § 177 Rn.4.

Gerbers/Pape, ZInsO 2006, 685 (688); Gundlach/Frenzel, NZI 2007, 402 (402).

BGH NZI 2007, 401 (402); Braun/Kießner, § 189 Rn.16; Gerbers/Pape, ZInsO 2006, 685 (688); siehe weiterhin OLG Köln ZIP 1994, 949 (949f.); HHK/Preß, § 188 Rn.4; MüKo-InsO/Füchsl/Wieshäupl, § 189 Rn.12; BK/Breutigam/Erckens, $\S 177$ Rn.9; MüKo-InsO/Nowak, § 177 Rn.4; KS/Eckardt, S.759 Rn.31. 
Insolvenzgläubiger die bestrittene Insolvenzforderung in das Schlussverzeichnis aufnehmen muss, die entsprechende Quotenauszahlung aber bis zu einer rechtskräftigen Entscheidung des Feststellungsrechtsstreites zurückzuhalten ist. Wird dieser Nachweis hingegen nicht rechtzeitig geführt, so findet auch eine geprüfte, aber bestrittene Insolvenzforderung keine Berücksichtigung und damit auch keine Aufnahme in das Schlussverzeichnis; sie bleibt bei der Schlussverteilung der Insolvenzmasse (vgl. § 189 III InsO) und auch bei den eventuellen Nachtragsverteilungen unberücksichtigt.

Eine bestrittene titulierte Insolvenzforderung ist hingegen in jedem Fall ins Schlussverzeichnis aufzunehmen (Umkehrschluss aus § 189 I InsO). Hier wird jedoch § 189 II InsO, trotz seines Wortlautes, entsprechend auch auf titulierte Insolvenzforderungen anzuwenden sein, wenn Feststellungsklage von dem Bestreitenden erhoben wurde und dem Insolvenzverwalter nachgewiesen ist, so dass auch in diesem Fall der auf die Insolvenzforderung entfallende Anteil bei der Verteilung zurückbehalten wird, solange der Rechtsstreit anhängig ist. Denn nach dem Willen des Gesetzgebers sollten die Vorschriften der $\S \S 152,168$ Nr.1 KO sinngemäß übernommen werden. ${ }^{732} \S 168 \mathrm{Nr} .1 \mathrm{KO}$ ordnete die Zurückbehaltung auch für bestrittene, wenngleich titulierte Forderungen an, sofern diese infolge eines bei der Prüfung erhobenen Widerspruchs im Prozess befangen sind. Wie bei bestrittenen nicht titulierten Forderungen, bei denen der Feststellungsprozess betrieben wird, darf dem ungewissen Ausgang des anhängigen Feststellungsprozesses durch das Verteilungsverfahren nicht vorgegriffen werden. Wenn trotz erhobener Feststellungsklage des Bestreitenden und ohne die Entscheidung hierüber abzuwarten, gleichwohl auf die betroffene Insolvenzforderung eine Auszahlung erfolgen würde, wäre die Verteilungsgerechtigkeit gefährdet und das Widerspruchsrecht gegen bereits titulierte Forderungen faktisch entwertet. ${ }^{733}$ Dies will der Insolvenzgläubiger und/oder Insolvenzverwalter durch seinen Widerspruch und den rechtzeitigen Nachweis der Weiterverfolgung seines Widerspruchs durch das Erheben der Feststellungsklage gerade verhindern. Daher ist auch im Fall einer titulierten, aber bestrittenen Insolvenzforderung § 189 II

732 Begr. zu § 217 (§ 189 InsO) RegE, BT-Drucks. 12/2443, S.186.

733 MüKoInsO-Füchsl/Wieshäupl, § 189 Rn.10. 
InsO analog anzuwenden und der entsprechende Anteil nicht auszubezahlen, sondern zurückzubehalten, solange das Ergebnis des Feststellungsrechtsstreites aussteht. ${ }^{734}$ Denn anders als bei $\S 189$ I InsO geht es in § 189 II InsO nicht um das „ob“, sondern um das „wie“ der Berücksichtigung. Der Nachweis der Klageerhebung kann daher auch bis zum Vollzug der Verteilung geführt werden. ${ }^{735}$

\section{Der besondere Prüfungstermin}

Der besondere Prüfungstermin ist wie der reguläre Prüfungstermin eine Gläubigerversammlung, so dass alle bisherigen Ausführungen dazu auch für den besonderen Prüfungstermin gelten. Er ist immer dann erforderlich, wenn der Mitprüfung nachträglicher Anmeldungen gem. § 177 I 2 InsO im (regulären) Prüfungstermin widersprochen wurde und/oder auch noch nach Beendigung des Prüfungstermins verspätete Anmeldungen beim Insolvenzverwalter angebracht werden. Die Ergänzung der in diesem Stadium des Verfahrens bereits niedergelegten Insolvenztabelle durch die nachträglichen Anmeldungen erfolgt nun jedoch ausschließlich durch das Insolvenzgericht, nachdem der Insolvenzverwalter die bei ihm anzubringenden Anmeldungen auf ihre formelle Zulässigkeit geprüft hat. ${ }^{736}$ Das Gesetz sieht keine Frist für den besonderen Prüfungstermin vor, sondern verlangt nur, dass der Termin öffentlich bekannt zu machen ist und der Insolvenzverwalter, der Schuldner und (lediglich) die nachträglichen Insolvenzgläubiger zu dem Termin zu laden sind (§ 177 III 1, 2 InsO). ${ }^{737}$ Anderenfalls wäre die zusätzliche Anordnung der öffentlichen Bekanntmachung des $\S 177$ III 1 InsO zur sonstigen

734 Nerlich/Römermann/Westphal, § 189 Rn.18; MüKo-InsO/Füchsl/Wieshäupl, § 189 Rn.10; Holzer, NZI 1999, 44 (45f.); Smid/Smid, § 189 Rn.6; Braun/Kießner, § 189 Rn.13 m.w.N.

735 MüKo-InsO/Füchsl/Wieshäupl, § 189 Rn.10; siehe auch Uhlenbruck/Uhlenbruck, $\S 189$ Rn.8: Braun/Kießner, § 189 Rn.13.

736 Siehe bereits unter E.II.1. (S.188f.).

737 Kübler/Prütting/Bork/Pape, § 177 Rn.4; Uhlenbruck/Uhlenbruck, § 177 Rn.8; FK/Kießner, § 177 Rn.9f.; Gottwald/Eickmann, § 63 Rn.53; KS/Eckardt, S.760 Rn.33. 
allgemeinen Information überflüssig. ${ }^{738}$ Das Insolvenzgericht wird sich jedoch hinsichtlich seines freien Ermessens zur Bestimmung des besonderen Termins an der Frist des $\S 29$ I Nr.2 InsO (mindestens eine Woche) und dem allgemeinen Fortgang des Verfahrens zu orientieren haben. ${ }^{739}$ Auf die öffentliche Bekanntmachung kann aber auch hier verzichtet werden, ${ }^{740}$ wenn im regulären Prüfungstermin bereits ein besonderer Prüfungstermin vor Ort angeordnet und festgelegt werden kann, weil gegen die sofortige Mitprüfung der bis dahin eingegangenen nachträglichen Anmeldungen Widerspruch erhoben wurde (§ 177 III 3 i.V.m. $§ 74$ II 2 InsO). ${ }^{741}$ Mit der Bekanntgabe des besonderen Prüfungstermins, gleich auf welcher Weise, sollte das Insolvenzgericht auf die unveränderte Prüfungsmöglichkeit der ausgelegten nachträglichen Anmeldungen auf der Geschäftsstelle des Insolvenzgerichts hinweisen. Die Kosten für den besonderen Prüfungstermin oder das schriftliche Verfahren $^{742}$ sind gem. § 177 I 2 InsO vom säumigen Insolvenzgläubiger zu tragen ${ }^{733}$ und werden ausschließlich durch die verspätete Anmeldung - gleich aus welchem Grund - veranlasst und sind dem Säumigen damit verschuldensunabhängig aufzuerlegen. ${ }^{744}$

Die Verbindung des besonderen Prüfungstermins mit dem Schlusstermin verbietet die Insolvenzordnung nicht; sie ist daher grundsätzlich möglich. ${ }^{745}$ Von dieser Möglichkeit sollte jedoch nur dann Gebrauch gemacht werden, wenn eine Ladung zu einem (weiteren) besonderen Prüfungstermin oder die Anordnung zur Prüfung in einem schriftlichen Verfahren nicht mehr rechtzeitig vor dem bereits festgelegten

738 Die öffentliche Bekanntmachung ersetzt die Zustellung dabei nur hinsichtlich der in der öffentlichen Bekanntmachung erscheinenden Inhalte, so dass sich die persönliche Ladung nicht etwa gem. § 9 III InsO erübrigt. Wollte das Insolvenzgericht die Ladung mit in die Bekanntmachung einrücken, läge regelmäßig ein unzumutbarer Eingriff in das Recht auf informationelle Selbstbestimmung der Verfahrensbeteiligten vor. - Nerlich/Römermann/Becker, § 177 Rn.39.

739 Siehe bereits unter D.III. (S.33ff.) und D.IV.7. (S.72ff.).

740 Siehe bereits unter E.II.2. (S.189ff.).

741 Begr. des Rechtsausschusses zu § 177 InsO, BT-Drucks. 14/120, S.13.

742 Falls für das schriftliche Verfahren überhaupt (Verfahrens-)Kosten anzunehmen sind.

743 Dies bemisst sich nach dem Kostenverzeichnis in Anlage 1 zum Gerichtskostengesetz (GKG) vom 5. Mai 2004 (BGBl. I S. 718) und unter Umständen nach §§ 3ff. der Insolvenzrechtliche Vergütungsverordnung (InsVV) vom 19. August 1998 (BGBl. I S. 2205).

744 Kübler/Prütting/Bork/Pape, § 177 Rn.7; Uhlenbruck/Uhlenbruck, § 177 Rn.9; Nerlich/Römermann/Becker, § 177 Rn.18; MüKo-InsO/Nowak, § 177 Rn.10 m.w.N.

745 Vgl. Begr. zu § 201 (§ 174 InsO) RegE, BT-Drucks. 12/2443, S.184; BGH NZI 2007, 401 (402); Uhlenbruck/Uhlenbruck, § 177 Rn.5; MüKo-InsO/Nowak, § 177 Rn.6; Kübler/Prütting/Bork/Pape, § 177 Rn.4 m.w.N. 
Schlusstermin ergehen kann, da durch die Prüfung dieser verspäteten Anmeldung erst im Schlusstermin die Insolvenzforderung nicht mehr ins Schlussverzeichnis zur Teilnahme an der Schlussverteilung aufgenommen werden kann. ${ }^{746}$ Der säumige Insolvenzgläubiger, der seine Insolvenzforderung nach Ablauf der allgemeinen Frist des § 28 I InsO anmeldet, kann zwar darauf vertrauen, dass das Gericht alle im Rahmen des laufenden Verfahrens bestehenden Möglichkeiten ausschöpft, um die Verspätung aufzufangen, er hat aber keinen Anspruch darauf, dass der Verfahrensverlauf seinetwegen angehalten wird, indem das Insolvenzgericht den Schlusstermin aufhebt und vertagt. ${ }^{777}$ Eine Vertagung beruht hier nämlich auf dem Versäumnis des Insolvenzgläubigers und ist daher nicht mit einer möglichen Vertagung des allgemeinen Prüfungstermins, die wegen zu vieler Insolvenz-forderungen auf einer nicht möglichen Prüfung aller fristgerecht angemeldeten Insolvenzforderungen innerhalb eines Prüfungstermins beruht, vergleichbar. ${ }^{748}$

\section{Das schriftliche Verfahren}

Neben der Möglichkeit eines schriftlichen Prüfungsverfahrens im Rahmen der nachträglichen Anmeldung gem. § 177 InsO kann das Insolvenzgericht gem. § 5 II InsO nunmehr auch generell anordnen, dass das gesamte Insolvenzverfahren oder einzelne seiner Teile schriftlich durchgeführt werden, wenn die Vermögensverhältnisse des Schuldners überschaubar und die Zahl der Gläubiger oder die Höhe der Verbindlichkeiten gering sind. An diesen Vorgaben des § 5 II InsO wird sich das Insolvenzgericht auch hinsichtlich seiner Ermessensentscheidung für ein schriftliches Prüfungsverfahren - sowohl von Anfang an, als auch im Rahmen des $\S 177$ InsO - zu orientieren haben, wenn es nämlich das Insolvenzgericht entlastet. Ansonsten ist die Möglichkeit des unmittelbar mündlichen

746 Bereits unter E.II.3.b) (S.194ff.). Siehe auch MüKo-InsO/Nowak, § 177 Rn.6; Uhlenbruck/Uhlenbruck, § 177 Rn.5; Gerbers/Pape, ZInsO 2006, 685 (686); Haarmeyer/Wutzke/Förster, Kap.7 Rn.66; wohl auch BGH NZI 2007, 401 (402).

747 Gerbers/Pape, ZInsO 2006, 685 (686). Siehe hinsichtlich der Möglichkeiten bei einer Anmeldung im Schlusstermin unter E.II.3.b) (S.194ff.).

748 Siehe bereits unter E.I.4.d)bb) (S.160ff.). 
Austausches der Verfahrensbeteiligten in einem Prüfungstermin hinsichtlich einer zügigen Verfahrensdurchführung der Vorzug zu geben. ${ }^{749}$ Das Insolvenzgericht kann diese Anordnung jederzeit aufheben oder abändern, wobei die Anordnung, ihre Aufhebung oder Abänderung zusammen mit Terminen oder Fristen öffentlich bekannt zu machen sind (vgl. §§ 5 II 2, 3; 177 III 1 InsO).

Die Ausgestaltung des schriftlichen Prüfungsverfahrens ist in der Insolvenzordnung nicht weiter geregelt. Daher werden die Ladungs- und Bekanntmachungsregelungen der $\S \S 74$ II 1, 177 III InsO weitgehend entsprechend anzuwenden sein. So wird das Insolvenzgericht neben der öffentlichen Bekanntmachung und der persönlichen Ladung im Fall des § 177 III 1, 2 InsO je nach Verfahrensstand das schriftliche Prüfungsverfahren auch schon im Eröffnungsbeschluss oder entsprechend $\S 74$ II 2 InsO (vgl. § 177 III 3 InsO) in einer Gläubigerversammlung ohne zusätzliche öffentliche Bekanntmachung anordnen können. ${ }^{750}$

Das schriftliche Verfahren ersetzt im Rahmen der §§ 174ff. InsO nur die sonst nötigen Gläubigerversammlungen, so dass sich hinsichtlich der grundsätzlichen Forderungsanmeldung, Tabellenerstellung, Niederlegung, Tabelleneinsicht und -fortführung keine Veränderungen gegenüber dem allgemeinen Verfahren ergeben. Den Verfahrensbeteiligten Kopien/Abschriften der angemeldeten Insolvenzforderung mit der Bitte zur Stellungnahme als Alternative zur Auslage in der Geschäftsstelle zukommen zu lassen, ${ }^{751}$ erfordert regelmäßig - sei es seitens des Insolvenzverwalters im Fall des $\S 8$ III InsO oder gerade im fortgeschrittenen Verfahren seitens des Insolvenzgerichts (Geschäftsstelle) - einen erhöhten Kosten- und Verwaltungsaufwand, der durch das schriftliche Verfahren aber gerade vermieden werden soll. Zudem müssten für eine ordentliche Prüfungsmöglichkeit den Verfahrensbeteiligten auch sämtliche mit der Anmeldung eingereichten Unterlagen als Kopie/Abschrift überlassen werden, was jedoch hinsichtlich der Wahrung und Kontrolle des Akteneinsichtrechts und der damit verbundenen Interessen der

749 Begr. Rechtsausschuss zu § 204 (§ 177 InsO), BT-Drucks. 12/7302, S.179 und Begr. zu $\S 5$ InsO RegE, BR-Drucks. 549/06, S.21; siehe auch Schmerbach, ZInsO 2002, 292 (292f.).

750 Vgl. auch Nerlich/Römermann/Becker, § 177 Rn.40.

751 So Hess, InsO, § 177 Rn.25 (selbst wohl widersprüchlich, vgl. Rn.24); wohl auch BK/Breutigam/Erckens, § 177 Rn.11; Gottwald/Eickmann, § 63 Rn.60. 
Verfahrensbeteiligten, insbesondere des Schuldners, ${ }^{752}$ äußerst bedenklich ist. Daher ist diese Alternative insgesamt abzulehnen.

Der entscheidende Unterschied zum regulären liegt hinsichtlich des schriftlichen Prüfungsverfahren nur in Form und Frist, indem das Insolvenzgericht eine Ausschlussfrist - entsprechend dem ansonsten zu bestimmenden (besonderen) Prüfungstermin - zu bestimmen hat, bis zu der die Widerspruchsberechtigten ihr Widerspruchsrecht schriftlich ausüben können. Die Erhebung des Widerspruchs ist in diesem Fall nur bis zum Ende dieser Ausschlussfrist - wie er sonst auch nur wirksam im Prüfungstermin möglich ist ${ }^{753}$ - und nur schriftlich oder zu Protokoll der Geschäftsstelle (§ 496 ZPO i.V.m. $§ \quad 4$ InsO) gegenüber dem Insolvenzgericht möglich, da die Tabelleneintragung wie im Prüfungstermin entsprechend $\S 178$ II InsO vom Insolvenzgericht vorzunehmen ist. $^{754}$ Die Wiedereinsetzung in den vorigen Stand würde auch bei Fristversäumnis entsprechend § 186 I InsO nur dem Schuldner zustehen, ${ }^{755}$ wobei ein Versäumnis durch die zu erfolgende „Ladung“ zur schriftlichen Prüfung und Bekanntgabe der Ausschlussfrist regelmäßig so gut wie ausgeschlossen ist. Damit unnötige Anfragen beim Insolvenzgericht hinsichtlich der Prüfungsergebnisse vermieden werden, ist das Prüfungsergebnis - wie sonst auch - nur denjenigen Insolvenzgläubigern vom Insolvenzgericht mitzuteilen, deren angemeldete Forderungen bestritten worden sind (§ 179 III InsO). ${ }^{756}$

Siehe bereits unter D.VI.3. (S.108ff.).

753 Siehe bereits unter E.I.4.b) (S.142ff.) und E.I.4.c)aa) (S.144ff.).

754 HK/Irschlinger, § $177 \quad$ Rn.8; Uhlenbruck/Uhlenbruck, $\S 177$ Rn.10; BK/Breutigam/Erckens, § 177 Rn.11; MüKo-InsO/Nowak, § 177 Rn.8; KS/Eckardt, S.760 Rn.33; Nerlich/Römermann/Becker, § 177 Rn.15ff.; Graf-Schlicker/GrafSchlicker, § 177 Rn.19.

755 Siehe bereits unter E.I.4.b) (S.142ff.).

756 Das Insolvenzgericht weißt im Eröffnungsbeschluss darauf hin, dass Insolvenzgläubiger unbestrittener und damit festgestellter Forderungen nicht benachrichtigt werden. - Vgl. Begr. zu § 207 (§ 179 III InsO) RegE, BT-Drucks. 12/7302, S.179. 


\section{Nachrangige Insolvenzforderungen}

Nachrangige Insolvenzgläubiger ( $§ 39$ InsO) nehmen am Verfahren nur teil, wenn sie zur Anmeldung ihrer Forderungen ausdrücklich aufgefordert wurden ( $§ 174$ III 1 InsO). Aufgrund ihrer Nachrangigkeit können diese Insolvenzforderungen aber nur dann zur Anmeldung aufgefordert werden, wenn die Forderungen der nicht-nachrangigen Insolvenzgläubiger zu 100\% befriedigt werden können. ${ }^{757}$ Praktische Bedeutung hat dies daher eigentlich nur in Verfahren, in denen saniert und nicht liquidiert werden soll. ${ }^{758}$

Hat das Insolvenzgericht die nachrangigen Insolvenzgläubiger in Anwendung des § 174 III 1 InsO erst so spät zur Anmeldung ihrer Forderungen zur Tabelle aufgefordert, dass die ihnen gesetzte Anmeldefrist später als eine Woche vor dem allgemeinen Prüfungstermin abläuft, ${ }^{759}$ so werden diese angemeldeten nachrangigen Insolvenzforderungen in $\S 177$ II InsO ohne weiteres wie verspätete Anmeldungen nach $\S 177$ I 2 InsO behandelt, selbst wenn niemand ihrer Mitprüfung im regulären Prüfungstermin widersprochen hat. Die verspäteten Anmeldungen und damit die Notwendigkeit der Vornahme der Prüfung dieser nachträglichen Insolvenzforderungen in einem besonderen Prüfungstermin, sind in diesem Fall nicht auf ein Versäumnis der nachrangigen Insolvenzgläubiger zurückzuführen, da sie bislang überhaupt keine Möglichkeit hatten, ihre Insolvenzforderungen zum Verfahren anzumelden. ${ }^{760}$ Deshalb bestimmt $\S 177$ II InsO, dass in diesen Fällen die Kosten des besonderen Prüfungstermins bzw. die des schriftlichen Prüfungsverfahrens die Insolvenzmasse $\mathrm{zu}$ tragen hat. Dies hat entsprechend zu gelten, wenn die Aufforderung zur Anmeldung der nachrangigen Insolvenzforderungen erst nach bereits abgehaltenem regulärem Prüfungstermin erfolgt. Werden die nachrangigen Insolvenzgläubiger in Gruppen ( $§ 39$ I Nr.1-5 InsO) zur Anmeldung

757 MüKo-InsO/Nowak, § 177 Rn.5; Graf-Schlicker/Graf-Schlicker, § 177 Rn.14; Smid, Grundzüge, § 2 Rn.23f.

758 Siehe bereits unter C.III. (S.20ff.).

759 Der Wochenabstand ist Grenzwert in Parallele zum regulären Prüfungstermin gem. $\S 29$ I Nr.2 2.Halbsatz InsO.

760 Die Regelung passt nur deshalb unter die Überschrift „nachträgliche Anmeldungen“, weil aus der Sicht der nicht-nachrangigen Insolvenzgläubiger die Anmeldung nachrangiger Insolvenzforderungen mit Verspätung erfolgt. 
aufgefordert, gilt das zu den Anmeldefristen Gesagte für den jeweils vorangehenden Prüfungstermin. ${ }^{761}$

Liegt das Ende der Anmeldefrist vor der letzten Woche vor dem Prüfungstermin, werden die angemeldeten nachrangigen Insolvenzforderungen im eigentlichen Prüfungstermin geprüft. Versäumt der nachrangige Insolvenzgläubiger seine Anmeldefrist, so fällt seine Anmeldung nicht mehr unter $\S 177$ II InsO, sondern gilt als allgemein verspätet, und § 177 I InsO findet wiederum Anwendung. ${ }^{762}$

Im Übrigen wird das Insolvenzgericht hinsichtlich der Aufnahme der nachrangigen Insolvenzforderungen in das Schlussverzeichnis gehalten sein, die nachrangigen Insolvenzgläubiger so zu ihrer Anmeldung aufzufordern, dass ihre Prüfung vor der Erstellung und Niederlegung des Schlussverzeichnisses in einem besonderen Prüfungstermin oder einem schriftlichen Verfahren stattfinden kann.

\section{Rechtsmittel}

Sowohl bei erhobenem Widerspruch gegen die Mitprüfung als auch bei Anmeldung nach dem Prüfungstermin hat das Insolvenzgericht zwingend einen besonderen Prüfungstermin oder das schriftliche Prüfungsverfahren anzuordnen. Wird die betreffende nachträgliche Anmeldung dennoch im allgemeinen Prüfungstermin zur Prüfung gestellt, bestand bei Entscheidungen durch den Insolvenzrichter - was die seltene Ausnahme darstellt - bisher nach § 73 III KO die Möglichkeit, sofortige Beschwerde einzulegen, was nunmehr durch § 6 I InsO ausgeschlossen ist. Beruht die Prüfung im Termin allerdings auf einer Entscheidung des Rechtspflegers, kann die sofortige Erinnerung nach § 11 II 1 RPflG eingelegt werden. Hilft der Rechtspfleger der Erinnerung nicht ab, entscheidet der Insolvenzrichter gem. § 11 II 3 RPflG abschließend.

Wenn das Insolvenzgericht hingegen trotz nachträglicher Anmeldungen weder einen nachträglichen Prüfungstermin festsetzt, noch die Prüfung im schriftlichen Verfahren verfügt, so steht dem betreffenden

761 Nerlich/Römermann/Becker, § 177 Rn.26; MüKo-InsO/Nowak, § 177 Rn.5.

762 Graf-Schlicker/Graf-Schlicker, § 177 Rn.14; Uhlenbruck/Uhlenbruck, § 177 Rn.13; vgl. auch Nerlich/Römermann/Becker, § 177 Rn.34ff. 
Insolvenzgläubiger nur die Möglichkeit offen, Antrag an das Insolvenzgericht auf Tätigwerden zu stellen, da es sich hierbei lediglich um eine verfahrensleitende Maßnahme handelt, gegen die ein Rechtsmittel nicht gegeben ist. ${ }^{763}$ 


\section{Die Feststellung der Insolvenzforderungen im Prüfungstermin}

Die Vorschrift des $\S 178$ InsO regelt die Voraussetzungen und Wirkungen der unstreitigen Feststellung ordnungsgemäß angemeldeter Insolvenzforderungen. Die Feststellung der Insolvenzforderung ist Voraussetzung für die Teilnahme an der insolvenzrechtlichen Befriedigung des Gläubigers (§§ 189 I 1, 187 InsO). Sie ermöglicht nach Beendigung des Insolvenzverfahrens und der sich eventuell anschließenden Wohlverhaltensphase die Vollstreckung aus dem Tabelleneintrag gegen den Schuldner persönlich (§§ 201 II 1, 294 I InsO). Ferner sichert die Feststellung das Stimm- und Widerspruchsrecht bei Gläubigerversammlungen. ${ }^{764}$

Im Folgenden sollen daher (1.) die Voraussetzungen und der Umfang einer möglichen Feststellung der ordnungsgemäß angemeldeten Insolvenzforderung zur Tabelle aufgezeigt und erörtert sowie (2.) der Zeitpunkt und die Wirkung und Tragweite der Feststellung auf die Insolvenzforderung selbst und für das Insolvenzverfahren und darüber hinaus für die nachinsolvenzliche Haftung des Schuldners besprochen werden. Weiterhin wird (3.) zu klären sein, was genau Gegenstand der Feststellung ist und inwiefern daraus Rückschlüsse auf (4.) eine mögliche Feststellung von Nichtinsolvenzforderungen gezogen werden können. Schließlich wird (5.) das Verhältnis zwischen der nachinsolvenzlichen Erteilung eines vollstreckbaren Tabellenauszuges und einem bereits bei Eröffnung des Insolvenzverfahrens bestehenden Titel $\mathrm{zu}$ erörtern und die damit verbundenen nachinsolvenzlichen Interessen der Insolvenzgläubiger denen des Schuldners gegenüberzustellen sein, um unter Ausschöpfung der verfahrensrechtlichen Vorgaben eine einheitliche und ausgeglichene Interessenlösung aufzeigen zu können.

764 Siehe bereits unter D.VI. (S.94ff.) und E.I.4.f)bb) (S.170ff.). 


\section{Voraussetzung der Feststellung}

Gem. § 178 I 1 InsO gilt die ordnungsgemäß angemeldete und geprüfte Insolvenzforderung als festgestellt, soweit weder der Insolvenzverwalter noch ein formeller Insolvenzgläubiger im Prüfungstermin oder im schriftlichen Prüfungsverfahren widerspricht oder soweit ein erhobener Widerspruch beseitigt worden ist. ${ }^{765}$ Eine ausdrückliche Zustimmung der widerspruchsberechtigten Verfahrensbeteiligten sieht das Gesetz somit nicht vor, da die Feststellung unstreitiger Insolvenzforderungen nach dem Willen des Gesetzgebers zügig und einfach erfolgen soll. ${ }^{766}$ Das Nichtbestreiten wirkt als stillschweigendes Anerkenntnis. ${ }^{767}$ Daher ist auch die Anwesenheit des einzelnen Insolvenzgläubigers im Prüfungstermin für die Feststellung einer angemeldeten Forderung nicht nötig. ${ }^{768}$

Durch den Wortlaut "soweit" in § 178 I 1 InsO wird deutlich, dass auch eine Teilfeststellung grundsätzlich möglich ist. Dies ist jedoch nur dann der Fall, wenn sich der Widerspruch allein gegen die Höhe der angemeldeten Insolvenzforderung richtet, oder wenn der Widersprechende die Nachrangigkeit der Insolvenzforderung (§ 39 InsO) behauptet und das Insolvenzgericht zur Anmeldung von Insolvenzforderungen des anerkannten Nachranges aufgefordert hat. ${ }^{769}$ In beiden Fällen bleibt es nämlich unbestritten, dass die angemeldete Insolvenzforderung zur Teilnahme am Verfahren und damit zur Teilhabe an der Insolvenzmasse grundsätzlich befugt ist. Gleiches hat auch für den isolierten Widerspruch eines Insolvenzgläubigers gegen das Forderungsattribut der vorsätzlich begangenen unerlaubten Handlung zu gelten. ${ }^{770}$

Siehe bereits ab E.I.4. (S.140ff.).

KO-Motive, S.357 = Hahn, KO, S.322.

767 RGZ 55, 157 (160); HHK/Preß/Henningsmeier, $\S 178$ Rn.6; MüKoInsO/Schumacher, § 178 Rn.2; Graf-Schlicker/Graf-Schlicker, § 178 Rn.2.

768 Vgl. KO-Motive, S.362 = Hahn, KO, 325; siehe auch bereits unter E.I.2.a) (S.135).

769 KS/Eckardt, S.756 Rn.26; MüKo-InsO/Schumacher, § 178 Rn.10; Graf-

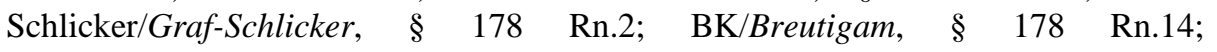
HHK/Preß/Henningsmeier, § 178 Rn.7.

770 Siehe bereits unter E.I.4.c)cc) (S.148ff.). 


\section{Rechtskraftwirkung des feststellenden Tabellenvermerks}

Gemäß § 178 III InsO wirkt die Eintragung in die Tabelle für festgestellte Insolvenzforderungen ihrem Betrag und ihrem Rang nach wie ein rechtskräftiges Urteil gegenüber dem Insolvenzverwalter und allen Insolvenzgläubigern. Durch die rein beurkundende Tätigkeit des Insolvenzgerichts liegt hier zwar gerade keine Urteilsentscheidung im Sinne einer der $\S \S 300 f f$. ZPO vor, ${ }^{771}$ gleichwohl bewirkt die Eintragung in die Tabelle nach den zu § 322 ZPO entwickelten Grundsätzen in gleichem Umfang Rechtskraftwirkung zwischen den Parteien wie ein rechtskräftiges Urteil. ${ }^{772}$ Die Erstreckung der subjektiven Rechtskraftwirkung des Feststellungsvermerks - über den Personenkreis des § 325 I ZPO hinaus auch auf das Verhältnis des Insolvenzgläubigers der festgestellten Insolvenzforderung $\mathrm{zu}$ allen übrigen Insolvenzgläubigern ${ }^{773}$ und dem Insolvenzverwalter hat ihre rechtfertigende Grundlage in der Selbstverwaltungskompetenz der Gläubigergesamtheit hinsichtlich der ihr haftungsrechtlich zugewiesenen Insolvenzmasse und in der Besonderheit der Insolvenz als ein Gesamtvollstreckungsverfahren. Gleiches bestimmt $\S 183$ I InsO für die im Feststellungsstreit gem. §§ 179ff. InsO errungene Zuerkennung. Das mit der Anmeldung erhobene „Begehren“ auf Feststellung der Teilnahmeberechtigung am Insolvenzverfahren richtet sich hier nämlich nicht nur gegen den Insolvenzverwalter als Träger der Insolvenzmasse ( $\S 80$ I InsO), sondern auch gegen alle übrigen mitkonkurrierenden Insolvenzgläubiger, sei es dass sie ihre Forderungen zum Insolvenzverfahren angemeldet haben oder nicht. Dies ergibt sich aus dem Widerspruchsrecht gem. § 178 I 1 i.V.m. § 176 InsO, welches allen Insolvenzgläubigern bei Anmeldung ihrer eigenen Insolvenzforderung gegen die übrigen angemeldeten Insolvenzforderungen eingeräumt ist. ${ }^{74}$ Durch die generelle Verfahrensbetroffenheit aller Insolvenzgläubiger im Sinne des $\S 38$ InsO und deren damit verbundenen prinzipiellen

771 Kein Anerkennungsurteil, vgl. RGZ 37, 386 (388).

772 BGH ZInsO 2005, 372 (373); KS/Eckardt, S.763 Rn.38; Uhlenbruck/Uhlenbruck, $\S 178$ Rn.12; MüKo-InsO/Schumacher, § 178 Rn.59 m.w.N.

773 Insolvenzgläubiger i.S.d. § 178 III InsO können nach Zweck der Vorschrift nur Inhaber anderer Insolvenzforderungen sein, nicht diejenigen, welche die festgestellte Insolvenzforderung für sich selbst in Anspruch nehmen („Prätendentenstreit“). - RGZ 58, 369 (372ff.); BGH NJW 1997, 1014 (1015); Jaeger/Weber, KO, § 145

Rn.8; MüKo-InsO/Schumacher, § 178 Rn.68 m.w.N.

774 Siehe bereits unter D.VI.1. (S.94ff.). 
Anmeldungs- und Mitwirkungsmöglichkeit am Prüfungsverfahren muss sich dann aber auch die Rechtskraftwirkung des Feststellungsvermerks, der gerade auf ihrer gemeinsamen und gegenseitigen Annerkennungsmöglichkeit beruht, ${ }^{775}$ auf alle Insolvenzgläubiger erstrecken, ${ }^{776}$ und dies eben unabhängig von ihrer tatsächlichen Anmeldung (vgl. § 302 I 2 InsO) und über das Insolvenzverfahren hinaus. ${ }^{777}$ Denn die Insolvenzforderungen sind in einem rechtsförmlichen Verfahren unter Gewährung des rechtlichen Gehörs für alle Insolvenzgläubiger aufgrund pflichtgemäßer Prüfung durch den Insolvenzverwalter und des unterlassenen - aber durch Teilnahme am Verfahren eben grundsätzlich möglichen - Widerspruchs der Insolvenzgläubiger festgestellt worden. Könnten solche Insolvenzforderungen in einem Rechtsstreit - gleich ob von einem formellen oder, aus welchen Gründen auch immer, lediglich verfahrensbetroffen gebliebenen Insolvenzgläubiger - nochmals nach Beendigung des Insolvenzverfahrens bestritten werden, müsste praktisch die Forderungsfeststellung des Insolvenzverfahrens wiederholt werden, was dem Sinn und Zweck der einheitlichen Feststellung gegenüber allen (verfahrensbetroffenen) Insolvenzgläubigern widerspräche. Eine solche mehrfach rechtsförmlige Prüfung ein und desselben Anspruchs ist mit einer ökonomischen Rechtsgewährung nicht $\mathrm{zu}$ vereinbaren. ${ }^{778}$ Einwendungen gegen die zur Insolvenztabelle festgestellten Insolvenzforderungen können insofern nur noch mit Rechtsmitteln geltend gemacht werden, die gegen rechtkräftige Urteile zulässig sind. ${ }^{779}$ Gleiches gilt daher selbstverständlich auch hinsichtlich des eigenständigen Widerspruchsrechts des Insolvenzverwalters, wobei die Rechtskraftwirkung gegenüber dem Insolvenzverwalter zugleich die Wirkung gegenüber dem Schuldner bedeutet, soweit sein Vermögen dem Insolvenzverfahren unterliegt. Denn der Schuldner muss nunmehr hinnehmen, dass aus dem Erlös der Verwertung seines Vermögens der

\footnotetext{
775 KO-Motive, S.362 = Hahn, KO, S.325: „Die Theilnahme der einzelnen Gläubiger ...ist...naturgemäß eine freiwillige; sie können erscheinen und sich erklären, brauchen es aber nicht; ihr Schweigen gilt...als Zustimmung zu den Erklärungen des Verwalters.“

776 Jaeger/Weber, KO, § 145 Rn.5 m.w.N.

777 Allgm. Meinung: RGZ 37, 1 (2); MO-Motive, S.364 = Hahn, KO, S.327; MüKoInsO/Schumacher, § 178 Rn. 67 m.w.N. Siehe bereits unter D.I. (S.23ff.).

778 BGH KTS 1984, 602 (606) m.w.N.

779 Siehe dazu unter E.III.7. (S.234f.).
} 
Insolvenzforderungsinhaber Befriedigung erhält, so als ob der Insolvenzgläubiger aus einem rechtskräftigen Urteil die Zwangsvollstreckung betriebe. ${ }^{780}$ Aus $\S 201$ II i.V.m. § 178 III InsO folgt zudem, dass der Feststellungsvermerk auch gegenüber dem Schuldner persönlich und über das Insolvenzverfahren hinaus Rechtskraftwirkung erzeugen kann, wenn der Schuldner der Feststellung nicht widersprochen hat oder sein Widerspruch nicht beseitigt wurde. ${ }^{781}$ Diese Regelungen sollen die abschließende Prüfung der Insolvenzforderungen im Prüfungstermin bzw. generell im Insolvenzverfahren gewährleisten. Einwendungen gegen angemeldete Insolvenzforderungen sollen im Prüfungstermin vorgebracht werden, widrigenfalls sie auf Dauer durch die rechtskräftige Feststellung der Insolvenzforderung ausgeschlossen sind. ${ }^{782}$ Hierdurch soll der „Unzulänglichkeit nachträglicher Widersprüche“ und unnötiger Feststellungsklagen begegnet und sowohl „für alle späteren Verhandlungen im Konkurse, als auch...über den Konkurs hinaus...eine judikatsmäßig feste...Grundlage gewonnen“ werden. ${ }^{783}$

Die ausdrücklich gem. §§ 178 I, 183 I InsO nach Sinn und Zweck des Insolvenzverfahrens lediglich auf alle Insolvenzgläubiger, den Insolvenzverwalter und - bei unterlassenem Schuldnerwiderspruch - auch auf den Schuldner persönlich erweiterte subjektive Rechtskraftwirkung (§§ 322, 325 I ZPO) der Tabellenfeststellung erstreckt sich daher grundsätzlich nicht auf die Rechte Dritter, ${ }^{784}$ da diese hinsichtlich ihrer Rechte aufgrund der fehlenden Insolvenzgläubigerstellung im Sinne des $\S 38$ InsO gerade nicht Verfahrensbetroffene sind und daher auch nicht am Insolvenzverfahren hätten mitwirken können. Dritten gegenüber wirkt die

780 MüKo-InsO/Schumacher, § 178 Rn.70; Nerlich/Römermann/Becker, § 178 Rn.24.

781 RGZ 112, 297 (300); BGH WM 1961, 427 (429); OLG Köln WM 1995, 597 (599); BGH ZInsO 2005, 372 (372f.); KS/Eckardt, S.763f. Rn.39; MüKo-InsO/Schumacher, § 178 Rn.70; Uhlenbruck/Uhlenbruck, § 178 Rn.12; Hess, InsO, § 178 Rn.29; Nerlich/Römermann/Becker, § 178 Rn.24; Kübler/Prütting/Bork/Pape, § 178 Rn.6; Häsemeyer, InsO, Rn.22.03, 22.20.

782 KO-Motive, S.357, 364 = Hahn, KO, S.322, 327; RGZ 57, 270 (274); vgl. auch BGH ZIP 1984, 1509ff.; ZIP 1987 725ff.; Kübler/Prütting/Bork/Pape, § 178 Rn.8; MüKoInsO/Schumacher, § 178 Rn.59.

783 So bereits ausdrücklich KO-Motive, S.357, 364 = Hahn, KO S.322, 327.

784 RGZ 46, 8 (10); Hess, InsO, § 178 Rn.30; Uhlenbruck/Uhlenbruck, § 178 Rn.18; MüKo-InsO/Schumacher, § 178 Rn.72; Kübler/Prütting/Bork/Pape, § 178, Rn.6f.; Kuhn/Uhlenbruck, KO, § 145 Rn.6; Jaeger/Weber, KO, § 145 Rn.5.

Die Feststellung hat keine Auswirkungen auf Bürgen, Zedenten oder Gläubigern von Sicherheitsrechte: vgl. etwa BGH ZIP 1995, 2161 (2161); BGH WM 1974, 1218 (1218); RGZ 51, 33 (51); Kübler/Prütting/Bork/Pape, § 178 Rn.7 m.w.N. 
Rechtskraft grundsätzlich nur nach Maßgabe der allgemein geltenden materiell- und prozessrechtlichen Bestimmungen. ${ }^{785}$ Hiervon zu unterscheiden sind die insolvenzverfahrensrechtlich bedingten Vollstreckungsbeschränkungen des § 89 InsO, denen teilweise auch Dritte bzw. konkret die Neugläubiger, deren Forderungen erst nach Eröffnung des Insolvenzverfahrens entstanden sind, unterliegen, wodurch der Bestand des haftenden Vermögens während des Verfahrens gesichert werden soll. Gläubiger, die keine Insolvenzgläubiger sind, unterfallen gerade nicht den insolvenzverfahrensrechtlichen Vorschriften (§§ 87, 38 i.V.m. $\S \S 174 f f$. InsO) und können dementsprechend während des Insolvenzverfahrens ihre Forderungen nach den allgemeinen zivilprozessualen Grundsätzen geltend machen, jedoch steht ihnen zur Vollstreckung hinsichtlich der Durchsetzung ihrer Forderungen für die Dauer des Insolvenzverfahrens nur das insolvenzfreie Vermögen des Schuldners zur Verfügung (vgl. § 36 I InsO), da die mit Eröffnung des Insolvenzverfahrens unter Beschlag genommene Insolvenzmasse ausschließlich den Insolvenzgläubigern vorbehalten ist (§§ 35, 38 InsO). Zusätzlich bestimmt § 89 II InsO, dass während der Verfahrensdauer Zwangsvollstreckungen in künftige Bezüge aus einem Dienstverhältnis ausdrücklich verwehrt sind, es sei denn es handelt sich um einen Unterhaltsanspruch oder eine neue Forderung aus einer vorsätzlich begangenen unerlaubten Handlung, die in den erweitert pfändbaren Teil der Bezüge vollstreckt werden können (§§ 850d, 850f II ZPO). ${ }^{786}$ Ausgenommen dieser Ausnahme in Satz 2 soll mithilfe des § 89 II InsO der Schuldner in den Stand gesetzt werden, nach Verfahrensbeendigung seine pfändbaren Forderungen auf Bezüge aus einem Dienstverhältnis zum Zwecke der Restschuldbefreiung an einen Treuhänder abzutreten (vgl. § 287 II InsO). ${ }^{787}$ Jedoch wird wegen ihrer

785 RGZ 46, 8 (10); Kuhn/Uhlenbruck, KO, § 145 Rn.6. Vereinzelte Ausnahmeentscheidungen der Praxis, in denen sich die rechtkräftige Feststellung der Insolvenzforderung (un-)mittelbar auch auf die Rechtsverhältnisse Dritter auswirkt, sind stets zu beachten, begründen aber keine gegenteilige Annahme zum gegebenen Grundsatz. Siehe etwa BGH ZIP 1990, 175 (175) zur Feststellung der sich aus der vollzogenen Wandlung des Leasingvertrags ergebenden Forderung zur Tabelle. Weitere Einzelheiten mit Entscheidungsverweisen siehe bei MüKoInsO/Schumacher, § 178 Rn.73

786 Dieser nicht zur Insolvenzmasse gehörende Teil der Bezüge wird von der die Restschuldbefreiung bezweckende Abtretung der (pfändbaren) Bezüge an den Treuhänder nicht erfasst und unterliegt darum dem Zugriff der privilegierten Neugläubiger. Vgl. Begr. zu § 100 (§ 89 InsO) RegE, BT-Drucks. 12/2443, S.137f.

787 BGH ZInsO 2008, 39 (40); FK/App, § 89 Rn.13; MüKo-InsO/Breuer, § 89 Rn.35. 
besonderen Schutzbedürftigkeit das Vollstreckungsverbot zugunsten der Neugläubiger, die im Insolvenzverfahren gerade nicht berücksichtigt werden und infolge der Einbeziehung des Neuerwerbs in die Insolvenzmasse (vgl. § 35 InsO) keinen realistischen Vollstreckungszugriff auf das insolvenzfreie Vermögen haben, im Umfang der erweitert pfändbaren Beträge gelockert. ${ }^{788}$ Mit dem Insolvenzverfahren endet aber auch das Vollstreckungshindernis des § 89 II InsO. Hinsichtlich eines sich anschließenden Restschuldbefreiungsverfahrens stellt § 294 I InsO in Fortführung des Gedankens der fortwirkenden einheitlichen Haftungsverwirklichung aus $\S 89$ InsO nur für Insolvenzgläubiger ein aufrechtzuerhaltendes Vollstreckungsverbot auf, so dass für Neugläubiger während der Treuhandperiode keine Vollstreckungsbeschränkungen bestehen. ${ }^{799}$ Sie können deswegen in das freie, nicht auf den Treuhänder übertragene Vermögen des Schuldners vollstrecken. ${ }^{790}$

Trotz des eindeutigen Wortlauts des $\S 178$ III InsO wird die Frage aufgeworfen, ${ }^{791}$ ob die Rechtskraftwirkung tatsächlich erst der Eintragung der Feststellung in die Tabelle ( $\S 178$ III InsO) und nicht schon der Feststellung der Insolvenzforderung als solches zukommt (§ 178 I 1 InsO), also dem Nichtbestreiten im Prüfungstermin oder der Rücknahme eines Widerspruchs. Insofern lägen hier Unstimmigkeiten innerhalb des § 178 InsO vor.

Gegen die konstitutive Rechtskraftwirkung der Eintragung wird vorgebracht, dass der rechtfertigende Grund für die in § 178 III InsO beschriebene (Feststellungs-)Wirkung auf der vorhergehenden und ausschließlich dem Insolvenzverwalter und der Insolvenzgläubigergemeinschaft obliegenden Entscheidung beruhe, die Ausfluss der Befugnis ist, gegenseitig die haftungsrechtlichen Berechtigungen anzuerkennen, ${ }^{792}$ so dass bereits dem Vorliegen der Feststellungsvoraussetzungen - also durch unterlassenen bzw. beseitigten Widerspruch - konstitutive Rechtskraftwirkung zukommen müsste („...gilt als festgestellt...“, § 178 I

788 BGH ZInsO 2008, 39 (40); OLG Zweibrücken ZInsO 2001, 625 (625f.); Kübler/Prütting/Bork/Lüke, § 89 Rn.29.

789 OLG Zweibrücken ZInsO 2001, 625 (625f.); FK/Ahrens, § 294 Rn.9; Braun/Lang, $\S 294$ Rn.3.

790 Siehe zu weiteren Einzelheiten bei FK/Ahrens, § 294 Rn.9ff.

791 KS/Eckardt, S.761 Rn.36.

792 Bereits unter D.IV.5.a) (S.53ff.). 
1 InsO). Der Eintrag in der Tabelle hätte dann lediglich deklaratorische Bedeutung, so dass der Wortlaut des $\S 178$ III InsO hinter den des $\S 178$ I InsO zurücktrete. ${ }^{793}$ Richtig ist, dass das Insolvenzgericht bei der freiwilligen Insolvenzforderungsfeststellung durch die Verfahrensbeteiligten untereinander im Prüfungstermin - im Gegensatz zum Prozessgericht im Rahmen der erzwungenen Feststellung im ordentlichen Prozessweg (§§ 179ff. InsO) - durch seine gem. § 178 II InsO vorzunehmenden Tabelleneintragungen keine eigene, erkennende Entscheidung über den Bestand der Insolvenzforderung trifft, sondern lediglich eine rein beurkundende Tätigkeit hinsichtlich des Verhaltens und der Entscheidungen der Verfahrensbeteiligten ausübt. ${ }^{794}$ Bezieht man jedoch hinsichtlich der vorgebrachten „Unstimmigkeit“ den Gesetzestext der vormaligen Konkursordnung in die Betrachtung mit ein, so zeigt sich, dass dieser scheinbare Konflikt innerhalb des § 178 InsO unter der Konkursordnung vom Gesetzgeber eindeutiger, nämlich in zwei Paragraphen geregelt war. § 178 I InsO entspricht dem damaligen § 144 I KO und $\S 178$ II, III InsO dem $\S 145$ I, II KO. Dies zeigt, dass der Gesetzgeber bewusst zwischen den Voraussetzungen der Feststellung (§ $144 \mathrm{I}$ KO bzw. § 178 I InsO) einerseits und der Wirkung durch ihre Eintragung in die Tabelle ( $§ 145 \mathrm{KO}$ bzw. $\S 178$ II, III InsO) andererseits unterscheiden wollte. $^{795}$ Der nunmehrigen Zusammenfassung dieser vormaligen Differenzierung in einen Paragraphen innerhalb der Insolvenzordnung trägt die Überschrift „Voraussetzung und Wirkung der Feststellung“ des § 178 InsO Rechnung. Jedenfalls wird deutlich, dass trotz einer hier fehlenden insolvenzgerichtlichen Entscheidung im Prüfungstermin über den Bestand der Insolvenzforderung die Rechtskraftwirkung der Feststellung aufgrund ihrer Tragweite zumindest an einen formellen Akt des Insolvenzgerichts, nämlich an die Eintragung in die Tabelle, gebunden sein soll. ${ }^{796}$ Dies entspricht dem in der Natur der Sache liegenden Prinzip, Rechtskraftwirkungen nur an förmliche Akte des

793 So KS/Eckardt, S.761f. Rn.36f.; Andres/Leithaus/Leithaus, § 178 Rn.3; Carl, Teilnahmerechte, S.75.

794 Bereits unter D.IV.5.a) (S.53ff.).

795 Vgl. auch Jaeger/Weber, KO, § 144 Rn.1.

796 KO-Motive, S.363 = Hahn, KO, S.326: „Der [§ 178 II, III InsO] verordnet die Eintragung des Ergebnisses der Prüfungsverhandlung in die Tabelle und zwar mit Rücksicht auf die Bedeutung dieser Eintragung [Rechtskraftwirkung]...die Eintragung durch das Gericht...“” 
Gerichts zu knüpfen, die im Übrigen zur Feststellung ihres Inhalts und damit des Gegenstandes der rechtskräftigen Feststellung schriftlich fixiert werden müssen. ${ }^{797}$ Es ist eine - wie eingangs bereits dargestellt - durch Zweckmäßigkeitserwägungen gerechtfertigte und auf der eigenartigen Vorschrift des § 178 III InsO beruhende Besonderheit, dass die Eintragung ausdrücklicher oder stillschweigender Anerkenntnisse (§ 178 I InsO), also der (bloße) Vermerk der freiwilligen Feststellung der Insolvenzforderungen durch das Insolvenzgericht (§ 178 II InsO), unmittelbar wie ein rechtskräftiges Urteil wirken soll. ${ }^{798}$ Daher kann der Auffassung, die Rechtskraftwirkung des $\S 178$ III InsO direkt an das Vorliegen der Feststellungsvoraussetzungen des $\S 178$ I InsO - also des Nichtbestreitens - zu knüpfen, nicht gefolgt werden. Vielmehr legt § 178 I InsO die Voraussetzungen der Feststellung fest, § 178 III InsO ihren Vollzug. ${ }^{799}$ Somit kommt erst dem Feststellungsvermerk des Insolvenzgerichts in der Tabelle, wie es auch dem eindeutigen Wortlaut des § 178 III InsO entspricht, konstitutive Wirkung zu. ${ }^{800}$ Dies führt insbesondere zu einer größeren Rechtssicherheit für den Insolvenzverwalter, wenn ein Insolvenzgläubiger behauptet, seine Insolvenzforderung sei trotz Eintragung eines Widerspruchs im Prüfungstermin festgestellt worden. Hier zeigt sich deutlich die Schwäche der Gegenansicht, die dem Feststellungsvermerk lediglich deklaratorische Wirkung zukommen lassen will, so dass selbst die Gegenansicht den Vermerk gleichwohl als „formal maßgeblich“ ansieht. ${ }^{801}$

Spellenberg, Gegenstand, S.6.

BHG ZIP 2006, 1410 (1412); Jaeger/Weber, KO, § 145 Rn.3.

99 MüKo-InsO/Schumacher, § 178 Rn.58; Jaeger/Weber, KO, $§ 145$ Rn.3; Wilmowski/Kurlbaum, KO, § 145 Rn.5; Spellenberg, Gegenstand, S.6.

800 RGZ 22, 153 (155); HK/Irschlinger, § 178 Rn.4; Uhlenbruck/Uhlenbruck, § 178 Rn.5; MüKo-InsO/Schumacher, § 178 Rn.56; HHK/Preß/Henningsmeier, § 178 Rn.19; Graf-Schlicker/Graf-Schlicker, § 178 Rn.15; Häsemeyer, InsO, Rn. 22.18; Mohrbutter/Ringstmeier/Ernestus, $\S 11$ Rn.37; Hess, InsO, $\S 178$ Rn.25; Jaeger/Weber, KO, § 145 Rn.3.

801 KS/Eckardt, S.762 Rn.37. 


\section{Gegenstand der Feststellung}

Im Zusammenhang mit der Rechtskraftwirkung des § 178 III InsO stellt sich die Frage, was genau Gegenstand der Feststellung ist und damit in Rechtskraft erwächst. Diese Frage ist von jeher umstritten. ${ }^{802}$ Derzeit werden zwei Auffassungen vertreten:

Nach einer Auffassung bildet den Gegenstand des insolvenzrechtlichen Feststellungsverfahrens das insolvenzspezifische Haftungsrecht des Insolvenzgläubigers an der Insolvenzmasse, also seinen subjektiven Anteil an der gemeinschaftlichen Haftungszuweisung. ${ }^{803}$ Nach der Gegenauffassung und herrschenden Meinung ist nicht lediglich das insolvenzspezifische Haftungsrecht, sondern die Forderung in ihrer Eigenschaft als Insolvenzforderung bestimmten Ranges und bestimmten Betrages Gegenstand der Feststellung. ${ }^{804}$ Bedeutung hat diese Streitfrage für die Präjudizwirkung der Feststellung. Sieht man die Forderung gegen den Schuldner als Gegenstand der Feststellung an, so kann z.B. der Insolvenzverwalter in einem späteren Absonderungsrechtsstreit mit dem Insolvenzgläubiger den Bestand der zuvor festgestellten (Insolvenz-) Forderung nicht mehr wirksam bestreiten. Wird nur der geltend gemachte Rang bestritten und hat das Insolvenzgericht zur Anmeldung von Insolvenzforderungen des nicht bestrittenen Nachrangs aufgefordert, so ist das Gericht im Prozess um den besseren Rang ebenfalls an die vorangegangene Feststellung der Insolvenzforderung gebunden. ${ }^{805}$ Das gilt ebenso, wenn der Insolvenzgläubiger die Forderung zunächst als nachrangige Insolvenzforderung anmeldet und feststellen lässt und erst später einen besseren Rang anmeldet. ${ }^{806}$ Nach der Auffassung vom Haftungsrecht als Gegenstand der Feststellung ist in diesen Fällen das

\footnotetext{
802 Zum älteren Meinungsstand siehe bei Spellenberg, Gegenstand, S.25ff., 81ff.

803 KS-Eckardt, S.744 Rn.1f., S.763 Rn.38ff.; Jaeger/Henckel, KO, § 29 Rn.19; Henckel, in: FS/Michaelis, S.167; Spellenberg, Gegenstand, S.81ff.; Jaeger/Henckel, InsO, $\S 38$ Rn.4; MüKo-InsO/Schumacher, § 178 Rn.15; HHK/Herchen, § 179 Rn.17.

804 RGZ 55, 157 (160); BGH KTS 1983, 602 (605f.); BGH WM 1961, 427 (427f.,429); OLG Schleswig ZInsO 2004, 687 (687); Graf-Schlicker/Graf-Schlicker, § 178 Rn.5; Kübler/Prütting/Bork/Pape, § 179 Rn.11; Hess, InsO, § 178 Rn.29; Smid/Smid, § 178 Rn.11; Uhlenbruck/Uhlenbruck, § 178 Rn.12f.; Häsemeyer, InsO, Rn.22.03, 22.20; Jaeger/Weber, KO, § 146 Rn.13

805 RGZ 144, 246 (248f.); BAG KTS 1967, 231 (233); Jaeger/Weber, KO, § 145 Rn.5; Smid/Smid, § 178 Rn.11; Uhlenbruck/Uhlenbruck, § 178 Rn.7 m.w.N.

806 Vgl. RGZ 149, 257 (267ff.) zur nachträglichen Anmeldung eines Vorrechts nach der Konkursordnung.
} 
Gericht im nachfolgenden Feststellungsprozess hingegen nicht gehindert, das Bestehen der Insolvenzforderung abzulehnen. Ob die Forderung besteht, für die das Haftungsrecht beansprucht wird, ob sie den beanspruchten Rang hat und ob sie als Insolvenzforderung zu qualifizieren ist, sind nach dieser Ansicht nur Vorfragen, über die nicht rechtkräftig entschieden wird. ${ }^{807}$

Die Ansicht vom Haftungsrecht macht für sich geltend, dass der Insolvenzgläubiger nur für die Feststellung seines Anteils an der Masse ein schutzwürdiges Interesse habe und für eine (Zwischen-)Feststellung einzelner Voraussetzungen des Haftungsrechts im Feststellungsverfahren kein Raum sei. ${ }^{808}$ Denn die Rechtskraftfiktion des $§ 178$ III InsO solle allein der Absicherung des Prüfungsergebnisses gegenüber nachträglichen Widersprüchen dienen, womit die Vorstellung unvereinbar wäre, dass die Insolvenzforderung gegen den Schuldner in jeglicher Hinsicht, in der sie im Verhältnis zum Insolvenzverwalter oder einem anderen Insolvenzgläubiger innerhalb oder außerhalb des Verfahrens von Relevanz sein könnte, rechtskraftfähig festgestellt würde. ${ }^{809}$ Hierfür wird vorgebracht, dass auch die Materialien der Konkursordnung zu § 164 II KO (= § 201 II InsO) hinsichtlich der Rechtskraftwirkung des Feststellungsvermerks über das Verfahren hinaus davon sprechen, dass „die Anmeldung und Feststellung der Konkursforderungen im Konkursverfahren und in den Sonderprozessen auf den Kreis des Konkursrechts beschränkt“ sei; mit ihnen werde nur „das Recht der Teilnahme an den Beschlussfassungen über die Konkursteilnahme und an der Verteilung derselben“ verfolgt. ${ }^{810}$ Nicht erwähnt wird jedoch, dass an gleicher Stelle auch gesagt wird und was gegen das Konstrukt des Haftungsrechts spricht, dass das „Theilnahmerecht auf dem Forderungsrecht des Gläubigers gegen den Schuldner beruht, und...die Prüfung den vollen Umfang der Forderung umfaßt“. Auch wird ausdrücklich darauf hingewiesen, dass die Gesetzgebung indes nicht „auf

807 KS/Eckardt, S.765 Rn.42; MüKo-InsO/Schumacher, § 178 Rn.14.

808 Henckel, in: FS/Michaelis, S.152f., 167; KS/Eckardt, S.765 Rn.41; MüKoInsO/Schumacher, § 178 Rn.15.

809 KS/Eckardt, S.764 Rn.40. Gleichzeitig wird aber darauf verwiesen, dass § 178 III InsO nur diejenigen Rechtsbehelfe und Einwendungen gegen die Feststellung zulässt, die gegen ein rechtskräftiges Urteil (§ 322 I ZPO) gegeben wären. KS/Eckardt, S.764 Rn.38, 45.

810 KO-Motive, S.384 = Hahn, KO, S.343; KS/Eckardt, S.764 Rn.40. 
dem bloßen Standpunkt juristischer Konsequenz" stehen bleiben dürfe, denn: „dasselbe Streitverhältnis liegt vor damals bei der Prüfung, wie jetzt bei der Nachforderung; die Feststellung ist ergangen“. „Es würde unnöthigen und kostspieligen Aufschub verursachen, bloßen Ausflüchten Thür und Thor öffnen, im Interesse des Schuldners nicht unbedingt geboten sein, das Interesse der Gläubiger aber unzweifelhaft gefährden, wenn dieselben genöthigt werden sollten, trotz der ihre Forderungen mittelbar anerkennenden Tabelle und Urtheile sie gegen den Schuldner von neuem durch Klage zu verfolgen.“"811 Die Formulierung der (lediglich) „mittelbaren“ Anerkennung wird hier im Kontext der Materialien zu § 164 II KO (= 201 II InsO) und in Bezug auf den Schuldner wohl deshalb gewählt worden sein und daher nicht überbewertet werden dürfen, weil der Schuldner selbst im Verfahren keine Forderung unmittelbar feststellend anerkennen oder ihrer Feststellung widersprechen kann. ${ }^{812}$ Über die Materialien und Motive zur Konkursordnung hinaus sprach sich der Gesetzeswortlaut der Konkursordnung über den Gegenstand der Feststellung selbst wie folgt aus: der Anmeldende hat die Feststellung der „streitig gebliebenen Forderung“ zu betreiben ( $§ 146$ I 1 KO), der Bestreitende widerspricht der „Forderung“ ( $§ 146$ VI KO), das der Feststellungsklage stattgebende Urteil stellt „die Forderung“ fest (§ 147 S.1 KO), die „Forderung“ ist infolge eines der Prüfung erhobenen Widerspruchs „im Prozesse befangen“ (§ 168 Nr.1 KO). Der Inhalt der angeführten Paragraphen der Konkursordnung wurde dabei sinngemäß vom Gesetzgeber in die Insolvenzordnung übernommen. ${ }^{813}$ Den Aufschluss über den Feststellungsgegenstand gibt der Gesetzgeber in seiner Begründung zum Änderungsgesetz der Insolvenzordnung nunmehr jedoch auch selbst, ${ }^{814}$ wenn es zum Forderungsattribut der vorsätzlich begangenen unerlaubten Handlung heißt, verfahrenstechnisch lasse sich ein solches „Privileg“ des Insolvenzgläubigers, wie es in den neuen §§ 174 II, 302 Nr.1 InsO geregelt sei, „wie die Behandlung eines Konkursvorrechts nach

811 KO-Motive, S.384 = Hahn, KO, S.343f.

812 Außer in der inzwischen eingeführten Verfahrensbesonderheit der Eigenverwaltung des Schuldners. Siehe bereits unter E.I.4.d)aa) (S.157ff.).

813 Siehe Begr. zu $\S \S$ 208-211 ( $\S \S ~ 179-183$ InsO) und $\S 217$ ( $\S 189$ InsO) RegE, BT-Drucks. 12/2443, S.185f.

814 Begr. zu Nr.12 (§ 174 II InsO) RegE, BT-Drucks. 14/5680, S.24f. 
altem Recht behandeln“. Nach den Vorstellungen des Gesetzgebers ${ }^{815}$ sollen wie im früheren isolierten Vorrechtsstreit nach der Konkursordnung auch bei dem Streit, ob der Insolvenzforderung eine vorsätzlich begangene unerlaubte Handlung des Schuldners zugrunde liegt, alle gegen den Bestand gerichteten Einwendungen durch die Rechtskraftwirkung des Feststellungsvermerks abgeschnitten sein. Widerspreche der Schuldner nicht, so werde der Vortrag des Insolvenzgläubigers, die Forderung stamme aus einer vorsätzlich begangenen unerlaubten Handlung, in die Tabelle eingetragen, was nach § 178 III InsO wie ein rechtskräftiges Urteil gegenüber dem Insolvenzverwalter und den Insolvenzgläubigern wirke. Fehle die Feststellung hinsichtlich einer vorsätzlich begangenen unerlaubten Handlung in der Tabelle, könne sich der Insolvenzgläubiger auf einen angeblichen Ausschluss seiner Insolvenzforderung von der Restschuldbefreiung nicht mehr berufen. ${ }^{816}$ Mit diesen Ausführungen folgt der Gesetzgeber somit eindeutig der Auffassung der bisher herrschenden Meinung, so dass Gegenstand der Feststellung nur die Forderung in ihrer Eigenschaft als Insolvenzforderung bestimmten Ranges und bestimmten Betrages sein kann. Wünschenswert und konsequent wäre es daher gewesen, wenn der Gesetzgeber dies auch im Wortlaut des § 178 III InsO klargestellt hätte, indem die Eintragung in die Tabelle für die festgestellte Insolvenzforderung nicht nur ihrem Betrag und Rang nach Rechtskraftwirkung zukommt, sondern auch dem Grunde nach. ${ }^{817}$

Fraglich ist, ob mit der „Forderung“ das materielle Recht oder ein eigenständiger „prozessualer“ Anspruch gemeint ist. Die Insolvenzforderung bestimmt sich ohne Rücksicht auf die materiellrechtlichen Rechtsbehauptungen des Anmeldenden nach dem in der Anmeldung anzugebenden Betrag und dem zur Begründung vorgetragenen Lebenssachverhalt (dem „Grund der Forderung“ im Sinne des § 174 II InsO). Die rechtlichen Aspekte, nach denen der Anspruch zu würdigen ist, sind in der Anmeldung nicht anzugeben. ${ }^{818}$ Daher liegt hier

\footnotetext{
Begr. zu Nr.12 (§ 174 II InsO) RegE, BT-Drucks. 14/5680, S.24f.

Siehe bereits unter D.IV.3.a) (S.44ff.) und E.III.5.c) (S.225ff.).

So auch Uhlenbruck/Uhlenbruck, § 178 Rn.13.

818 RGZ 93, 14 (14); Uhlenbruck/Uhlenbruck, § 178 Rn.16; eine Ausnahme bildet lediglich die vom Anmeldenden geforderten Angaben hinsichtlich des zusätzlichen Schuldgrundes der vorsätzlich begangenen unerlaubten Handlung und dessen Bedeutung für den Schuldner. Siehe bereits unter D.IV.3.a) (S.44ff.) und E.I.4.f)bb)(2) (S.175ff.).
} 
der prozessuale Anspruch näher. Wird zudem die Forderung bestritten, so ist die Feststellung gegebenenfalls durch Aufnahme eines „über die Forderung“ anhängigen Rechtsstreits zu betreiben (§§ 180 II, 184 II InsO). Gegenstand des anhängigen Rechtsstreits ist aber ebenfalls nicht das materielle Recht, sondern der prozessuale Anspruch. Wenn daher in Anlehnung an den Sprachgebrauch der Konkurs- und der Insolvenzordnung von der „Forderung“ die Rede ist, so ist damit inhaltlich ein von den materiellen Rechtsbehauptungen losgelöster, durch den angemeldeten Betrag und den dazu vorgetragenen Lebenssachverhalt bestimmter „prozessualer“ Anspruch gemeint. ${ }^{819}$

Damit ergibt sich mit der Forderung - als prozessualer Anspruch - auch ein durchgehend einheitlicher Verfahrensgegenstand, ${ }^{820}$ sowohl im freiwilligen wie streitigen Feststellungsverfahren, sei es in einer neu zu erhebenden Klage ( $\S 180$ I InsO) oder sei es in dem durch Wiederaufnahme ( $\S 180$ II InsO) eines durch die Eröffnung des Insolvenzverfahrens unterbrochenen Rechtsstreits (§ 240 ZPO). ${ }^{821}$ Nichts anderes liegt auch im Interesse des anmeldenden Insolvenzgläubigers, als „seine Forderung“ durch ihre Feststellung im Insolvenzverfahren - zumindest teilweise - befriedigt zu sehen und darüber hinaus, eben durch Feststellung der Forderung, nach Verfahrensbeendigung einen Vollstreckungstitel zu erlangen (§ $201 \mathrm{II}$ InsO). ${ }^{822}$

819 MüKo-InsO/Schumacher, § 178 Rn.12. Zur prozessualen Rechtskrafttheorie siehe auch Ausführungen bei MüKo-InsO/Bitter, § 45 Rn.42.

820 Zu (un-)nötigen Differenzierungen der Rechtskraftwirkungen hinsichtlich § 178 III und § 201 II InsO bei der Annahme des Haftungsrechts als Feststellungsgegenstandes - die nach angeführten und eindeutigen Aussagen vom Gesetzgeber keineswegs gewollt sein können - siehe MüKo-InsO/Schumacher, § 178 Rn.15f.; KS/Eckardt, S.764 Rn.39 mit Fn.81.

821 Vgl. auch BGH ZInsO 2005, 372 (372f.).

822 Das Interesse des Insolvenzgläubigers, auch einen Vollstreckungstitel gegen den Schuldner erwirken zu können, scheint die Ansicht vom Haftungsrecht in ihrer Betrachtung völlig auszublenden. Siehe MüKo-InsO/Schumacher, § 178 Rn.15. 


\section{Feststellung von Nichtinsolvenzforderungen}

Feststellungsgegenstand ist somit die Forderung gegen den Schuldner in ihrer durch §§ 38, 39 InsO festgelegten Eigenschaft als Insolvenzforderung mit einem bestimmten Betrag und einem bestimmten Rang (§ 178 III InsO). Daher sind Forderungen, die nicht der materiellrechtlichen Definition des $\S 38$ InsO entsprechen, ${ }^{823}$ z.B. Masseansprüche die den $\S \S 53 f f$. InsO unterfallen, auch nicht zur Tabelle feststellbar. Werden diese Forderungen dennoch, wenn auch irrtümlich, zur Tabelle angemeldet, aufgenommen und eventuell sogar „festgestellt“, so entfaltet dies keine Rechtskraftwirkung, weil die Forderung dadurch nicht $\mathrm{zu}$ einer Insolvenzforderung gem. $\S \S 38,39$ InsO wird. ${ }^{824}$ Der Feststellungsvermerk ist dann gegenstandslos und von Amts wegen zu berichtigen. ${ }^{825}$ Der Grund hierfür liegt folgerichtig darin, dass die rechtliche Einordnung einer Forderung als Insolvenzforderung im Sinne der §§ 38, 39 InsO nicht zur Disposition der Verfahrensbeteiligten steht, ${ }^{826}$ auch wenn die Prüfung und Feststellung aller angemeldeten Forderungen allein den widerspruchsberechtigten Verfahrensteilnehmern obliegt. $^{827}$ Wenn der Wortlaut und die Systematik der $\S \S 174 f f$. InsO zwingend vorschreibt, dass nur bestimmte Forderungen ( $\S 38$ InsO) im Anmelde- und Feststellungsverfahren geltend gemacht werden können, dann können der Insolvenzverwalter und die übrigen widerspruchsberechtigten Insolvenzgläubiger hinsichtlich anderer Forderungen, die diesen Vorgaben nicht entsprechen (z.B. Masseforderungen gem. §§ 53ff. InsO), kein wirksames Anerkenntnis zu Lasten der Masse abgeben. Ihre Dispositionsbefugnis kann sich nur so weit erstrecken, wie es ihnen die Insolvenzordnung vorgibt. Dies hat im Umkehrschluss natürlich zur Folge, dass die „Feststellung“ zur Tabelle keine Sperrwirkung für die

\footnotetext{
823 Siehe bereits unter C.I. (S.10ff.).

824 Herrschende Meinung BGH ZIP 2006, 1410 (1411f); ZIP 1991, 456 (456); BAG ZIP 1989, 1205 (1205); OLG Schlesien, ZInsO 2004, 687 (687); Balz, EWiR 1989, 745 (746); HK/Irschlinger, § 178 Rn.6; Kübler/Prütting/Bork/Pape, § 178 Rn.10f.; Uhlenbruck/Uhlenbruck, § 178 Rn.17; MüKo-InsO/Schumacher, § 178 Rn.65 m.w.N. HK/Irschlinger, § 178 Rn.6; Gottwald/Eickmann, § 64 Rn.31; Jaeger/Henckel, KO, $\S 25$ Rn.45; Hess, InsO, § 178 Rn.37, 41.

826 BGH ZIP 2006, $1410 \quad$ (1411); BAG ZIP 1989, $1205 \quad$ (1206); Mohrbutter/Ringstmeier/Ernestus, § 11 Rn.18; Kübler/Prütting/Bork/Pape, § 174 Rn.18 jeweils m.w.N.

827 Abzulehnen daher KS/Eckardt, S.768 Rn.46; MüKo-InsO/Schumacher, § 178 Rn.66, die den Verfahrensbeteiligten eine solche Disposition zusprechen wollen.
} 
Geltendmachung solcher Ansprüche entfaltet, die keine Insolvenzforderungen sind. ${ }^{828}$

Anders wiederum liegt der Fall, wenn eine nachrangige Insolvenzforderung ( $§ 39$ InsO), ohne Aufforderung zu ihrer Anmeldung durch das Insolvenzgericht ( $§ 174$ III 1 InsO), als nicht nachrangige Insolvenzforderung angemeldet und festgestellt wird. Wegen ihrer generellen Einordnung als Insolvenzforderung (§§ 38, 39 InsO) ${ }^{829}$ unterliegen nämlich grundsätzlich auch nachrangige Insolvenzforderungen der Dispositionsbefugnis der Widerspruchsberechtigten. Sollte also eine unzulässig weil ohne Aufforderung gem. § 178 III 1 InsO angemeldete nachrangige Forderung als Insolvenzforderung - durch die in diesem Fall Dispositionsbefugten - zur Tabelle festgestellt werden, erwächst dieser Feststellungsvermerk in Rechtskraft. ${ }^{830}$

\section{Protokollierung des Prüfungsergebnisses}

$\S 178$ II InsO regelt, was das Insolvenzgericht als Ergebnisse des Prüfungstermins $\mathrm{zu}$ beurkunden hat. Neben der allgemeinen Protokollierung des Prüfungstermins (§ 4 InsO i.V.m. §§ 159ff. ZPO) ${ }^{831}$ hat das Insolvenzgericht für jede angemeldete Forderung in die Tabelle einzutragen, inwieweit die Forderung ihrem Grund, Betrag und ihrem Rang nach festgestellt ist oder wer der Feststellung widersprochen hat. Auch ein Widerspruch des Schuldners ist gem. § 178 II 2 InsO in die Tabelle aufzunehmen. Dies ist nötig, da ein nicht beseitigter Widerspruch des Schuldners zwar nicht die Feststellung der angemeldeten Forderung, nach § 201 II InsO aber die Titulierungswirkung des Tabelleneintrags verhindert und damit eine nachinsolvenzliche Zwangsvollstreckung aus dem Tabelleneintrag gegen ihn persönlich ausschließt. Bei einem

828 Kübler/Prütting/Bork/Pape, § 174 Rn.11; Uhlenbruck/Uhlenbruck, § 178 Rn.17; Hess, InsO, § 178 Rn.37f. m.w.N. Allein in der Anmeldung einer Forderung als Insolvenzforderung liegt kein Verzicht auf die Masseschuld. - RGZ 98, 136 (137); BGH ZIP 2006, 1410 (1412).

829 Siehe bereits unter D.IV.5. (S.53ff.).

830 KS/Eckardt, S.768 Rn.46; wohl auch Kübler/Prütting/Bork/Pape, § 178 Rn.11; vgl auch BGHZ 113, 381 (383) zur Feststellung einer Forderung aus kapitalersetzenden Darlehen unter der Konkursordnung.

831 „Daß zugleich über die Verhandlung ein Protokoll aufzunehmen ist, ist selbstverständlich.“ KO-Motive, S.363 = Hahn, KO, S.326 zu den §§ 132, 133 Konkursordnung; siehe auch MüKo-Inso/Schumacher, § 178 Rn.49 m.w.N. 
(teilweisen/isolierten) Widerspruch ist weiterhin die genaue Widerspruchsrichtung zu vermerken (z.B. gegen die Höhe des Betrags oder gegen die Qualifizierung der angemeldeten Insolvenzforderung als eine solche aus vorsätzlich begangener unerlaubter Handlung des Schuldners). ${ }^{832}$ Wobei sich die Tragweite des Widerspruchs aus dem Vermerk über die Feststellung und umgekehrt ergibt. ${ }^{83} \mathrm{Zu}$ einer Feststellung des Ranges der Insolvenzforderung kann es nur noch kommen, wenn das Insolvenzgericht zur Anmeldung nachrangiger Insolvenzforderungen aufgefordert hat. Persönliche Forderungen von Insolvenzgläubigern, die durch ein Absonderungsrecht (§§ 49-52 InsO) dinglich abgesichert sind (so genannte Ausfallforderungen), sind uneingeschränkt mit ihrem angemeldeten Betrag zur Insolvenztabelle festzustellen, ${ }^{834}$ da auch sie vollwertige Insolvenzforderungen sind. ${ }^{835}$ Der Charakter als Ausfallforderung wird erst im Verteilungsverfahren relevant, sollte aber gleichwohl zur allgemeinen Information für alle Verfahrensbeteiligten - insbesondere dem Insolvenzverwalter als Gedächtnisstütze - in der Tabelle vermerkt werden. Erst im Verteilungsverfahren ist es dann Sache des Insolvenzgläubigers, seinen Ausfall nachzuweisen, um bei der Verteilung berücksichtigt zu werden (vgl. § 190 InsO).

\section{a) Vorabeintragung des Prüfungsergebnisses und Unterschrift(en)}

Die Eintragung der Ergebnisse hat aufgrund der Bedeutung und Tragweite der Tabellenvermerke gem. § 178 II 1 InsO durch das Insolvenzgericht zu erfolgen, also in der Regel durch den Rechtspfleger (§ 18 RPflG) und/oder gegebenenfalls auch den die Tabelle führenden und den Prüfungstermin protokollierenden Urkundsbeamten der Geschäftsstelle (vgl. § 159 I 2 ZPO) ${ }^{836}$ Vielfach trägt jedoch bereits der Insolvenzverwalter das $\mathrm{zu}$

\footnotetext{
832 Siehe bereits unter E.I.4.c)bb) (S.145ff.). Siehe zu Formulierungsbeispiele etwa bei Uhlenbruck/Uhlenbruck, § 178 Rn.3.

833 Nerlich/Römermann/Becker, § 178 Rn.16.

834 RGZ 139, 83 (86f.); BGH WM 1957, 1225 (1226); BGH WM 1961, 427 (429); Kübler/Prütting/Bork/Pape, § 178 Rn.12; Uhlenbruck/Uhlenbruck, § 178 Rn.18; Klasmeyer/Elsner, in: FS/Merz, S.306; Mandlik, RPfleger 1980, 143 (143f.).

835 Siehe bereits unter C.II. (S.16ff.) und D.IV.3.c) (S.48f.).

836 Siehe bereits unter E.I.4.c)bb) (S.145ff.).
} 
erwartende Prüfungsergebnis vorbereitend in die von ihm mittels EDV erstellten Tabelle ( $§ 5$ IV InsO) ein. Durch dessen Tabellenführungsbefugnis bis zur Niederlegung der Tabelle beim Insolvenzgericht wird hiergegen nichts einzuwenden sein, sondern ist dem zügigen Fortgang des Prüfungstermins nur zuträglich und hilft Schreib- und Hörfehler im Prüfungstermin vermeiden. Weil nach der Niederlegung der Tabelle die Tabellenführung auf das Insolvenzgericht übergeht, ${ }^{837}$ haben die eventuellen Berichtigungen der Voreintragungen und die übrigen Tabelleneinträge - sei es per EDV oder handschriftlich - jedenfalls gem. $\S 178$ II 1 InsO vom Insolvenzgericht selbst zu erfolgen. ${ }^{838}$ Zudem sind sämtliche angemeldeten Insolvenzforderungen pauschal zu prüfen/aufzurufen und die eventuell voreingetragenen Widersprüche für ihre Wirksamkeit im Prüfungstermin erneut zu erheben/bekräftigen und einzeln zu erörtern, ${ }^{839}$ so dass sich das Insolvenzgericht von der Richtigkeit der Vorabeintragungen durch den Insolvenzverwalter überzeugen und das Ergebnis der Prüfung durch abschließende Unterschrift(en) beurkunden kann. Lässt man diese Vorabeintragungen durch den Insolvenzverwalter daher zu, so muss dies jedoch zu Folge haben, dass die nicht einheitlich beantwortete Frage, ob jeder Tabelleneintrag einzeln vom Rechtspfleger (und eventuell vom Urkundsbeamten) zu unterschreiben ist ${ }^{840}$ oder ob eine „pauschale“ Unterschrift unter das Sitzungsprotokoll bzw. die Sammelliste ausreicht, ${ }^{841}$ dahingehend zu beantworten sein wird, dass jeder Vermerk einzeln zu unterzeichnen ist. Nur so wird generell sichergestellt und auch $\S 178$ II 1 InsO ausreichend Rechnung getragen, dass sich das Insolvenzgericht mit jeder einzelnen Eintragung des Prüfungsergebnisses - sei sie nun selbst oder vorab vom Insolvenzverwalter vorgenommen worden - auseinandergesetzt und bestätigt hat.

37 Siehe bereits unter D.V.3. (S.87ff.).

838 Teilweise einzuschränken sind daher die Ansichten von Kübler/Prütting/Bork/Pape, $\S 178$ Rn.3; MüKo-InsO/Schumacher, § 178 Rn.49; KS/Eckardt, S.761 Rn.35.

839 Siehe auch unter E.I.3. (S.137ff.).

840 Graf-Schlicker/Graf-Schlicker, § 178 Rn.9; Nerlich/Römermann/Becker, § 178 Rn.14; FK/Kießner, § 178 Rn.12; Kübler/Prütting/Bork/Pape, § 178 Rn.5; Hess, InsO, § 178 Rn.2; Kuhn/Uhlenbruck, KO, § 145 Rn.1; Jaeger/Weber, KO, § 145 Rn.1; vgl. auch Muster 17 zu § 15 IV Aktenordnung.

841 So Uhlenbruck/Uhlenbruck, § 178 Rn.2; Haarmeyer/Wutzke/Förster, Kap.7 Rn.56; Kübler, in: FS/Henckel, S.508; Gottwald/Eickmann, § 64 Rn.18; MüKoInsO/Schumacher, § 178 Rn.50. 


\section{b) Feststellungsvermerk auf Urkunden}

Auf Wechseln, Titeln und sonstigen Schuldurkunden ist vom Urkundsbeamten der Geschäftsstelle gem. § 178 II 3 InsO mit Unterschrift und Amtssiegel zu vermerken, inwieweit die Feststellung der Insolvenzforderung erfolgt ist. Der Vermerk hat dabei keine konstitutive Wirkung, sondern verlautbart nur das Ergebnis der Prüfung durch den Inhalt des Feststellungsvermerks. ${ }^{842}$ Durch den am Beispiel des Wechsels erläuterte Begriff der Schuldurkunde in § 178 II 3 InsO ist dieser Wortlaut enger als der in § 174 I 2 InsO gewählte Ausdruck „Urkunden, aus denen sich die Forderung ergibt“, so dass als Schuldurkunde nur zur Forderungsbegründung oder zur Schaffung eines Beweismittels unterzeichnete Schriftstücke in Betracht kommen werden. ${ }^{843}$ Im Gegensatz zur Anmeldung (vgl. § 174 I 2 InsO) kann der Insolvenzgläubiger im Rahmen seiner Vorlage für den Vermerk gem. § 178 II 3 InsO auch keine schlichte Kopie, sondern nur das Original verwenden. Wie bereits ausgeführt, ${ }^{844}$ dient dieser Feststellungsvermerk dem Insolvenzgläubiger in erster Linie dafür, die Verfügung über die Urkunde zu erleichtern. Darüber hinaus verhindert der Vermerk, dass der Insolvenzgläubiger neben dem vollstreckbaren Tabellenauszug des $\S 201$ II InsO über weitere Vollstreckungstitel verfügt, aus denen er die Vollstreckung gegen den Schuldner aufgrund der gleichen Forderung betreiben kann. Für den Fall, dass der Originaltitel bei der Feststellung im Prüfungstermin nicht vorgelegen hat und deshalb ein Feststellungsvermerk nicht vollzogen werden konnte, kann eine Doppeltitulierung dadurch vermieden werden, dass das Insolvenzgericht die spätere Erteilung des vollstreckbaren Tabellenauszugs von der Vorlage der Originalurkunde abhängig macht. Daher ist es ausreichend, wenn der Insolvenzgläubiger dem Urkundsbeamten das Original nach dem Prüfungstermin vorlegt und dieser sich von der Übereinstimmung des Originals mit der bei der Tabelle befindlichen Kopie überzeugt. Nachdem der Feststellungsvermerk vom

\footnotetext{
842 MüKo-InsO/Schumacher, § 178 Rn.53; Uhlenbruck/Uhlenbruck, § 178 Rn.4; HHK/Heß/Henningsmeier, § 178 Rn.17.

843 Nerlich/Römermann/Becker, § 178 Rn.19;

844 Siehe bereits unter D.IV.4.b) (S.50ff.).
} 
Urkundsbeamten gesetzt worden ist, ist die Urkunde dem Insolvenzgläubiger wieder auszuhändigen.

In diesem Zusammenhang stellt sich die Frage, in welchem Verhältnis der ursprüngliche Titel zum jetzigen Feststellungsvermerk der Tabelle bzw. dem vollstreckbaren Tabellenauszug gem. § 201 II InsO steht, der einem rechtkräftigen Urteil gleichsteht.

\section{c) Ursprünglicher Titel - vollstreckbarer Tabellenauszug}

Nach Aufhebung des Insolvenzverfahrens kann jeder Insolvenzgläubiger gem. § 201 II InsO, soweit seine Forderung zur Tabelle festgestellt worden ist (§§ 178 I, III, 201 II 1 InsO), auf Antrag einen vollstreckbaren Tabellenauszug erhalten, mit dem er die Zwangsvollstreckung in das Vermögen des Schuldners betreiben kann, sofern nicht die Vorschriften der Restschuldbefreiung entgegenstehen (§ 201 III InsO). ${ }^{845}$ Hierzu muss der Tabellenvermerk wie bei einem Urteil in dem dafür vorgesehenen Verfahren mit einer Vollstreckungsklausel (§§ 725, 750 ZPO i.V.m. § 4 InsO) versehen werden, weil die Insolvenzordnung keine Sonderregelungen zu den $\S \S 724 \mathrm{ff}$. ZPO enthält und der Tabellenauszug somit nicht automatisch vollstreckbar ist. ${ }^{846}$ Zwar ist in $\S 201$ InsO der Hinweis auf die entsprechende Anwendung der $\S \S 724 f f$. ZPO - wie es noch in $\S 164$ II KO der Fall war - entfallen, jedoch sollte § 201 InsO den § 164 KO sinngemäß übernehmen. Aus dem fehlenden Verweis in § 201 InsO kann nicht geschlossen werden, dass der Gesetzgeber die Erteilung der Vollstreckungsklausel auf den Tabellenauszug für entbehrlich hält. Hierfür spricht zudem die Regelung über streitige Vollstreckungsklauseln im folgenden § 202 I Nr.1 InsO. Entbehrlich ist durch § 178 III InsO lediglich die Feststellung der Rechtskraft. Der Tabellenauszug steht damit entgegen dem Wortlaut des § 178 III InsO nicht einem vollstreckbaren, sondern

845 Vgl. § 294 InsO.

846 Begr. zu $\S \S$ 229, $230 \quad$ (§ 201 InsO) RegE, BT-Drucks. 12/2443, S.187; HK/Irschlinger, § 201 Rn.7; Nerlich/Römermann/Westphal, §§ 201, 202 Rn.19; MüKo-InsO/Hintzen, § 201 Rn.27. 
- wie es auch die §§ 144, 145, 164 II KO zum Ausdruck brachten ${ }^{847}$ einem rechtkräftigen Urteil gleich. ${ }^{848}$

Sinn des Insolvenzverfahrens ist es, die Befriedigung der Insolvenzgläubiger durch das Anmeldungs- und Feststellungsverfahren und dessen Rechtskraftwirkung auf eine neue, unstreitige und gegenüber allen Verfahrensbeteiligten geltende Grundlage zu stellen. ${ }^{849}$ Dies kann für bereits vor Eröffnung des Insolvenzverfahrens vorliegende Vollstreckungstitel nur bedeuten, dass sie, aufgrund ihrer Einbindung in das Verfahren durch Anmeldung mit eventuell insolvenzspezifisch abgeändertem Inhalt ( $\S \S 41 \mathrm{ff}$. InsO) durch ihre Feststellung neu tituliert werden und der ursprüngliche Titel insoweit in seiner Vollstreckungskraft „verdrängt“ wird und für die Nachhaftung nur noch der vollstreckbare Tabellenauszug maßgeblich ist. ${ }^{850}$ Die älteren Auffassungen, dass ein einmal bestehender Titel von einem rechtskräftigen Tabellenauszug gar nicht berührt werde, ${ }^{851}$ oder dass sich der Gläubiger wahlweise des älteren Titels oder des vollstreckbaren Tabellenauszugs bedienen, ${ }^{852}$ oder der Insolvenzgläubiger aus beiden Titeln vollstrecken könne, ${ }^{853}$ können aufgrund der Rechtskraftwirkung des § 201 II InsO nicht in die Insolvenzordnung übertragen werden. ${ }^{854}$ Wäre die erstgenannte Auffassung zutreffend, wäre eine Vollstreckung derselben Forderung nach § 201 II InsO nicht möglich, nach zweiter Ansicht wäre bei Wahl des früheren Titels § 201 II InsO funktionslos, und die letzte Auffassung beruht auf $\S 85$ VglO, wonach aus dem bestätigten Vergleich in Verbindung mit dem berichtigten Gläubigerverzeichnis gegen den Schuldner vollstreckt werden

847 Siehe nur Jaeger/Weber, KO, § 164 Rn.3f.

848 Holzer, NZI 1999, 44 (46); MüKo-InsO/Hintzen, $\S 201$ Rn.21, 27; Uhlenbruck/Uhlenbruck, § 201 Rn.6; Kübler/Prütting/Bork/Holzer, § 201 Rn.9 m.w.N.

849 Siehe bereits unter E.III.2. (S.208ff.).

850 Im Ergebnis ganz herrschende Meinung: RGZ 112, 297 (300f.); 132, 113 (115); BGH ZInsO 2006, 704 (704); Jaeger/Weber, KO, § 164 Rn.6; Kübler/Prütting/Bork/Holzer, § 201 Rn.17; MüKo-InsO/Hintzen, § 201 Rn.37; Uhlenbruck/Uhlenbruck, § 201 Rn.9 m.w.N.

851 So zunächst noch der Gesetzgeber zur Konkursordnung von 1877 in KO-Motive, S.386 = Hahn, KO, S.345.

852 So zu § 164 KO. Siehe nur Gaul, in: FS/Weber, S.155 (156) m.w.N.

853 Nach § 85 VglO konnte aus dem bestätigten Vergleich in Verbindung mit dem berichtigten Gläubigerverzeichnis gegen den Schuldner vollstreckt werden. Der neue Titel erwuchs jedoch nicht in Rechtskraft, so dass er einen früheren Titel weder ersetzte noch verdrängte. RGZ 132, 113 (115); Bley/Mohrbutter, VglO, § 85 Rn.30 m.w.N.

854 Vgl. Küber/Prütting/Holzer, § 201 Rn.14ff.; Uhlenbruck/Uhlenbruck, § 201 Rn.9; ausführlich auch MüKo-InsO/Bitter, § 45 Rn.41, 42-44. 
konnte. Der neue Titel erwuchs nämlich nicht in Rechtskraft, wie es jedoch nunmehr gem. §§ 178 I, III, 201 II 1 InsO der Fall ist, so dass er einen früheren Titel weder ersetzen noch verdrängen konnte.

Die Auffassung, dass der frühere Vollstreckungstitel vom Feststellungsvermerk verdrängt wird, beruht auf dem insolvenzspezifischen Ordnungszusammenhang und auf Verkehrssicherheitsgründen. ${ }^{855}$ Dadurch, dass sämtliche Vollstreckungstitel zwecks Anmeldung der titulierten Forderungen in das Insolvenzverfahren gezogen (vgl. §§ 174 I 2; 179 II InsO) und neu tituliert werden, liegt ein deutlicher Hinweis dafür, dass der alte Titel - jedoch ohne Beeinträchtigung seiner Rechtskraftwirkung ${ }^{856}$ - als Titel durch einen neuen „verdrängt“ wird. Hierfür spricht insbesondere, dass der frühere Titel hinsichtlich der in der Insolvenz ausgeschütteten Quote stets „überholt“ ist. Die eingetretene Teilbefriedigung entzieht dem früheren Titel zwar nicht per se die Vollstreckbarkeit, jedoch wäre der Schuldner letztlich auf die §§ 766, 767 ZPO angewiesen. Dieser mit dem Insolvenzverfahren typischerweise verbundenen Veränderung der Rechtslage soll daher der Feststellungsvermerk von vornherein Rechnung tragen. Auf dem vollstreckbaren Tabellenauszug sind nämlich die - aus den Insolvenzakten ersichtlich - bereits erfolgten Zahlungen aus der Insolvenzmasse auf die Insolvenzforderung zu vermerken bzw. in Abzug von der festgestellten Forderungshöhe zu bringen (vgl. § 757 ZPO), ${ }^{857}$ da das Nachforderungsrecht des Insolvenzgläubigers gem. § 201 I InsO nur noch so weit besteht, wie seine Forderung noch keine Befriedigung fand. Dem dieser veränderten Rechtslage näherstehende Tabellenvermerk gebührt deshalb der Vorrang für die Nachhaftung, wie es auch die Regelung des § 201 II 1 InsO eindeutig nahe legt. Die Vollstreckbarkeit des früheren Titels wird insoweit verdrängt. ${ }^{858}$ Darüber hinaus stellt die Regelung in § 733 ZPO klar, dass die Rechtsordnung natürlich ein Interesse

855 Hinsichtlich anderweitiger Begründungen siehe zusammenfassend bei Kübler/Prütting/Bork/Holzer, § 201 Rn.17 m.w.N und deren ausführliche Besprechung bei Gaul, in FS/Weber, S.155ff.

856 Für einen vollständigen Rechtskraftverlust des früheren Titels fehlt es an einer eindeutigen gesetzlichen Anordnung. - HHK/Herchen, § 201 Rn.15; vgl. auch ausführlich Gaul, in FS/Weber, S.174f.; Pape, KTS 1992, 185 (189f.); Jaeger/Weber, $\S 164$ Rn.6.

857 Uhlenbruck/Uhlenbruck, § 201 Rn.7; MüKo-ZPO/Heßler, § 757 Rn.42f.; Gaul, in: FS/Weber, S.172; Jaeger/Weber, KO, § 164 Rn.7a m.w.N.

858 Gaul, in: FS/Weber, S.174f., 178; Jaeger/Weber, § 164 Rn.6. 
dahingehend hat, nicht mehrere, auch bei eventuell inhaltlicher Abweichung ( $\S$ 41ff. InsO) letztlich denselben Anspruch betreffende Titel ${ }^{859}$ vollstreckbar nebeneinander wirken zu lassen. ${ }^{860}$ Insoweit bietet die Insolvenzordnung durch die Regelung des $\S 178$ II 3 InsO auch eine entsprechende Verfahrensvorkehrung, um dem Feststellungsvermerk den Vorrang vor dem früheren Titel zu sichern, indem die erfolgte Insolvenzfeststellung vor Erteilung eines vollstreckbaren Tabellenauszugs auf dem früheren Titel vom Urkundsbeamten der Geschäftsstelle zu vermerken ist. ${ }^{861}$ Dieser „vollstreckungsentwertende“ Vermerk lässt bei einem Vollstreckungsversuch aus der ursprünglichen Titelausfertigung ohne weiteres die insolvenzmäßige (Zweit-)Titulierung durch den Feststellungsvermerk für das Vollstreckungsorgan erkennen, das diesen Vermerk von Amts wegen und notfalls auf Erinnerung gem. § 766 ZPO zu beachten hat. ${ }^{862}$

Diese „Verdrängungstheorie“ kann jedoch nur so weit reichen, als der bereits früher titulierte Anspruch des Insolvenzgläubigers nicht auch insgesamt am Insolvenzverfahren teilnehmen kann. So ist der Insolvenzgläubiger mit den laufenden Zinsen seiner angemeldeten Insolvenzforderung seit der Eröffnung des Insolvenzverfahrens grundsätzlich nur nachrangiger Insolvenzgläubiger und nimmt nur nach besonderer Aufforderung zur Anmeldung durch das Insolvenzgericht am Insolvenzverfahren teil ( $§ 174$ III InsO). Wie bereits dargelegt, ${ }^{863}$ wird dies in der Praxis sicherlich die Ausnahme bleiben. Soweit der Insolvenzgläubiger daher Ansprüche, die im früheren Titel mit tituliert sind, zum Insolvenzverfahren gar nicht erst anmelden kann, bildet auch der Tabellenauszug hierüber keinen neuen zur Zwangsvollstreckung geeigneten Titel, so dass eine „Verdrängung“ des früheren Titels insoweit nicht in Betracht kommen kann. Daher bleibt der frühere Titel

859 Auf die auch über das Verfahren hinauswirkende Inhaltsänderung der angemeldeten und festgestellten Insolvenzforderung durch die $\S \S 41 \mathrm{ff}$. InsO soll hier nicht näher eingegangen werden. Siehe dazu die Ausführungen bei MüKo-InsO/Bitter, § 45 Rn.36ff.; Uhlenbruck/Uhlenbruck, § 201 Rn.8.

860 Insofern liegt hier auch keine Rechtskraftkollision i.S.d. § 580 Nr.7 ZPO vor, da die Insolvenzordnung mit Rücksicht auf Ziel und Zweck des Insolvenzverfahrens auch die Anmeldung bereits rechtskräftig titulierter Forderungen vorschreibt (arg. § 179 II InsO) und damit die negative Rechtskraftwirkung gesetzlich suspendiert. Vgl. Gaul, in: FS/Weber, S.172; Jaeger/Weber, KO, § 164 Rn.6. Siehe bereits unter D.IV.4.b) (S.50ff.).

862 Uhlenbruck/Uhlenbruck, § 201 Rn.9; MüKo-InsO/Hintzen, § 201 Rn.38 m.w.N.

863 Siehe bereits unter C.III. (S.20ff.) und E.II.6. (S.203f.). 
insbesondere für nicht anmeldbare nachrangige Insolvenzforderungen im Sinne des § 39 InsO die zur Zwangsvollstreckung geeignete und bestimmte Grundlage. ${ }^{864}$

Klarstellend sei darauf hingewiesen, dass sich die eben gemachten Ausführungen natürlich nur auf solche vorinsolvenzlich titulierten Insolvenzforderungen beziehen, die zum Insolvenzverfahren angemeldet und festgestellt worden sind und die auch vom Schuldner unwidersprochen geblieben sind bzw. bei denen dessen Widerspruch im Feststellungsverfahren beseitigt worden ist (§ 201 II 1, 2 InsO). Ist dies nicht der Fall, weil die titulierten Forderungen gar nicht erst angemeldet oder zwar angemeldet, aber nicht gem. §§ 178 I, III, 201 II 1 InsO vollstreckbar festgestellt werden konnten, bleibt der ursprüngliche Titel nach Wegfall der Vollstreckungssperre ( $\S \S ~ 88,89$ InsO) für die uneingeschränkte Zwangsvollstreckung maßgeblich. ${ }^{865}$ In diesen Fällen kann nämlich kein vollstreckbarer Tabellenauszug vom Urkundsbeamten (§ 724 II ZPO) oder Rechtspfleger (bei titelergänzenden oder titelübertragenden Vollstreckungsklauseln, vgl. § 20 Nr.12 RPflG) erteilt werden, der die Vollstreckbarkeit des früheren Titels verdrängt. Doch sind auch in diesem Fall die Vorschriften der Restschuldbefreiung zu beachten (§ 294 InsO).

Schließt sich daher ein Restschuldbefreiungsverfahren an das Insolvenzverfahren an, wirkt gem. § 294 I InsO nach Sinn und Zweck des Insolvenzverfahrens und nach dem sich hieraus ergebenden insolvenzrechtlichen Gleichbehandlungsgrundsatz auch das Verbot der Zwangsvollstreckung fort, ${ }^{866}$ so dass auch hier eine Tabellenausfertigung erst nach der gerichtlichen Entscheidung über die Erteilung, Versagung oder vorzeitige Beendigung des Restschuldbefreiungsverfahrens zu erfolgen hat. Diese Verfahrensweise ist zwar nicht explizit für das Restschuldbefreiungsverfahren geregelt, ergibt sich aber aus der nach Sinn und Zweck entsprechend anwendbaren Regelung des § 201 II 3 InsO, da auch im Restschuldbefreiungsverfahren erst zum Zeitpunkt der entsprechenden Entscheidung des Insolvenzgerichts die (endgültige) Höhe

\footnotetext{
864 LG Bielefeld DGVZ 1991, 120 (120); HHK/Herchen, § 201 Rn.12; FK/Kießner, § 201 Rn.11b; MüKo-InsO/Hintzen, § 201 Rn.37; Uhlenbruck/Uhlenbruck, § 201 Rn.9; Pape, KTS 1992, 185 (188f.); wohl auch schon Jaeger/Weber, KO, § 164 Rn.6. 
des vollstreckbaren Restbetrags der insoweit unbefriedigt gebliebenen Insolvenzforderung bestimmt werden kann. Dadurch wird eine größtmögliche Rechtssicherheit für den Schuldner geschaffen, der sich je nach Situation und Entscheidung des Insolvenzgerichts über das Verfahren nur mit solchen Titeln aus der Tabelle konfrontiert sehen soll, die entsprechend der Entscheidung über die Restschuldbefreiung auch (noch) ihre Berechtigung gegenüber dem Schuldner aufweisen, so dass eine ansonsten eventuell notwendige Einlegung von Rechtsmitteln des Schuldners (§§ 766, 767 ZPO) gegen vorzeitig erteilte Tabellenausfertigungen unterbunden werden kann. Insolvenzgläubiger, deren Insolvenzforderung einer erteilten Restschuldbefreiung unterfallen - also insbesondere solche Insolvenzforderungen, die nicht mit dem Forderungsattribut angemeldet bzw. nicht zur Tabelle festgestellt oder bei denen ein Widerspruch gegen das Forderungsattribut nicht beseitigt werden konnte (§ 302 Nr.1 InsO) - kann dann schon kein vollstreckbarer Auszug mehr aus der Tabelle erteilt werden.

Aufgrund des eindeutigen Wortlauts des § 302 Nr.1 i.V.m. § 301 I 2 InsO sowie nach Sinn und Zweck der gem. §§ 174ff. InsO zu erfolgenden Anmeldung sämtlicher Insolvenzforderungen zum Insolvenzverfahren - die eben nicht nur die Prüfung von untitulierten, sondern gerade auch von bereits titulierten Insolvenzforderungen hinsichtlich ihrer Berechtigung (vgl. § 189 InsO) ermöglichen soll - werden von der Restschuldbefreiung auch die mit dem Forderungsattribut bereits titulierten Insolvenzforderungen erfasst, die nicht zum Verfahren angemeldet worden sind und daher gerade nicht die Voraussetzungen des § 302 Nr.1 InsO erfüllen. Von der Restschuldbefreiung sind gem. § 302 Nr.1 InsO nämlich nur solche Insolvenzforderungen ausgenommen, die - unabhängig von einer vorherigen Titulierung - zur Tabelle als Insolvenzforderung aus einer vorsätzlich begangenen unerlaubten Handlung angemeldet und - widerspruchsfrei - mit dem Forderungsattribut festgestellt werden konnten. ${ }^{867}$ Wäre dies anders, bestünde für den Schuldner schon von Anbeginn des Insolvenzverfahrens gar nicht die Möglichkeit, seine Chancen auf eine spätere Restschuldbefreiung hinsichtlich unangemeldeter Insolvenzforderungen mit Forderungsattribut richtig einschätzen zu

867 Vgl. FK/Ahrens, § 302 Rn.19. 
können. Dies war aber gerade die Intention des Gesetzgebers bei der Einführung und Ergänzung der §§ 174 II, 175 II, 302 Nr.1 InsO. ${ }^{868}$ Darüber hinaus bestimmt $\S 301$ I 2 InsO, dass sämtliche zum Verfahren unangemeldet gebliebenen Insolvenzforderungen - und das unabhängig davon, ob und wie sie betitelt oder unbetitelt sind - der Restschuldbefreiung unterfallen, was in Korrespondenz mit $\S 302$ Nr.1 InsO eine effektive Restschuldbefreiung des Schuldner sichern soll, indem sich der Insolvenzgläubiger der Restschuldbefreiung nicht dadurch entziehen kann, dass er die Anmeldung seiner Insolvenzforderung unterlässt. Das gilt selbst dann nicht, wenn seine Insolvenzforderung bereits bei Eröffnung des Insolvenzverfahrens als Forderung aus vorsätzlich begangener unerlaubter Handlung betitelt war.

\section{Berichtigung unrichtiger oder fehlender Eintragung in die Tabelle}

Die Rechtskraftwirkung des Feststellungsvermerks gem. § 178 III InsO soll sich, wie es generell bei in materieller Rechtskraft (§ 322 I ZPO) erwachsenen Entscheidungen - etwa in Form der Urteilsformel - der Fall ist, natürlich nur an das tatsächliche Prüfungsergebnis knüpfen. Weder die verfahrensrechtlichen Vorschriften der Insolvenzordnung noch die über § 4 InsO anzuwendende Zivilprozessordnung enthalten jedoch eindeutige Regelungen darüber, ob und wie die Insolvenztabelle berichtigt oder ergänzt werden kann. Aufgrund der Rechtskraftwirkung durch § 178 III InsO besteht bei Unrichtigkeiten der Tabelle allerdings ein „unabwendbares Bedürfnis“, die Feststellungsvermerke und auch die Tabelle allgemein, die nicht das tatsächliche Ergebnis des Prüfungstermins wiedergibt, ${ }^{869}$ von Amts wegen oder auf Antrag eines Verfahrensbeteiligten berichtigen zu können. ${ }^{870}$ Die Eintragung eines Widerspruchs könnte zu Unrecht erfolgt oder nicht erfolgt sein; es könnte

${ }^{868}$ Siehe bereits unter E.I.4.f)bb)(2) (S.175ff.).

869 Für die Berichtigung unrichtiger Eintragungen sind alle Abweichungen vom Willen bei der Eintragung, also die Abweichung zwischen Willensbildung und Willenserklärung, entscheidend. AG Köln NZI 2005, 171 (171), Hess, InsO, § 178 Rn.58.

870 Allgemeine Meinung: BGHZ 91, 198 (201); OLG Celle KTS 1964, 118 (118f.); MüKo-InsO/Schumacher, \& 178 Rn.51; Jaeger/Weber, KO, § 145 Rn.3b; Uhlenbruck/Uhlenbruck, § 178 Rn.21 m.w.N. 
aber auch ein Tabelleneintrag ganz fehlen. Die

Berichtigungsmöglichkeiten

der

§§ 319, 320 ZPO können im Rahmen der Insolvenztabelle jedoch keine Anwendung finden. § 319 ZPO setzt eine Entscheidung des Gerichts voraus, während das Insolvenzgericht zur Tabelleneintragung lediglich Erklärungen des Insolvenzverwalters, der Insolvenzgläubiger und des Schuldners entgegenzunehmen und zu beurkunden hat, ohne hierzu eine Entscheidung zu treffen. ${ }^{871} \S 320 \mathrm{ZPO}$ ist nicht entsprechend anwendbar, da nicht der einem Urteil zugrunde liegende Tatbestand, sondern das Ergebnis der Prüfung eines durch Anmeldung festgelegten „Tatbestandes“ ${ }^{\star 872}$ berichtigt werden soll, das nach $\S 178$ I, III InsO i.V.m. $\S 322$ I ZPO der Wirkung des Tenors eines Urteils entspricht. ${ }^{873}$ Die Tabelle und mit ihr der Feststellungsvermerk ist vielmehr Teil des zu führenden Protokolls über den Prüfungstermin, ${ }^{874}$ so dass $\S 164$ I ZPO i.V.m. § 4 InsO entsprechend Anwendung findet. ${ }^{875}$

Berichtigungen sind auch noch nach Beendigung des Insolvenzverfahrens zulässig. ${ }^{876}$ Dies folgt ohne weiteres daraus, dass die Tabelle aufgrund erst nach der Schlussverteilung in Feststellungsprozessen ergehende Entscheidungen zu berichtigen ist (§§ 189 II, 198, 203 I Nr.1, 183 II InsO). Aber auch im Übrigen besteht nicht zuletzt im Hinblick auf die Vollstreckungswirkung des Tabelleneintrags ein ausreichendes Bedürfnis für eine Tabellenberichtigung nach Abschluss des Verfahrens (§§ 201, 202 InsO).

Unverständlich im Zusammenhang mit der Berichtigungsmöglichkeit falscher Eintragungen ist jedoch die vielfach zu lesende, aber ohne nähere

871 Der § 319 ZPO soll zwar als allgemeiner Rechtsgedanke auch im Rahmen anderer Verfahrensordnungen Anwendung finden (vgl. BGHZ 106, 370 (372); MüKoZPO/Musielak, § 319 Rn.2); dies kann jedoch nur soweit gelten, als dass eine Entscheidung durch das Gericht getroffen wird, woran es im Zusammenhang mit der freiwilligen Feststellung im Insolvenzverfahren jedoch gänzlich fehlt. Siehe auch Darstellung bei Jaeger/Weber, KO, § 145 Rn.3b.

872 Grund und Betrag der Insolvenzforderung.

873 Hinzu kommt, dass $\S 320$ ZPO einen besonderen Termin vorsieht (§ 320 III ZPO) und die Anwendung bei einem Richterwechsel versagt (§ 320 IV ZPO).

874 So schon KO-Motive, S.363 = Hahn, KO, S.326.

875 MüKo-InsO/Schumacher, § 178 Rn.51; Nerlich/Römermann/Becker, § 178 Rn.17. Nach OLG Celle MDR 1965, 65 (65) findet § 164 III 2 ZPO - Berichtigung nur durch dieselbe Urkundsperson - bei der Tabellenberichtigung jedoch keine Anwendung.

876 BGHZ 91, 198 (201); OLG Celle MDR 1964, 65 (65); OLG Hamm Rpfleger 1965, 78 (78); Uhlenbruck/Uhlenbruck, § 178 Rn.24; Schumacher, § 178 Rn.52 m.w.N.; a.A. RGZ 22, 153 (155). 
Begründung geäußerte Aussage, ${ }^{877}$ dass sich die Rechtskraftwirkung wohl ausschließlich an das richtige Prüfungsergebnis knüpfen würde, also ein Feststellungsvermerk falschen Inhalts gar nicht erst in Rechtskraft erwachsen könnte. Dem kann nämlich aufgrund des eindeutigen, hinsichtlich der Rechtskraftwirkung zwischen „richtiger“ und „falscher“ Beurkundung undifferenzierenden Wortlauts des § 178 III InsO nicht gefolgt werden. ${ }^{878}$ Auch gerichtliche Entscheidungen etwa, deren Urteile den tatsächlichen Inhalt des Verfahrens nicht richtig wiedergeben, erwachsen grundsätzlich in Rechtskraft, um zu Rechtssicherheit und Rechtsfrieden beizutragen. ${ }^{879}$ Dieser Wirkung steht $\S 178$ III InsO durch seinen Wortlaut in nichts nach. ${ }^{800}$ Und gerade diese grundsätzliche Rechtskraftwirkung des Feststellungsvermerks begründet doch schließlich auch das „unabwendbare Bedürfnis“ der Berichtigungsmöglichkeit der Tabelle.

Hiervon klar zu trennen ist der bereits dargestellte ${ }^{881}$ aber auch ganz andere Fall, dass eine Nicht-Insolvenzforderung, also eine Forderung die nicht der materiellrechtlichen Definition der §§ 38, 39 InsO entspricht, angemeldet und festgestellt wird. Deren Feststellungsvermerk kann - aber aufgrund der eindeutigen gesetzlich festgelegten Verfahrenssystematik der §§ $174 \mathrm{ff}$. i.V.m. §§ 38, 39 InsO - tatsächlich nicht in Rechtskraft erwachsen. Gleichwohl sind auch solche Eintragungen, um Missverständnisse hinsichtlich der Aussagekraft der Tabelle vorzubeugen, von Amts wegen und/oder auf Antrag zu berichtigen.

Als Rechtsmittel gegen die eine Berichtigung anordnende oder ablehnende Entscheidung des Rechtspflegers ist die sofortige Erinnerung (§ 11 II RPflG) zulässig; gegen die Entscheidung des Abteilungsrichters ist hingegen kein weiteres Rechtsmittel gegeben (§§ 6 I InsO, 11 II RPflG).

877 Siehe etwa MüKo-InsO/Schumacher, § 178 Rn.51; Küber/Prütting/Pape, § 178 Rn.14; Uhlenbruck/Uhlenbruck, § 178 Rn.21f. jeweils m.w.N., die wohl allgemein auf eine nicht eindeutige Formulierung des OLG Hamm Rpfleger 1965, 78 (78f.) abstellen. Die übrigen zur Begründung angeführten Entscheidungen (z.B. BGHZ 91, 198ff. oder OLG Nürnberg JW 1931, 2158f.) beziehen sich nämlich nur auf die grundsätzlich notwendige Berichtigungsmöglichkeit von falschen Feststellungsvermerken. Sie enthalten hingegen keinerlei Aussage hinsichtlich der angeblich grundsätzlich fehlenden Rechtskraft eines falschen Feststellungsvermerks. Vgl. auch Nerlich/Römermann/Becker, § 178 Rn.17; äußerst kritisch auch Gaul, FS/Weber, S.174f. mit Fn.90. 


\section{Rechtsbehelf gegen die festgestellte Insolvenzforderung}

Von der (bloßen) Korrekturmöglichkeit offensichtlich unrichtiger Eintragungen in die Tabelle, die von Amts wegen oder auf Antrag berichtigt werden können, ${ }^{882}$ sind die Rechtsbehelfsmöglichkeiten gegen die ordnungsgemäßen, also den Verlauf des Prüfungstermins richtig wiedergebenden Eintragungen in die Tabelle, zu unterscheiden. Den Widerspruchsberechtigten stehen gegen die fehlerfreie Eintragung festgestellter Insolvenzforderungen in die Tabelle und deren Rechtskraftwirkung nur noch diejenigen Rechtsbehelfe zur Verfügung, die gegen ein rechtskräftiges Urteil gegeben wären (vgl. § $178 \mathrm{III}$ InsO) ${ }^{883}$ Als Rechtsbehelfe gegen nachträgliche Einwendungen gegen die rechtskräftige Feststellung der Insolvenzforderung kommen dabei z.B. in Frage: die

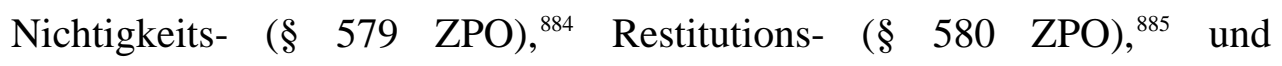
Vollstreckungsgegenklage (§ $767 \mathrm{ZPO})^{886}$ sowie die Klage aus § 826 BGB, wenn der Insolvenzgläubiger die Feststellung sittenwidrig herbeigeführt hat oder die Feststellungswirkung in sittenwidriger Weise ausnutzt. ${ }^{887}$ Weiterhin können Inhalt und Tragweite der Eintragung einer festgestellten Insolvenzforderung mit Hilfe einer Feststellungsklage (§ 256 ZPO) geklärt werden. ${ }^{888}$

Die Wahrnehmung des Schuldnerinteresses daran, dass nur berechtigte Insolvenzgläubiger an der Verteilung der Insolvenzmasse teilnehmen, ist während des Insolvenzverfahrens ausschließlich dem Verwalter zugewiesen. $^{889}$ Der Schuldner selbst kann daher durch einen der vorgenannten Rechtsbehelfe die Teilnahme des Insolvenzgläubigers an den Verteilungen nicht hindern. Nach Beendigung des Verfahrens hingegen stehen auch ihm sämtliche Rechtsbehelfe gegen die Vollstreckung aus dem Tabelleneintrag zur Verfügung. ${ }^{890}$

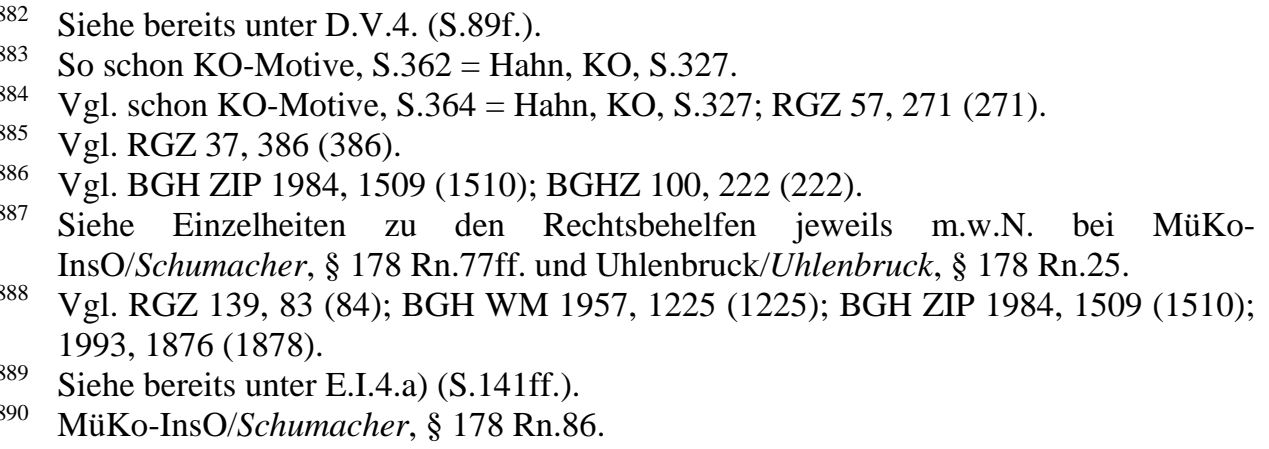




\section{Wirkung der Nicht-/Feststellung auf die Rechte des Anmeldenden}

Durch die Feststellung der angemeldeten Insolvenzforderung sichert sich der Insolvenzgläubiger die uneingeschränkte Verfahrensteilnahme und die damit verbundenen Verfahrensrechte. Dies gilt sowohl für sein Widerspruchsrecht im Prüfungstermin ( 176 InsO) bzw. im schriftlichen Verfahren (vgl. §§ 177 I 2; 5 II 1 InsO) und sein Stimmrecht (§ 77 I InsO) in sämtlichen Gläubigerversammlungen als auch hinsichtlich des Rechts auf Befriedigung seiner angemeldete Insolvenzforderung durch Teilnahme am Verteilungsverfahren ( $\S 187$ I, 189 I InsO) und an entsprechender Ausschüttung der Quote. Letzteres gilt jedoch nur insoweit, als der Insolvenzgläubiger seine Forderung so frühzeitig angemeldet hat, dass sie noch vor Niederlegung des Schlussverzeichnisses zur Prüfung gestellt und entsprechend im Verzeichnis Berücksichtigung finden konnte (vgl. § 189 I InsO). ${ }^{891}$ Darüber hinaus nimmt der Insolvenzgläubiger mit seinen festgestellten Forderungen - im Fall ihrer Aufnahme ins Schlussverzeichnis - auch bei Verteilungen während der Wohlverhaltensphase bei beantragter Restschuldbefreiung teil ( $\S \S 287$ II, 292 I InsO). Nach Aufhebung des Insolvenzverfahrens kann der Insolvenzgläubiger zudem eine vollstreckbare Ausfertigung aus der Tabelle in Höhe des unbefriedigten Restbetrags der Insolvenzforderung beantragen (§ 201 II 3 InsO). Schließt sich an das Insolvenzverfahren ein Restschuldbefreiungsverfahren an, so darf eine vollstreckbare Tabellenausfertigung entsprechend § 201 II 3 InsO erst nach der Entscheidung des Insolvenzgerichts über die Erteilung/ Versagung der Restschuldbefreiung oder nach einer vorzeitigen Beendigung des Restschuldbefreiungsverfahrens erfolgen und nur unter Berücksichtigung der entsprechend an die Entscheidung geknüpften Rechtsfolgen. ${ }^{892}$

Wird die Forderung eines Insolvenzgläubigers aufgrund eines Widerspruchs hingegen nicht zur Tabelle festgestellt, verliert der Insolvenzgläubiger seine formelle Insolvenzgläubigerstellung und damit sämtliche Verfahrensrechte nur dann, wenn der Widerspruch gegen seine angemeldete Forderung - je nach Betreibungslast $\quad$ im Feststellungsprozess gem. $\S \S 179 \mathrm{ff}$. InsO weiterverfolgt wird und seine

891 Siehe bereits unter E.II.3.b) (S.194ff.).

892 Siehe bereits unter E.III.5.c) (S.225ff.). 
Forderung im Ergebnis nicht als Insolvenzforderung bestimmten Rangs und Betrags festgestellt werden kann. Aufgrund der so festgestellten gänzlich fehlenden Insolvenzgläubigerstellung gem. § 38 InsO ist der betroffene „Gläubiger“ folglich nicht einmal mehr Verfahrensbetroffener. Der Widerspruch des Schuldners hat - anders in der Eigenverwaltung keine Auswirkungen auf die Verfahrensrechte des Insolvenzgläubigers im Insolvenzverfahren, sondern hindert nur die nachinsolvenzliche Vollstreckung aus der Tabelle. 


\section{Conclusio}

Übergreifend lässt sich hinsichtlich der Anmeldung, Prüfung und Feststellung von Insolvenzforderungen im Verfahren der §§ 174ff. InsO festhalten, dass die mögliche Begründung und der grundsätzliche Verlust sämtlicher Verfahrensrechte innerhalb dieses Verfahrens ausschließlich an die formelle Insolvenzgläubigerstellung gebunden ist. Die formelle Insolvenzgläubigerstellung kann nur durch die gem. § 174 InsO formell ordnungsgemäße Anmeldung von Insolvenzforderungen ( $\S \S 38,39$ InsO) beim Insolvenzverwalter erlangt werden, die noch im Schlusstermin erfolgen kann. Alle angebrachten Anmeldungen werden vorab grundsätzlich nur vom Insolvenzverwalter auf ihre formelle Zulässigkeit zum Insolvenzverfahren geprüft. Über die weitere materiellrechtliche Teilnahmeberechtigung der einzelnen angemeldeten Forderungen der Insolvenzgläubiger im Insolvenzverfahren entscheiden ausschließlich die formellen Insolvenzgläubiger und der Insolvenzverwalter durch Ausübung ihres feststellungshindernden Widerspruchsrechts im Prüfungstermin/ Schlusstermin oder im schriftlichen Prüfungsverfahren, an deren Ergebnisse die jeweils entsprechenden Stimmrechtsfeststellungen der Insolvenzgläubiger gem. § $77 \mathrm{InsO}$ zu knüpfen sind. Der Schuldner hingegen hat - außer im Sonderfall der Eigenverwaltung - durch seinen lediglich vollstreckungshindernden Widerspruch nur Einfluss auf seine persönliche Nachhaftung, nicht aber auf das Insolvenzverfahren und damit auf die Verfahrensrechte der Insolvenzgläubiger selbst. Daher kann auch nur der Schuldner oder ein entsprechend konkurrierender Insolvenzgläubiger, nicht aber der Insolvenzverwalter - dessen Gesamtinteressenvertretung sich insofern auf das Insolvenzverfahren beschränkt - dem Forderungsgrund der vorsätzlich begangenen unerlaubten Handlung einer angemeldeten Insolvenzforderung hinsichtlich der Nachhaftung widersprechen. Die Erörterungen hinsichtlich der Verfolgung von Widersprüchen insbesondere gegen Insolvenzforderungen aus vorsätzlich begangener unerlaubter Handlung haben dabei gezeigt, dass mit den derzeitigen insolvenzverfahrensrechtlichen Regelungen - insbesondere des § 184 I InsO - das mit den §§ 174 II, 175 II, 302 Nr.1 InsO verfolgte gesetzgeberische Ziel, dem Schuldner frühzeitig Gewissheit 
über den Umfang seiner Nachhaftung und damit über seinen Antrag auf Restschuldbefreiung zu verschaffen, aufgrund der gleichermaßen zu berücksichtigenden Insolvenzgläubigerinteressen nicht erreicht wird. Daher kann es nur Aufgabe des Gesetzgebers sein, unter Berücksichtigung der aufgezeigten unterschiedlichen Interessen für die notwendige Rechtssicherheit aller Verfahrensteilnehmer, insbesondere aber des Schuldners, zu sorgen. Die Einführung einer oder weiterer Ausschlussfrist(en) ist durch die aufgezeigte eindeutige Differenzierungsmöglichkeit bei der Weiterverfolgung von Widersprüchen nach Qualität der Insolvenzforderung und der damit verbundenen Betreibungslast, dem Zeitpunkt ihrer ordnungsgemäßen Anmeldung und Prüfung in Bezug zum Verfahrensstand und zu den jeweils entsprechenden (Feststellungs-) Interessen des Schuldners und des Insolvenzgläubigers umfassend und interessengerecht möglich und auch handhabbar, wie es der Gesetzgeber unter Kenntnis dieser Problematik schließlich in $\S 184$ II InsO bereits getan hat. Gleichzeitig würden durch die Einführung entsprechender Regelung(en) in Anlehnung an den Regelungsinhalt des § 184 II InsO willkürlichen Widersprüchen - insbesondere gegen titulierte Insolvenzforderungen, die durch den Widersprechenden doch nicht weiterverfolgt werden - vorgebeugt werden. Das würde nicht nur eine Entlastung der Gerichte durch unnötige insolvenzrechtliche Feststellungsklagen seitens der Widerspruchsbetroffenen bedeuten, sondern auch schneller Klarheit über die Verfahrensrechte der einzelnen Insolvenzgläubiger mit sich bringt. Dies gilt nicht nur hinsichtlich eines positiven, sondern gerade auch hinsichtlich eines negativen Ausgangs des Feststellungsverfahrens der angemeldeten Forderungen, da der Verlust der formellen Insolvenzgläubigerstellung und der damit einhergehende Verlust der Verfahrensrechte und die weitere Einwirkungsmöglichkeit des Anmeldenden auf das Insolvenzverfahren nur durch eine endgültige (negative) Feststellungsentscheidung des Prozessgerichts über die angemeldete Forderung als Insolvenzforderung erfolgen kann. Daher ist die umfassende Regelung von Ausschlussfristen in den $\S \S 179,184$ InsO für die Erhebung von insolvenzrechtlichen Feststellungsklagen seitens des Gesetzgebers nicht nur hinsichtlich des Schuldnerantrags auf 
Restschuldbefreiung, sondern generell für die Interessen aller Verfahrensteilnehmer angebracht und wünschenswert. 


\section{Curriculum Vitae}

Name Mark A. Jaeschke

Geburtstag $\quad 25.10 .1979$ in Hannover

Schulbildung

1986 - 1990 Grundschule Kurt-Schumacher-Schule Anderten, Hannover

1990 - 1991 Orientierungsstufe Anderten, Hannover

1991 - 1992 Orientierungsstufe Mellendorf, Wedemark

1992 - 1999 Gymnasium Mellendorf, Wedemark

Wehrdienst

1999 - 2001

Panzergrenadierbataillon 72, Hamburg-Fischbek

\section{Studium}

WS 2001/02

Studium der Rechtswissenschaften an der Philipps-Universität Marburg WS 2003/04 Studium der Rechtswissenschaften an der Georg-August-Universität Göttingen

Oktober 2006 Erstes Juristisches Staatsexamen

\section{Promotion}

Ab 2007

Doktorand bei Professor Dr. Martin Ahrens, Lehrstuhl für Bürgerliches Recht, Anwaltsrecht und Zivilprozessrecht an der Georg-August-Universität Göttingen

\section{Referendariat}

2008 - $2010 \quad$ Juristischer Vorbereitungsdienst im Oberlandesgerichtsbezirk Celle

Dezember 2010 Zweites Juristisches Staatsexamen

\section{Berufliche Tätigkeit}

Seit März 2011 Rechtsanwalt (Rechtsanwaltskammer Celle) 